\title{
Organocatalyzed Mannich Reactions on Minocycline: Towards Novel Tetracycline Antibiotics
}

\author{
Tirivashe E. Chiwunze ${ }^{a}$, Rafiatu Azumah ${ }^{a}$, Melissa Ramtahal ${ }^{a}$, Anou M. Somboro ${ }^{a}$, \\ Per I. Arvidsson ${ }^{\mathrm{b}}$, Hendrik G. Kruger ${ }^{\mathrm{a}}$, Thavendran Govender ${ }^{\mathrm{a}, \star}$ and Tricia Naicker ${ }^{\mathrm{a}, \mathrm{k}}$ \\ ${ }^{a}$ School of Pharmacy and Pharmacology, University of KwaZulu-Natal, Westville Campus, Durban 4000, South Africa. \\ ${ }^{b}$ Science for Life Laboratory, Drug Discovery and Development Platform, and Division of Translational Medicine and \\ Chemical Biology, Department of Medical Biochemistry and Biophysics, Karolinska Institutet, Stockholm, Sweden.
}

Received 22 January 2016, revised 3 March 2016, accepted 6 March 2016.

\begin{abstract}
Herein, we report the development of a mild synthetic route towards novel minocycline derivatives using the proline-catalyzed three-component Mannich reaction. The reaction conditions were optimized and was then screened for its tolerance to the other popular organocatalysts as well as variation of the ketone and aldehyde substrates. The Mannich adducts were evaluated for their in vitro antibacterial efficacies against Gram-negative and Gram-positive bacteria.
\end{abstract}

KEYWORDS

Minocycline,tetracycline, antibiotics, Mannich reaction, organocatalysis.

\section{Introduction}

The first class of tetracyclines was introduced in the 1950s as safe and effective broad-spectrum antibiotics. These agents have been important medical products for the last 50 years, ${ }^{1}$ however, the misuse of antibiotics has accelerated the natural inevitable phenomenon of resistant strain evolution. Tetracyclines inhibit bacterial growth by interfering with protein synthesis. They attach themselves to the bacterial 30S subunit of the rRNA (A site) which results in the prevention of the binding of aminoacyl-tRNA. ${ }^{2}$ The emergence of resistant bacteria to many of the current antibiotics is a major worldwide threat to public health.

There is an urgent need for the identification and development of novel antibiotics with new modes of action and significant therapeutic efficacies against these pathogens. ${ }^{3}$ Modification of existing antibiotic classes to new derivatives is an option to overcome this rapid drug resistance development. The urgent need to revive the tetracycline has brought about renewed interest to this class of compounds.

Most of the early tetracyclines were naturally occurring molecules (first generation), e.g. from Streptomyces aureofaciens and Streptomyces rimosus, or a product of semi-sythethic methods (second generation), e.g. minocycline and doxycycline (Fig. 1). Despite the great success of the early tetracyclines, improvements had to be made to better the pharmacokinetic properties, antimicrobial potency and also to decrease toxicity. This prompted the development of the third generation,which has led to many more derivatives and to the discovery of tigecycline, used in the fight against resistant strains. ${ }^{4}$ Although tigecycline is the first tetracycline drug to be brought to the market in the last half century, two more tetracyclines (omadacycline and eravacycline) are following in phase III clinical trials.

Structure-activity relationship (SAR) studies $^{6}$ on the tetracycline backbone have shown that it can tolerate structural modifications on positions C4 to C9 (Fig. 1). ${ }^{7}$ These chemical modifications often result in the change of chemical-physical properties and improved ability to overcome drug efflux. Recently K.C. Nicolaou et al.

* To whom correspondence should be addressed. E-mail: naickert1@ukzn.ac.za / govenderthav@icloud.com
(2014), Cuixiang Sun et al. (2015) and Hrvoje Petković et al. (2015) reported novel tetracycline derivatives with extensions on the C and D rings. ${ }^{8}$ These compounds showed antibacterial activity against a wide range of clinically important bacterial isolates, including multidrug-resistant, Gram-negative pathogens. ${ }^{8 a, 8 b}$

Given the recent need and synthetic reports on the tetracycline backbone to yield more potent derivatives such as tigecycline and omadacycline, we envisioned an easy entry to new derivatives based on the commercially available minocycline scaffold through milder synthetic routes.

Biocatalysts and metal complexes are amongst the most popular ways to synthesize optically pure molecules. Asymmetric organocatalysis was developed, in the past decade, as the need for alternatives that are less costly, use of less toxic reagents and more environmental friendly conditions. ${ }^{9}$ The last few years have witnessed a spectacular advancement in this field for the synthesis of medicinally important chiral. ${ }^{9}$ A substantial number of reactions using organocatalysis have been developed that were equivalent or better than conventional synthetic methods. Amongst the approaches that benefited are the well-known Aldol $^{10}$, Mannich ${ }^{11}$ and Michael ${ }^{12}$ reactions, which are powerful strategies in synthetic organic chemistry, as they allow the formation of new C-C bonds. ${ }^{13}$ Recently there is a steady increase in the number of reports that are applying organocatalysis in the field of medicinal chemistry due to its mild and relatively benign approach to asymmetric synthesis. ${ }^{14}$

We have recently reported on the application of organocatalysis in the synthesis of novel $\beta$-lactam derivatives. ${ }^{13 b, 15}$ Herein we report the first organocatalyzed Mannich reactions, to make the D-ring substituent modification, on the minocycline scaffold to increase substituent bulk that can mimic the steric hindrance achieved by tigecycline. The end products of the Mannich reactions are also known as beta-amino ketones. ${ }^{16}$ These compounds have shown the ability to increase the hydrophilic properties of drugs through the introduction of a polar function in their structure. ${ }^{17}$ In other studies, they have been shown to act as prodrugs, releasing the active substance under controlled hydrolytic conditions via deaminomethylation ${ }^{18}$ or 

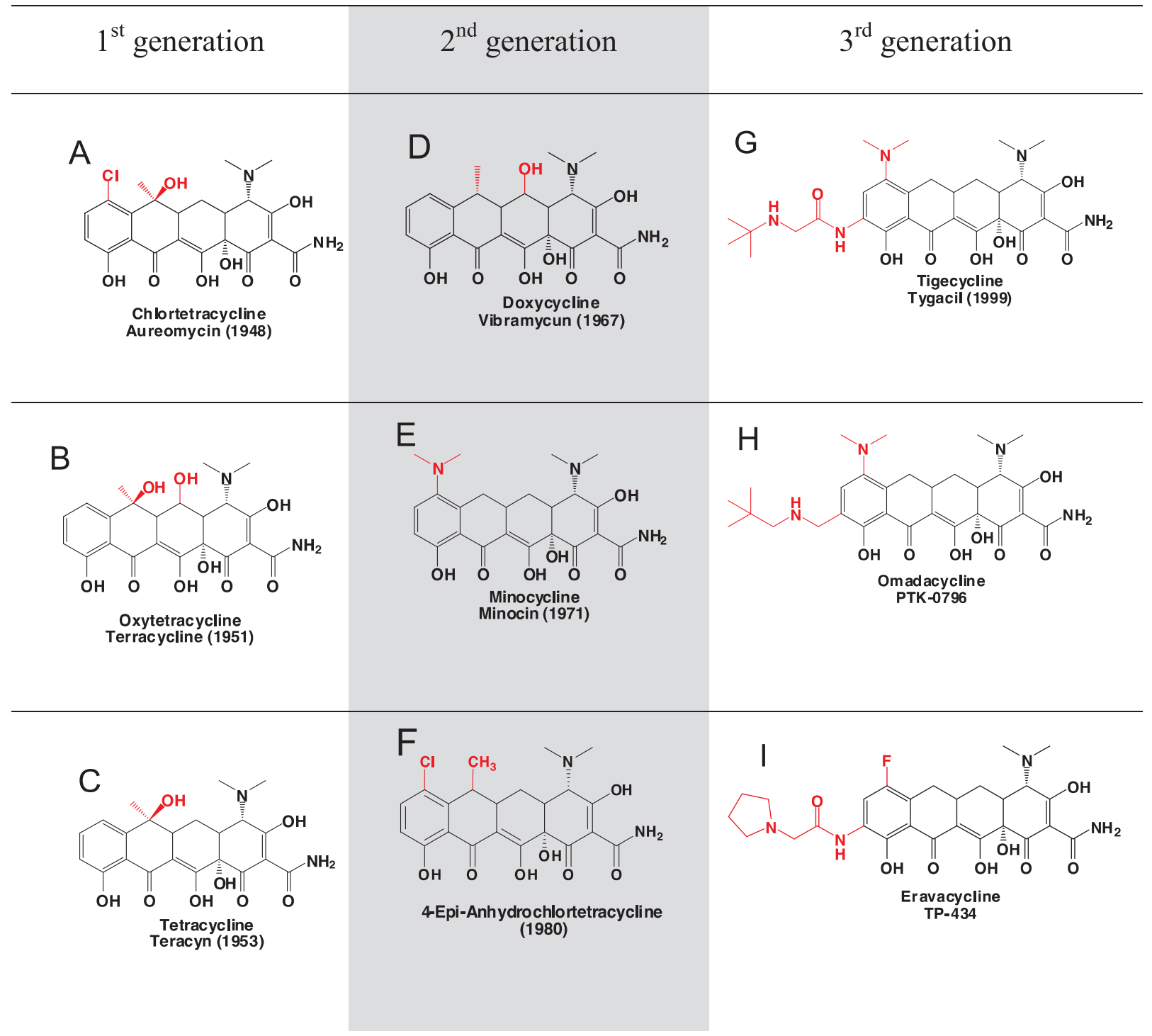

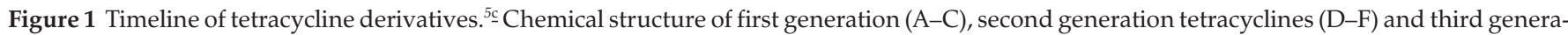
tion (G-I). The numbers in the brackets show the year the drug was discovered.

deamination. ${ }^{19}$ In addition to the organocatalyzed synthesis of new minocycline derivatives, preliminary biological evaluation of these compounds is also outlined.

\section{Results and Discussion}

We successfully carried out a proline catalyzed three component Mannich reaction on the commercially available 9-amino minocycline core witrious aldehydes and ketones (Scheme 1).

The reaction was optimized using a 9-amino minocycline (1), hydroxyl acetone (2) and nitro-benzaldehyde (3) in a one-pot reaction (Table 1 ). Proline was initially chosen to optimize the reaction as it has been well established to catalyze similar transformations. ${ }^{13 a, 20}$ Solvent screening began with dimethylsulphoxide (DMSO) since it is the most commonly reported solvent for Mannich reactions. However, in this case it gave poor conversion $(41 \%)$, which was probably due to the partial solubility of $\mathbf{1}$. Other polar solvents such as ethanol, methanol, 2-isopropanol and dimethylformamide (DMF) in which the amine $\mathbf{1}$ was more soluble was attempted. DMF gave poor conversion (57 \%) despite being able to fully solubilize 1 . The alcoholic solvents exhibited much improved results in the range of 76-96\%, with methanol as the best solvent (96\% conversion) under room<smiles>[R7]CC(=O)C[18CH]C[18O]</smiles><smiles>[R]CC(=O)C([R2])[C@H]([R5])Nc1cc(N(C)C)c2c(c1O)C(=O)C1=C(O)[C@]3(O)C(=O)C(C(N)=O)=C(O)[C@@H](N(C)C)[C@@H]3C[C@H]1C[C@H]2C</smiles>

Scheme 1

Synthesis of tetracycline derivatives via a three-component Mannich reaction. 
Table 1 Optimization of the catalytic asymmetric three-component Mannich reaction.

\begin{tabular}{lllcc}
\hline Entry & Solvent & Catalyst & Mole \% & Conversion \% \\
\hline $\mathbf{1}$ & DMSO & - & - & Trace \\
$\mathbf{2}$ & DMSO & L-proline & 30 & 41 \\
$\mathbf{3}$ & DMF & L-proline & 30 & 57 \\
$\mathbf{4}$ & 2-Isopropanol & L-proline & 30 & 76 \\
$\mathbf{5}$ & Ethanol & L-proline & 30 & 82 \\
$\mathbf{6}$ & Methanol & L-proline & 30 & 96 \\
$\mathbf{7}$ & Methanol & Tetrazol & 30 & 96 \\
$\mathbf{8}$ & Methanol & Jørgensen & 30 & 94 \\
$\mathbf{9}$ & Methanol & Pyrrolidine & 30 & 84 \\
$\mathbf{1 0}$ & Methanol & D-proline & 30 & 95 \\
$\mathbf{1 1}$ & Methanol & L-proline & 20 & 94 \\
$\mathbf{1 2}$ & Methanol & L-proline & 10 & 90 \\
\hline
\end{tabular}

temperature conditions and 8 hours of reaction time.

In order to examine further catalyst effects, we selected a few popular amine catalysts (proline, tetrazol, pyrrolidine and Jørgensen catalyst) that are known to facilitate the progression of Mannich reactions ${ }^{21}$. All of the catalysts showed good activities with conversions in the range of 84-96\%; in alignment with past reports proline typically gave superior results. ${ }^{11 a, 20,22}$ The amount of proline could also be effectively reduced to $10 \mathrm{~mol} \%$ while still obtaining good product conversions ( $>90 \%)$ in a reasonable reaction time $(<24 \mathrm{~h})$.

As previously shown by B. List et al. $(2001)^{6 a}$, that a variety of ketones can be used in such proline-catalyzed Mannich reactions. Reacting four different ketones (butanone, methoxyacetone, ethyl methyl ketone, acetone and hydroxyacetone) with 9-amino minocycline and benzaldehyde furnished the desired products with high conversions $80-98 \%$ (Fig. 2). The aliphatic $(\mathbf{1 a}-\mathbf{1 c})$ and cyclic ketones (1d) generated the desired products while aromatic ketones only produced trace amounts on the HPLC-MS

We next performed an aldehyde screen for the three-component Mannich reactions (Fig. 3). Among the aldehydes screened the $\alpha$-branched aromatic aldehydes (2a-2h) provided the Mannich products in reasonable yields. Not only benzaldehyde derivatives but also heteroaromatic aldehydes (2f, $\mathbf{2 g}$ and $\mathbf{2 h}$ ) worked well. Typically $\alpha$-branched aldehydes are more efficient substrates than the $\alpha$-unbranched aldehydes ${ }^{11 a, 23}$ but in our case; $\alpha$-unbranched aldehydes ( $2 \mathbf{i}$ and $\mathbf{2 j}$ ) only afforded trace amounts of the corresponding Mannich adducts.

The proton and especially the ${ }^{13} \mathrm{C}$ NMR data of the starting material and related products appeared to be problematic. Upon a literature search it was found that the majority of reports did not provide ${ }^{13} \mathrm{C}$ spectral data. ${ }^{8 b, 24}$ It appears that specialized automated triple broadband (ATB) probes are required for the generation of quality ${ }^{13} \mathrm{C}$ spectra. ${ }^{8 c, 25}$

Similarly, the proton spectra were broad and it was difficult to deduce much about the diastereomeric details of the products. Based on the proton spectra as well as HPLC results, it appears that the products may be formed largely as one diastereomer.

The activities of the above new tetracycline derivatives were determined by the agar dilution method following the recommendations of the National Committee for Clinical Laboratory Standards ${ }^{26}$. The analogues tested exhibited antibacterial efficacies against both Gram-positive and Gram-negative bacteria (Table 2).

\subsection{Antibacterial Activity}

Tigecycline and Doxycycline were used as control drugs and their MIC values were in agreement with the Clinical Laboratory Standards Institute (CLSI). ${ }^{40}$ The Mannich adducts in general showed good activities against Gram-positive bacteria, with the best giving a MIC of $1.0 \mu \mathrm{g} \mathrm{mL} \mathrm{m}^{-1}$ against $B$. subtilis. However, these compounds were always less active against Gram-negative bacteria; none of them showed activity against P. aeruginosa and the MICs were $>16 \mu \mathrm{g} \mathrm{mL}^{-1}$ against E.coli with only a couple of exceptions. The poor activities of these compounds against these strains could be as a result of low permeationof the Gramnegative cell wall.

Compounds $\mathbf{1 a}, \mathbf{1 b}, \mathbf{1 c}$ and $\mathbf{1 d}$ showed the effect of changing the ketone on the adducts, with the most polar exhibitiing the better activity. The aldehydes were then varied and the benzaldehyde derivatives demonstrated a mentionable structural-activity interrelation. Addition of a substitution group onto the benzene ring proved to be crucial for the antibacterial activity. Compounds $\mathbf{2 a}, \mathbf{2} \mathbf{b}$ and $\mathbf{2 d}$ have electron-withdrawing substituents on the para position of the benzene ring and exhibited significant improvements compared to the non-substituted

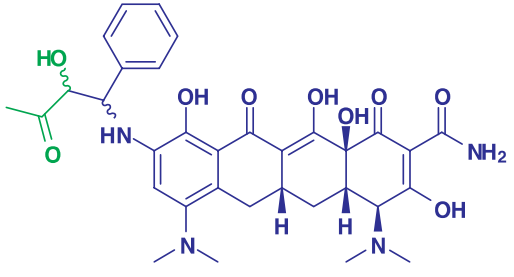

$1 \mathrm{a}, 97 \%, 30 \%$

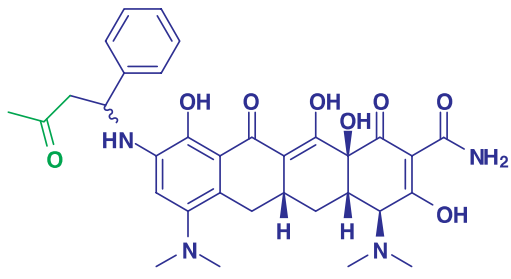

$1 \mathrm{c}, \mathbf{8 0 \%}, \mathbf{3 5 \%}$

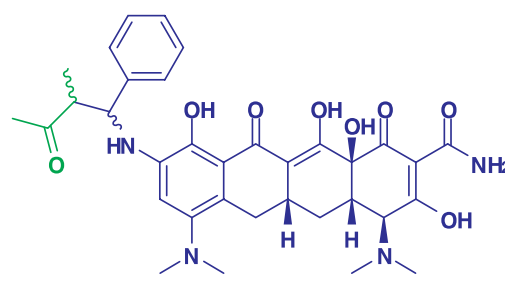

$1 b, 96 \%, 64 \%$

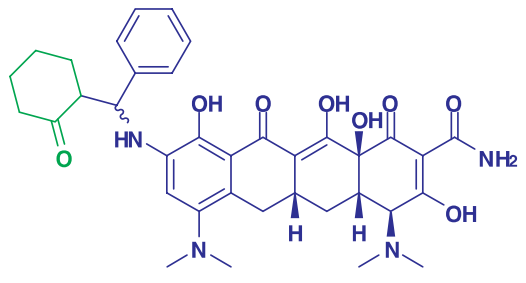

1d, $98 \%, 30 \%$

Figure 2 Three-component Mannich reaction with various ketones. The compound number is followed by the conversion and isolated yield. 


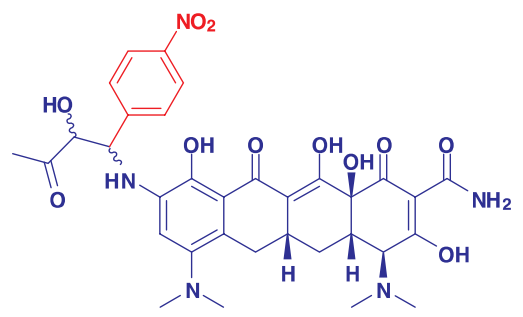

$2 a, 94 \%, 33 \%$

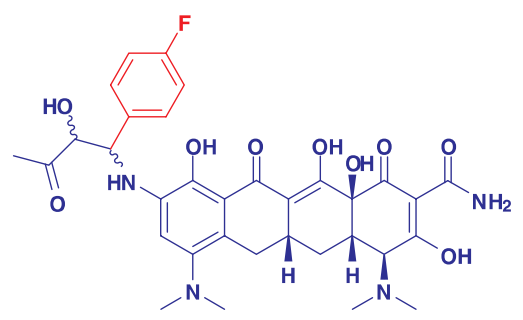

$2 \mathrm{~d}, 96 \%, 46 \%$

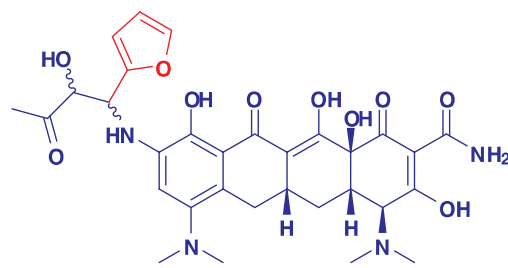

$2 \mathrm{~g}, 99 \%, 66 \%$<smiles>CC(=O)[C@H](O)Cc1ccccc1N[C@H](O)C(=O)O</smiles>

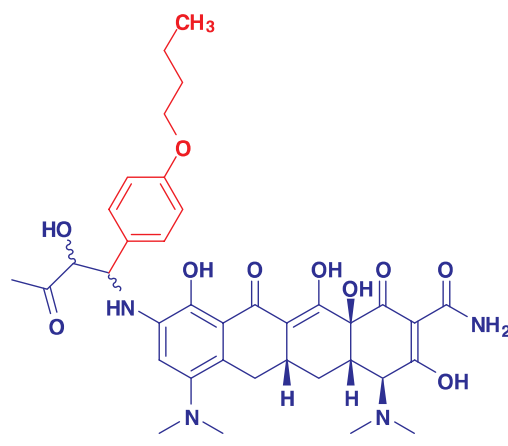

$2 b, 91 \%, 24 \%$

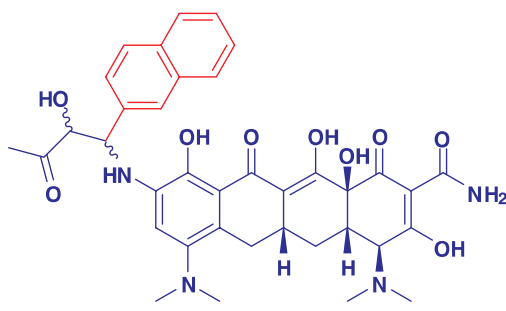

$2 \mathrm{e}, 97 \%, 37 \%$

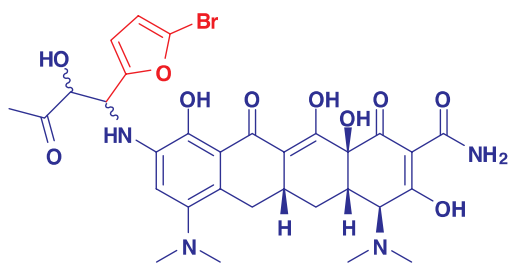

$2 \mathrm{~h}, \mathbf{9 8 \%}, \mathbf{5 0 \%}$

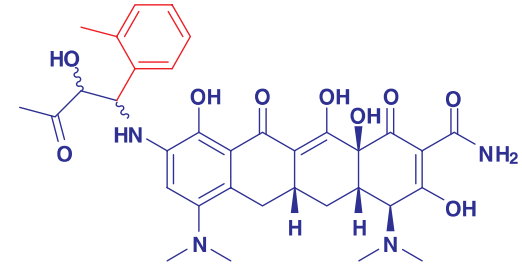

$2 \mathrm{c}, 98 \%, 55 \%$

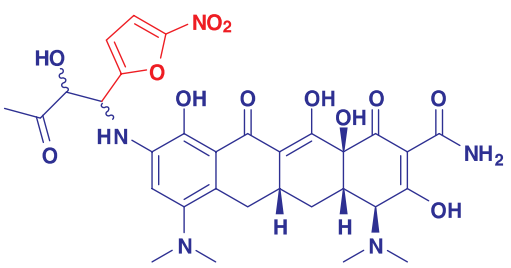

2f, $81 \%, 23 \%$

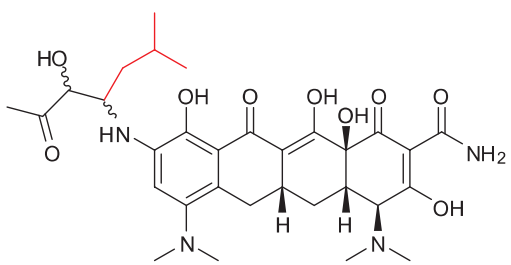

$2 \mathrm{i}, 0 \%, 0 \%$

\section{$2 \mathrm{j}, 0 \%, 0 \%$}

Figure 3 Three-component Mannich reaction with various aldehydes. The compound number is followed by the conversion and isolated yield.

1a. Compound $\mathbf{2 b}$ exhibited an enormous drop in potency for the Gram-negative bacteria. This could be due to the hydrophobicity of the extended carbon chain, which makes it difficult for the compound to pass through the peptidoglycan layer of the cell due to increased hydrophobic interactions. Increasing the bulkiness of the benzene ring had a less significant effect on the potency of this compound as seen by compounds $2 \mathrm{c}$ (methyl group) and $\mathbf{2 e}$ (fused benzene ring). The furfuraldehyde derivatives gave poor structural-activity correlation, as the addition of a substitution group to the furfural ring $(\mathbf{2 f}, \mathbf{2 g}$ and $\mathbf{2 h}$ ) did not have much influence on the MICs.

\section{Conclusions}

In summary, we have shown for the first time that organocatalysis can be employed to synthesize tetracycline derivatives. Although, comparsion to the parent minocycline showed lack of benefit from the addition of the alkylamino side chains at the C9 position, this methodology shows a new and mild synthetic route to derivatize this family of compounds and could be a starting point for future analogues with better biological activity. The Mannich adducts were more active against Gram-positive bacteria than they are with Gram-negatives. The structureactivity relationship studies of these compounds provided use- 
Table 2 Antibacterial activity of Mannich adducts $(n=2)$.

\begin{tabular}{|c|c|c|c|c|}
\hline \multirow[t]{2}{*}{$\begin{array}{l}\text { Product } \\
\text { (see Fig. 1) }\end{array}$} & \multicolumn{2}{|c|}{$\begin{array}{l}\text { Gram-positive bacteria } \\
\qquad / \mu \mathrm{g} \mathrm{mL} \mathrm{m}^{-1}\end{array}$} & \multicolumn{2}{|c|}{$\begin{array}{l}\text { Gram-negative bacteria } \\
\qquad / \mu \mathrm{g} \mathrm{mL}^{-1}\end{array}$} \\
\hline & $\begin{array}{c}\text { S. aureus } \\
\text { ATCC } 25923\end{array}$ & $\begin{array}{l}\text { B. subtilis } \\
\text { ATCC } 6051\end{array}$ & $\begin{array}{c}\text { E. coli } \\
\text { ATCC } 25922\end{array}$ & $\begin{array}{l}\text { P. aeruginosa } \\
\text { ATCC } 27853\end{array}$ \\
\hline Tigecycline & 0.25 & 0.06 & 0.25 & 16 \\
\hline Doxycycline & 0.25 & 0.06 & 0.25 & 16 \\
\hline $1 a$ & 32 & 8 & 32 & NI \\
\hline $1 b$ & 32 & 8 & 128 & NI \\
\hline 1c & 16 & 16 & 32 & NI \\
\hline $1 d$ & 128 & 16 & NI & NI \\
\hline $2 a$ & 8 & 2 & 4 & NI \\
\hline $2 b$ & 4 & 2 & 128 & NI \\
\hline $2 c$ & 16 & 4 & 32 & NI \\
\hline $2 d$ & 32 & 4 & 16 & NI \\
\hline $2 e$ & 32 & 4 & 16 & NI \\
\hline $2 f$ & 8 & 2 & 16 & NI \\
\hline $2 \mathrm{~g}$ & 8 & 1 & 16 & NI \\
\hline $2 \mathrm{~h}$ & 4 & 2 & 8 & $\mathrm{NI}$ \\
\hline
\end{tabular}

ful information on the structural requirements for activity against Gram-negative bacteria. It also indicated that it is possible to design compounds with selectivity just against Gram-positive bacteria. The promising in vitro activity of some of the compounds (e.g. 2a and $2 \mathrm{~g}$ ) makes them potential candidates for the development of new antibiotics selectively targeting Gram-positive pathogens.

\section{Experimental Section}

\subsection{General Experimental Procedures}

Reagents and solvents were purchased from Sigma-Aldrich and were used without purification unless otherwise stated. High resolution mass spectrometric data were obtained with a Bruker micrOTOF-Q II instrument that operated at ambient temperatures and at a sample concentration of $c a .1 .0 \mathrm{ppm}$. NMR spectra were recorded with Bruker AVANCE III 400 or $600 \mathrm{MHz}$ instruments at room temperature. Chemical shifts are expressed ppm, the deuterated solvent reference peak were used as an internal standard for autocalibration.

\subsection{General Experimental Procedure for the Synthesis of Mannich Products from Hydroxyacetone}

To a suspension of L-proline $(4.2 \mathrm{mg}, 0.3 \mathrm{mmol})$ in methanol (2.0 mL), 9-amino minocycline hydrochloride (60 mg, $1.1 \mathrm{mmol})$ was added. To this mixture, the aldehyde $(1.0 \mathrm{mmol})$ followed by the ketone $(1.0 \mathrm{~mL})$ was added and stirred at room temperature for overnight. The reactions were then directly purified straight using semi-preparative reverse-phase HPLC.

\subsection{Chromatography Method}

Semi-preparative reverse-phase HPLC (Shimadzu, Japan) was conducted using a ACE C18 preparative column $(150 \times 21.2 \mathrm{~mm})$ using gradient of $0 \%$ B for $5 \mathrm{~min}, 0 \% \mathrm{~B}$ to $85 \%$ B over $55 \mathrm{~min}$, $85 \%$ B to $95 \%$ B over $1 \mathrm{~min}, 95 \%$ B for $5 \mathrm{~min}, 95 \%$ B to $0 \%$ B over $1 \mathrm{~min}, 0 \% \mathrm{~B}$ for $5 \min \left(\mathrm{A}=\mathrm{dH}_{2} \mathrm{O}\right.$ with $0.1 \% \mathrm{FA}$; $\mathrm{B}=$ Methanol with $0.1 \% \mathrm{FA}$; flow rate $\left.=15 \mathrm{~mL} \mathrm{~min}^{-1} ; \mathrm{A}_{254} \mathrm{~nm}\right)$. The fractions were characterized by LCMS (Shimadzu, Japan). Fractions which showed desired mass were collected and concentrated under reduced pressure and lyophilized to give the products as dark red powders. The products are hygroscopic and also decompose upon heating, therefor melting points were not obtained.

\subsection{Antibacterial Assays}

MICs of the tested compounds were determined by broth microdilution, according to CLSI guidelines. Briefly, two fold dilutions of each inhibitor was made in cation adjusted MuellerHinton Broth (CAMHB) in a microtitre plate. A $10 \mathrm{~mL}$ of 0.5 McFarland bacteria inoculum was added to make a total volume of $100 \mathrm{ml}$ in each microtitre well. Plates were then incubated at $37^{\circ}$ for $18-22 \mathrm{~h}$ under aerobic conditions. MICs were defined at the lowest concentration of antimicrobial agent inhibiting visible growth using the Alamar blue assay. S. aureus ATCC 25923, B. subtilis ATCC 6051, E. coli ATCC 25922 and P. aeruginosa ATCC 27853 were used in this study. Control wells of the amount of DMSO used were also done. Assays were done in duplicate to confirm results. ${ }^{26}$

\subsection{NMR Data}

$\mathrm{OH}$ and $\mathrm{NH}$ protons did not show on the NMR spectra.

(4S,4aS,5aR,12aS)-4,7-bis(dimethylamino)-3,10,12,12a-tetrahydroxy-9-(2-hydroxy-3-oxo-1-phenylbutylamino)-1,11-dioxo1,4,4a,5,5a,6,11,12a-octahydrotetracene-2-carboxamide (1a) ${ }^{1} \mathrm{H}$ NMR $\left(600 \mathrm{MHz}, \mathrm{CD}_{3} \mathrm{OD}+0.1 \%\right.$ TFA) 7.45-7.36 (m, 2H), 7.33-7.28 (m, 1H), 7.28-7.23 (m, 1H), 7.23-7.18 (m, 1H), 6.88-6.75 $(\mathrm{m}, 1 \mathrm{H}), 4.89-4.74(\mathrm{~m}, 1 \mathrm{H}), 4.52-4.21(\mathrm{~m}, 1 \mathrm{H}), 3.23-2.82(\mathrm{~m}, 14 \mathrm{H})$, 2.37-2.21 (m, 3H), 2.21-2.13 (m, 1H), 2.08-1.91 (m, 2H), 1.66-147 $(\mathrm{m}, 1 \mathrm{H}), 1.32-1.24(\mathrm{~m}, 1 \mathrm{H}),\left(\mathrm{OH}^{\prime} \mathrm{s}, 5, \mathrm{NH}^{\prime} \mathrm{s}, 3\right)$. HRMS $\left(\mathrm{ESI}^{+}\right)$: calcd. for $\mathrm{C}_{33} \mathrm{H}_{39} \mathrm{~N}_{4} \mathrm{O}_{9}[\mathrm{M}+\mathrm{H}]^{+}$635.2712; found 635.2724.

(4S,4aS,5aR,12aS)-4,7-bis(dimethylamino)-3,10,12,12a-tetrahydroxy-9-(2-methyl-3-oxo-1-phenylbutylamino)-1,11-dioxo-1 ,4,4a,5,5a,6,11,12a-octahydrotetracene-2-carboxamide (1b) ${ }^{1} \mathrm{H}$ NMR $\left(600 \mathrm{MHz}, \mathrm{CD}_{3} \mathrm{OD}+0.1 \%\right.$ TFA) 7.45-7.35 (m, 2H), 7.34-7.26 (m, 2H), 7.24-7.18 (m, 1H), 6.75-6.68 (m, 1H), 4.85-4.78 $(\mathrm{m}, 1 \mathrm{H}), 4.11-3.97(\mathrm{~m}, 1 \mathrm{H}), 3.17-2.80(\mathrm{~m}, 17 \mathrm{H}), 2.52-2.23(\mathrm{~m}, 2 \mathrm{H})$, $2.20-2.16(\mathrm{~m}, 1 \mathrm{H}), 2.00-1.96(\mathrm{~m}, 1 \mathrm{H}), 1.64-1.48(\mathrm{~m}, 1 \mathrm{H}), 1.28-1.22$ (m, 1H), 1.08-1.00 (m, 1H), 1.00-0.906 (m, 1H), (OH's, 4, NH's, 3). HRMS (ESI ${ }^{+}$): calcd. for $\mathrm{C}_{34} \mathrm{H}_{41} \mathrm{~N}_{4} \mathrm{O}_{8}[\mathrm{M}+\mathrm{H}]^{+}$633.2919; found 633.2906 .

(4S,4aS,5aR,12aS)-4,7-bis(dimethylamino)-3,10,12,12a-tetrahydroxy-1,11-dioxo-9-(3-oxo-1-phenylbutylamino)-1,4,4a,5,5a, 6,11,12a-octahydrotetracene-2-carboxamide (1c)

${ }^{1} \mathrm{H}$ NMR $\left(600 \mathrm{MHz}, \mathrm{CD}_{3} \mathrm{OD}+0.1 \%\right.$ TFA) 7.63-7.59 (m, 2H), $7.41-7.38(\mathrm{~m}, 2 \mathrm{H}), 7.33-7.27(\mathrm{~m}, 1 \mathrm{H}), 6.77-6.70(\mathrm{~m}, 1 \mathrm{H}), 4.867-4.75$ $(\mathrm{m}, 1 \mathrm{H}), 3.25-3.19(\mathrm{~m}, 8 \mathrm{H}), 3.08-2.97(\mathrm{~m}, 12 \mathrm{H}), 2.48-2.38(\mathrm{~m}, 1 \mathrm{H})$, 2.37-2.32 (m, 1H), 2.19-2.10 (m, 2H), (OH's, 4, NH's, 3). HRMS $\left(\right.$ ESI $\left.^{+}\right)$: calcd. for $\mathrm{C}_{33} \mathrm{H}_{39} \mathrm{~N}_{4} \mathrm{O}_{8}[\mathrm{M}+\mathrm{H}]^{+}$619.2762; found 6.19.2751.

(4S,4aS,5aR,12aS)-4,7-bis(dimethylamino)-3,10,12,12a-tetrahydroxy-1,11-dioxo-9-((1R)-(2-oxocyclohexyl)(phenyl)methylamino)-1,4,4a,5,5a,6,11,12a-octahydrotetracene-2-carboxamide (1d)

${ }^{1} \mathrm{H}$ NMR $\left(600 \mathrm{MHz}, \mathrm{CD}_{3} \mathrm{OD}+0.1 \%\right.$ TFA) 7.47-7.40 (m, 2H), $7.36-7.26(\mathrm{~m}, 2 \mathrm{H}), 7.24-7.17(\mathrm{~m}, 1 \mathrm{H}), 6.82-6.75(\mathrm{~m}, 1 \mathrm{H}), 4.90-4.78$ $(\mathrm{m}, 1 \mathrm{H}), 4.12-4.04(\mathrm{~m}, 1 \mathrm{H}), 3.11-2.96(\mathrm{~m}, 14 \mathrm{H}), 2.36-2.28(\mathrm{~m}, 4 \mathrm{H})$, 1.73-1.56 (m, 9H), $\left(\mathrm{OH}^{\prime} \mathrm{s}, 4, \mathrm{NH}^{\prime} \mathrm{s}, 3\right)$. HRMS $\left(\mathrm{ESI}^{+}\right)$: calcd. for $\mathrm{C}_{36} \mathrm{H}_{43} \mathrm{~N}_{4} \mathrm{O}_{8}[\mathrm{M}+\mathrm{H}]^{+}$659.3362; found 659.3352 .

(4S,4aS,5aR,12aS)-4,7-bis(dimethylamino)-3,10,12,12a-tetrahydroxy-9-((1S,2R)-2-hydroxy-1-(4-nitrophenyl)-3-oxobutylamino)-1,11-dioxo-1,4,4a,5,5a,6,11,12a-octahydrotetracene-2carboxamide (2a)

${ }^{1} \mathrm{H}$ NMR (600 MHz, CD $\mathrm{OD}+0.1 \%$ TFA) 8.20-8.15 (m, 2H), $7.72-7.66(\mathrm{~m}, 2 \mathrm{H}), 6.94-6.88(\mathrm{~m}, 1 \mathrm{H}), 4.49-4.45(\mathrm{~m}, 1 \mathrm{H}), 4.11-4.03$ (m, 1H), 3.13-2.98 (m, 14H), 2.37-2.29 (m, 3H), 2.14-2.08 (m, 2H), $1.64-1.53(\mathrm{~m}, 1 \mathrm{H}), 1.33-1.27$ (m, 2H), (OH's, 5, NH's, 3). HRMS 
(ESI ${ }^{+}$): calcd. for $\mathrm{C}_{33} \mathrm{H}_{38} \mathrm{~N}_{5} \mathrm{O}_{11}[\mathrm{M}+\mathrm{H}]^{+}$680.2562; found 680.2554.

(4S,4aS,5aR,12aS)-9-((1S,2R)-1-(4-butoxyphenyl)-2-hydroxy-3oxobutylamino)-4,7 bis(dimethylamino)-3,10,12,12a-tetrahydroxy-1,11-dioxo-1,4,4a,5,5a,6,11,12a-octahydrotetracene-2carboxamide (2b)

${ }^{1} \mathrm{H}$ NMR $\left(600 \mathrm{MHz}, \mathrm{CD}_{3} \mathrm{OD}+0.1 \%\right.$ TFA) 7.36-7.28 (m, 2H), 6.87-6.83 (m, 2H), 6.82-6.77 (m, 1H), 4.43-4.38 (m, 1H), 4.11-4.04 $(\mathrm{m}, 1 \mathrm{H}), 3.93-3.86(\mathrm{~m}, 2 \mathrm{H}), 3.15-2.96(\mathrm{~m}, 15 \mathrm{H}), 2.30-2.23(\mathrm{~m}, 3 \mathrm{H})$, $2.21-2.15(\mathrm{~m}, 1 \mathrm{H}), 2.08-2.03(\mathrm{~m}, 1 \mathrm{H}), 1.71-1.65(\mathrm{~m}, 2 \mathrm{H}), 1.63-1.56$ $(\mathrm{m}, 1 \mathrm{H}), 1.47-1.41(\mathrm{~m}, 2 \mathrm{H}), 1.39-1.36(\mathrm{~m}, 1 \mathrm{H}), 0.95-0.90(\mathrm{~m}, 3 \mathrm{H})$, $\left(\mathrm{OH}^{\prime} \mathrm{s}, 5, \mathrm{NH}^{\prime} \mathrm{s}, 3\right)$. HRMS $\left(\mathrm{ESI}^{+}\right)$: calcd. for $\mathrm{C}_{37} \mathrm{H}_{47} \mathrm{~N}_{4} \mathrm{O}_{10}[\mathrm{M}+\mathrm{H}]^{+}$ 707.3287; found 707.3270.

(4S,4aS,5aR,12aS)-4,7-bis(dimethylamino)-3,10,12,12a-tetrahydroxy-9-((1S,2R)-2-hydroxy-3-oxo-1-o-tolylbutylamino)-1,1 1-dioxo-1,4,4a,5,5a,6,11,12a-octahydrotetracene-2-carboxamide (2c)

${ }^{1} \mathrm{H}$ NMR (600 MHz, CD $\mathrm{OD}+0.1 \%$ TFA) 7.23-7.17 (m, 2H), $7.14-7.11(\mathrm{~m}, 1 \mathrm{H}), 7.09-7.05(\mathrm{~m}, 1 \mathrm{H}), 6.51-6.48(\mathrm{~m}, 1 \mathrm{H}), 4.90-4.74$ $(\mathrm{m}, 1 \mathrm{H}), 4.392-4.35(\mathrm{~m}, 1 \mathrm{H}), 3.05-2.96(\mathrm{~m}, 15 \mathrm{H}), 2.59-2.56(\mathrm{~m}, 3 \mathrm{H})$, 2.51-2.47 (m, 1H), 2.35-2.32 (m, 3H), 1.99-1.97 (m, 1H), 1.63-1.53 $(\mathrm{m}, 2 \mathrm{H}),\left(\mathrm{OH}^{\prime} \mathrm{s}, 5, \mathrm{NH}^{\prime} \mathrm{s}, 3\right)$. HRMS $\left(\mathrm{ESI}^{+}\right)$: calcd. for $\mathrm{C}_{34} \mathrm{H}_{41} \mathrm{~N}_{4} \mathrm{O}_{9}$ $[\mathrm{M}+\mathrm{H}]^{+}$649.2868; found 649.2866.

(4S,4aS,5aR,12aS)-4,7-bis(dimethylamino)-9-((1S,2R)-1-(4fluorophenyl)-2-hydroxy-3-oxobutylamino)-3,10,12,12a-tetrahydroxy-1,11-dioxo-1,4,4a,5,5a,6,11,12a-octahydrotetracene-2carboxamide (2d)

${ }^{1} \mathrm{H}$ NMR $\left(600 \mathrm{MHz}, \mathrm{CD}_{3} \mathrm{OD}+0.1 \%\right.$ TFA) 7.52-7.41 (m, 2H), 7.10-6.97 (m, 2H), 6.91-6.81 (m, 1H), 4.47-4.37 (m, 1H), 4.18-4.01 $(\mathrm{m}, 1 \mathrm{H}), 3.21-2.92(\mathrm{~m}, 17 \mathrm{H}), 2.35-2.29(\mathrm{~m}, 3 \mathrm{H}), 2.10-2.06(\mathrm{~m}, 1 \mathrm{H})$, 1.68-1.55 (m, 1H), (OH's, 5, NH's, 3). HRMS (ESI $\left.{ }^{+}\right)$: calcd. for $\mathrm{C}_{33} \mathrm{H}_{38} \mathrm{FN}_{4} \mathrm{O}_{9}[\mathrm{M}+\mathrm{H}]^{+}$653.2617; found 653.2592 .

(4S,4aS,5aR,12aS)-4,7-bis(dimethylamino)-3,10,12,12a-tetrahydroxy-9-((1S,2R)-2-hydroxy-1-(naphthalen-2-yl)-3-oxobutyl amino)-1,11-dioxo-1,4,4a,5,5a,6,11,12a-octahydrotetracene-2carboxamide (2e)

1H NMR (600 MHz, CD3OD + $0.1 \%$ TFA) 7.93-7.90 (m, 1H), $7.80-7.78(\mathrm{~m}, 2 \mathrm{H}), 7.61-7.58(\mathrm{~m}, 1 \mathrm{H}), 7.45-7.40(\mathrm{~m}, 3 \mathrm{H}), 6.91-6.88$ $(\mathrm{m}, 1 \mathrm{H}), 4.85-4.79(\mathrm{~m}, 1 \mathrm{H}), 4.56-4.52(\mathrm{~m}, 1 \mathrm{H}), 3.04-2.95(\mathrm{~m}, 14 \mathrm{H})$, $2.35-2.32(\mathrm{~m}, 3 \mathrm{H}), 2.25-2.22(\mathrm{~m}, 1 \mathrm{H}), 2.19-2.16(\mathrm{~m}, 1 \mathrm{H})$ 2.078-2.036 (m, 1H), 2.02-2.00 (m, 1H), 1.59-1.53 (m, 1H), (OH's, 5, $\mathrm{NH}^{\prime}$ s, 3) . HRMS $\left(\right.$ ESI $\left.^{+}\right)$: calcd. for $\mathrm{C}_{37} \mathrm{H}_{41} \mathrm{~N}_{4} \mathrm{O}_{9}[\mathrm{M}+\mathrm{H}]^{+}$ 685.2868; found 685.2865 .

(4S,4aS,5aR,12aS)-4,7-bis(dimethylamino)-3,10,12,12a-tetrahydroxy-9-((1R,2R)-2-hydroxy-1-(5-nitrofuran-2-yl)-3-oxobutylamino)-1,11-dioxo-1,4,4a,5,5a,6,11,12a-octahydrotetracene-2-carboxamide (2f)

${ }^{1} \mathrm{H}$ NMR $\left(600 \mathrm{MHz}, \mathrm{CD}_{3} \mathrm{OD}+0.1 \%\right.$ TFA) 7.41-7.27 (m, 2H), 6.73-6.65 (m, 1H), 4.90-4.80 (m, 1H), 4.74-4.65 (m, 1H), 3.28-3.18 (m, 10H), 3.08-3.00 (m, 7H), 2.34-2.30 (m, 3H), 1.72-1.58 (m, 2H), $\left(\mathrm{OH}^{\prime} \mathrm{s}, 5, \mathrm{NH}^{\prime} \mathrm{s}, 3\right)$. HRMS (ESI $\left.{ }^{+}\right)$: calcd. for $\mathrm{C}_{31} \mathrm{H}_{36} \mathrm{~N}_{5} \mathrm{O}_{12}[\mathrm{M}+\mathrm{H}]^{+}$ 670.2355; found 670.2322 .

(4S,4aS,5aR,12aS)-4,7-bis(dimethylamino)-9-((1R,2R)-1-(fura-2 -yl)-2-hydroxy-3-oxobutylamino)-3,10,12,12a-tetrahydroxy-1, 11-dioxo-1,4,4a,5,5a,6,11,12a-octahydrotetracene-2-carboxamide (2g)

${ }^{1} \mathrm{H}$ NMR $\left(600 \mathrm{MHz}, \mathrm{CD}_{3} \mathrm{OD}+0.1 \%\right.$ TFA) 7.51-7.35 (m, 1H), 7.17-7.05 (m, 1H), 6.38-6.23 (m, 2H), 4.86-4.80 (m, 1H), 4.65-4.56 $(\mathrm{m}, 1 \mathrm{H}), 3.22-3.15(\mathrm{~m}, 10 \mathrm{H}), 3.09-2.98(\mathrm{~m}, 7 \mathrm{H}), 2.41-2.32(\mathrm{~m}, 1 \mathrm{H})$, $2.30-2.24(\mathrm{~m}, 2 \mathrm{H}), 2.22-2.19(\mathrm{~m}, 1 \mathrm{H}), 1.63-1.57(\mathrm{~m}, 1 \mathrm{H}),\left(\mathrm{OH}^{\prime} \mathrm{s}, 4\right.$, $\mathrm{NH}^{\prime}$ s, 3) . HRMS $\left(\mathrm{ESI}^{+}\right)$: calcd. for $\mathrm{C}_{31} \mathrm{H}_{37} \mathrm{~N}_{4} \mathrm{O}_{10}[\mathrm{M}+\mathrm{H}]^{+}$625.2504; found 625.2486 .
(4S,4aS,5aR,12aS)-9-(1-(5-bromofuran-2-yl)-2-hydroxy-3-oxobutylamino)-4,7-bis (dimethylamino)-3,10,12,12a-tetrahydroxy-1,11-dioxo-1,4,4a,5,5a,6,11,12a-octahydrotetracene-2carboxamide $(\mathbf{2 h})$

${ }^{1} \mathrm{H}$ NMR $\left(600 \mathrm{MHz}, \mathrm{CD}_{3} \mathrm{OD}+0.1 \%\right.$ TFA) 7.22-7.08 (m, 1H), 6.39-6.20 (m, 2H), 4.87-4.78 (m, 1H), 4.61-4.52 (m, 1H), 3.27-3.11 $(\mathrm{m}, 10 \mathrm{H}) 3.09-2.92(\mathrm{~m}, 7 \mathrm{H}), 2.44-2.33(\mathrm{~m}, 1 \mathrm{H}), 2.30-2.21(\mathrm{~m}, 3 \mathrm{H})$, 1.67-1.54 (m, 1H), $\left(\mathrm{OH}^{\prime} \mathrm{s}, 5, \mathrm{NH}^{\prime} \mathrm{s}, 3\right)$. HRMS $\left(\mathrm{ESI}^{+}\right)$: calcd. for $\mathrm{C}_{31} \mathrm{H}_{36} \mathrm{Br} \mathrm{N}_{4} \mathrm{O}_{10}[\mathrm{M}+\mathrm{H}]^{+}$703.1609; found 703.1588 .

\section{Acknowledgements}

This study was supported by College of Health Science, University of Kwa-Zulu Natal, Durban, South Africa; Aspen Pharmacare, South Africa, and the South African National Research Foundation.

\section{Supplementary Material}

For copies of proton NMR spectra, HPLC purity chromatographs and high-resolution mass spectra.

\section{References}

1 B.M. Duggar, Aureomycin: a product of the continuing search for new antibiotics, Ann. N.Y. Acad. Sci., 1948, 51, 177-181.

2 a) I. Chopra, P. Hawkey and M. Hinton, Tetracyclines, molecular and clinical aspects, J. Antimicrob. Chemother., 1992, 29, 245-277; b) D. Schnappinger, W. Hillen, Tetracyclines: antibiotic action, uptake, and resistance mechanisms, Arch. Microbiol., 1996, 165, 359-369.

3 A. Parisien, B. Allain, J. Zhang, R. Mandeville and C. Lan, Novel alternatives to antibiotics: bacteriophages, bacterial cell wall hydrolases, and antimicrobial peptides, J. Appl. Microbiol., 2008, 104, 1-13.

4 P.J. Petersen, N. Jacobus, W. Weiss, P. Sum and R. Testa, In vitro and in vivo antibacterial activities of a novel glycylcycline, the 9-t-butylglycylamido derivative of minocycline (GAR-936), Antimicrob. Agents Chemother., 1999, 43, 738-744.

5 a) M.G. Charest, D. R. Siegel and A.G. Myers, Synthesis of (-)-tetracycline, J. Am. Chem. Soc., 2005, 127, 8292-8293; b) Y. Wang, R. Castaner, J. Bolos and C. Estivill, Amadacycline tetracycline antibiotic, Drugs of the Future, 2009, 34, 11-15; c) F. Nguyen, A.L. Starosta, S. Arenz, D. Sohmen, A. Dönhöfer and D.N. Wilson, Tetracycline antibiotics and resistance mechanisms, Biol. Chem., 2014, 395, 559-575.

6 a) X.-Y. Xiao, D.K. Hunt, J. Zhou, R.B. Clark, N. Dunwoody, C. Fyfe, T.H. Grossman, W.J. O’Brien, L. Plamondon and M. Rönn, Fluorocyclines. 1. 7-Fluoro-9-pyrrolidinoacetamido-6-demethyl-6-deoxytetracycline: a potent, broad spectrum antibacterial agent, J. Med. Chem., 2012, 55, 597-605; b) C. Sun, D.K. Hunt, R.B. Clark, D. Lofland, W.J. O'Brien, L. Plamondon and X.-Y. Xiao, Synthesis and antibacterial activity of pentacyclines: a novel class of tetracycline analogs, $J$. Med. Chem., 2011, 54, 3704-3731.

7 P.-E. Sum, A.T. Ross, P.J. Petersen and R.T. Testa, Synthesis and antibacterial activity of 9-substituted minocycline derivatives, Bioorg. Med. Chem. Lett., 2006, 16, 400-403.

8 a) K. Nicolaou, C.R. Hale, C. Nilewski, H.A. Ioannidou, A. ElMarrouni, L.G. Nilewski, K. Beabout, T.T. Wang and Y. Shamoo, Total synthesis of viridicatumtoxin B and analogues thereof: strategy evolution, structural revision, and biological evaluation, J. Am. Chem. Soc., 2014, 136, 12137-12160; b) C. Sun, D.K. Hunt, C.-L. Chen, Y. Deng, M. He, R.B. Clark, C. Fyfe, T.H. Grossman, J.A. Sutcliffe and X.-y.C. Xiao, Design, synthesis, and biological evaluation of hexacyclic tetracyclines as potent, broad spectrum antibacterial agents, J. Med. Chem., 2015; c) U. Lešnik, T. Lukežič, A. Podgoršek, J. Horvat, T. Polak, M. Šala, B. Jenko, K. Harmrolfs, A. Ocampo-Sosa and L. Martínez-Martínez, Construction of a new class of tetracycline lead structures with potent antibacterial activity through biosynthetic engineering, Angew. Chem. Int. Ed., 2015, 54, 3937-3940.

9 A. Ricci, Asymmetric Organocatalysis at the service of medicinal chemistry, ISRN. Org. Chem., 2014, 2014.

10 B. List, R.A. Lerner and C.F. Barbas, Proline-catalyzed direct asymmetric aldol reactions, J. Am. Chem. Soc., 2000, 122, 2395-2396.

11 a) B. List, P. Pojarliev, W.T. Biller and H.J. Martin, The prolinecatalyzed direct asymmetric three-component Mannich reaction: scope, optimization, and application to the highly enantioselective synthesis of 1, 2-amino alcohols, J. Am. Chem. Soc., 2002, 124, 827- 
H.G. Kruger, T. Govender and T. Naicker

S. Afr. J. Chem., 2016, 69, 72-78,

$<$ http://journals.sabinet.co.za/sajchem/>.

833; b) Y. Xue, L.-P. Li, Y.-H. He and Z. Guan, Protease-catalysed direct asymmetric Mannich reaction in organic solvent, Sci. Rep., $2012,2$.

12 a) S. Sulzer-Mosse and A. Alexakis, Chiral amines as organocatalysts for asymmetric conjugate addition to nitroolefins and vinyl sulfones via enamine activation, Chem. Commun., 2007, 3123-3135; b) B. List, P. Pojarliev and H.J. Martin, Efficient proline-catalyzed Michael additions of unmodified ketones to nitro olefins, Org. Lett., 2001, 3, 2423-2425.

13 a) W. Notz, F. Tanaka and C.F. Barbas, Enamine-based organocatalysis with proline and diamines: the development of direct catalytic asymmetric Aldol, Mannich, Michael, and Diels-Alder reactions, Acc. Chem. Res., 2004, 37, 580-591; b) E. Zamani, Organocatalyzed stereospecific $\mathrm{C}-\mathrm{C}$ bond formation of $\beta$-lactams, Org. Biomol. Chem. 2013, 11, 8294-8297.

14 a) J. Alemán and S. Cabrera, Applications of asymmetric organocatalysis in medicinal chemistry, Chem. Soc. Rev., 2013, 42, 774-793; b) M. Raj and V.K. Singh, Recent advances on stereoselective organocatalytic reactions. Organocatalytic synthesis of natural products and drugs, Catalytic Methods in Asymmetric Synthesis: Advanced Materials, Techniques, and Applications, 2011, 415.

15 a) Z.E. Cele, S.A. Pawar, T. Naicker, G.E. Maguire, P.I. Arvidsson, H.G. Kruger and T. Govender, Organocatalytic Mannich reactions on a carbapenem core - synthesis of Mannich bases and bicyclic diazanonanes, Eur. J. Org. Chem., 2014, 2014, 2253-2260; b) Z.E. Cele, P.I. Arvidsson, H.G. Kruger, T. Govender and T. Naicker, Applied enantioselective aminocatalysis: $\alpha$-heteroatom functionalization reactions on the carbapenem ( $\beta$-lactam antibiotic) core, Eur. J. Org. Chem., 2015, 2015, 638-646; c) S. Khanyase, T. Naicker, G.E. Maguire, H.G. Kruger, P.I. Arvidsson and T. Govender, l-Proline organocatalyzed Michael synthesis of monobactam and carbapenem $\beta$-lactam cores, Tetrahedron: Asymmetry, 2014, 25, 969-973.

16 S. Bala, N. Sharma, A. Kajal, S. Kamboj and V. Saini, Mannich Bases: An important pharmacophore in present scenario, Int. J. Med. Chem., 2014,2014
17 W. Siedel, A. Soder and F. Lindner, Die Aminomethylierung der Tetracycline. Zur Chemie des Reverin, Munch. Med. Wochenschr., 1958, 17, 661-663.

18 K.M Huttunen and J. Rautio, Prodrugs - an efficient way to breach delivery and targeting barriers, Curr. Top. Med. Chem., 2011, 11, 2265-2287.

19 A.L. Simplício, J.M. Clancy and J.F. Gilmer, $\beta$-Aminoketones as prodrugs with $\mathrm{pH}$-controlled activation, Int. J. Pharm., 2007, 336, 208-214

20 A. Córdova, The direct catalytic asymmetric Mannich reaction, Acc. Chem. Res., 2004, 37, 102-112.

21 P.I. Dalko, Enantioselective Organocatalysis, Wiley Online Library, 2007.

22 B.T. Hahn, R. Froehlich, K. Harms and F. Glorius, Proline-catalyzed highly enantioselective and anti-selective Mannich reaction of unactivated ketones: synthesis of chiral $\alpha$-amino acids, Angew. Chem. Int. Ed., 2008, 47, 9985-9988.

23 a) M.M. Salter, J. Kobayashi, Y. Shimizu and S. Kobayashi, Direct-type catalytic three-component mannich reactions leading to an efficient synthesis of $\alpha, \beta$-diamino acid derivatives, Org. Lett., 2006, 8, 3533-3536; b) B. List, Proline-catalyzed asymmetric reactions, Tetrahedron, 2002, 58, 5573-5590.

24 a) C. Sun, Q. Wang, J. D. Brubaker, P.M. Wright, C.D. Lerner K. Noson, M. Charest, D.R. Siegel, Y.-M. Wang and A.G. Myers, A robust platform for the synthesis of new tetracycline antibiotics, J. Am. Chem. Soc., 2008, 130, 17913-17927; b) M.L. Nelson, M.Y. Ismail, L. McIntyre, B. Bhatia, P. Viski, P. Hawkins, G. Rennie, D. Andorsky, D. Messersmith and K. Stapleton, Versatile and facile synthesis of diverse semisynthetic tetracycline derivatives via Pd-catalyzed reactions, J. Org. Chem.,2003, 68, 5838-5851.

25 Y. Takeuchi, Y. Imafuku and M. Nishikawa, Reassignment of the 13C NMR spectrum of minomycin, Arkivoc, 2003, 15, 39-46.

26 Clinical and Laboratory Standards Institute, Perfomance Standard for Antimicrobial Susceptibility Testing; Twenty-fourth Informational Supplement 34, 2014 
Organocatalyzed Mannich Reactions on Minocycline: Towards Novel Tetracycline Antibiotics

Tirivashe E. Chiwunze ${ }^{[a]}$, Rafiatu Azumah ${ }^{[a]}$, Melissa Ramtahal ${ }^{[a]}$, Per I. Arvidsson ${ }^{[b]}$, Sabiha Y. Essack ${ }^{[c]}$, Hendrik G. Kruger ${ }^{[a]}$, Thavendran Govender ${ }^{[a]}$, Tricia Naicker ${ }^{[a]}$

${ }^{a}$ School of Pharmacy and Pharmacology, University of KwaZulu-Natal, Westville Campus, Durban 4000, South Africa.

*e-mail: Naickert1@ukzn.ac.za>

${ }^{\mathbf{b}}$ Science for Life Laboratory, Drug Discovery and Development Platform, and Division of Translational Medicine and Chemical Biology, Department of Medical Biochemistry and Biophysics, Karolinska Institutet, Stockholm, Sweden

${ }^{\mathrm{c}}$ School of Chemistry and Physics, University of KwaZulu-Natal, Westville Campus, Durban 4000, South Africa

\section{SUPPLEMETARY DATA}




\section{Contents}

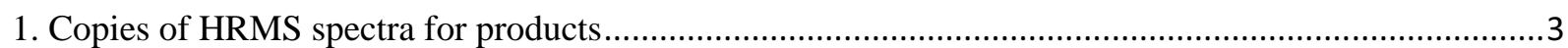

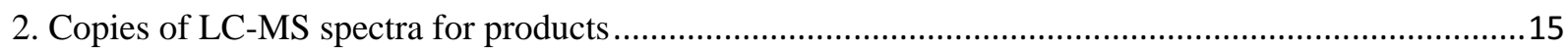

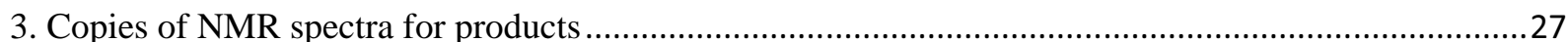




\section{Copies of HRMS spectra for products}

HRMS of (4S,4aS,5aR,12aS)-4,7-bis(dimethylamino)-3,10,12,12a-tetrahydroxy-9-(2hydroxy-3-oxo-1-phenylbutylamino)-1,11-dioxo-1,4,4a,5,5a,6,11,12a-octahydrotetracene-2carboxamide (1a)

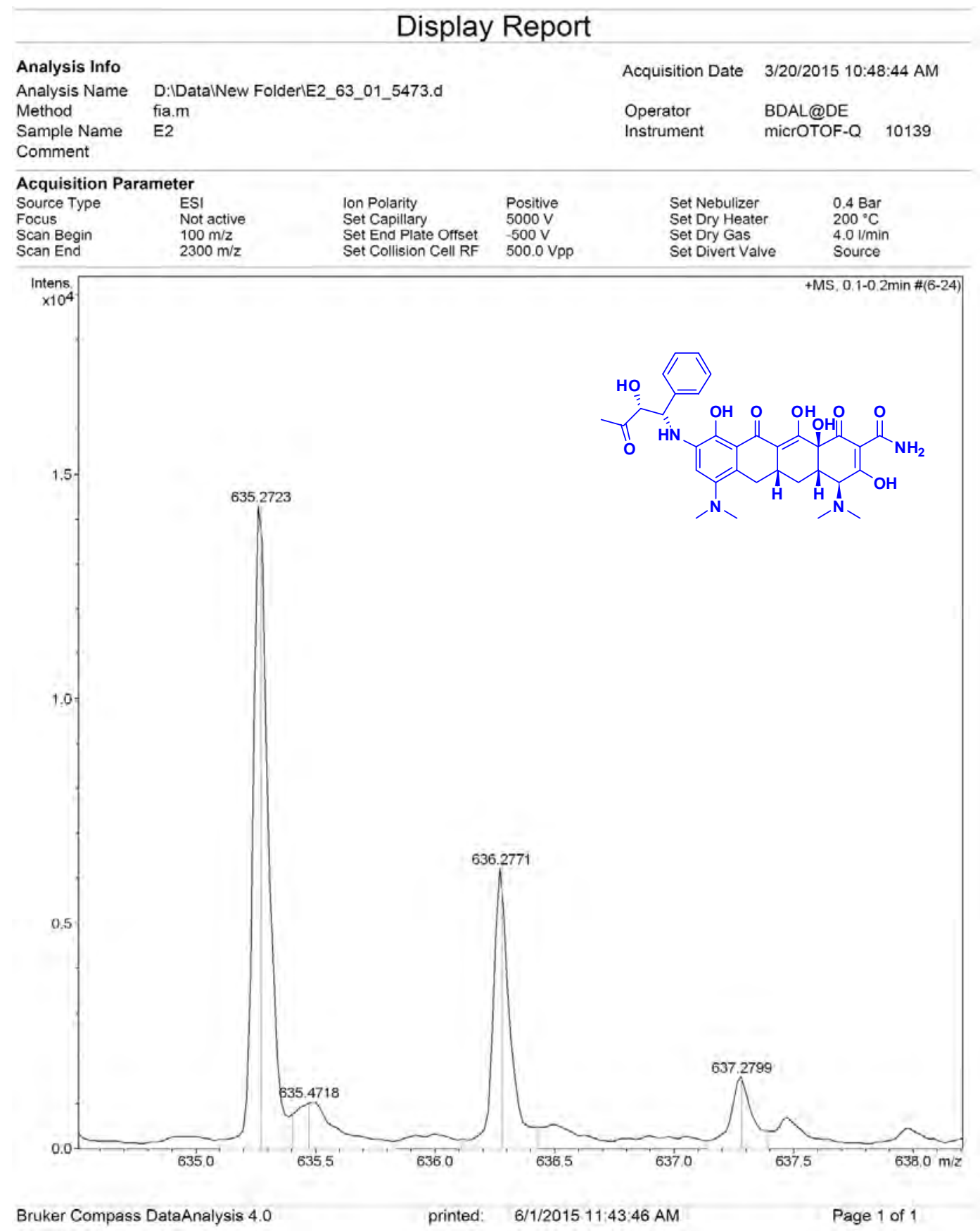


HRMS of (4S,4aS,5aR,12aS)-4,7-bis(dimethylamino)-3,10,12,12a-tetrahydroxy-9-(2methyl-3-oxo-1-phenylbutylamino)-1,11-dioxo-1,4,4a,5,5a,6,11,12a-octahydrotetracene-2carboxamide (1b)

\section{Display Report}

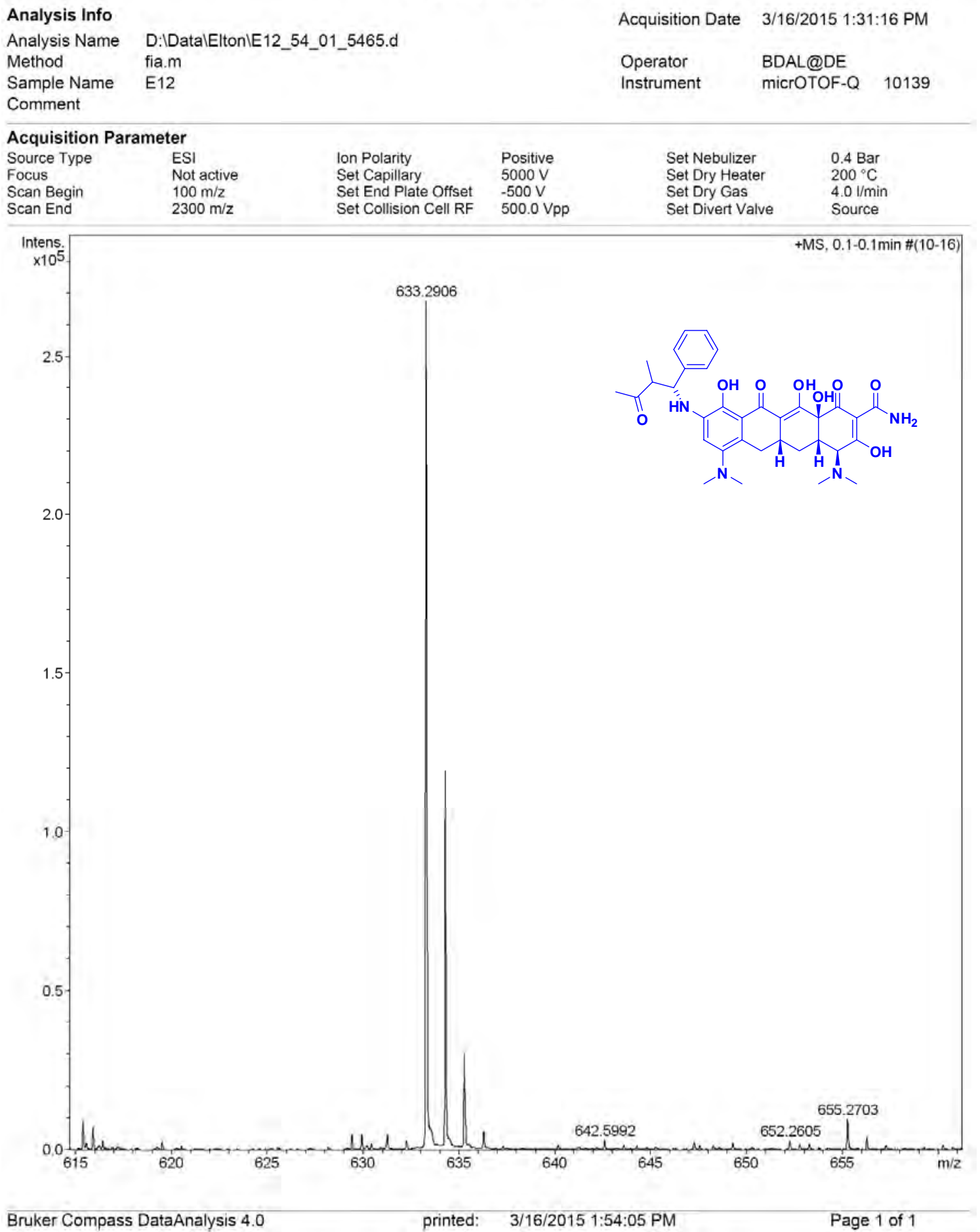


HRMS of (4S,4aS,5aR,12aS)-4,7-bis(dimethylamino)-3,10,12,12a-tetrahydroxy-1,11-dioxo-9(3-oxo-1-phenylbutylamino)-1,4,4a,5,5a,6,11,12a-octahydrotetracene-2-carboxamide (1c)

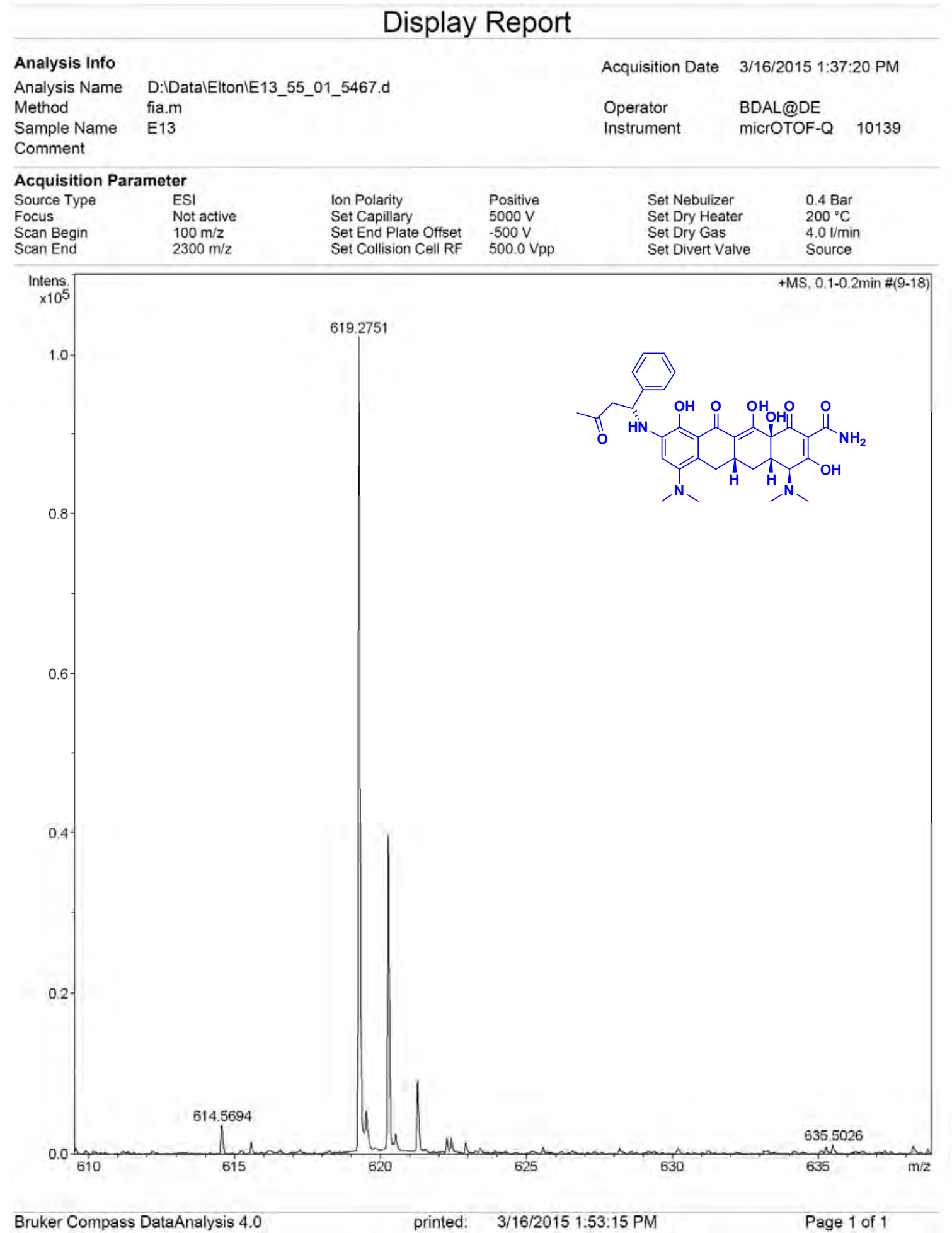


HRMS of (4S,4aS,5aR,12aS)-4,7-bis(dimethylamino)-3,10,12,12a-tetrahydroxy-1,11-dioxo-9((1R)-(2-oxocyclohexyl)(phenyl)methylamino)-1,4,4a,5,5a,6,11,12a-octahydrotetracene-2carboxamide (1d)

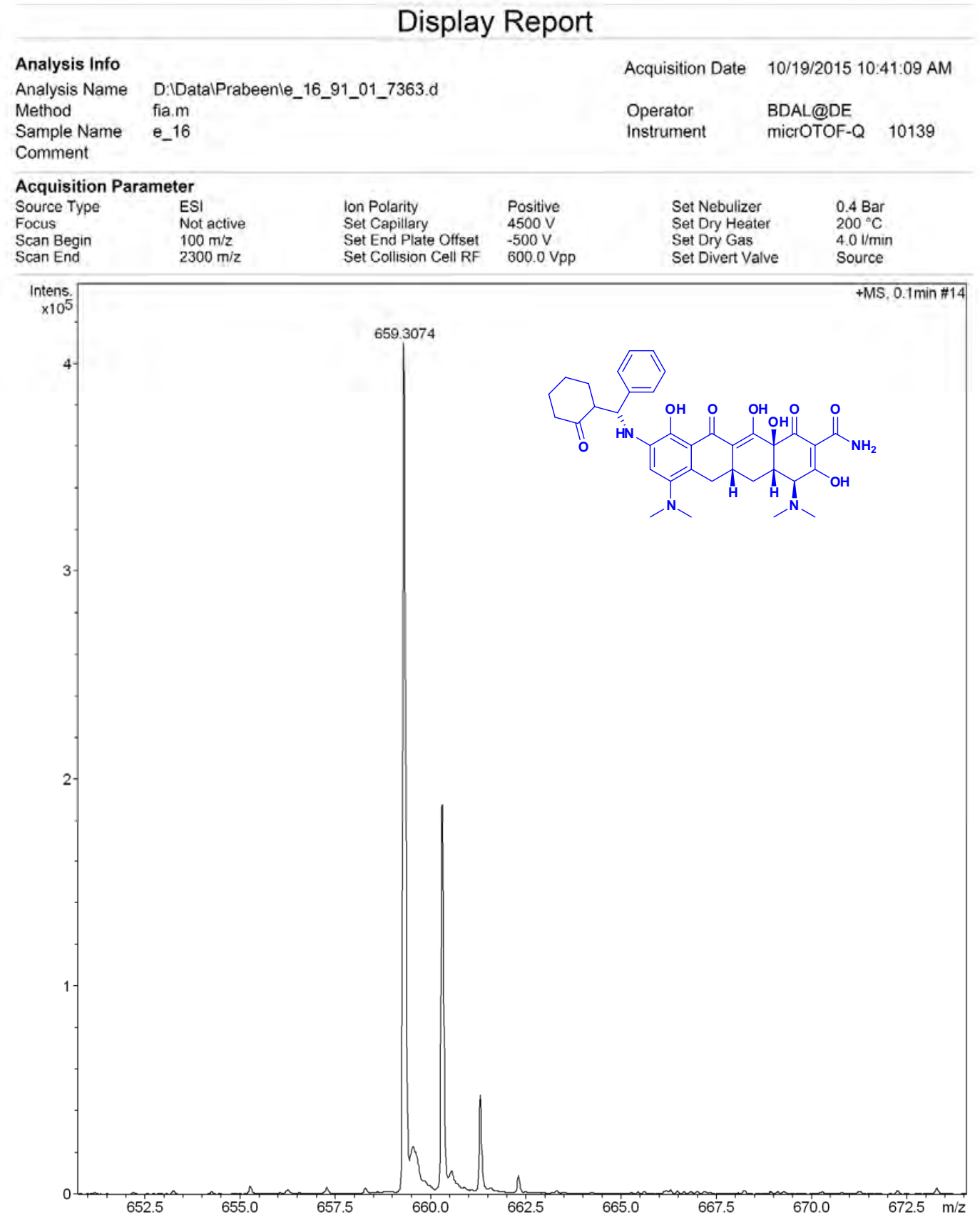


HRMS of (4S,4aS,5aR,12aS)-4,7-bis(dimethylamino)-3,10,12,12a-tetrahydroxy-9-((1S,2R)2-hydroxy-1-(4-nitrophenyl)-3-oxobutylamino)-1,11-dioxo-1,4,4a,5,5a,6,11,12aoctahydrotetracene-2-carboxamide (2a)

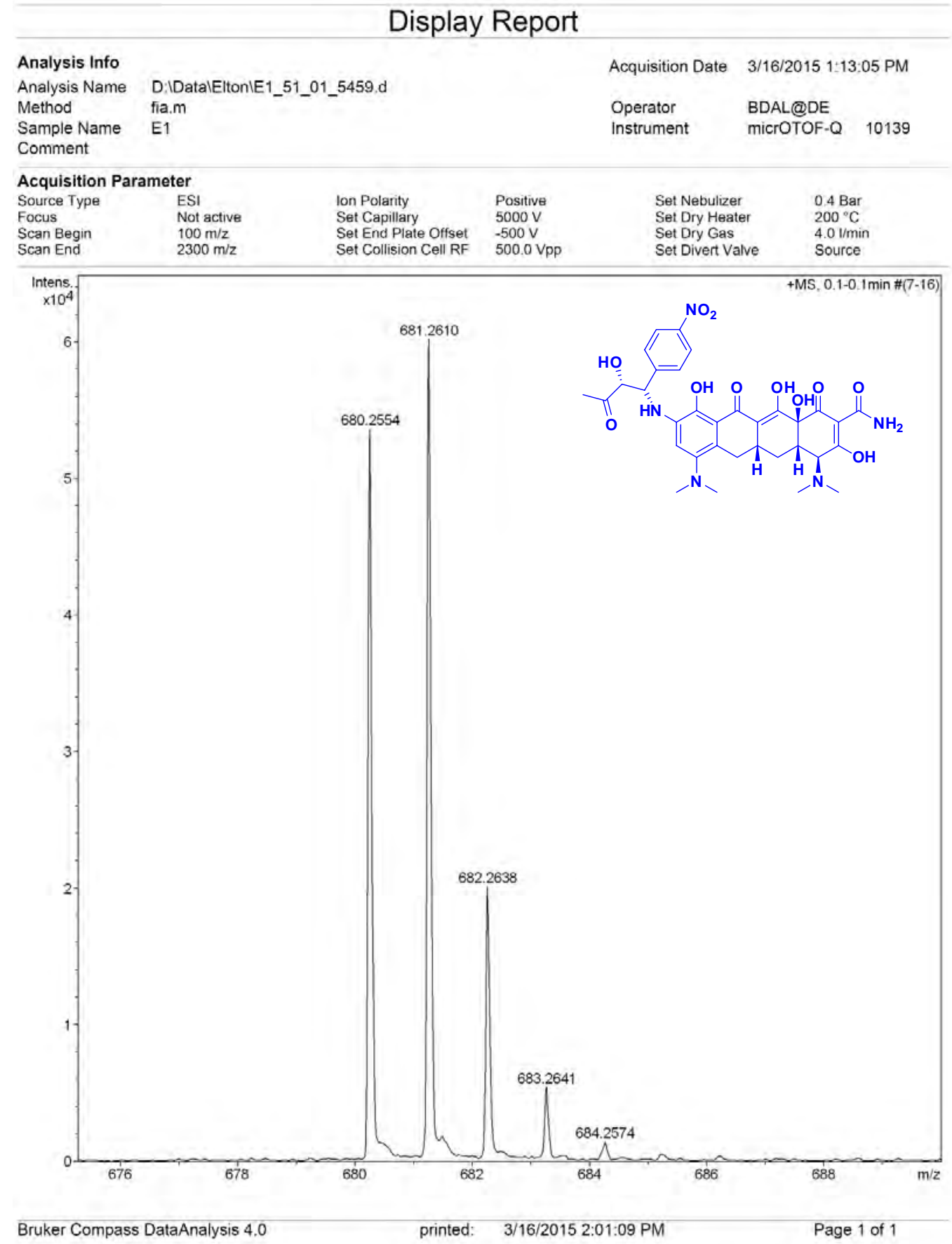


HRMS of (4S,4aS,5aR,12aS)-9-((1S,2R)-1-(4-butoxyphenyl)-2-hydroxy-3-oxobutylamino)4,7 bis(dimethylamino)-3,10,12,12a-tetrahydroxy-1,11-dioxo-1,4,4a,5,5a,6,11,12aoctahydrotetracene-2-carboxamide (2b)

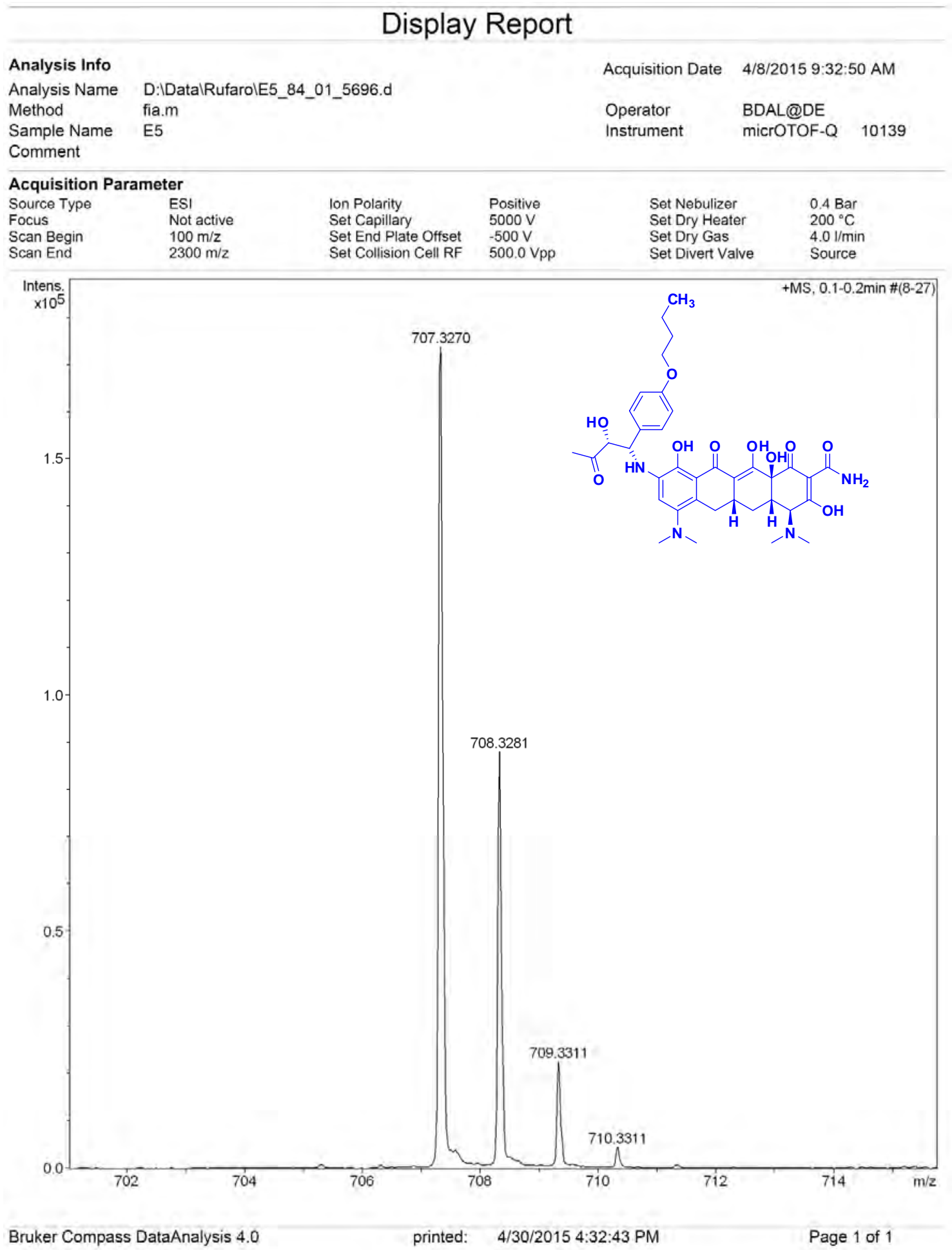


HRMS of (4S,4aS,5aR,12aS)-4,7-bis(dimethylamino)-3,10,12,12a-tetrahydroxy-9-((1S,2R)2-hydroxy-3-oxo-1-o-tolylbutylamino)-1,11-dioxo-1,4,4a,5,5a,6,11,12a-octahydrotetracene2-carboxamide (2c)

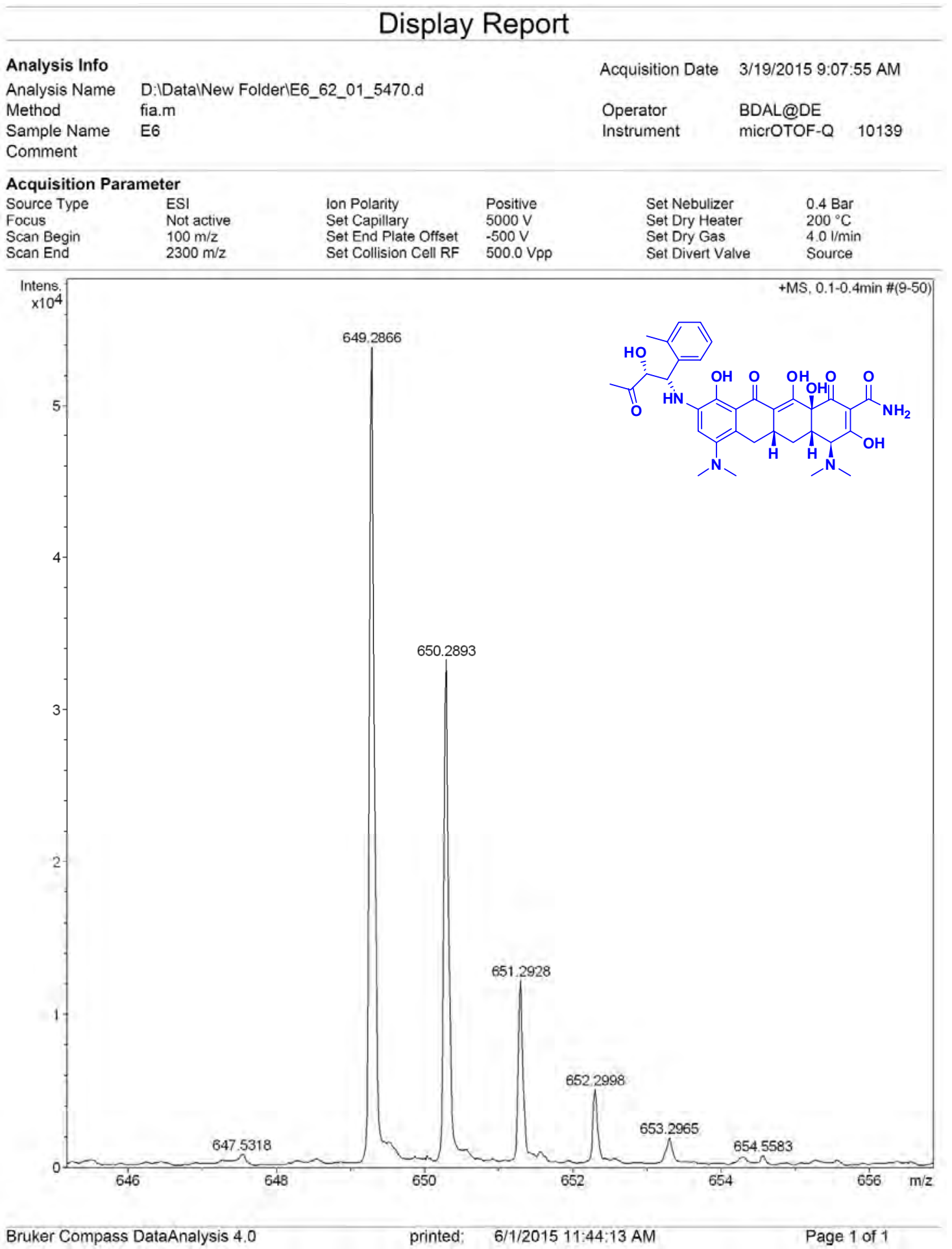


HRMS of (4S,4aS,5aR,12aS)-4,7-bis(dimethylamino)-9-((1S,2R)-1-(4-fluorophenyl)-2hydroxy-3-oxobutylamino)-3,10,12,12a-tetrahydroxy-1,11-dioxo-1,4,4a,5,5a,6,11,12aoctahydrotetracene-2-carboxamide (2d)

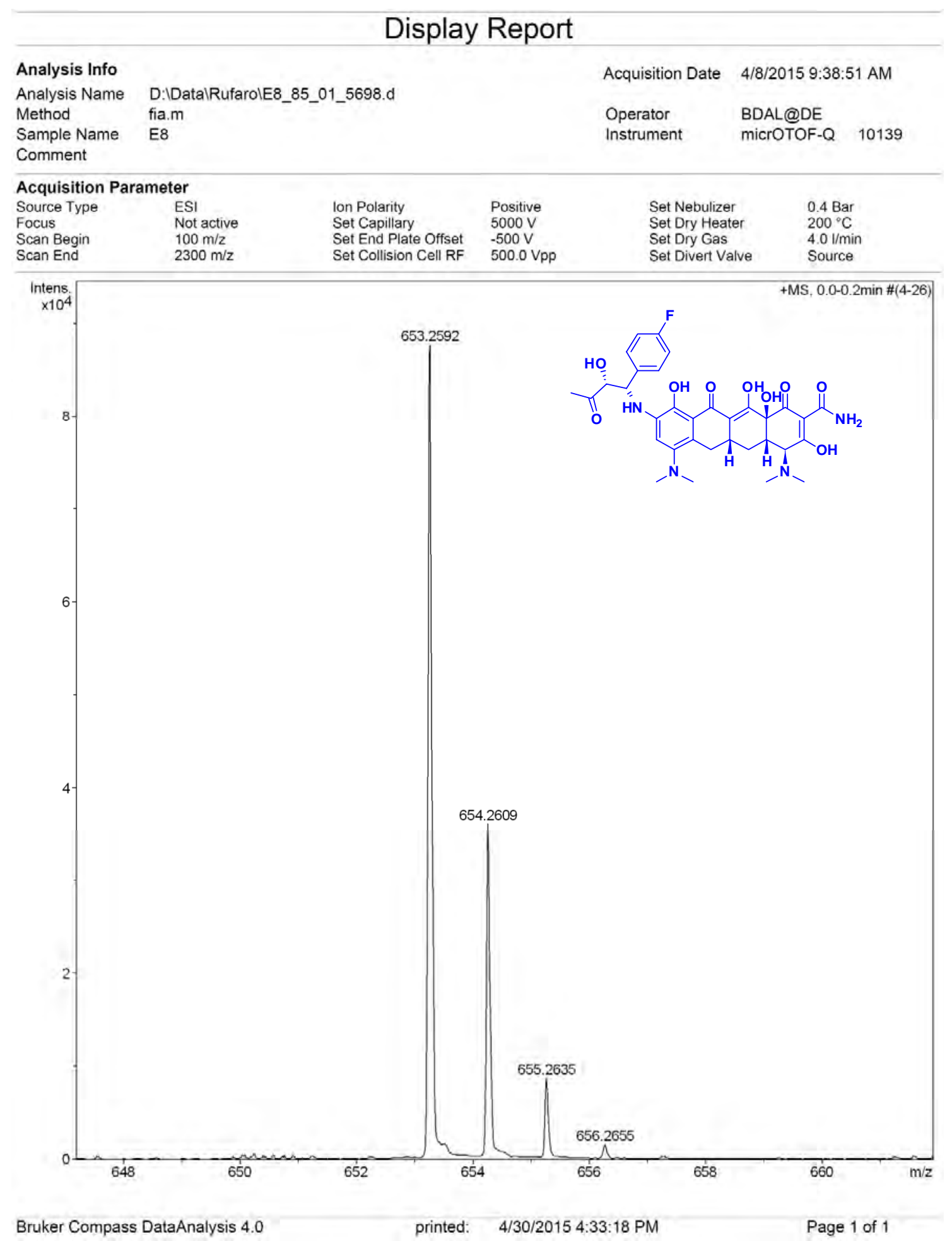


HRMS of (4S,4aS,5aR,12aS)-4,7-bis(dimethylamino)-3,10,12,12a-tetrahydroxy-9-((1S,2R)-2hydroxy-1-(naphthalen-2-yl)-3-oxobutylamino)-1,11-dioxo-1,4,4a,5,5a,6,11,12aoctahydrotetracene-2-carboxamide (2e)

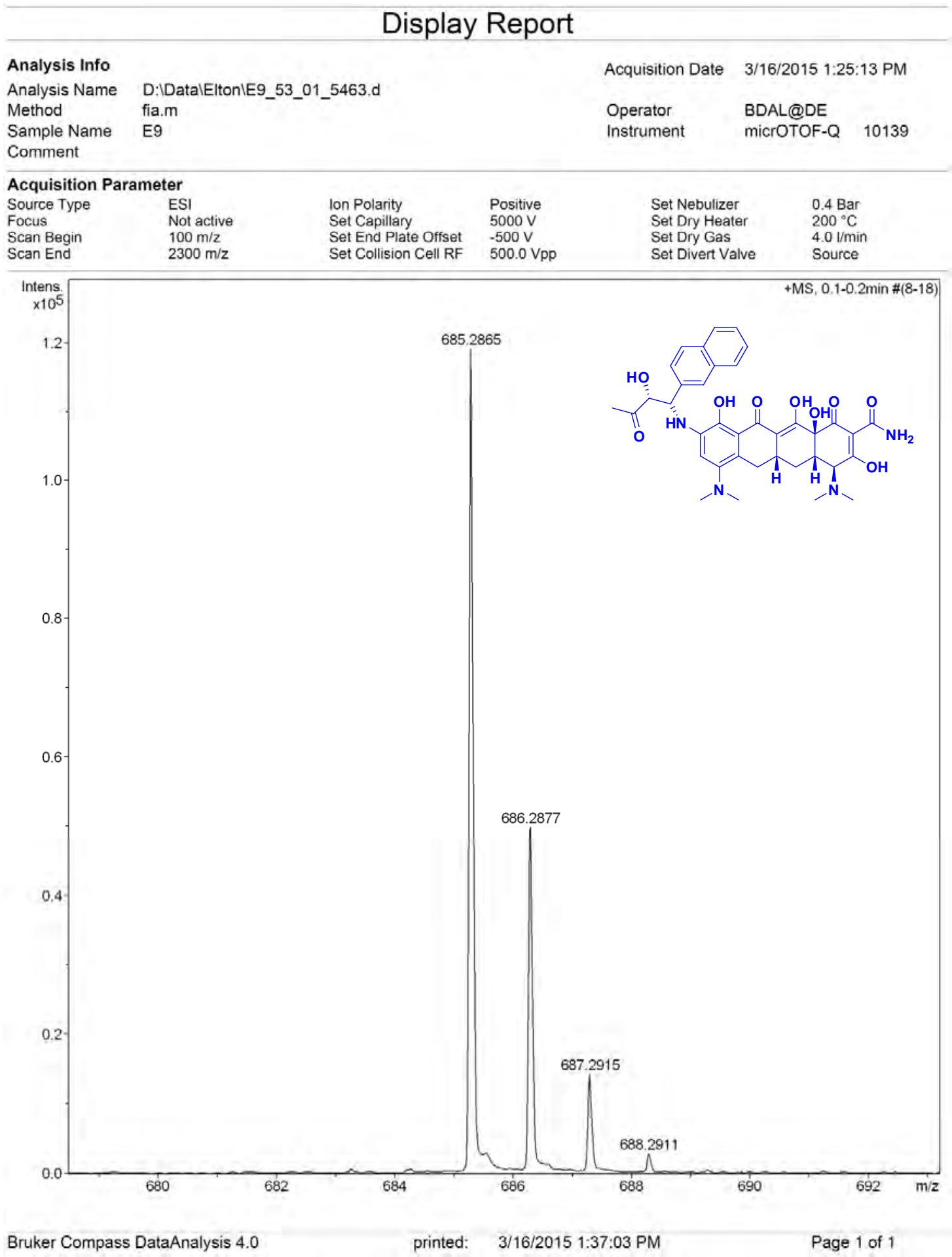


HRMS of (4S,4aS,5aR,12aS)-4,7-bis(dimethylamino)-3,10,12,12a-tetrahydroxy-9-((1R,2R)-

2-hydroxy-1-(5-nitrofuran-2-yl)-3-oxobutylamino)-1,11-dioxo-1,4,4a,5,5a,6,11,12

octahydrotetracene-2-carboxamide (2f)

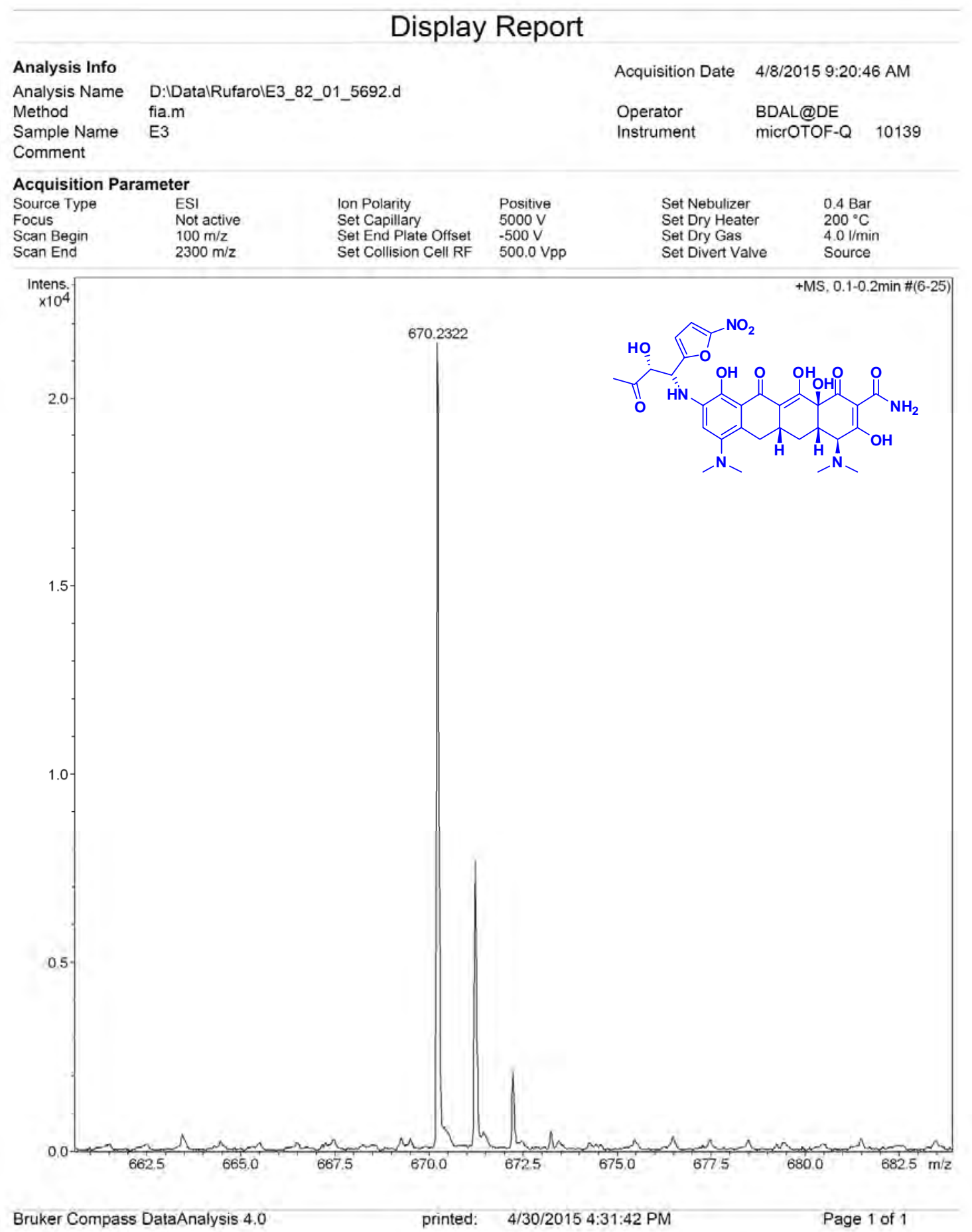


HRMS of (4S,4aS,5aR,12aS)-4,7-bis(dimethylamino)-9-((1R,2R)-1-(furan-2-yl)-2-hydroxy-

3-oxobutylamino)-3,10,12,12a-tetrahydroxy-1,11-dioxo-1,4,4a,5,5a,6,11,12aoctahydrotetracene-2-carboxamide (2g)

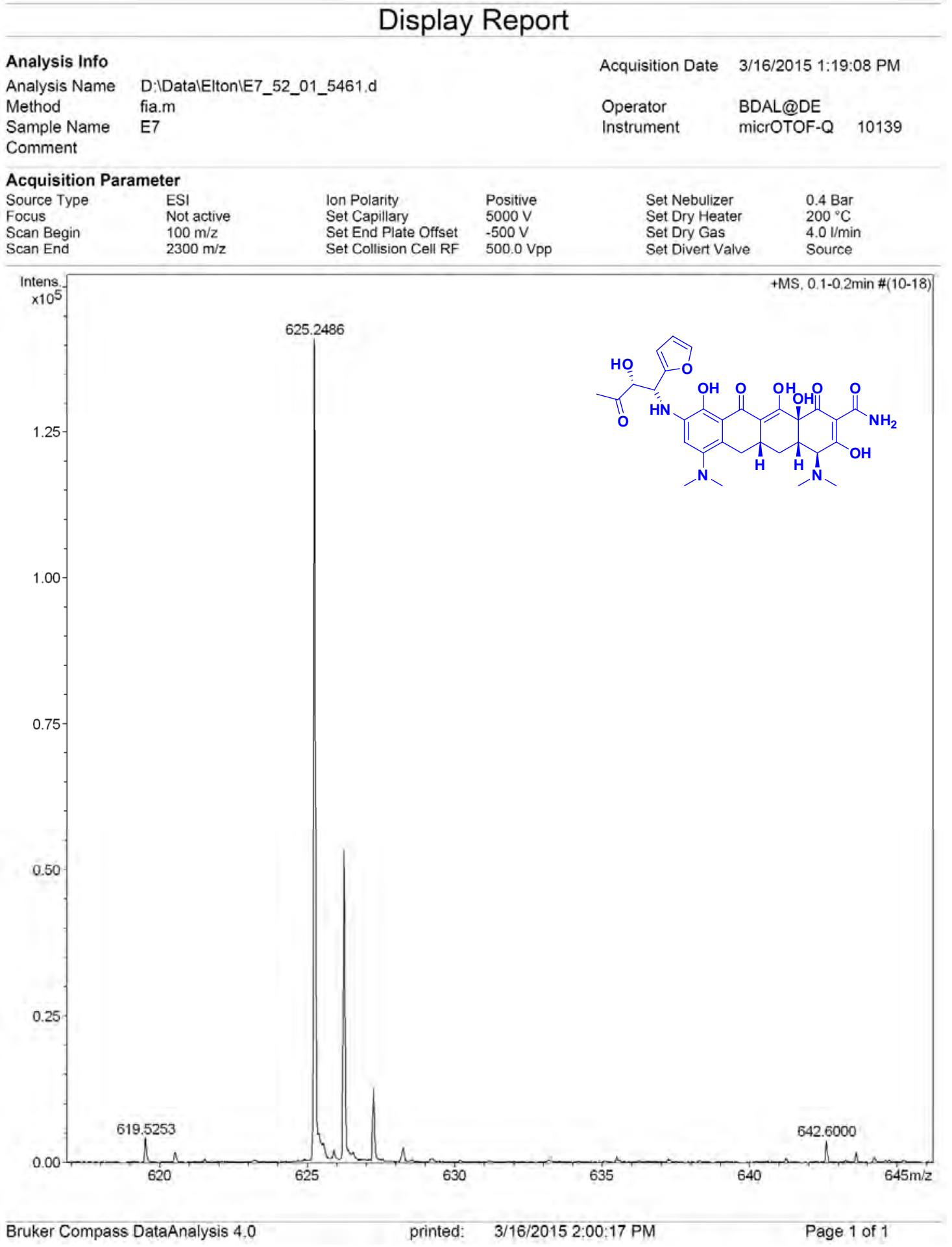


HRMS of (4S,4aS,5aR,12aS)-9-(1-(5-bromofuran-2-yl)-2-hydroxy-3-oxobutylamino)-4,7-bis (dimethylamino)-3,10,12,12a-tetrahydroxy-1,11-dioxo-1,4,4a,5,5a,6,11,12aoctahydrotetracene-2-carboxamide $(2 \mathrm{~h})$

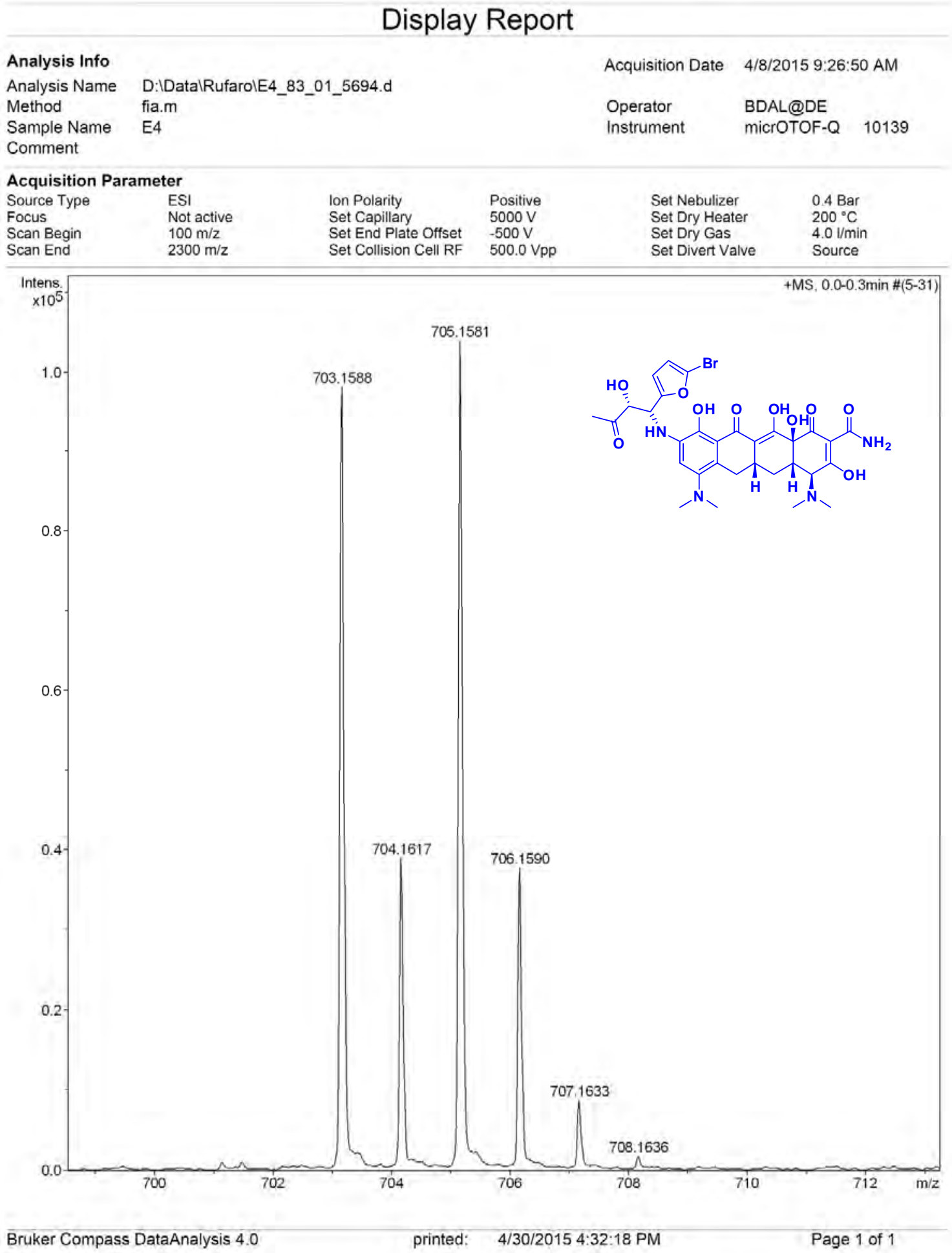




\section{Copies of LC-MS spectra for products}

LC-MS of (4S,4aS,5aR,12aS)-4,7-bis(dimethylamino)-3,10,12,12a-tetrahydroxy-9-(2hydroxy-3-oxo-1-phenylbutylamino)-1,11-dioxo-1,4,4a,5,5a,6,11,12a-octahydrotetracene-2carboxamide (1a)

\section{==== Shimadzu LabSolutions Multi-Chromatogram ====}

mAU
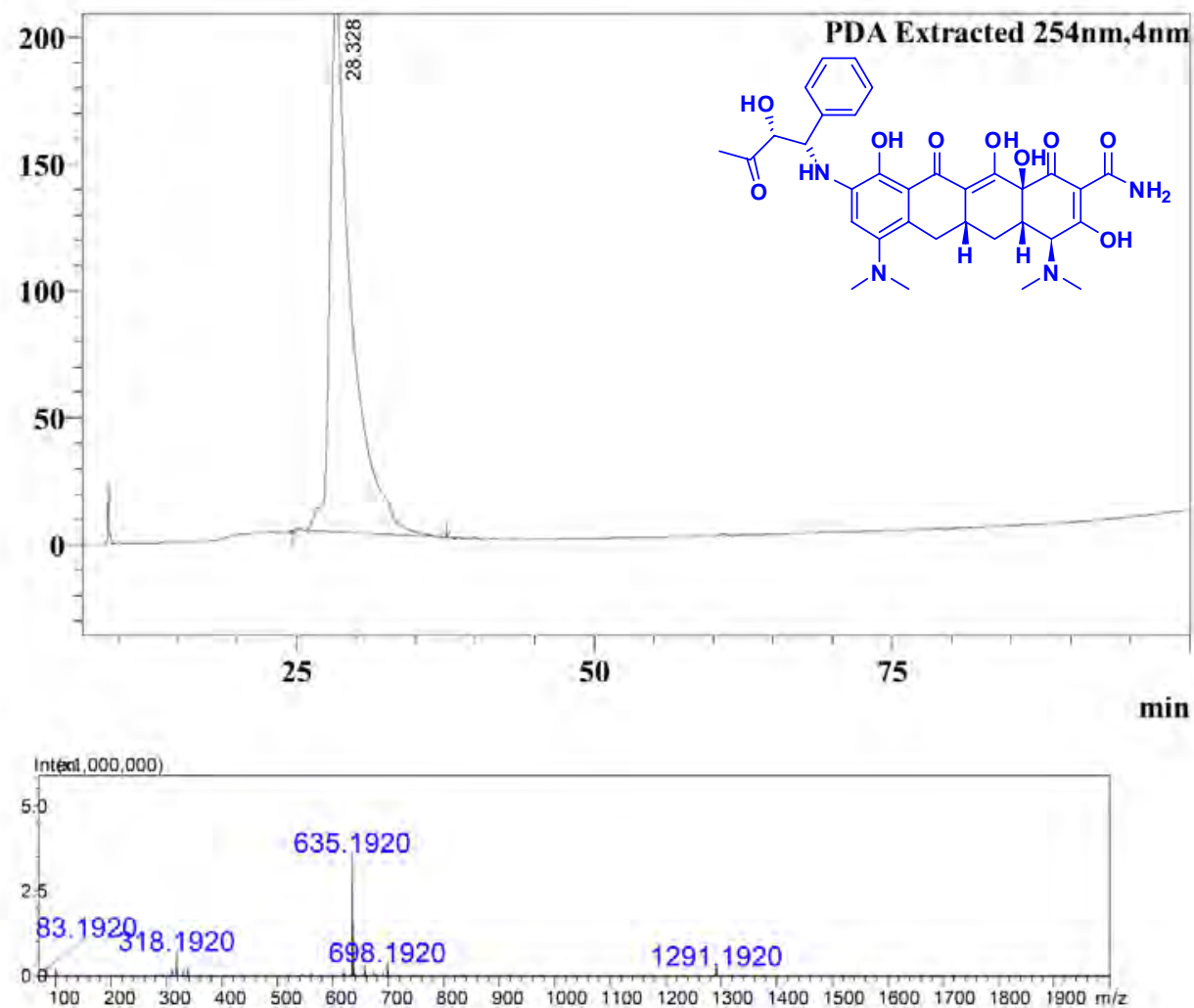
LC-MS of (4S,4aS,5aR,12aS)-4,7-bis(dimethylamino)-3,10,12,12a-tetrahydroxy-9-(2methyl-3-oxo-1-phenylbutylamino)-1,11-dioxo-1,4,4a,5,5a,6,11,12a-octahydrotetracene-2carboxamide (1b)

14/10/2015 16:06:09 Page $1 / 5$

==== Shimadzu LabSolutions Multi-Chromatogram ==== $\mathrm{mAU}$
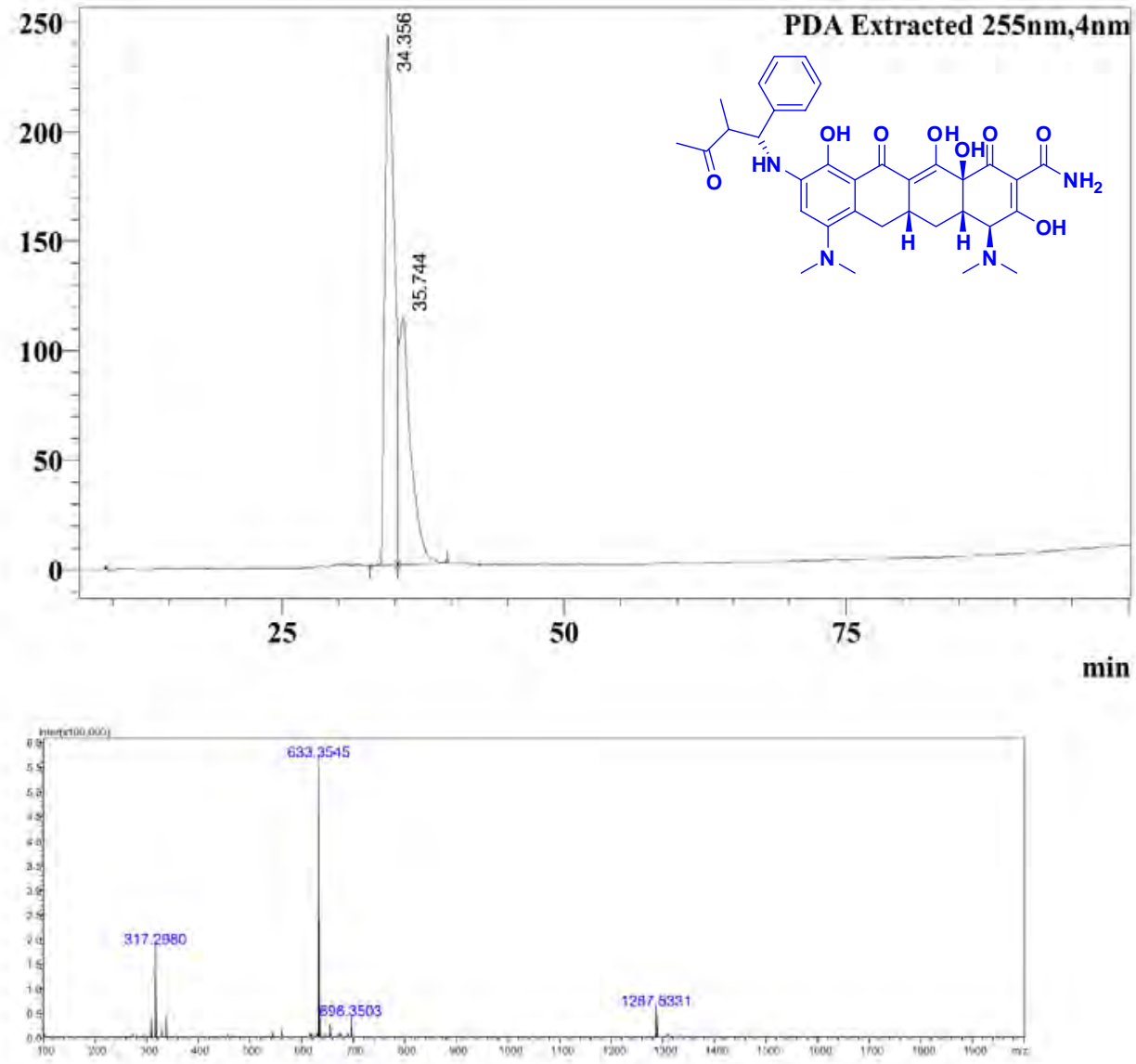
LC-MS of (4S,4aS,5aR,12aS)-4,7-bis(dimethylamino)-3,10,12,12a-tetrahydroxy-1,11-dioxo9-(3-oxo-1-phenylbutylamino)-1,4,4a,5,5a,6,11,12a-octahydrotetracene-2-carboxamide (1c)

17/10/2015 18:11:31 Page 1/5
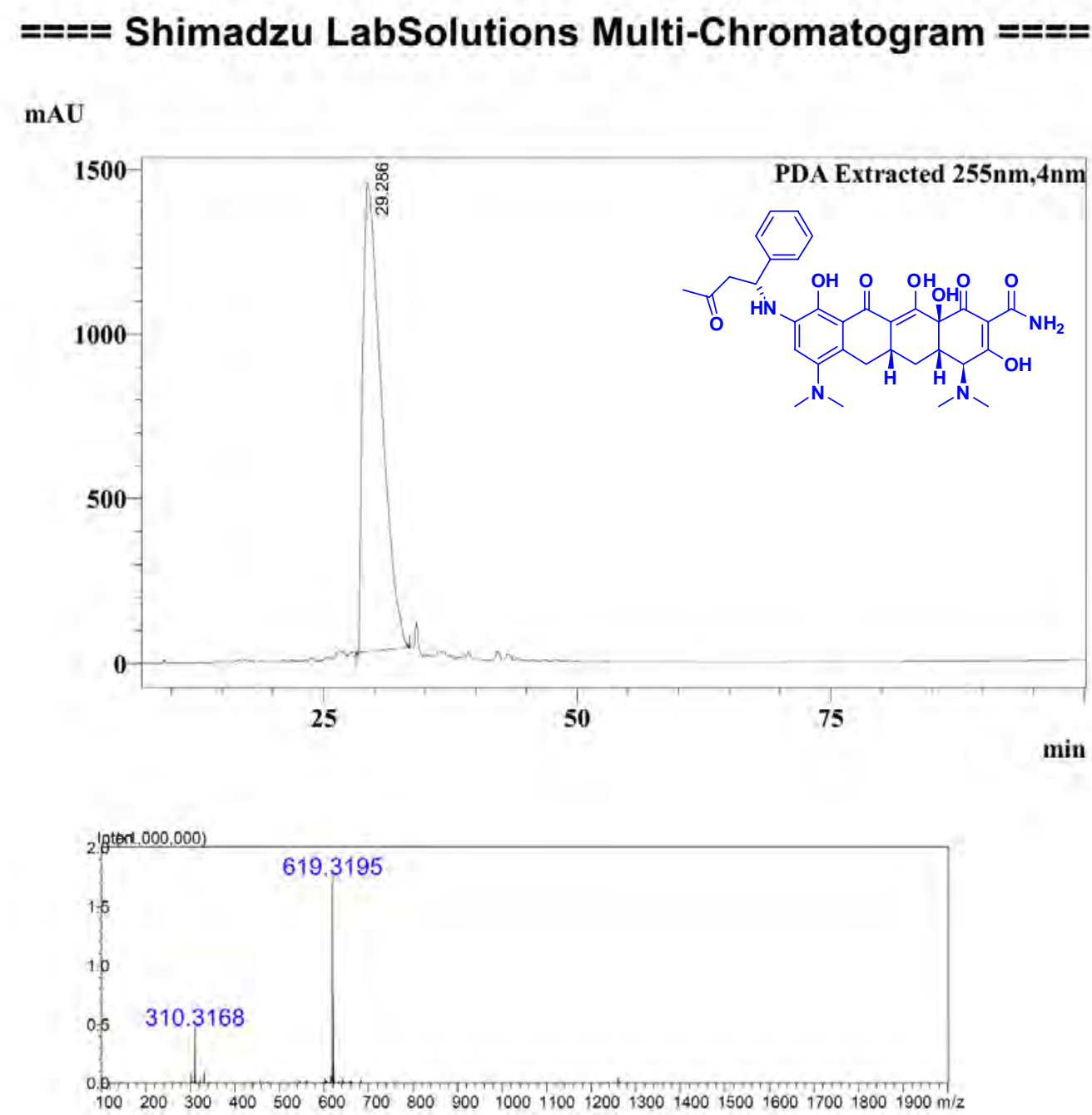
LC-MS of (4S,4aS,5aR,12aS)-4,7-bis(dimethylamino)-3,10,12,12a-tetrahydroxy-1,11-dioxo9-((1R)-(2-oxocyclohexyl)(phenyl)methylamino)-1,4,4a,5,5a,6,11,12a-octahydrotetracene-2carboxamide (1d)

\section{=ニ== Shimadzu LabSolutions Multi-Chromatogram ==== mAU}
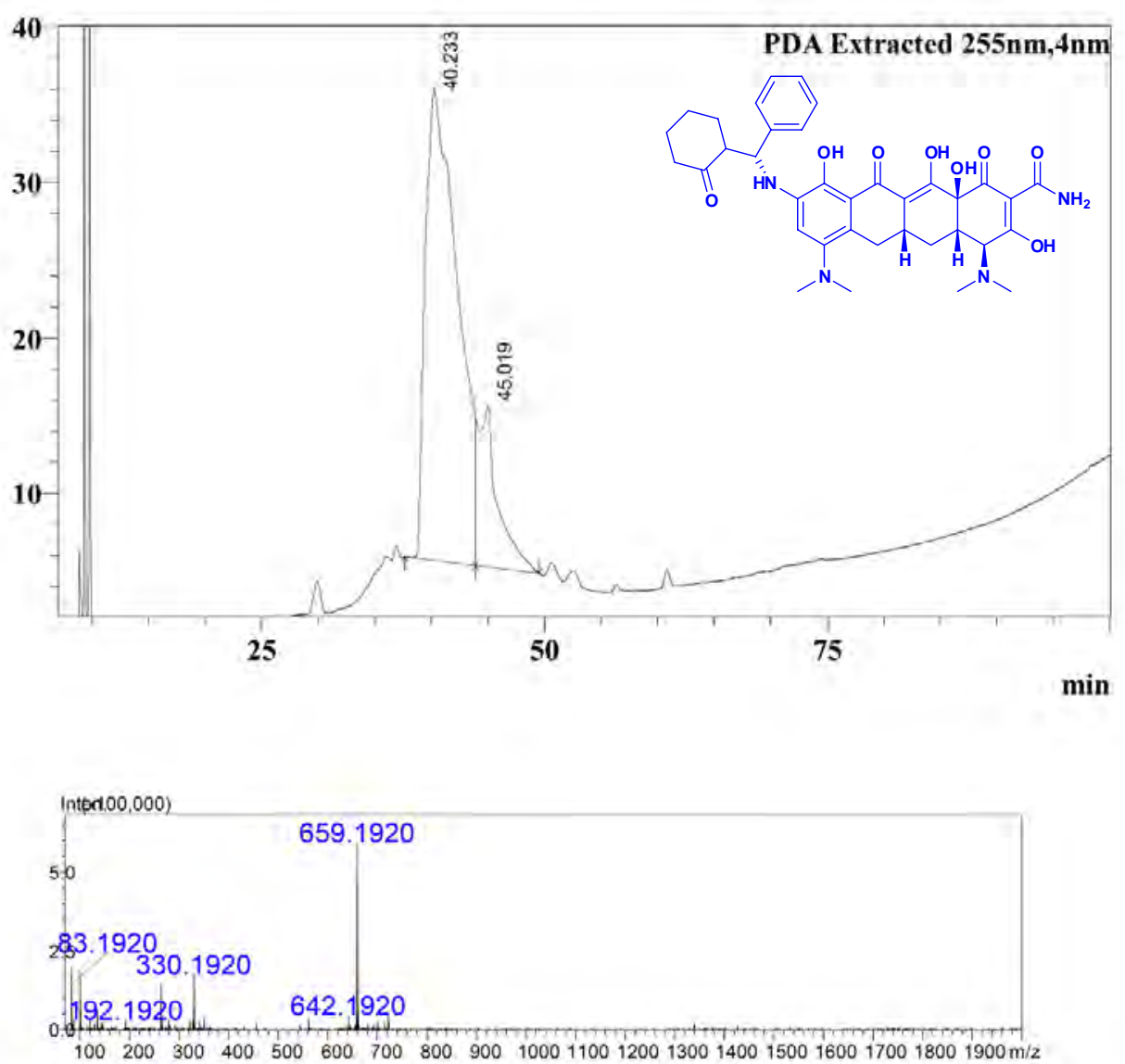
LC-MS of (4S,4aS,5aR,12aS)-4,7-bis(dimethylamino)-3,10,12,12a-tetrahydroxy-9-((1S,2R)2-hydroxy-1-(4-nitrophenyl)-3-oxobutylamino)-1,11-dioxo-1,4,4a,5,5a,6,11,12aoctahydrotetracene-2-carboxamide (2a)

\section{==== Shimadzu LabSolutions Multi-Chromatogram ====} mAU
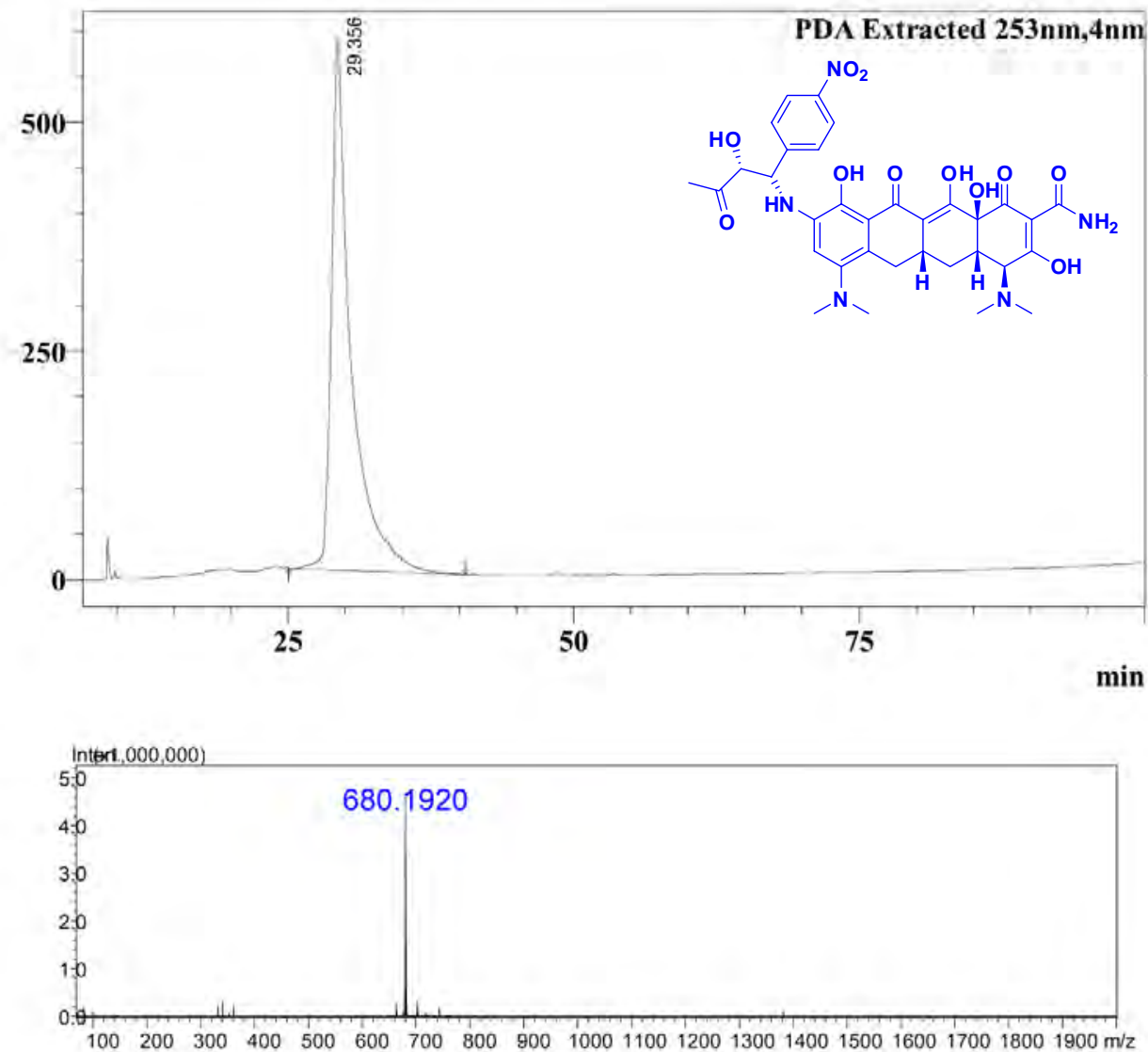
LC-MS of (4S,4aS,5aR,12aS)-9-((1S,2R)-1-(4-butoxyphenyl)-2-hydroxy-3-oxobutylamino)4,7 bis(dimethylamino)-3,10,12,12a-tetrahydroxy-1,11-dioxo-1,4,4a,5,5a,6,11,12aoctahydrotetracene-2-carboxamide (2b)

14/10/2015 15:29:14 Page 1
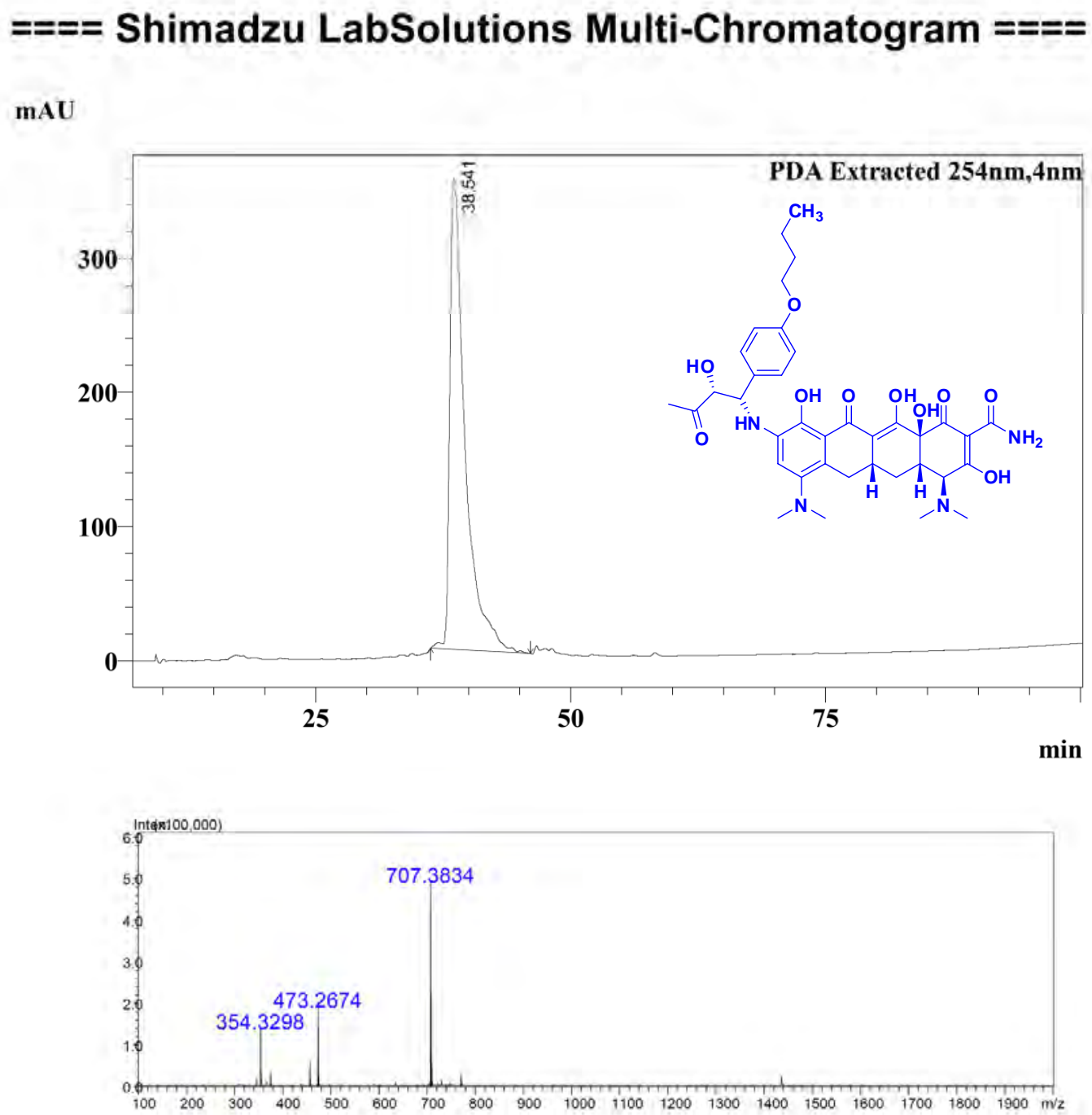
LC-MS of (4S,4aS,5aR,12aS)-4,7-bis(dimethylamino)-3,10,12,12a-tetrahydroxy-9-((1S,2R)2-hydroxy-3-oxo-1-o-tolylbutylamino)-1,11-dioxo-1,4,4a,5,5a,6,11,12a-octahydrotetracene2-carboxamide (2c)
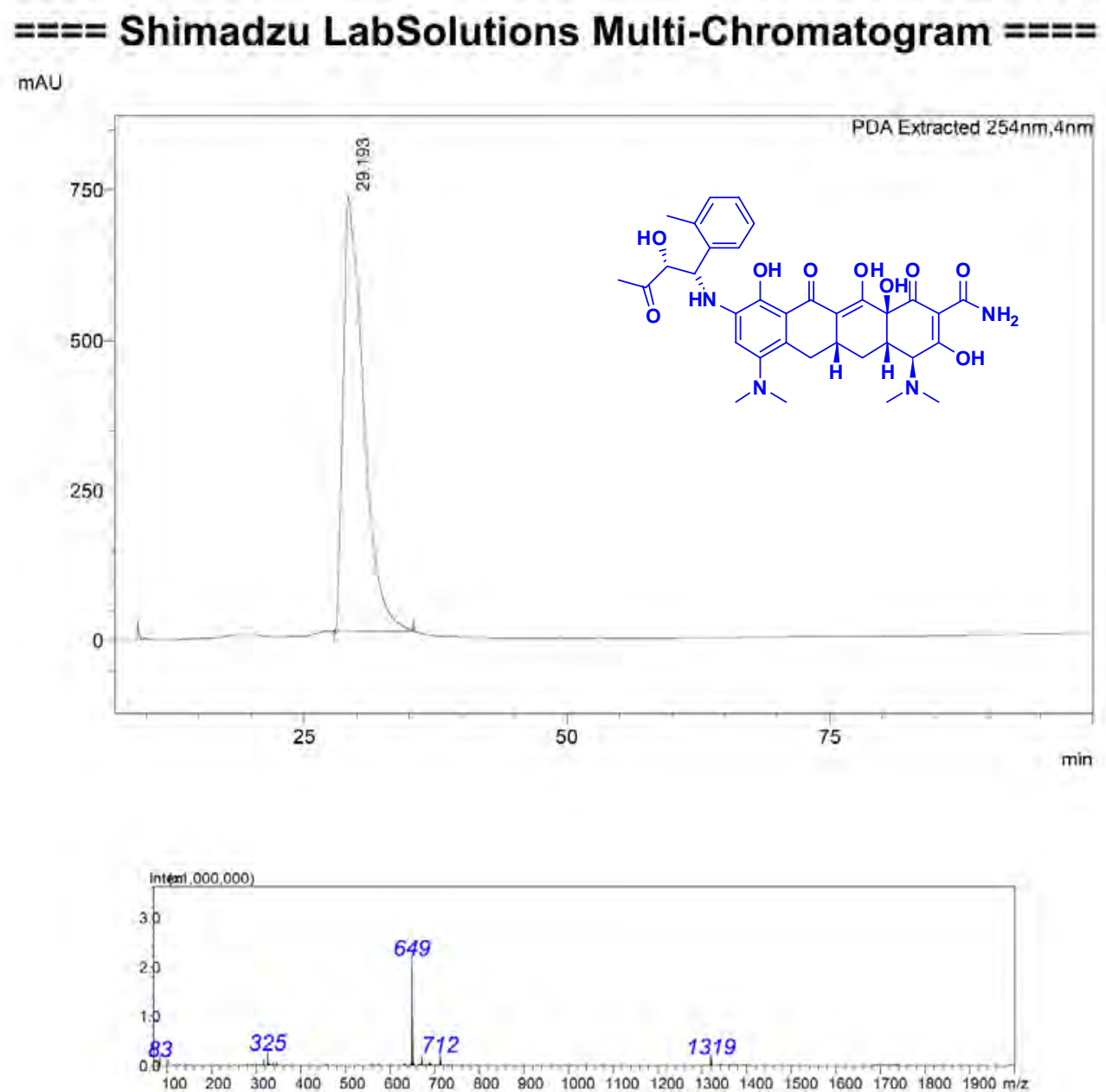
LC-MS SPECTRUM of (4S,4aS,5aR,12aS)-4,7-bis(dimethylamino)-9-((1S,2R)-1-(4fluorophenyl)-2-hydroxy-3-oxobutylamino)-3,10,12,12a-tetrahydroxy-1,11-dioxo1,4,4a,5,5a,6,11,12a-octahydrotetracene-2-carboxamide (2d)

==== Shimadzu LabSolutions Multi-Chromatogram ====

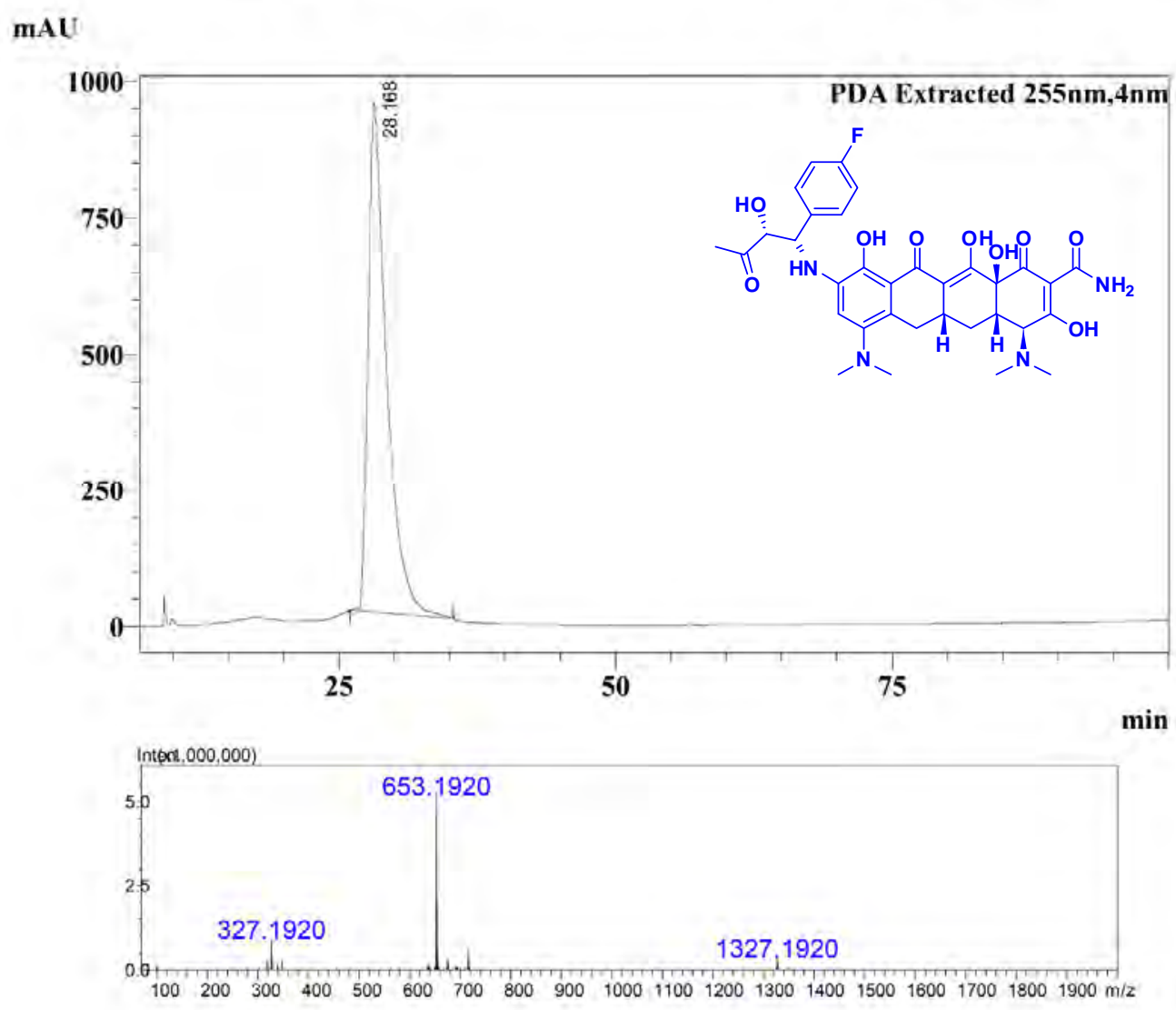


LC-MS of (4S,4aS,5aR,12aS)-4,7-bis(dimethylamino)-3,10,12,12a-tetrahydroxy-9-((1S,2R)2-hydroxy-1-(naphthalen-2-yl)-3-oxobutylamino)-1,11-dioxo-1,4,4a,5,5a,6,11,12aoctahydrotetracene-2-carboxamide (2e)

==== Shimadzu LabSolutions Multi-Chromatogram ==== mAU
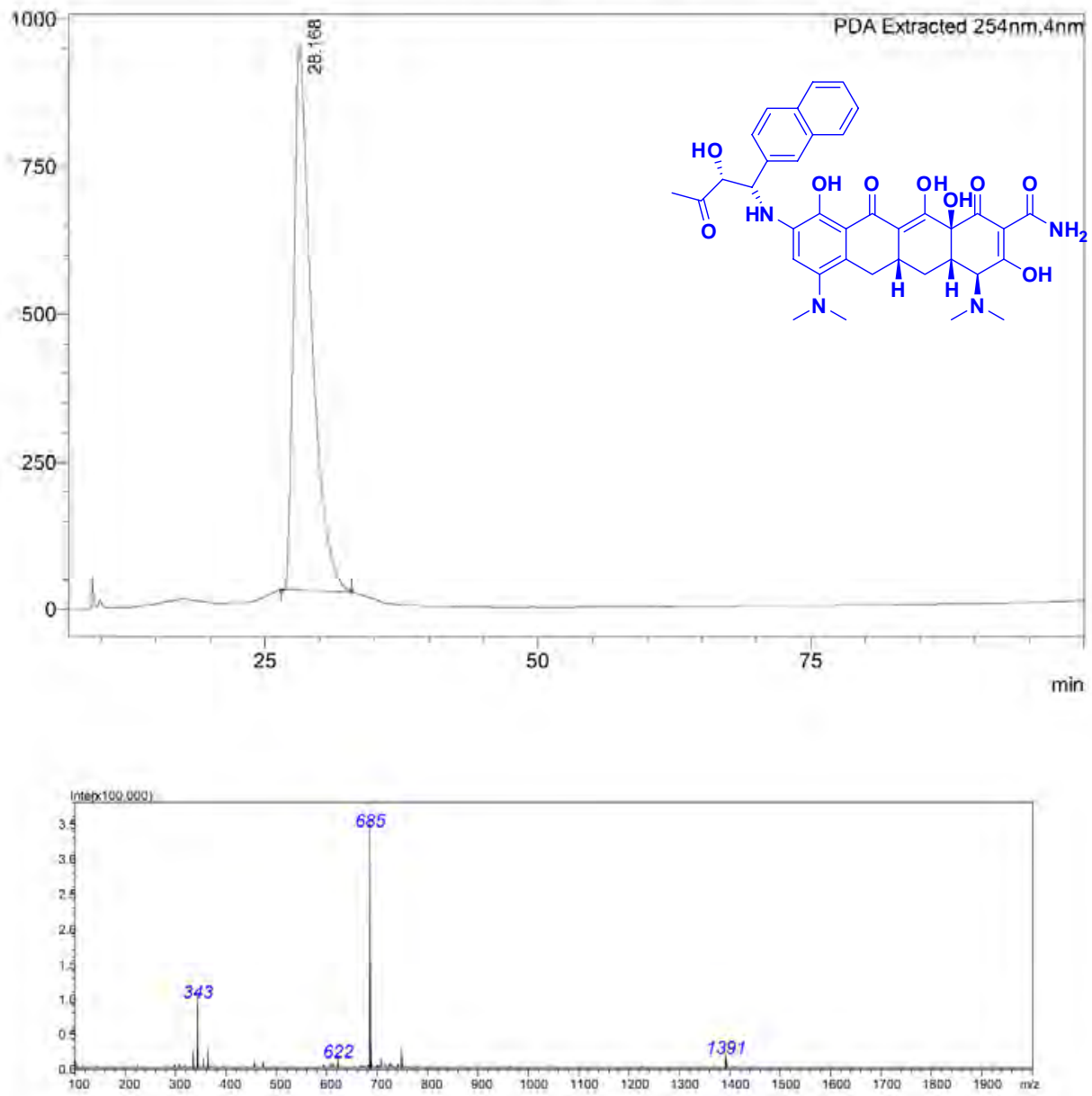
LC-MS of (4S,4aS,5aR,12aS)-4,7-bis(dimethylamino)-3,10,12,12a-tetrahydroxy-9-((1R,2R)2-hydroxy-1-(5-nitrofuran-2-yl)-3-oxobutylamino)-1,11-dioxo-1,4,4a,5,5a,6,11,12 octahydrotetracene-2-carboxamide (2f)

\section{=ニ== Shimadzu LabSolutions Multi-Chromatogram ==== mAU}
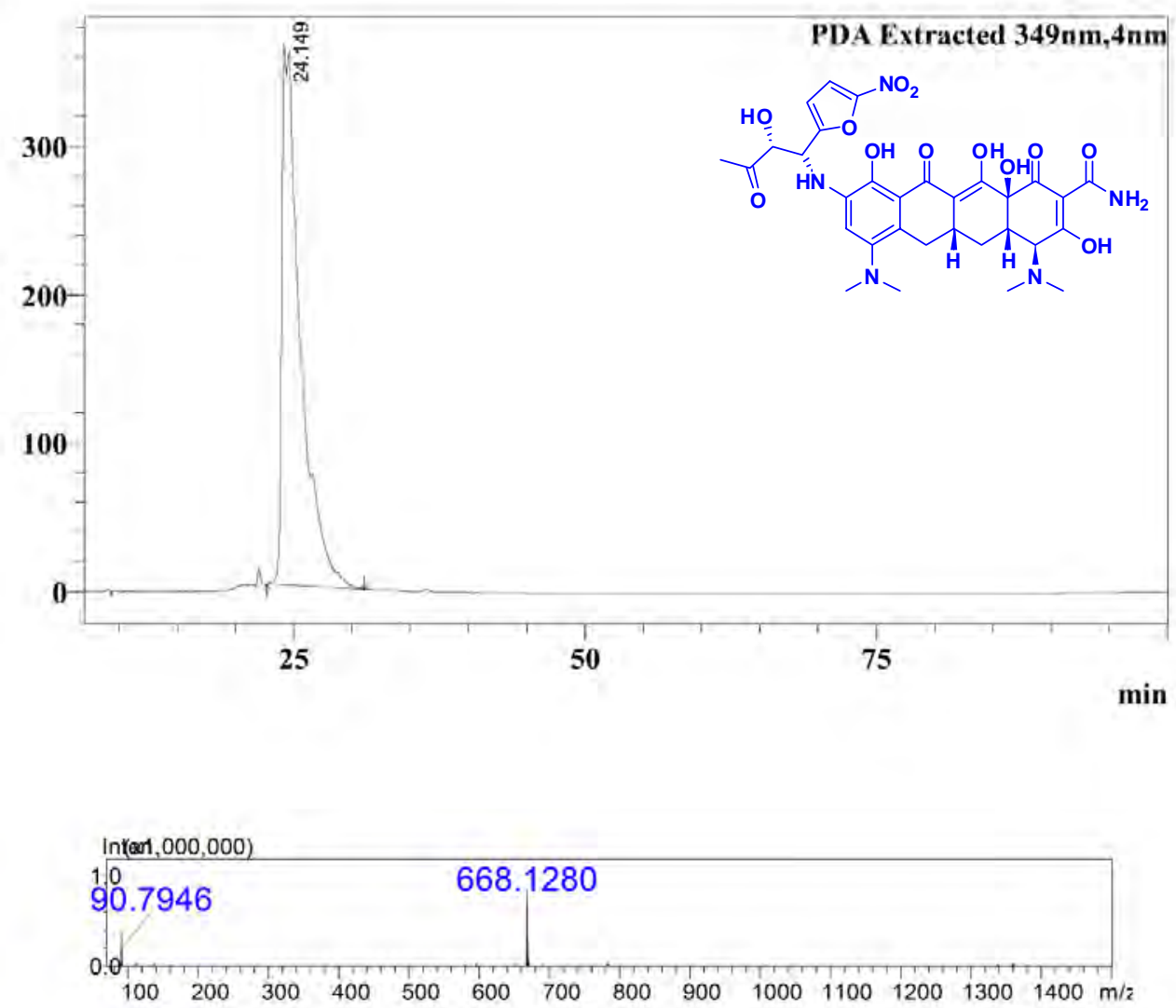
LC-MS of (4S,4aS,5aR,12aS)-4,7-bis(dimethylamino)-9-((1R,2R)-1-(furan-2-yl)-2-hydroxy3-oxobutylamino)-3,10,12,12a-tetrahydroxy-1,11-dioxo-1,4,4a,5,5a,6,11,12aoctahydrotetracene-2-carboxamide (2g)
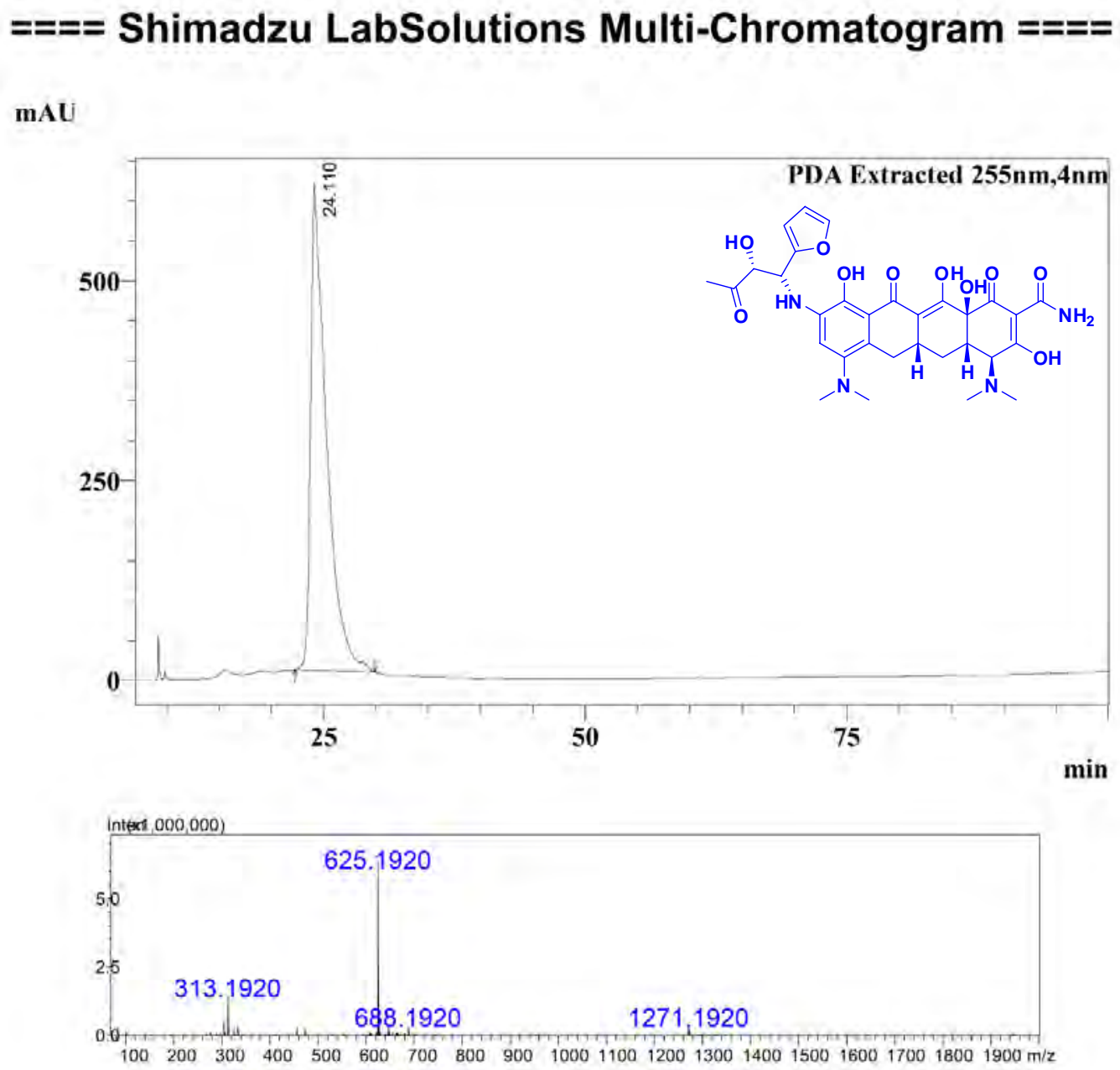
LC-MS of (4S,4aS,5aR,12aS)-9-(1-(5-bromofuran-2-yl)-2-hydroxy-3-oxobutylamino)-4,7bis (dimethylamino)-3,10,12,12a-tetrahydroxy-1,11-dioxo-1,4,4a,5,5a,6,11,12aoctahydrotetracene-2-carboxamide (2h)

14/10/2015 15:15:01 Page 1

==== Shimadzu LabSolutions Multi-Chromatogram ==== mAU
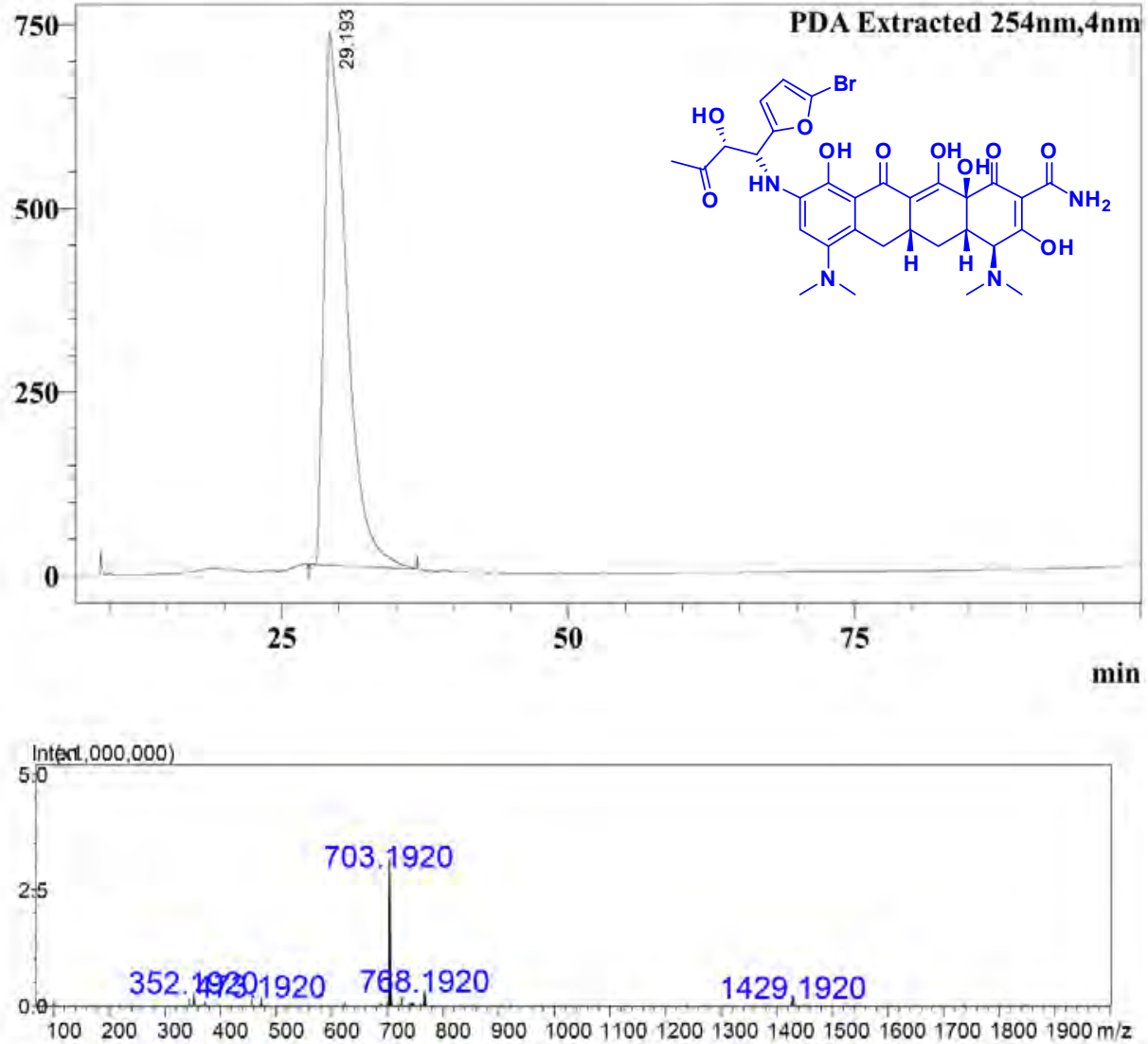


\section{Copies of NMR spectra for products}

${ }^{1} \mathrm{H}$ NMR of (4S,4aS,5aR,12aS)-4,7-bis(dimethylamino)-3,10,12,12a-tetrahydroxy-9-(2-hydroxy-3-oxo-1-phenylbutylamino)1,11-dioxo-1,4,4a,5,5a,6,11,12a-octahydrotetracene-2-carboxamide (1a)

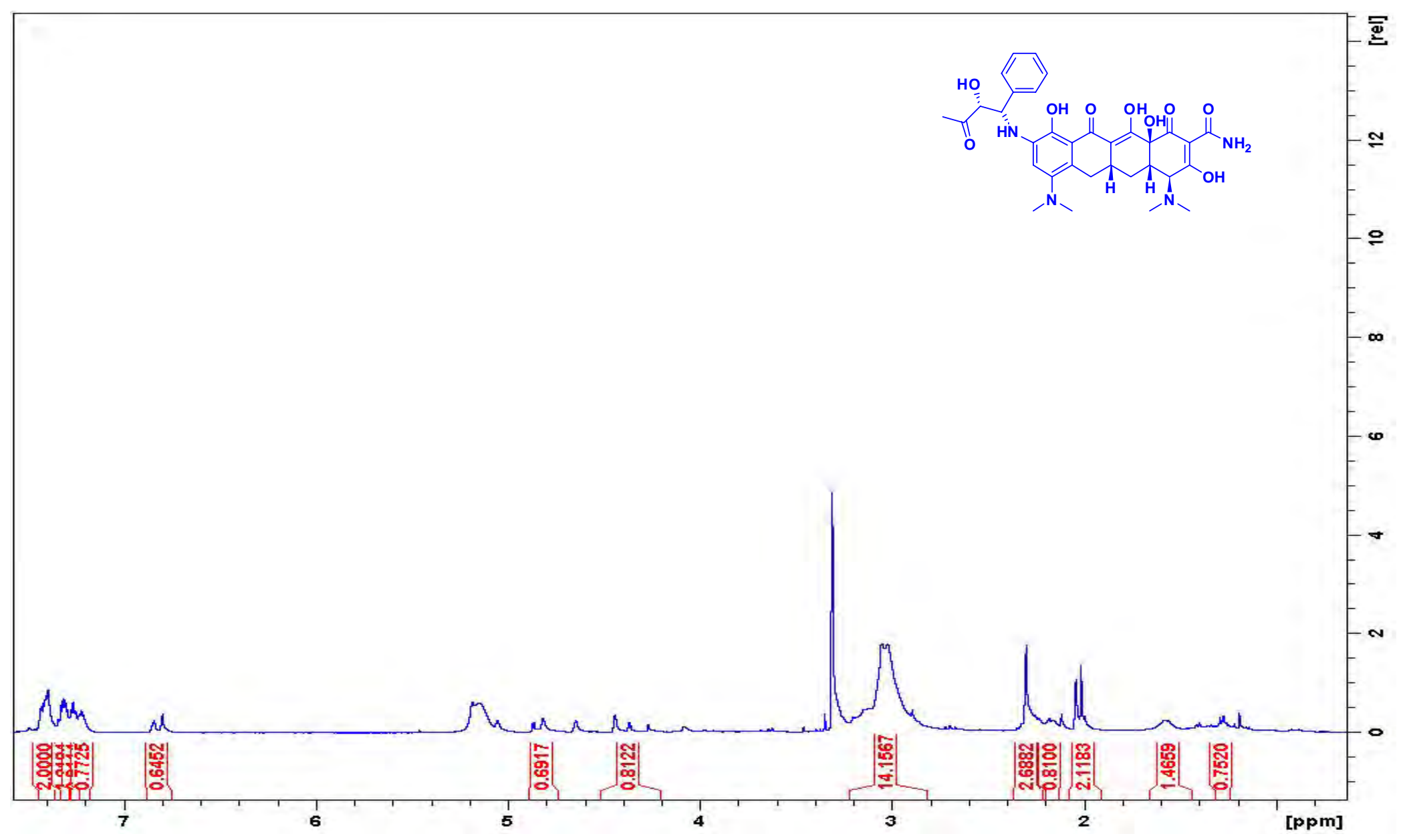


${ }^{1} \mathrm{H}$ NMR of (4S,4aS,5aR,12aS)-4,7-bis(dimethylamino)-3,10,12,12a-tetrahydroxy-9-(2-methyl-3-oxo-1-phenylbutylamino)-1,11dioxo-1,4,4a,5,5a,6,11,12a-octahydrotetracene-2-carboxamide (1b)

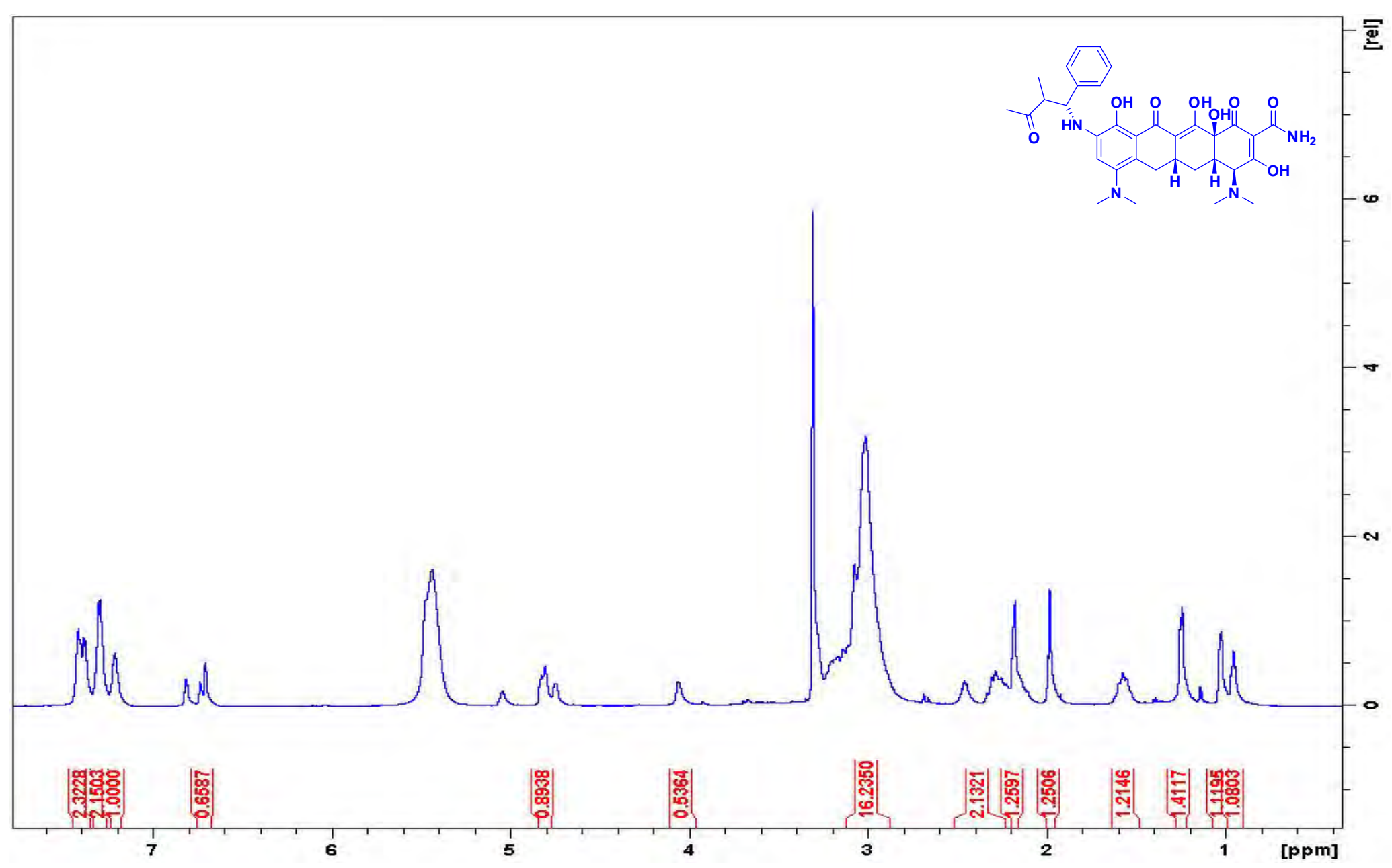


${ }^{1}$ H NMR of (4S,4aS,5aR,12aS)-4,7-bis(dimethylamino)-3,10,12,12a-tetrahydroxy-1,11-dioxo-9-(3-oxo-1-phenylbutylamino)1,4,4a,5,5a,6,11,12a-octahydrotetracene-2-carboxamide (1c)

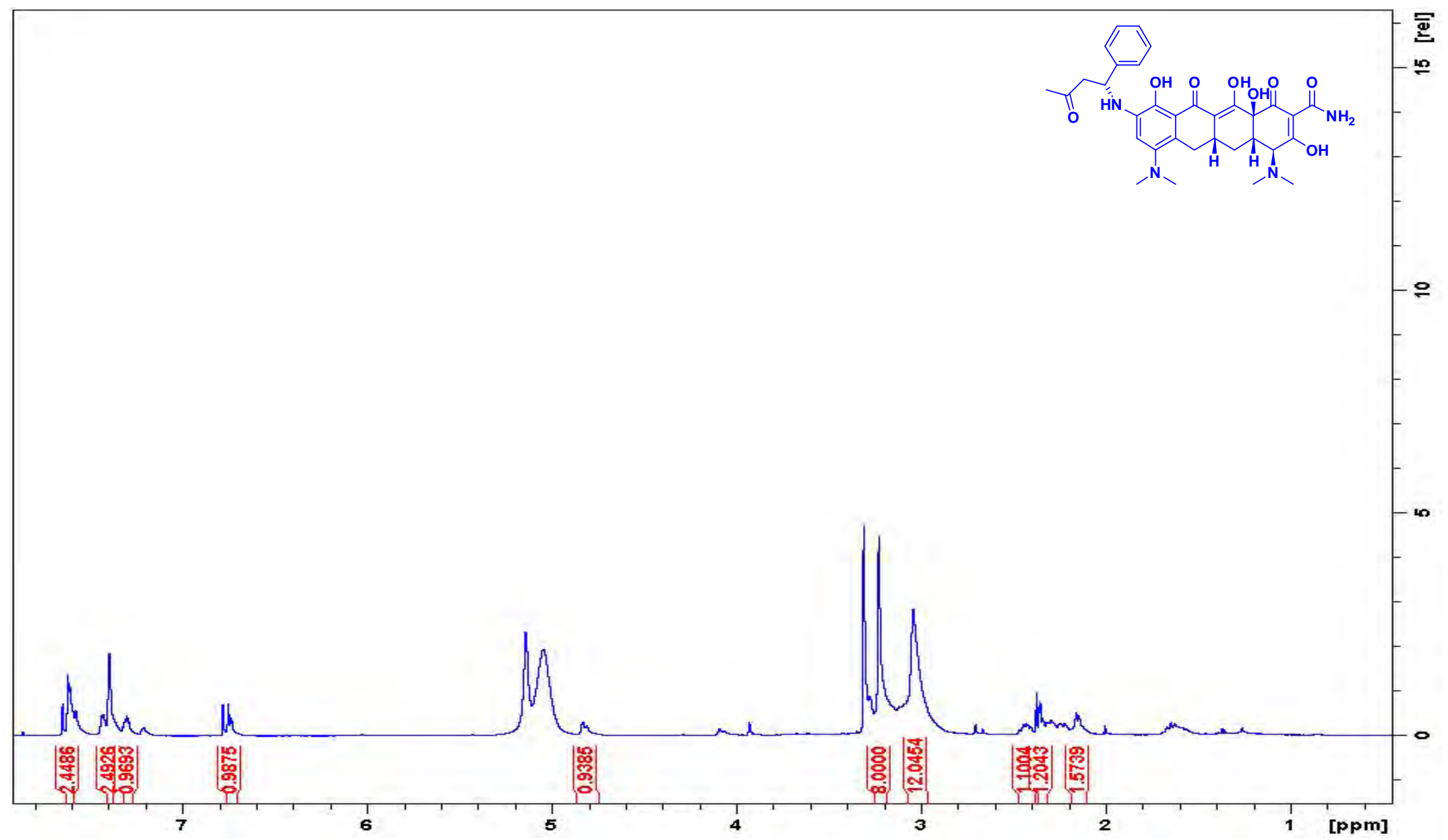


${ }^{1} \mathrm{H}$ NMR of (4S,4aS,5aR,12aS)-4,7-bis(dimethylamino)-3,10,12,12a-tetrahydroxy-1,11-dioxo-9-((1R)-(2oxocyclohexyl)(phenyl)methylamino)-1,4,4a,5,5a,6,11,12a-octahydrotetracene-2-carboxamide (1d)

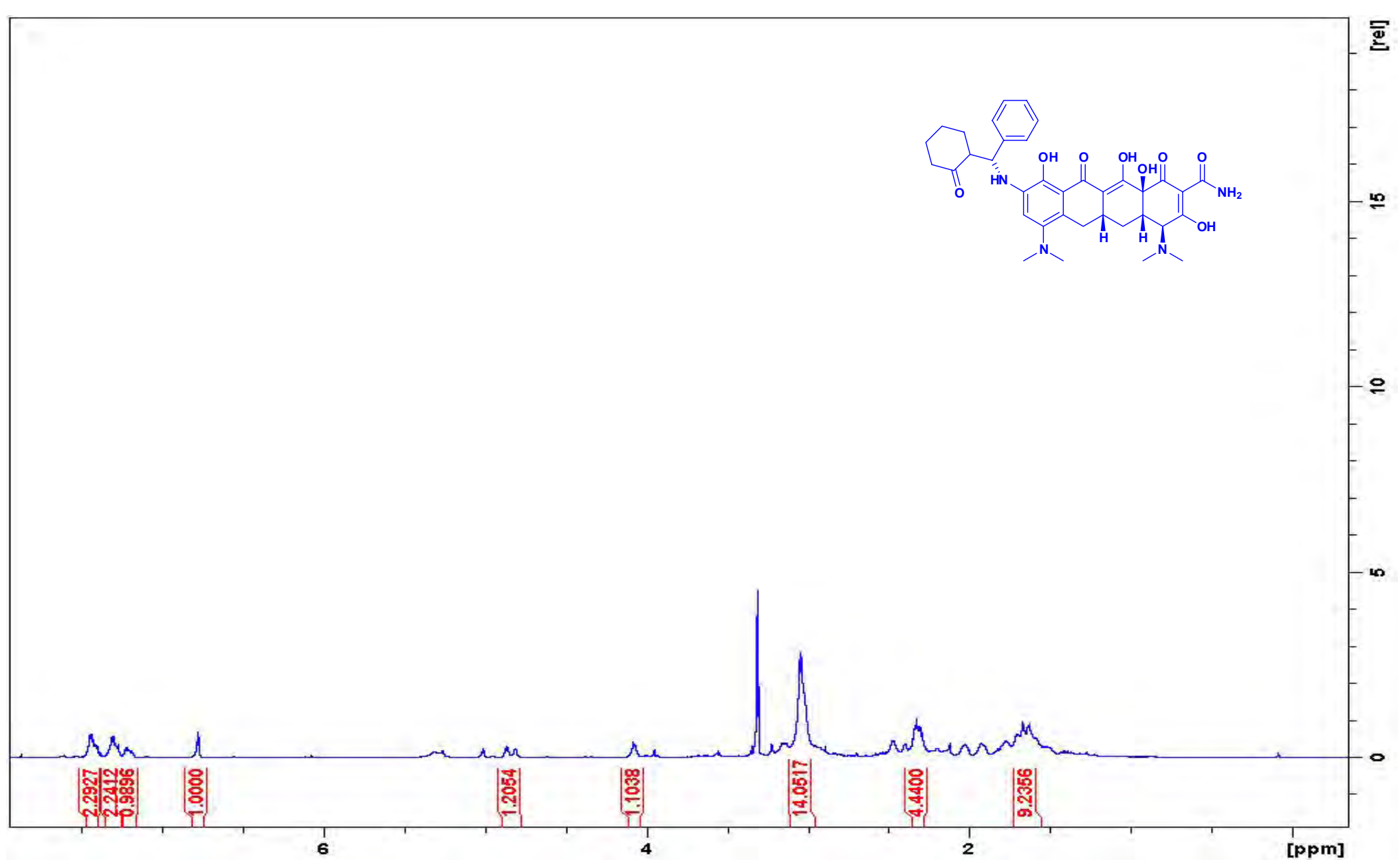


${ }^{1}$ H NMR of (4S,4aS,5aR,12aS)-4,7-bis(dimethylamino)-3,10,12,12a-tetrahydroxy-9-((1S,2R)-2-hydroxy-1-(4-nitrophenyl)-3oxobutylamino)-1,11-dioxo-1,4,4a,5,5a,6,11,12a-octahydrotetracene-2-carboxamide (2a)

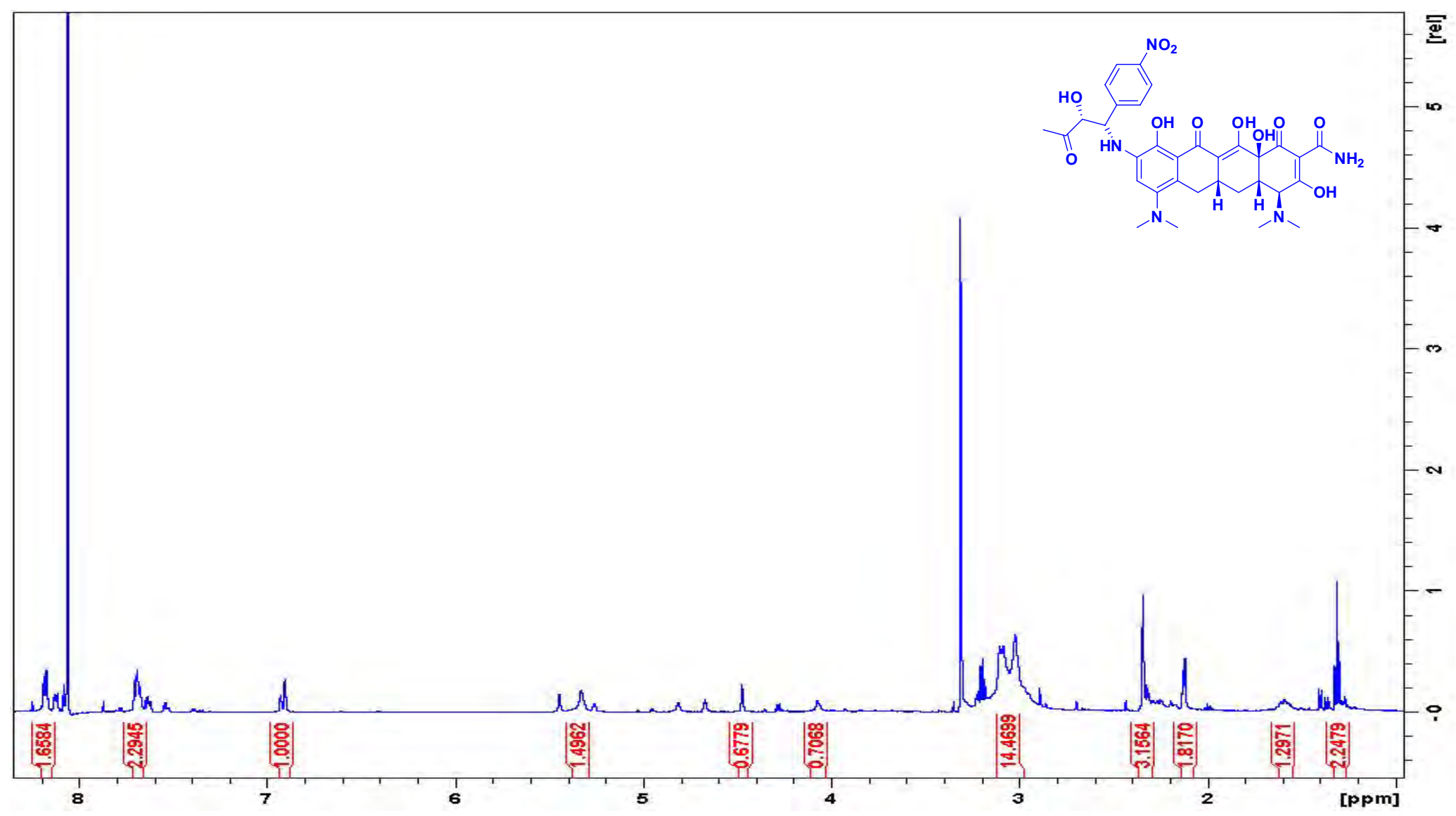


${ }^{1}$ H NMR of (4S,4aS,5aR,12aS)-9-((1S,2R)-1-(4-butoxyphenyl)-2-hydroxy-3-oxobutylamino)-4,7 bis(dimethylamino)3,10,12,12a-tetrahydroxy-1,11-dioxo-1,4,4a,5,5a,6,11,12a-octahydrotetracene-2-carboxamide (2b)

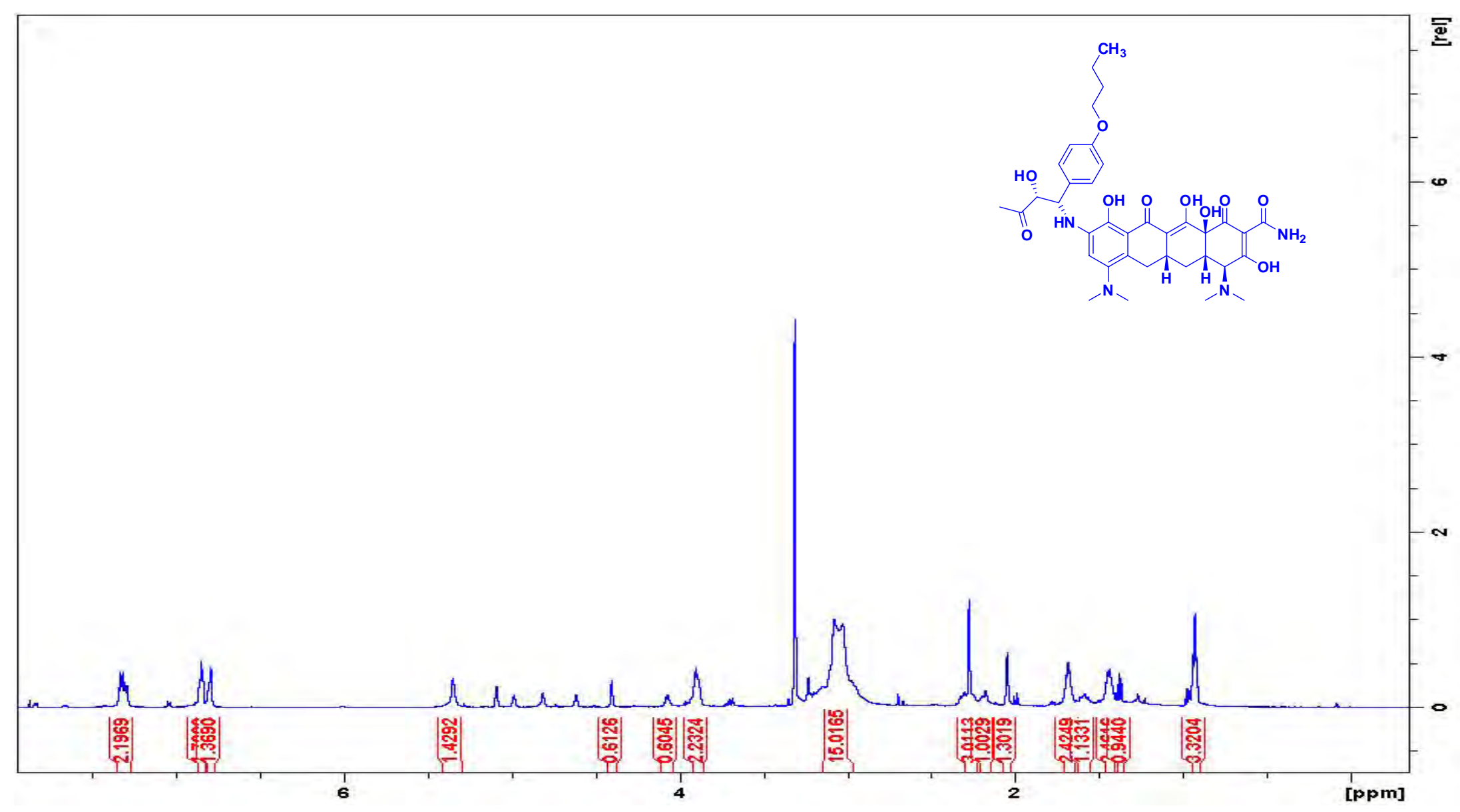


${ }^{1} \mathrm{H}$ NMR of (4S,4aS,5aR,12aS)-4,7-bis(dimethylamino)-3,10,12,12a-tetrahydroxy-9-((1S,2R)-2-hydroxy-3-oxo-1-otolylbutylamino)-1,11-dioxo-1,4,4a,5,5a,6,11,12a-octahydrotetracene-2-carboxamide (2c)

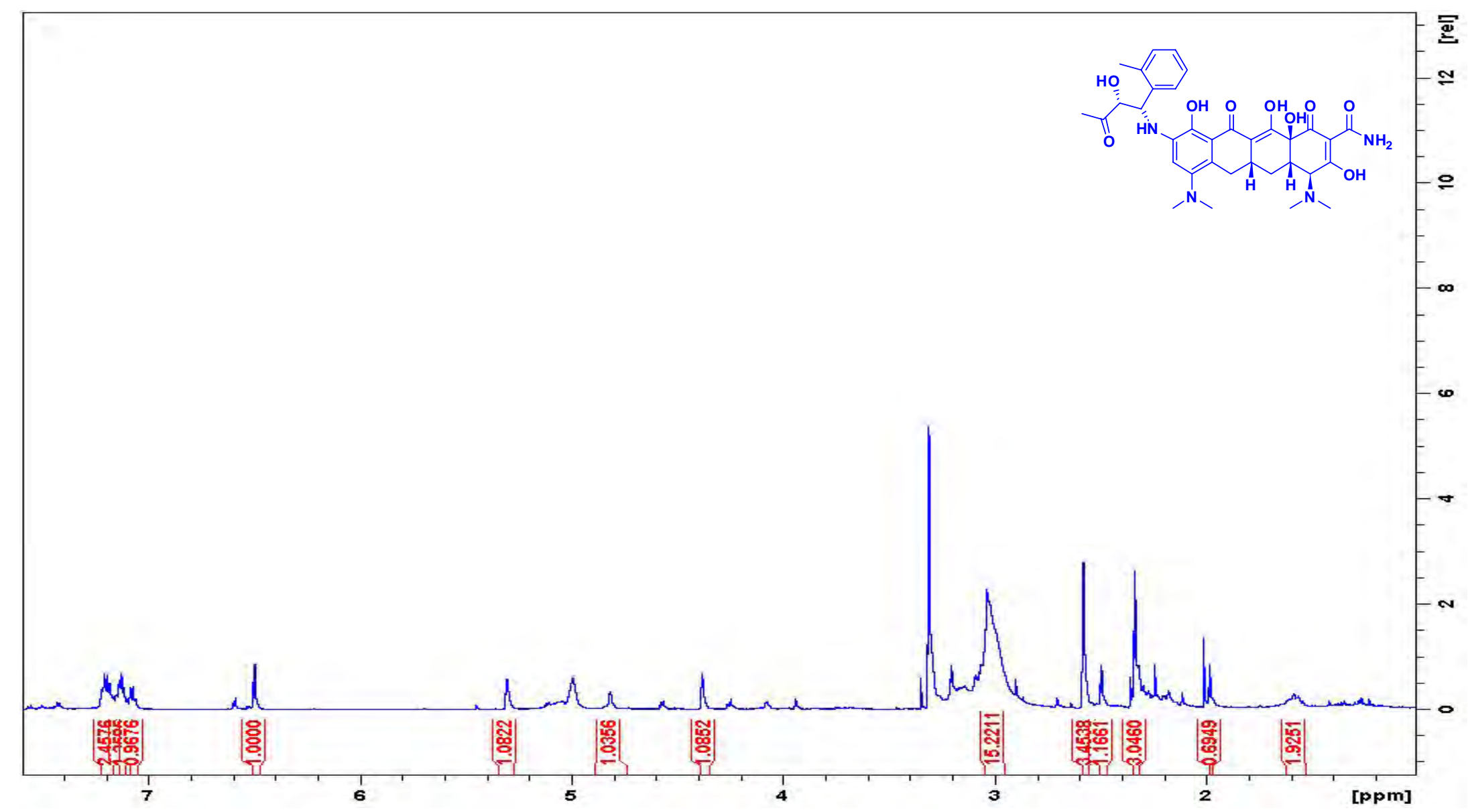


${ }^{1} \mathrm{H}$ NMR of (4S,4aS,5aR,12aS)-4,7-bis(dimethylamino)-9-((1S,2R)-1-(4-fluorophenyl)-2-hydroxy-3-oxobutylamino)3,10,12,12a-tetrahydroxy-1,11-dioxo-1,4,4a,5,5a,6,11,12a-octahydrotetracene-2-carboxamide (2d)

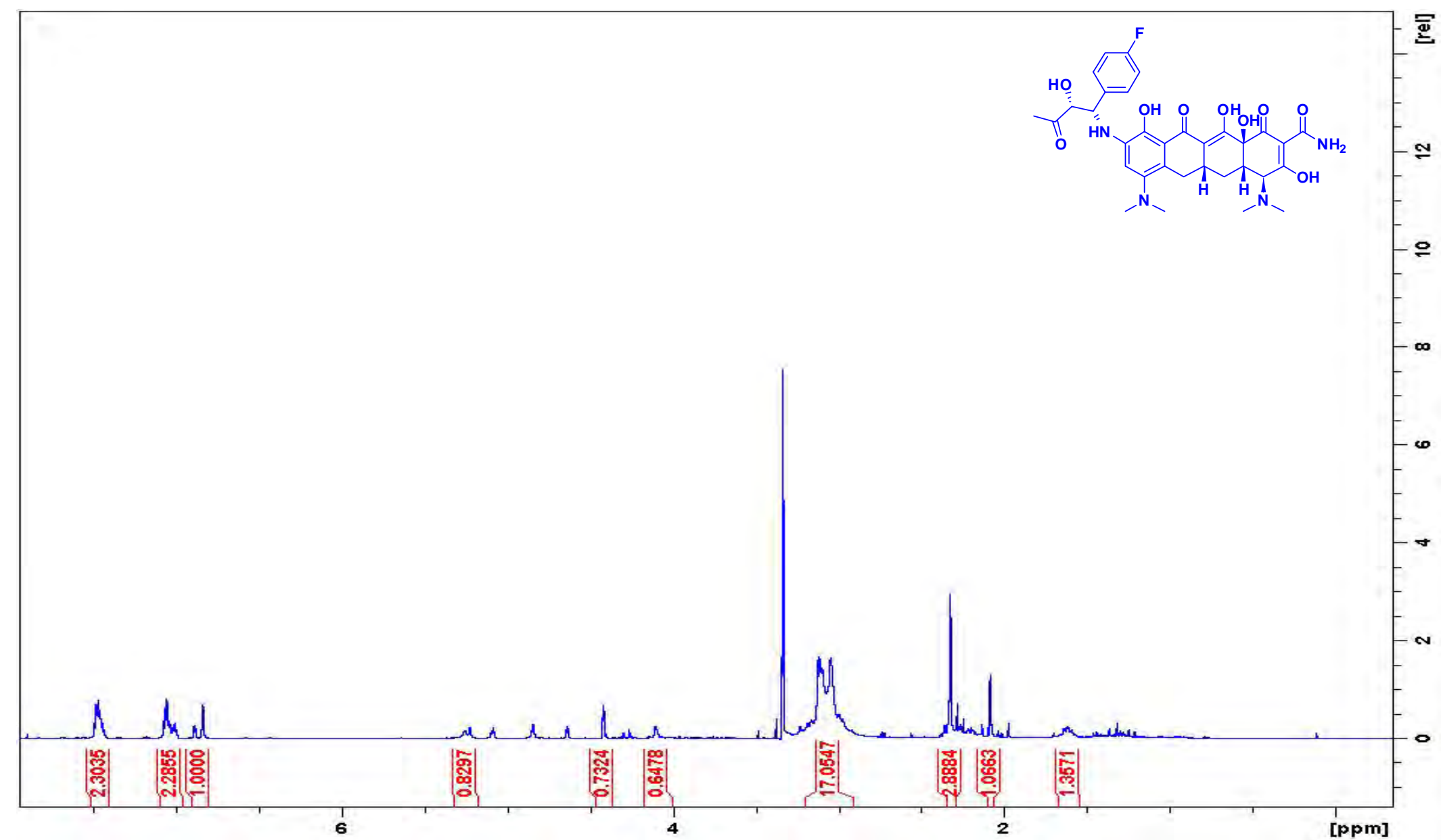


${ }^{1}$ H NMR of (4S,4aS,5aR,12aS)-4,7-bis(dimethylamino)-3,10,12,12a-tetrahydroxy-9-((1S,2R)-2-hydroxy-1-(naphthalen-2-yl)-3oxobutylamino)-1,11-dioxo-1,4,4a,5,5a,6,11,12a-octahydrotetracene-2-carboxamide (2e)

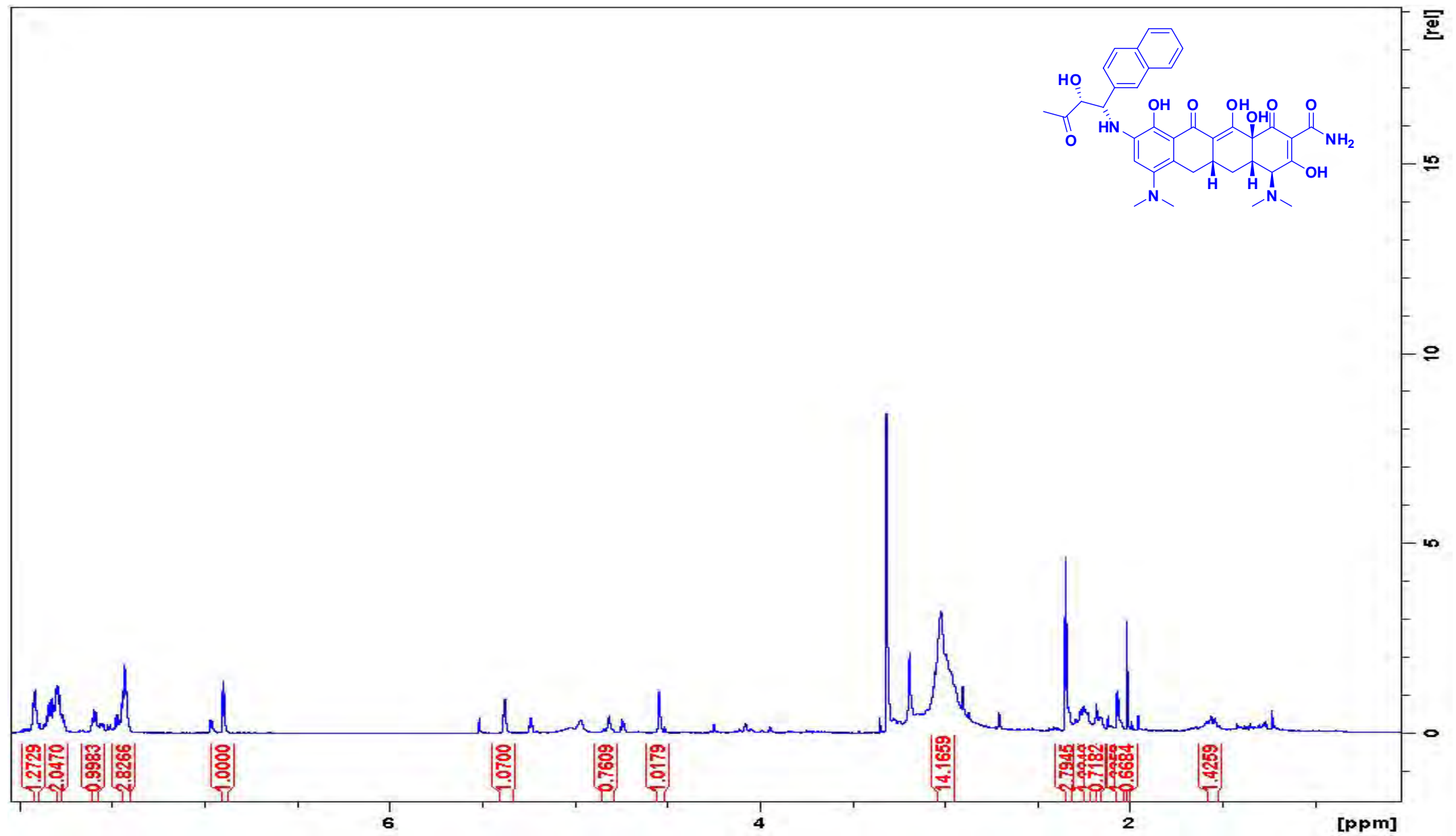


${ }^{1} \mathrm{H}$ NMR of (4S,4aS,5aR,12aS)-4,7-bis(dimethylamino)-3,10,12,12a-tetrahydroxy-9-((1R,2R)-2-hydroxy-1-(5-nitrofuran-2-yl)3-oxobutylamino)-1,11-dioxo-1,4,4a,5,5a,6,11,12a-octahydrotetracene-2-carboxamide (2f)

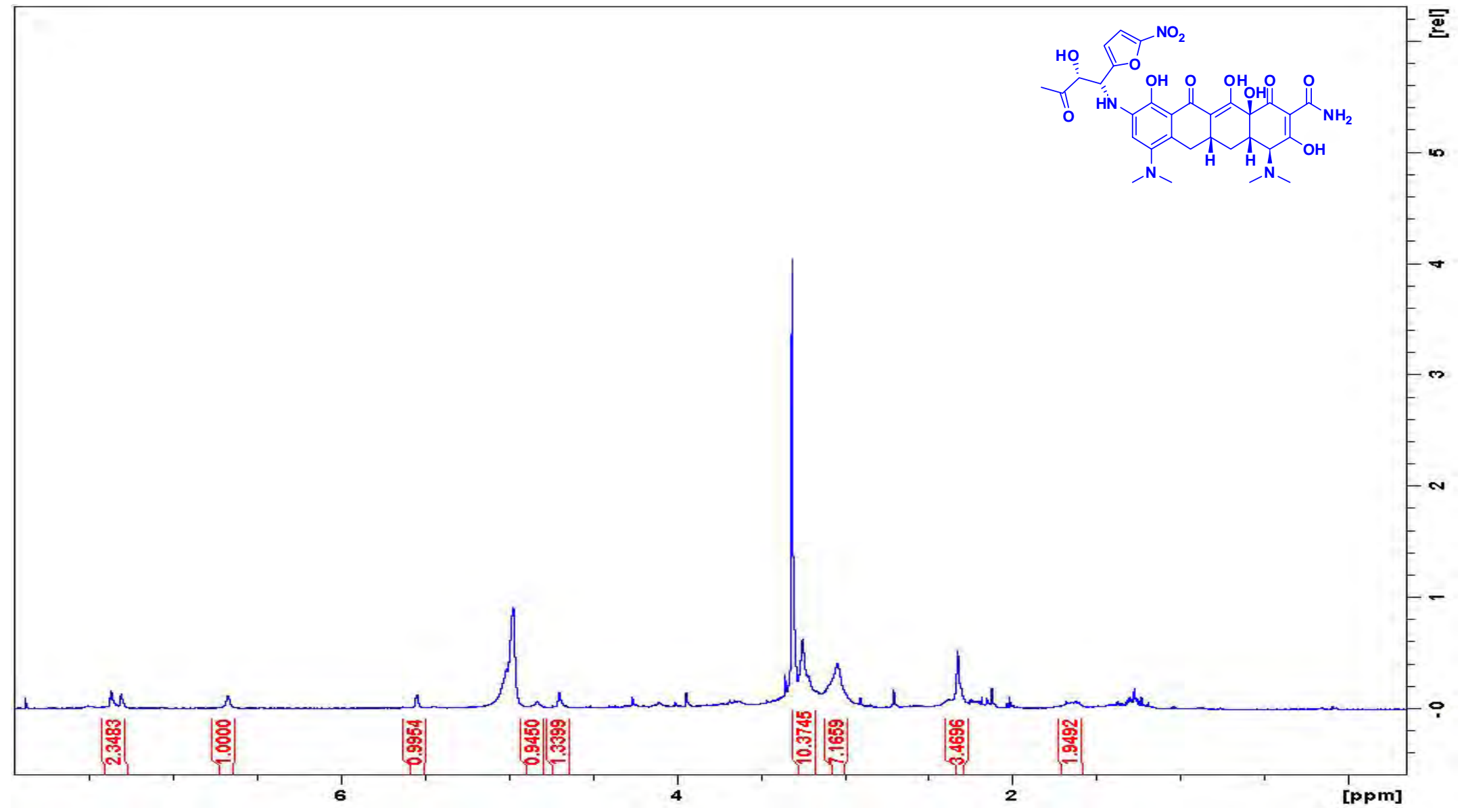


${ }^{1} \mathrm{H}$ NMR of (4S,4aS,5aR,12aS)-4,7-bis(dimethylamino)-9-((1R,2R)-1-(furan-2-yl)-2-hydroxy-3-oxobutylamino)-3,10,12,12atetrahydroxy-1,11-dioxo-1,4,4a,5,5a,6,11,12a-octahydrotetracene-2-carboxamide (2g)

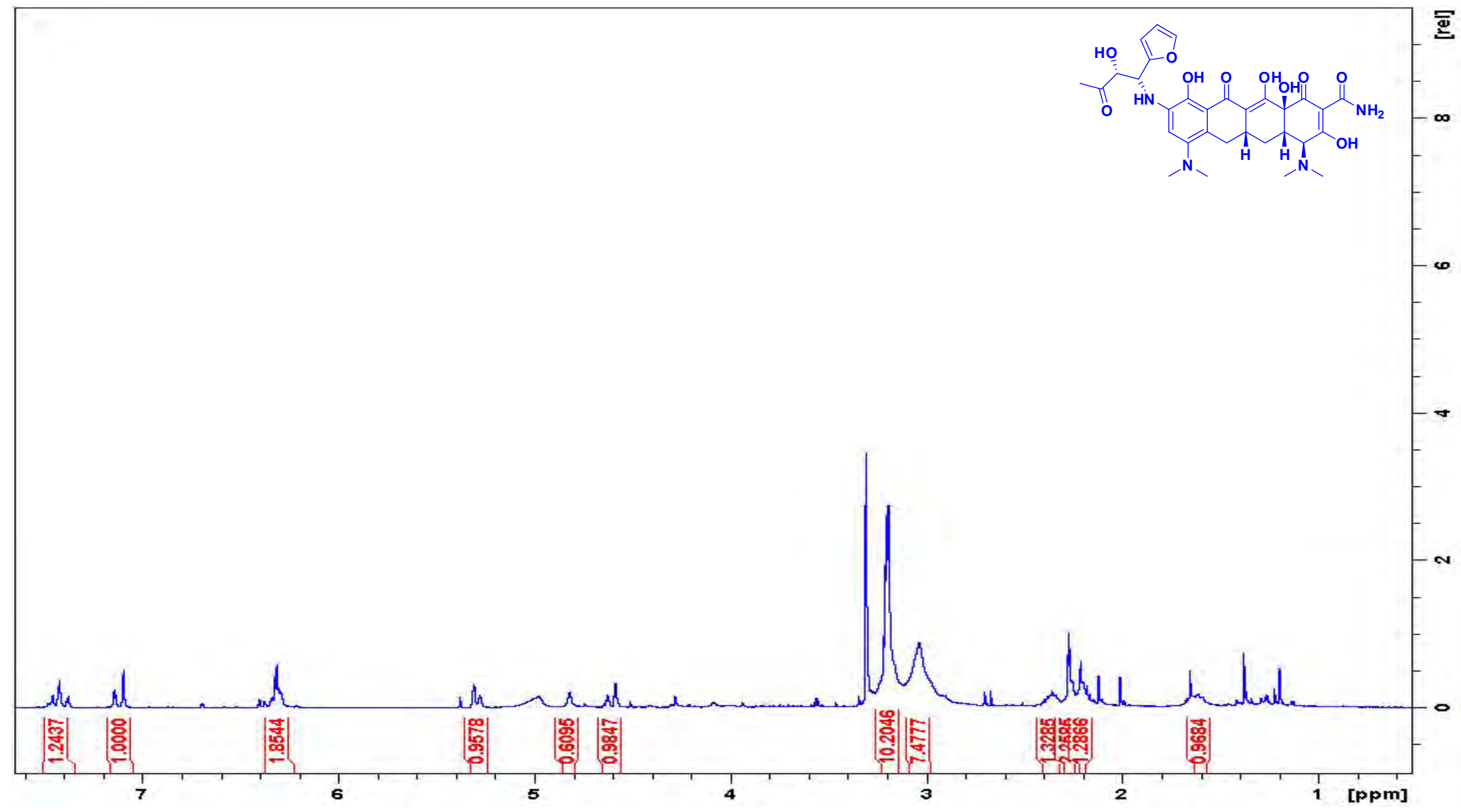


${ }^{1} \mathrm{H}$ NMR of (4S,4aS,5aR,12aS)-9-(1-(5-bromofuran-2-yl)-2-hydroxy-3-oxobutylamino)-4,7-bis (dimethylamino)-3,10,12,12atetrahydroxy-1,11-dioxo-1,4,4a,5,5a,6,11,12a-octahydrotetracene-2-carboxamide (2h)

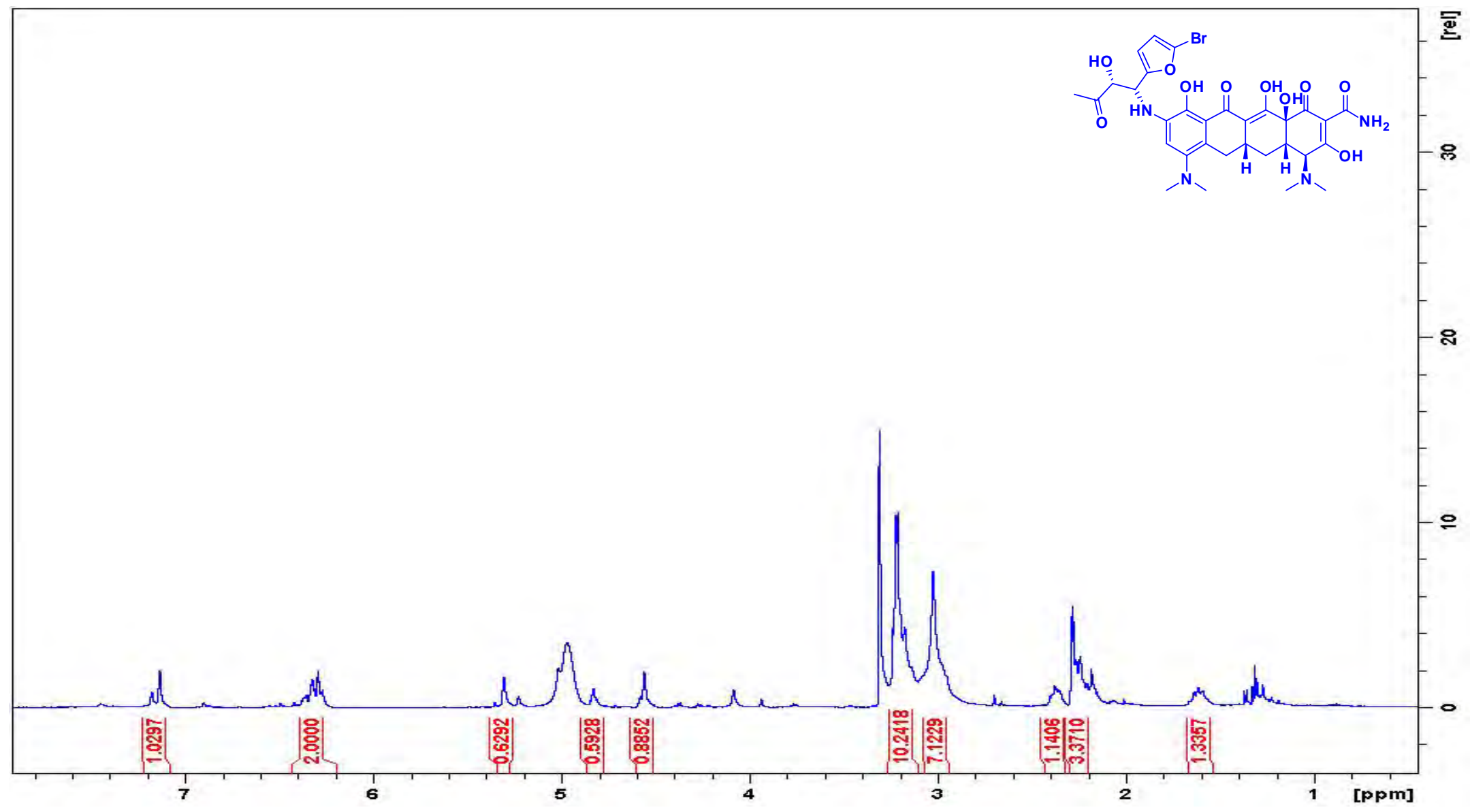


Organocatalyzed Mannich Reactions on Minocycline: Towards Novel Tetracycline Antibiotics

Tirivashe E. Chiwunze ${ }^{[a]}$, Rafiatu Azumah ${ }^{[a]}$, Melissa Ramtahal ${ }^{[a]}$, Per I. Arvidsson ${ }^{[b]}$, Sabiha Y. Essack ${ }^{[c]}$, Hendrik G. Kruger ${ }^{[a]}$, Thavendran Govender ${ }^{[a]}$, Tricia Naicker ${ }^{[a]}$

${ }^{a}$ School of Pharmacy and Pharmacology, University of KwaZulu-Natal, Westville Campus, Durban 4000, South Africa.

*e-mail: Naickert1@ukzn.ac.za>

${ }^{\mathbf{b}}$ Science for Life Laboratory, Drug Discovery and Development Platform, and Division of Translational Medicine and Chemical Biology, Department of Medical Biochemistry and Biophysics, Karolinska Institutet, Stockholm, Sweden

${ }^{\mathrm{c}}$ School of Chemistry and Physics, University of KwaZulu-Natal, Westville Campus, Durban 4000, South Africa

\section{SUPPLEMETARY DATA}




\section{Contents}

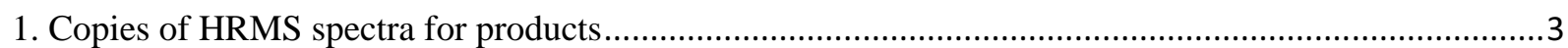

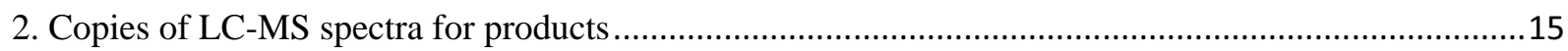

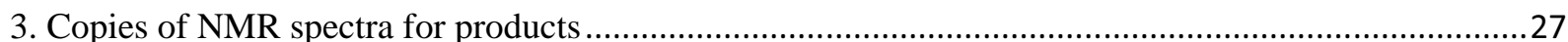




\section{Copies of HRMS spectra for products}

HRMS of (4S,4aS,5aR,12aS)-4,7-bis(dimethylamino)-3,10,12,12a-tetrahydroxy-9-(2hydroxy-3-oxo-1-phenylbutylamino)-1,11-dioxo-1,4,4a,5,5a,6,11,12a-octahydrotetracene-2carboxamide (1a)

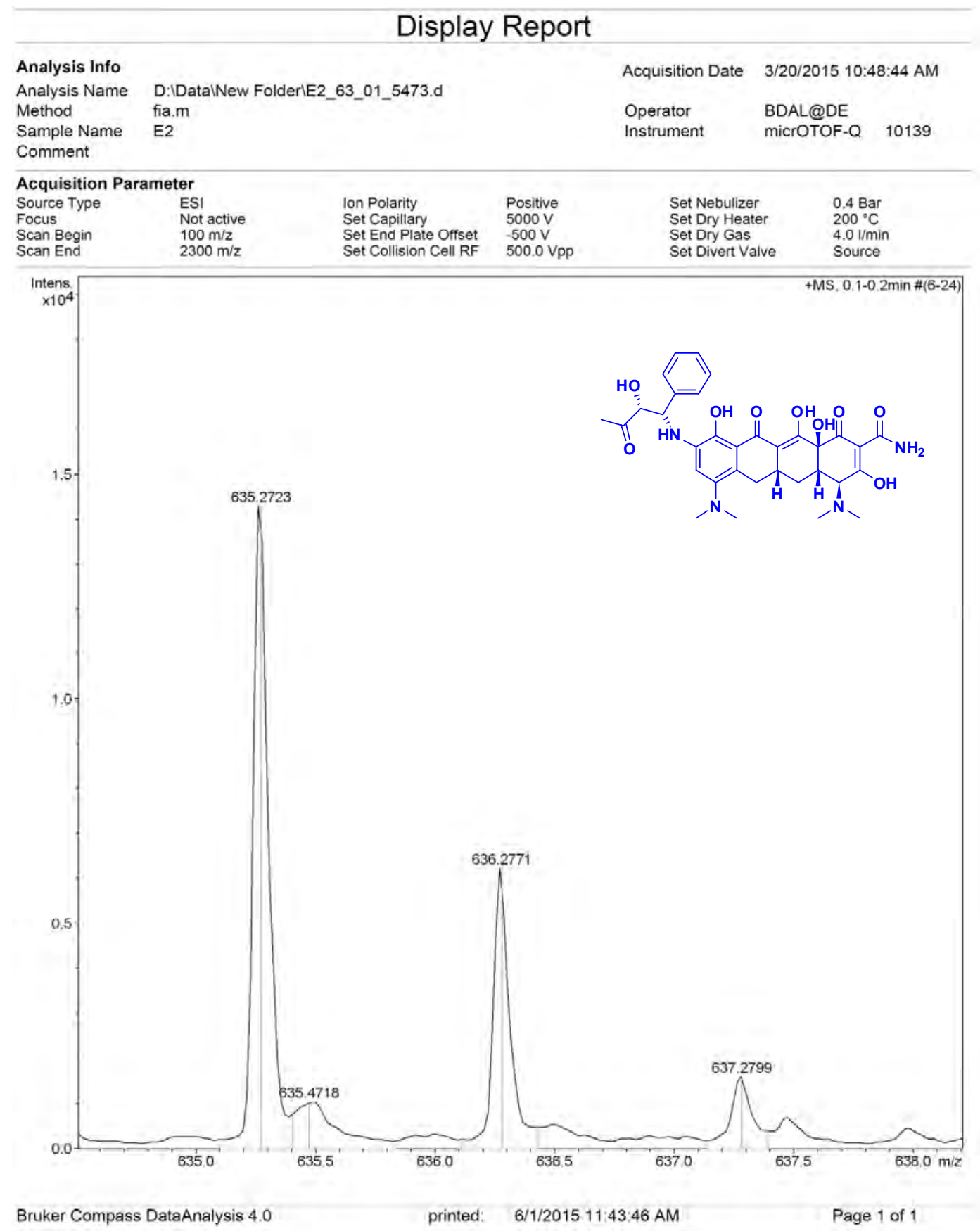


HRMS of (4S,4aS,5aR,12aS)-4,7-bis(dimethylamino)-3,10,12,12a-tetrahydroxy-9-(2methyl-3-oxo-1-phenylbutylamino)-1,11-dioxo-1,4,4a,5,5a,6,11,12a-octahydrotetracene-2carboxamide (1b)

\section{Display Report}

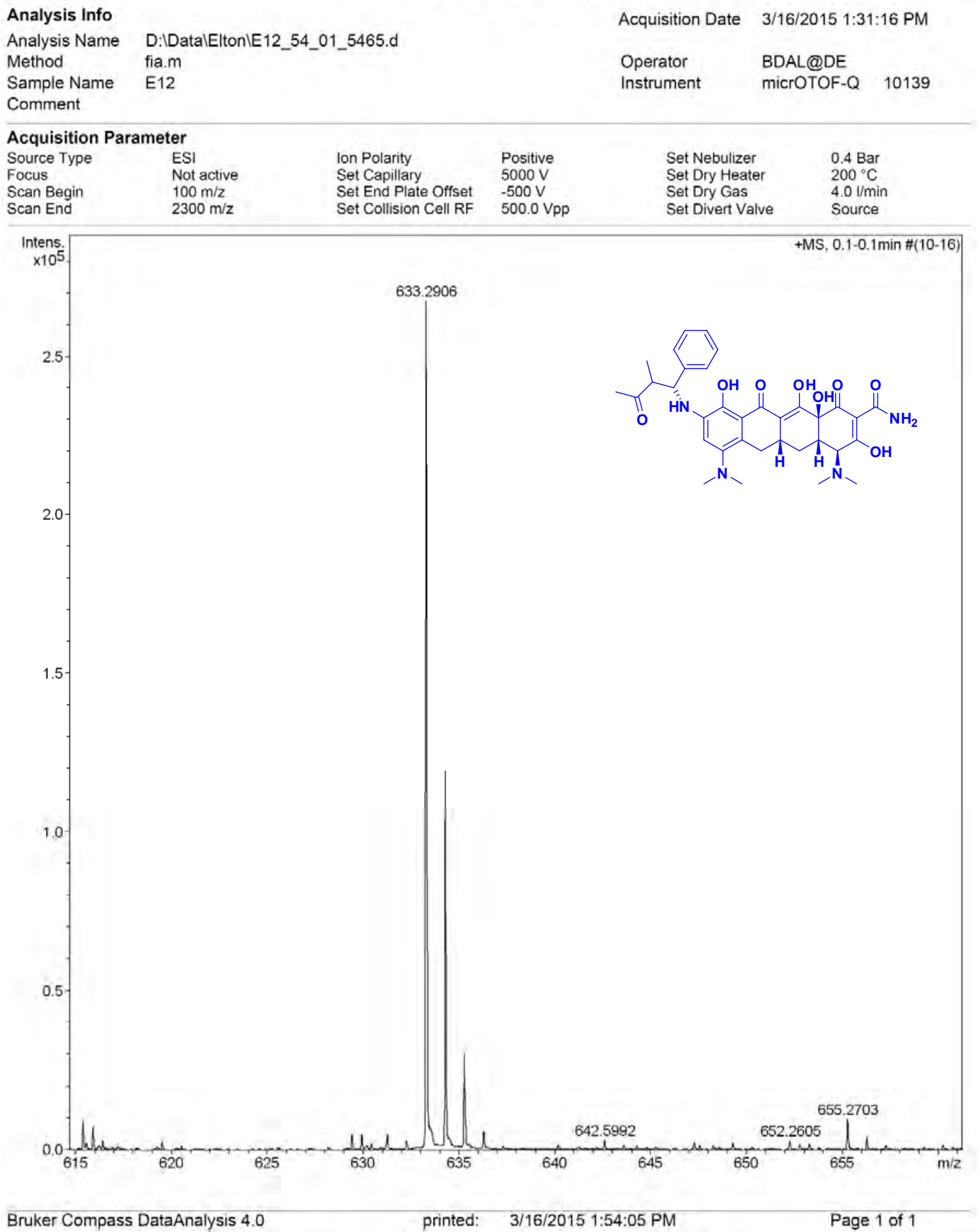


HRMS of (4S,4aS,5aR,12aS)-4,7-bis(dimethylamino)-3,10,12,12a-tetrahydroxy-1,11-dioxo-9(3-oxo-1-phenylbutylamino)-1,4,4a,5,5a,6,11,12a-octahydrotetracene-2-carboxamide (1c)

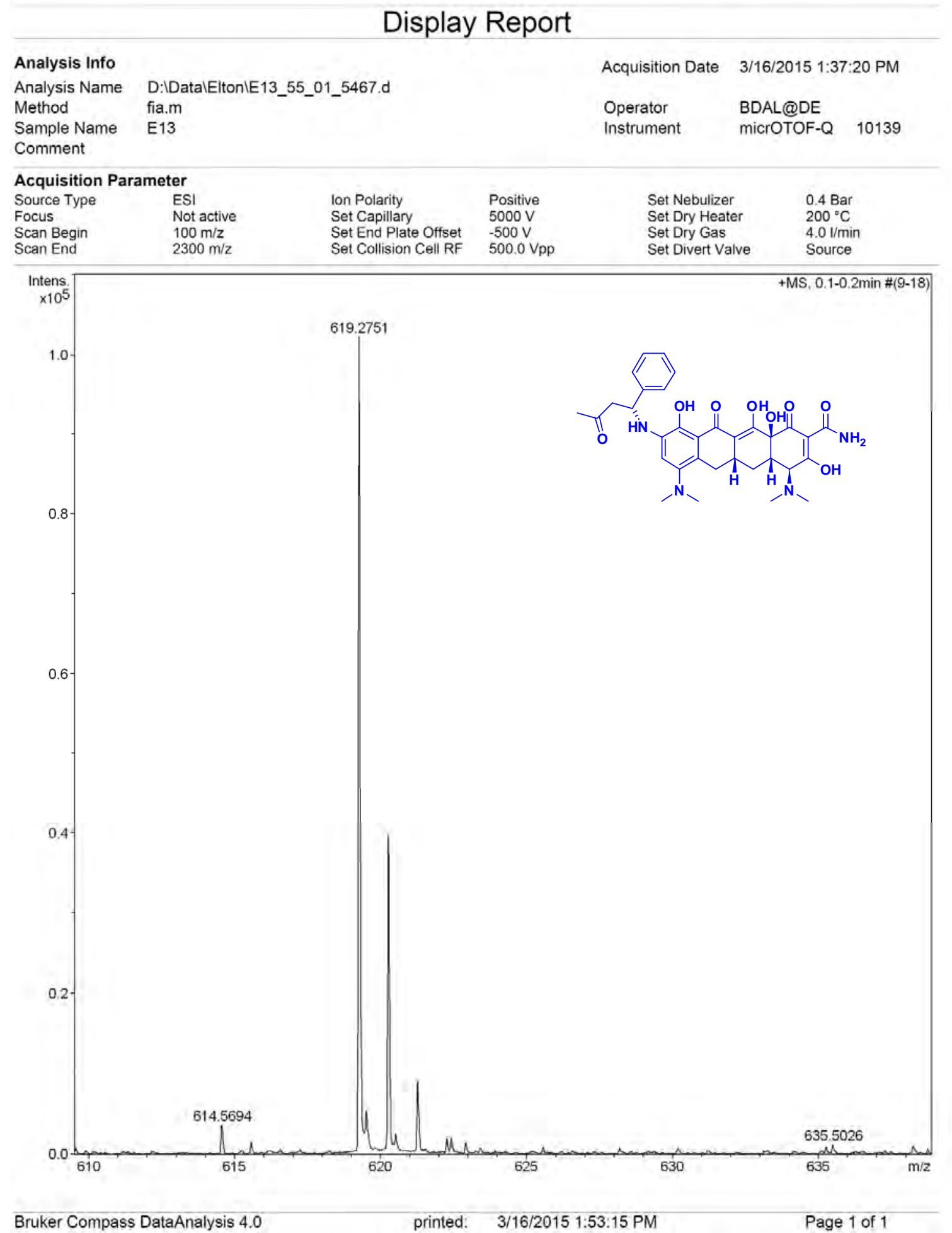


HRMS of (4S,4aS,5aR,12aS)-4,7-bis(dimethylamino)-3,10,12,12a-tetrahydroxy-1,11-dioxo-9((1R)-(2-oxocyclohexyl)(phenyl)methylamino)-1,4,4a,5,5a,6,11,12a-octahydrotetracene-2carboxamide (1d)

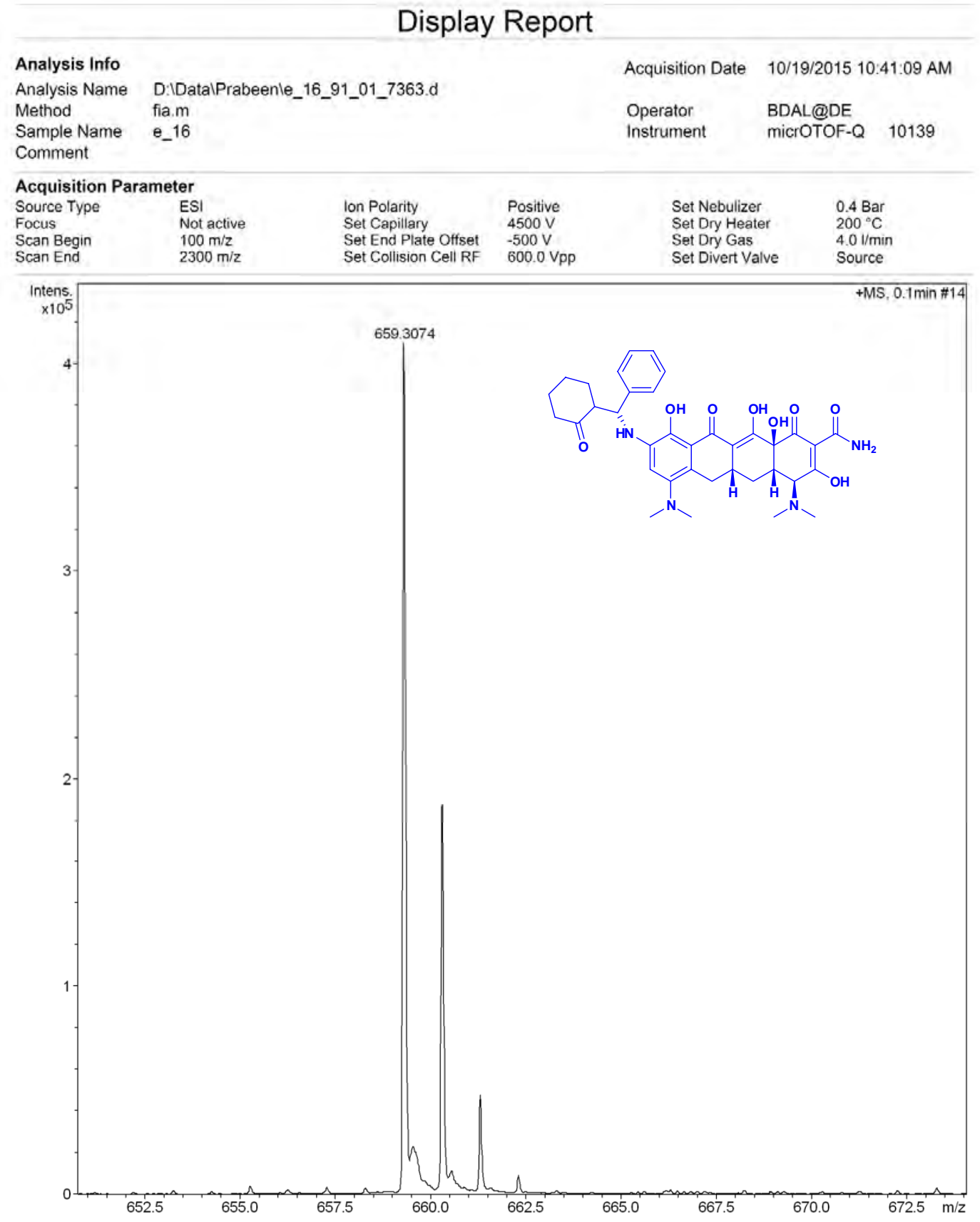


HRMS of (4S,4aS,5aR,12aS)-4,7-bis(dimethylamino)-3,10,12,12a-tetrahydroxy-9-((1S,2R)2-hydroxy-1-(4-nitrophenyl)-3-oxobutylamino)-1,11-dioxo-1,4,4a,5,5a,6,11,12aoctahydrotetracene-2-carboxamide (2a)

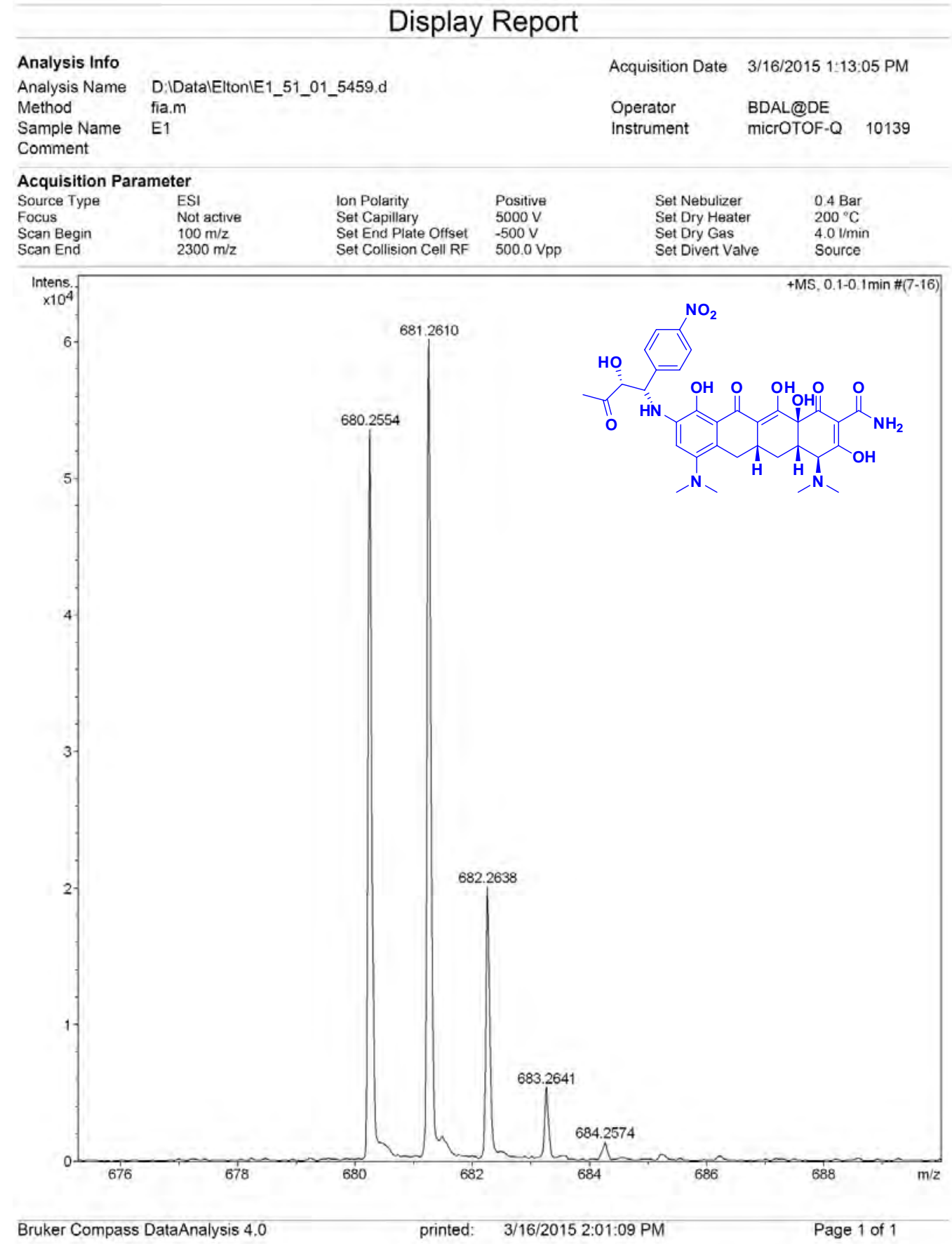


HRMS of (4S,4aS,5aR,12aS)-9-((1S,2R)-1-(4-butoxyphenyl)-2-hydroxy-3-oxobutylamino)4,7 bis(dimethylamino)-3,10,12,12a-tetrahydroxy-1,11-dioxo-1,4,4a,5,5a,6,11,12aoctahydrotetracene-2-carboxamide (2b)

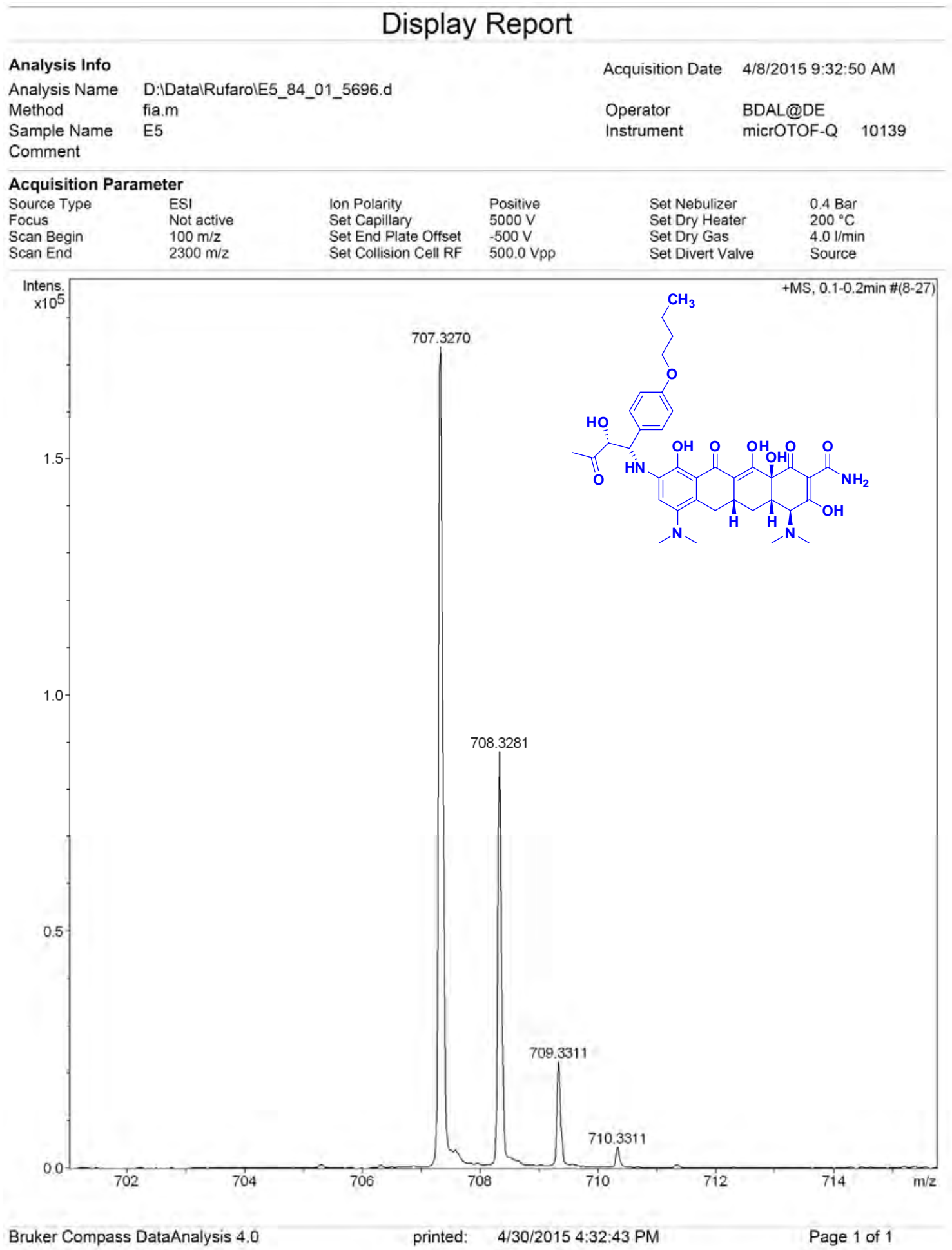


HRMS of (4S,4aS,5aR,12aS)-4,7-bis(dimethylamino)-3,10,12,12a-tetrahydroxy-9-((1S,2R)2-hydroxy-3-oxo-1-o-tolylbutylamino)-1,11-dioxo-1,4,4a,5,5a,6,11,12a-octahydrotetracene2-carboxamide (2c)

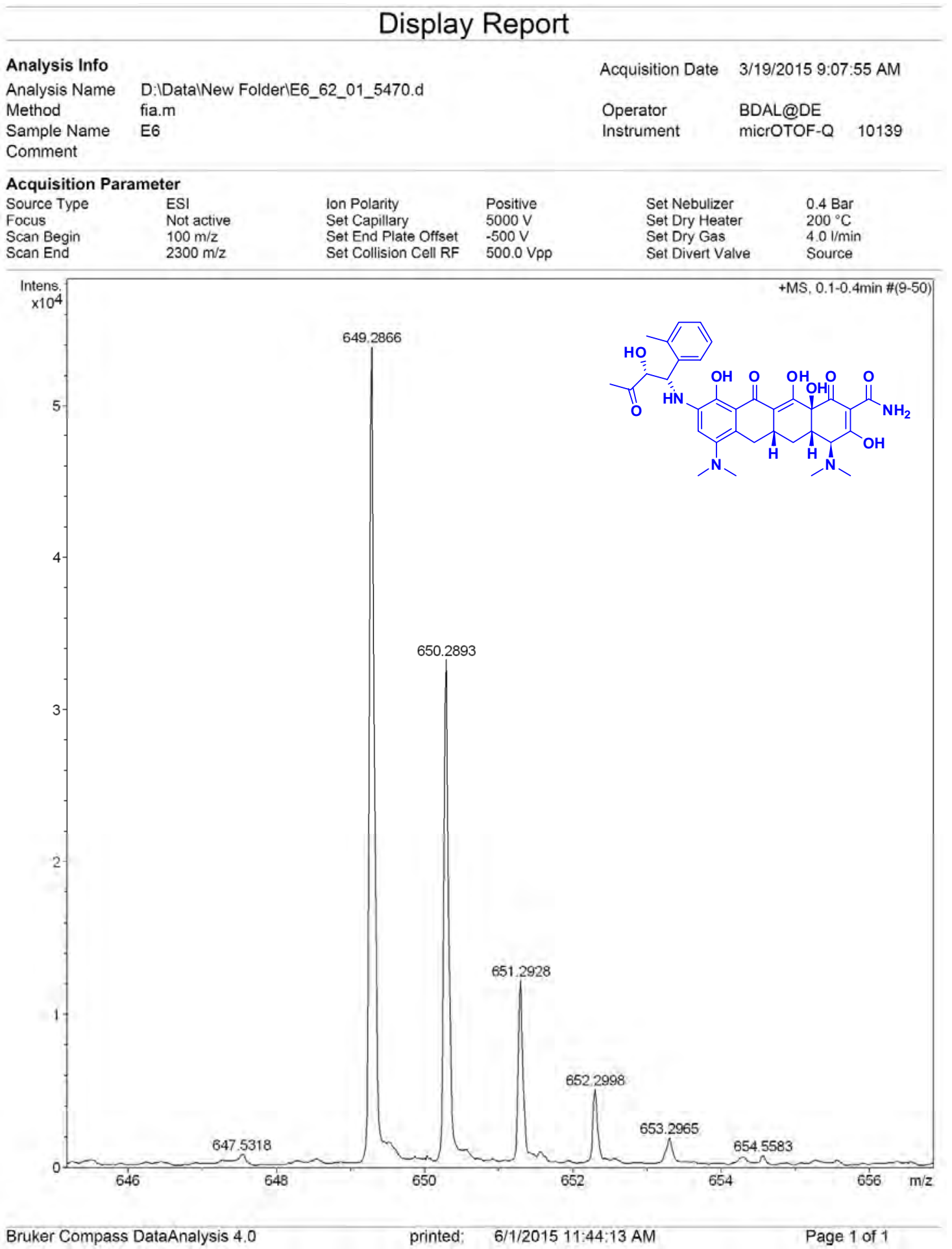


HRMS of (4S,4aS,5aR,12aS)-4,7-bis(dimethylamino)-9-((1S,2R)-1-(4-fluorophenyl)-2hydroxy-3-oxobutylamino)-3,10,12,12a-tetrahydroxy-1,11-dioxo-1,4,4a,5,5a,6,11,12aoctahydrotetracene-2-carboxamide (2d)

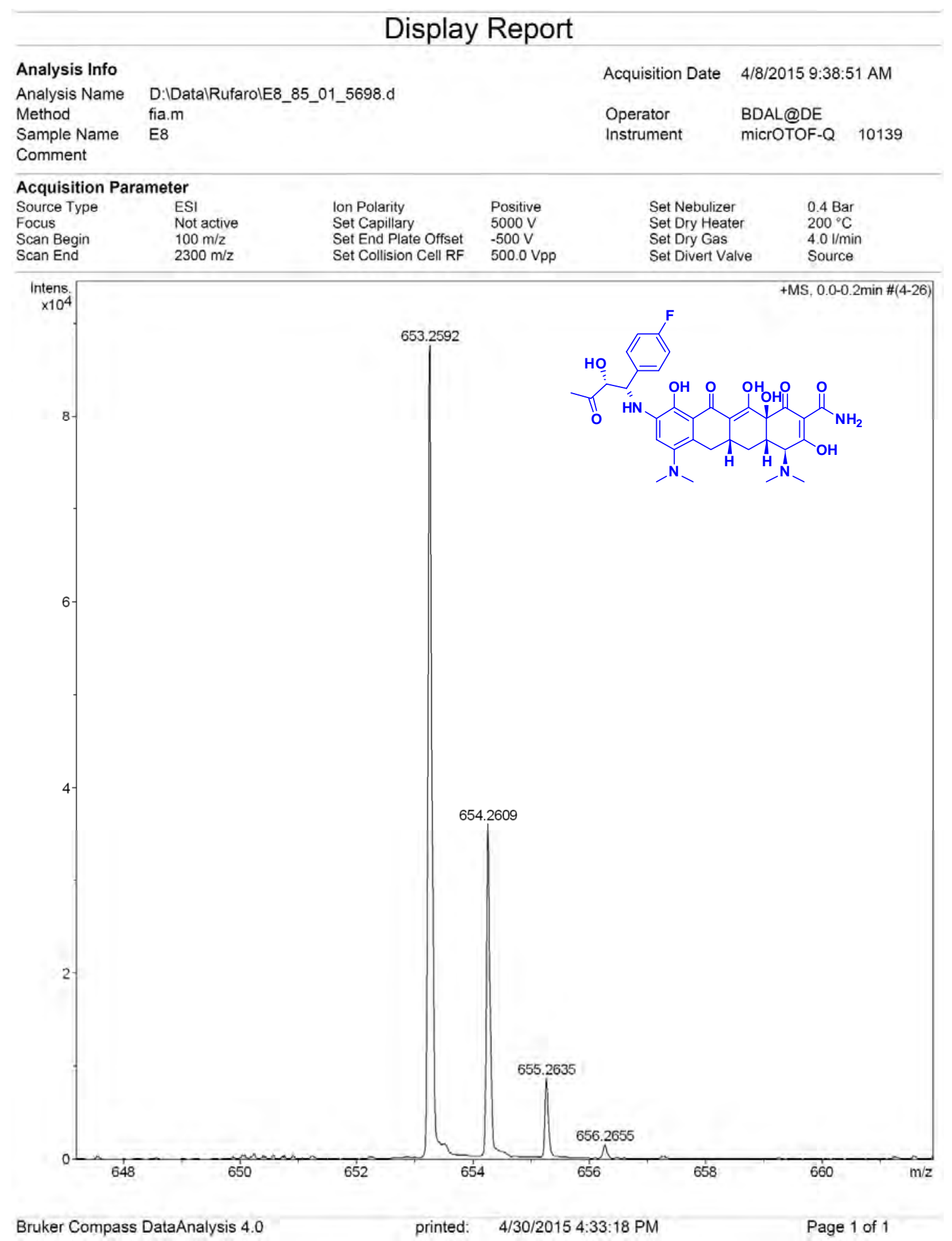


HRMS of (4S,4aS,5aR,12aS)-4,7-bis(dimethylamino)-3,10,12,12a-tetrahydroxy-9-((1S,2R)-2hydroxy-1-(naphthalen-2-yl)-3-oxobutylamino)-1,11-dioxo-1,4,4a,5,5a,6,11,12aoctahydrotetracene-2-carboxamide (2e)

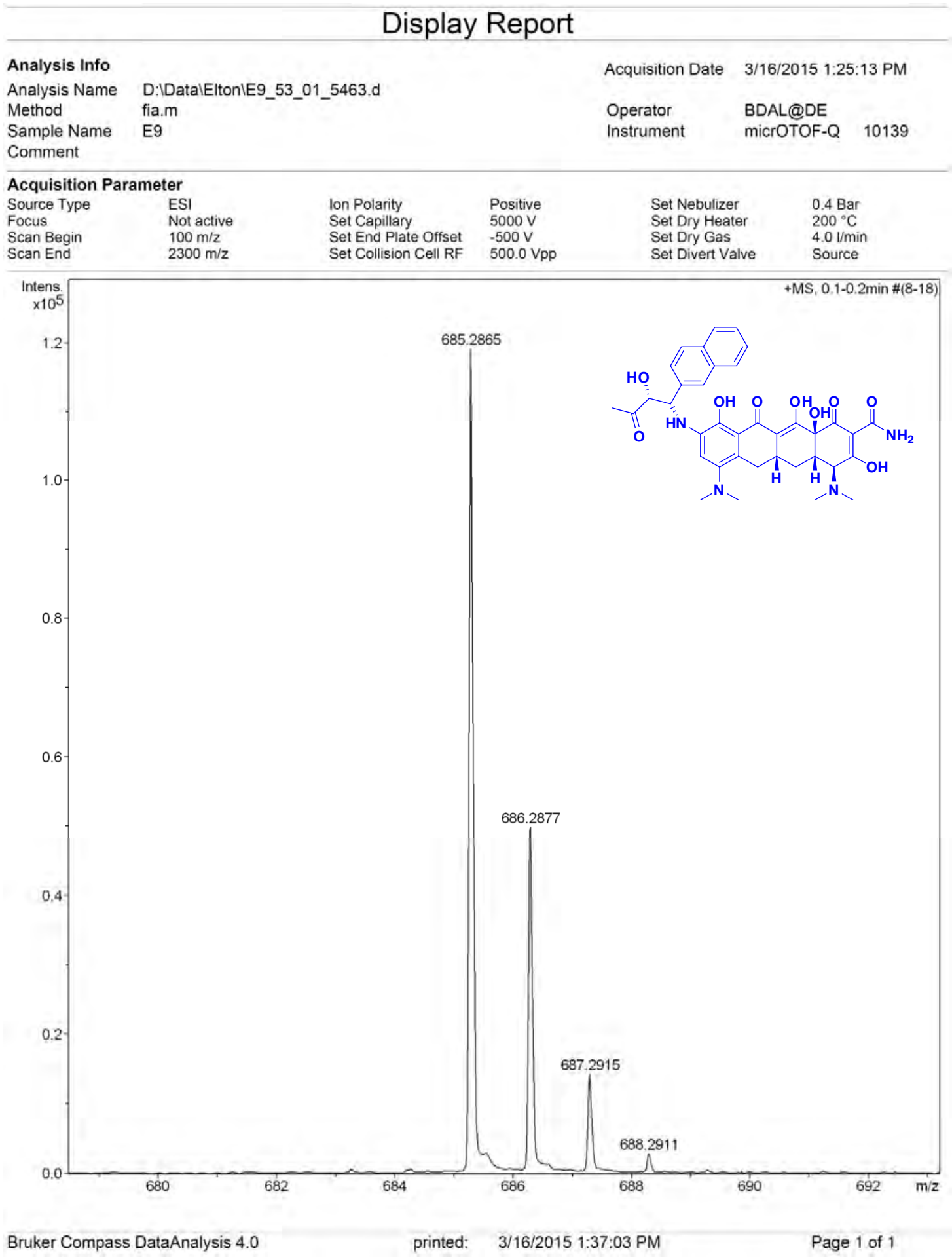


HRMS of (4S,4aS,5aR,12aS)-4,7-bis(dimethylamino)-3,10,12,12a-tetrahydroxy-9-((1R,2R)-

2-hydroxy-1-(5-nitrofuran-2-yl)-3-oxobutylamino)-1,11-dioxo-1,4,4a,5,5a,6,11,12

octahydrotetracene-2-carboxamide (2f)

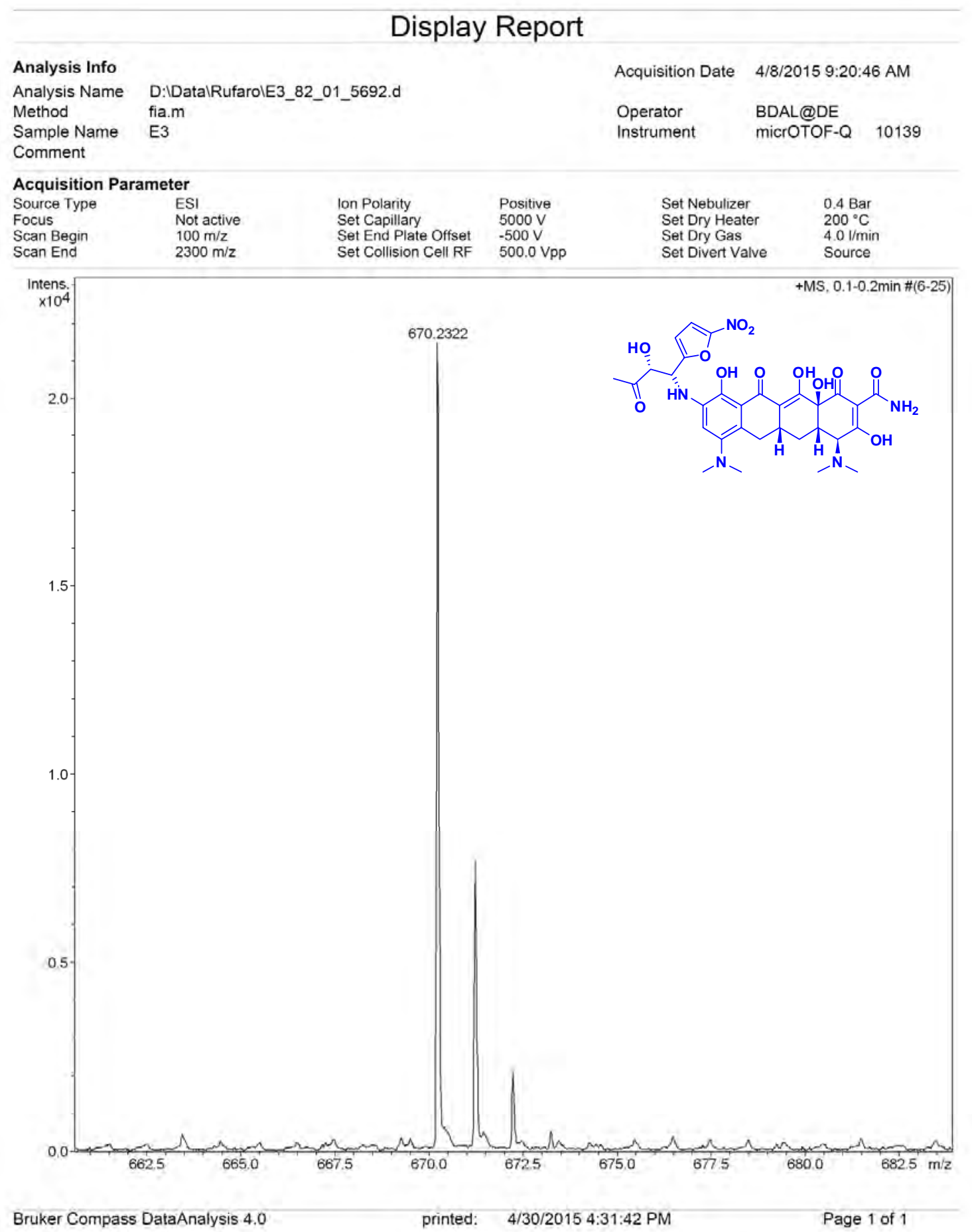


HRMS of (4S,4aS,5aR,12aS)-4,7-bis(dimethylamino)-9-((1R,2R)-1-(furan-2-yl)-2-hydroxy-

3-oxobutylamino)-3,10,12,12a-tetrahydroxy-1,11-dioxo-1,4,4a,5,5a,6,11,12aoctahydrotetracene-2-carboxamide (2g)

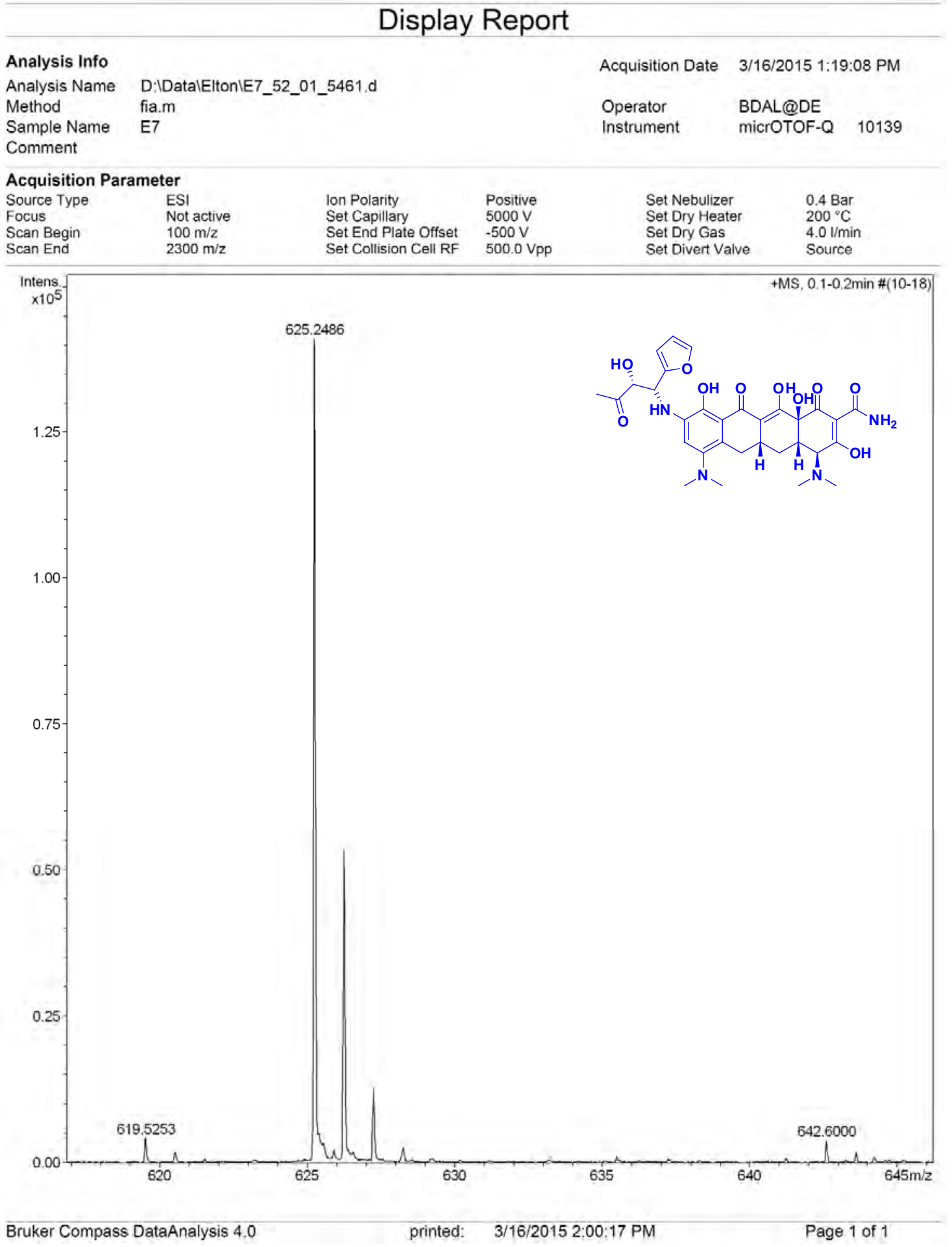


HRMS of (4S,4aS,5aR,12aS)-9-(1-(5-bromofuran-2-yl)-2-hydroxy-3-oxobutylamino)-4,7-bis (dimethylamino)-3,10,12,12a-tetrahydroxy-1,11-dioxo-1,4,4a,5,5a,6,11,12aoctahydrotetracene-2-carboxamide $(2 \mathrm{~h})$

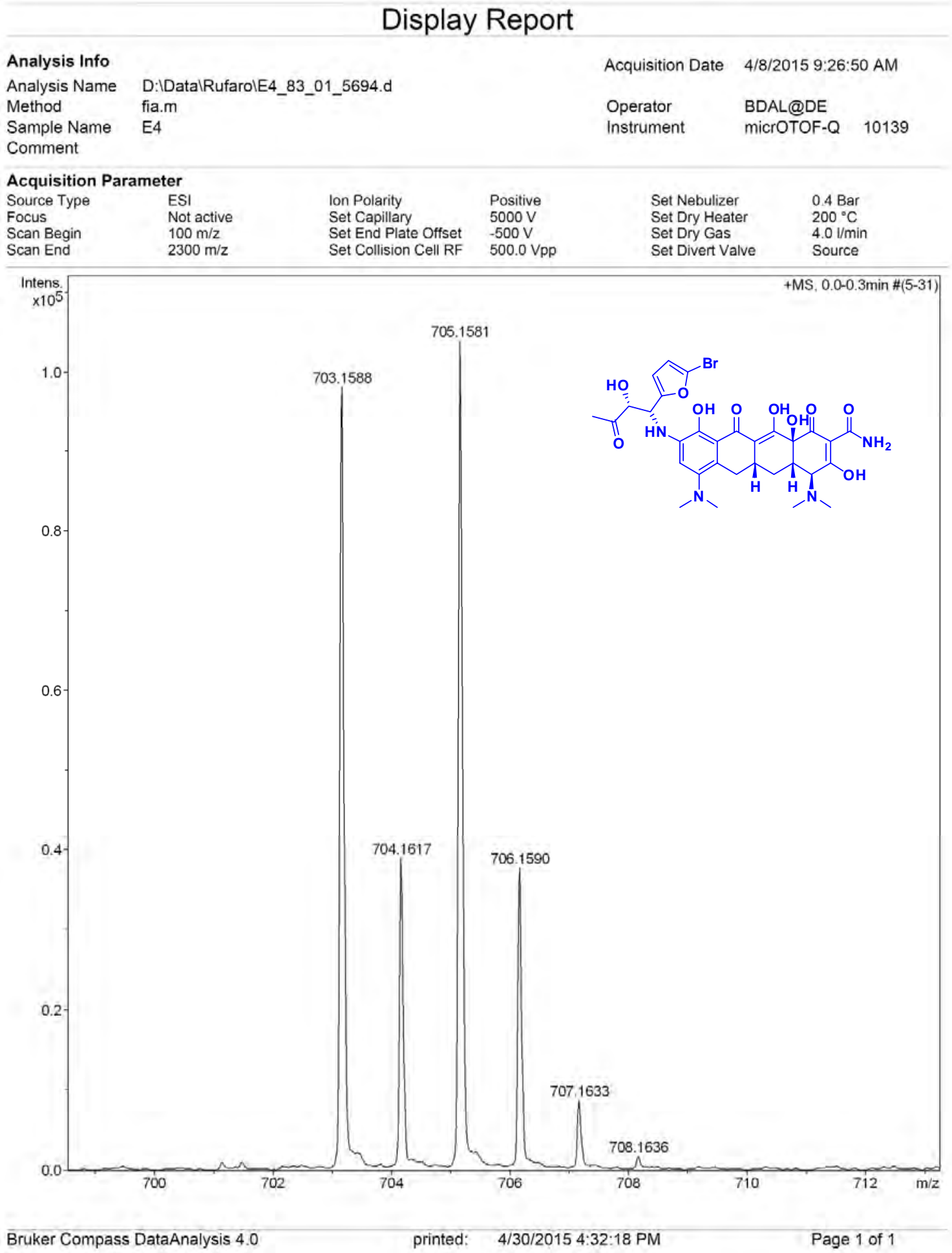




\section{Copies of LC-MS spectra for products}

LC-MS of (4S,4aS,5aR,12aS)-4,7-bis(dimethylamino)-3,10,12,12a-tetrahydroxy-9-(2hydroxy-3-oxo-1-phenylbutylamino)-1,11-dioxo-1,4,4a,5,5a,6,11,12a-octahydrotetracene-2carboxamide (1a)

\section{==== Shimadzu LabSolutions Multi-Chromatogram ====}

mAU
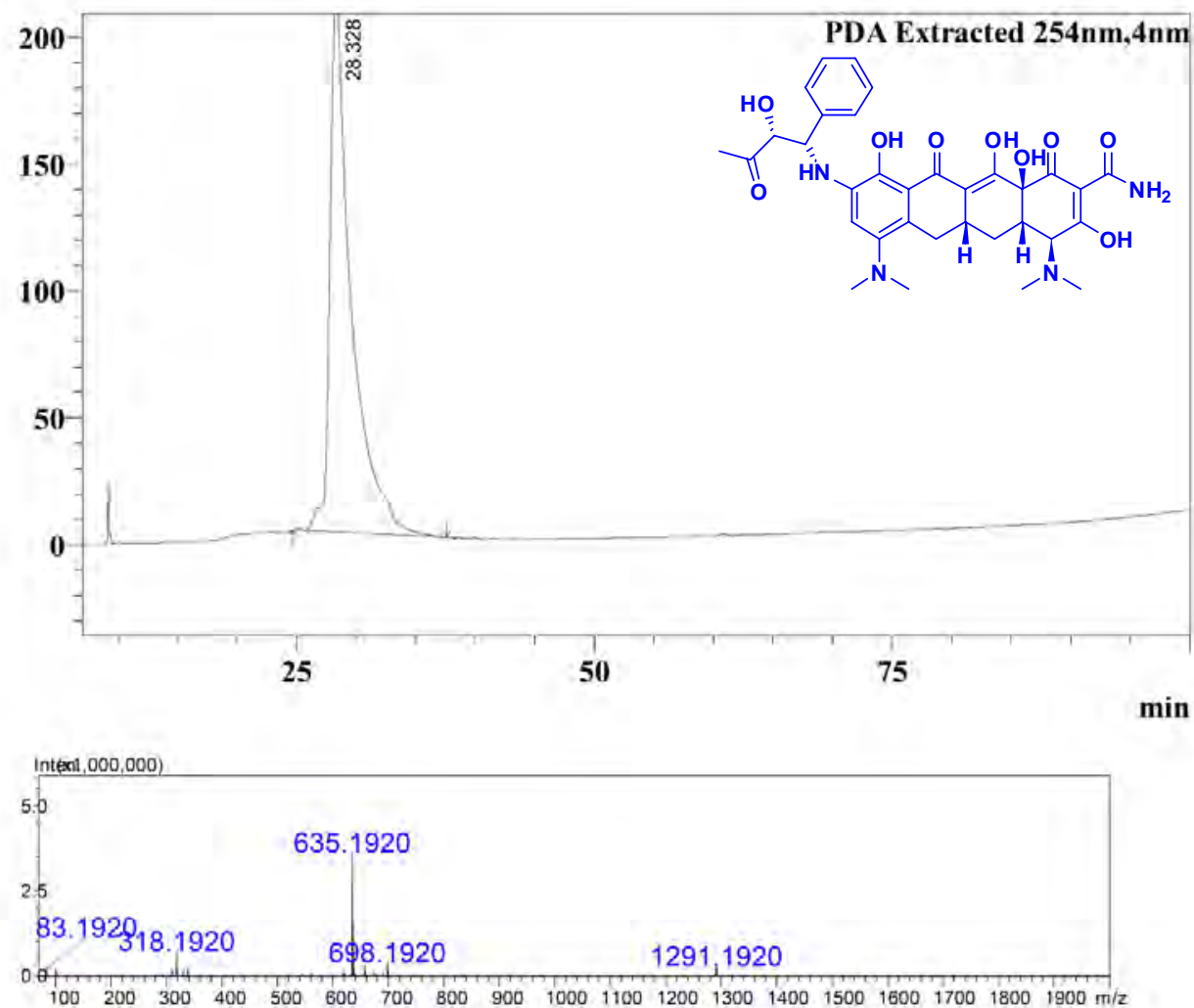
LC-MS of (4S,4aS,5aR,12aS)-4,7-bis(dimethylamino)-3,10,12,12a-tetrahydroxy-9-(2methyl-3-oxo-1-phenylbutylamino)-1,11-dioxo-1,4,4a,5,5a,6,11,12a-octahydrotetracene-2carboxamide (1b)

14/10/2015 16:06:09 Page $1 / 5$

==== Shimadzu LabSolutions Multi-Chromatogram ==== $\mathrm{mAU}$
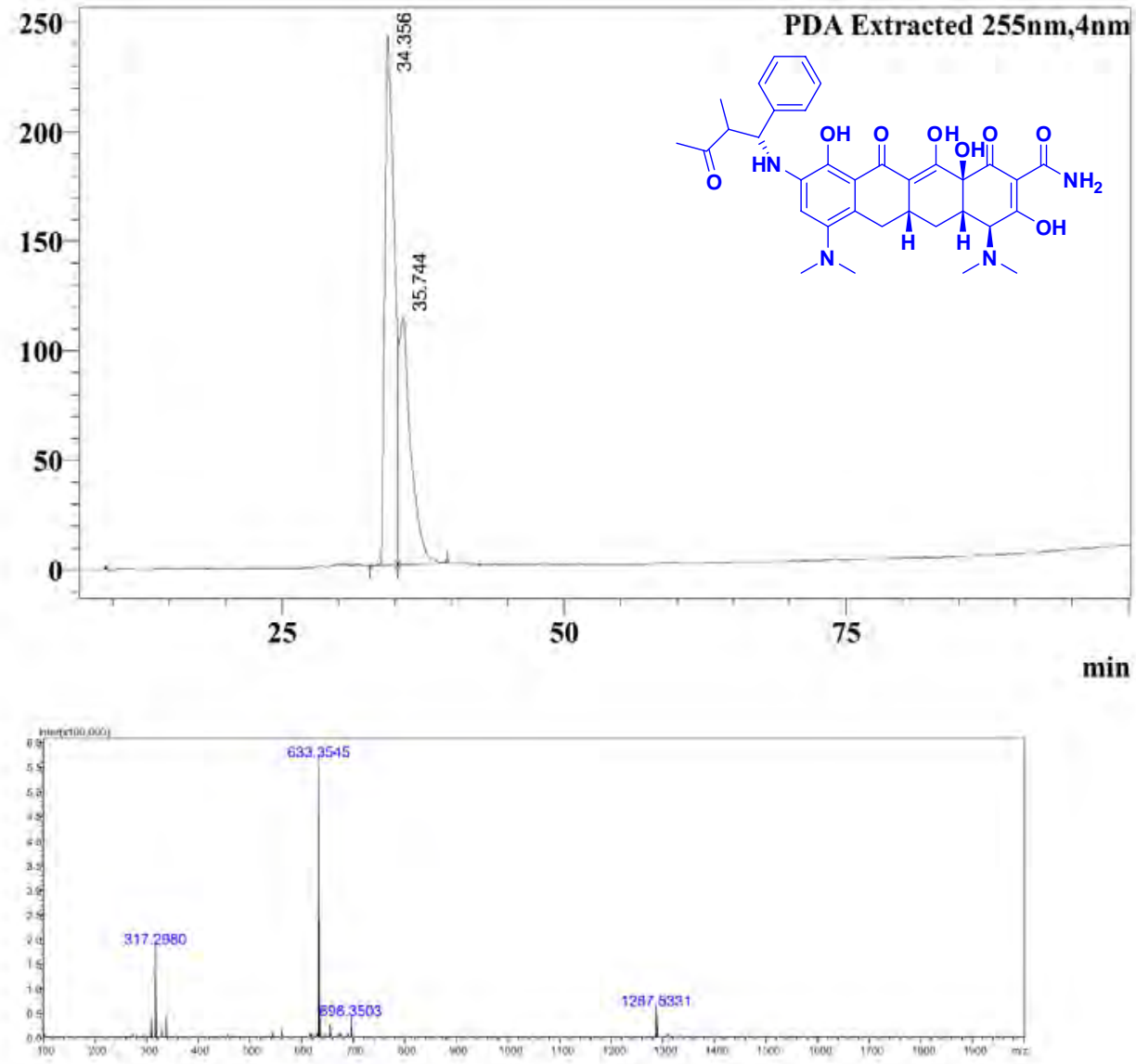
LC-MS of (4S,4aS,5aR,12aS)-4,7-bis(dimethylamino)-3,10,12,12a-tetrahydroxy-1,11-dioxo9-(3-oxo-1-phenylbutylamino)-1,4,4a,5,5a,6,11,12a-octahydrotetracene-2-carboxamide (1c)

17/10/2015 18:11:31 Page 1/5
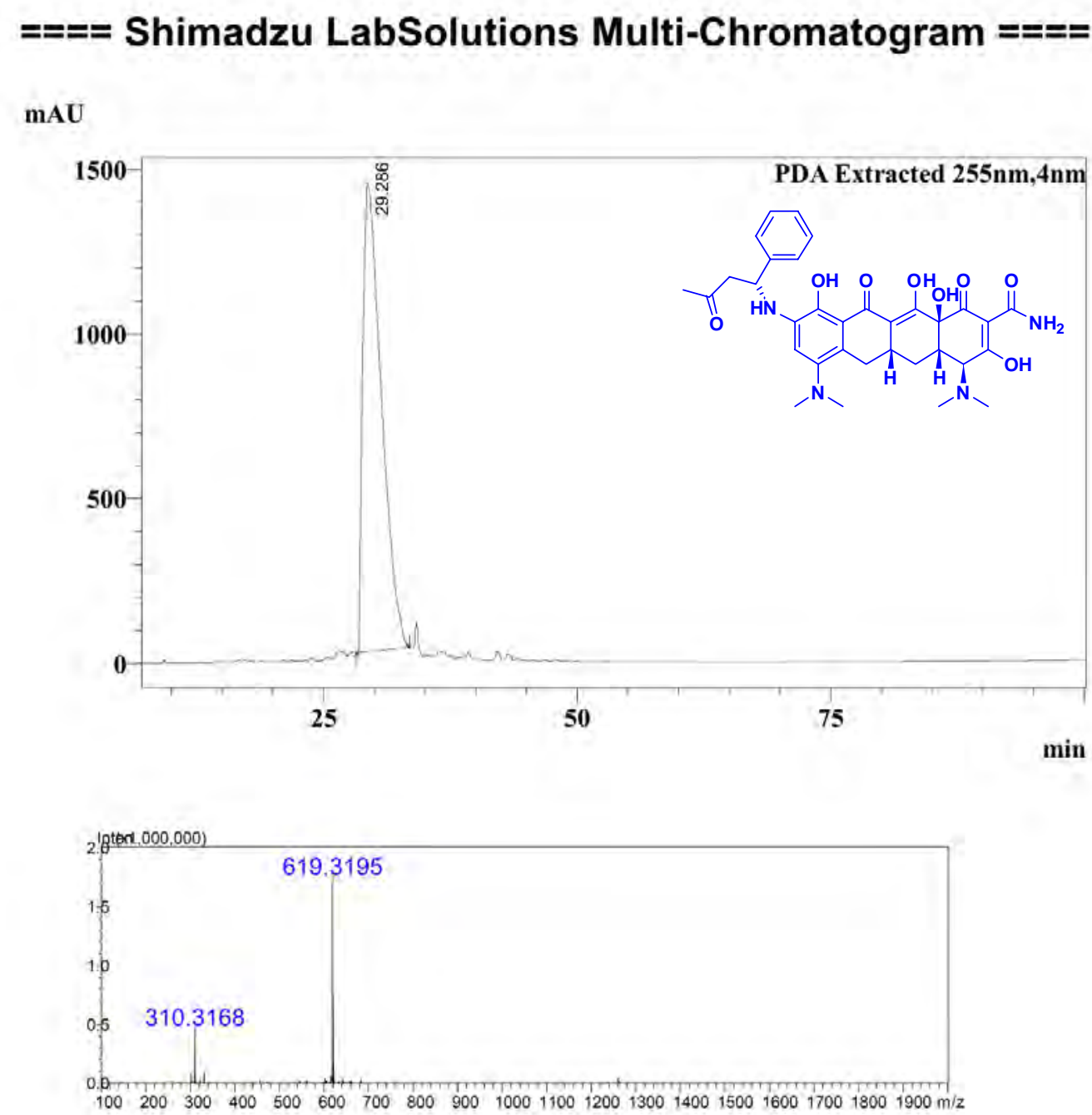
LC-MS of (4S,4aS,5aR,12aS)-4,7-bis(dimethylamino)-3,10,12,12a-tetrahydroxy-1,11-dioxo9-((1R)-(2-oxocyclohexyl)(phenyl)methylamino)-1,4,4a,5,5a,6,11,12a-octahydrotetracene-2carboxamide (1d)

\section{=ニ== Shimadzu LabSolutions Multi-Chromatogram ==== mAU}
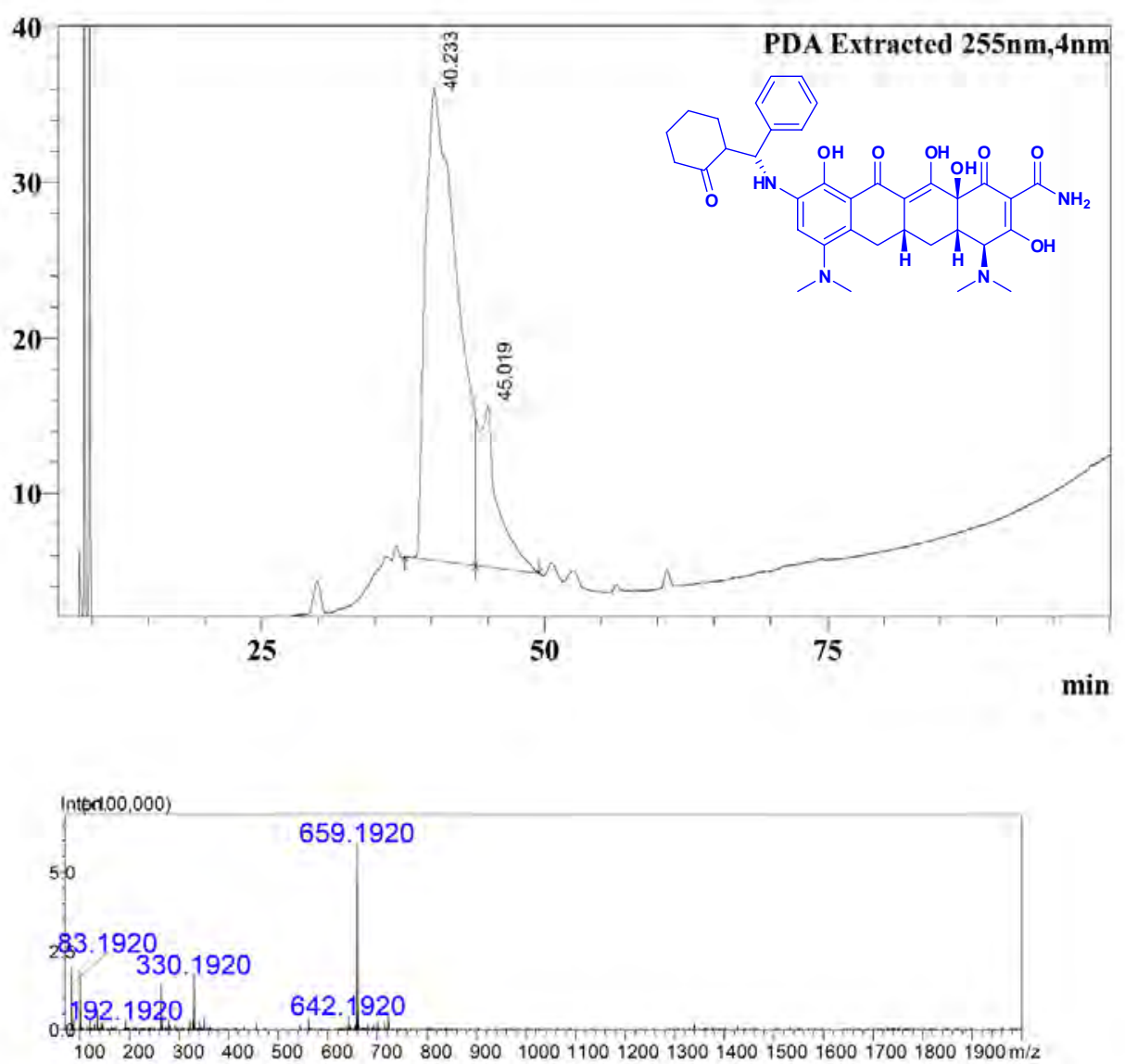
LC-MS of (4S,4aS,5aR,12aS)-4,7-bis(dimethylamino)-3,10,12,12a-tetrahydroxy-9-((1S,2R)2-hydroxy-1-(4-nitrophenyl)-3-oxobutylamino)-1,11-dioxo-1,4,4a,5,5a,6,11,12aoctahydrotetracene-2-carboxamide (2a)

\section{==== Shimadzu LabSolutions Multi-Chromatogram ====} mAU
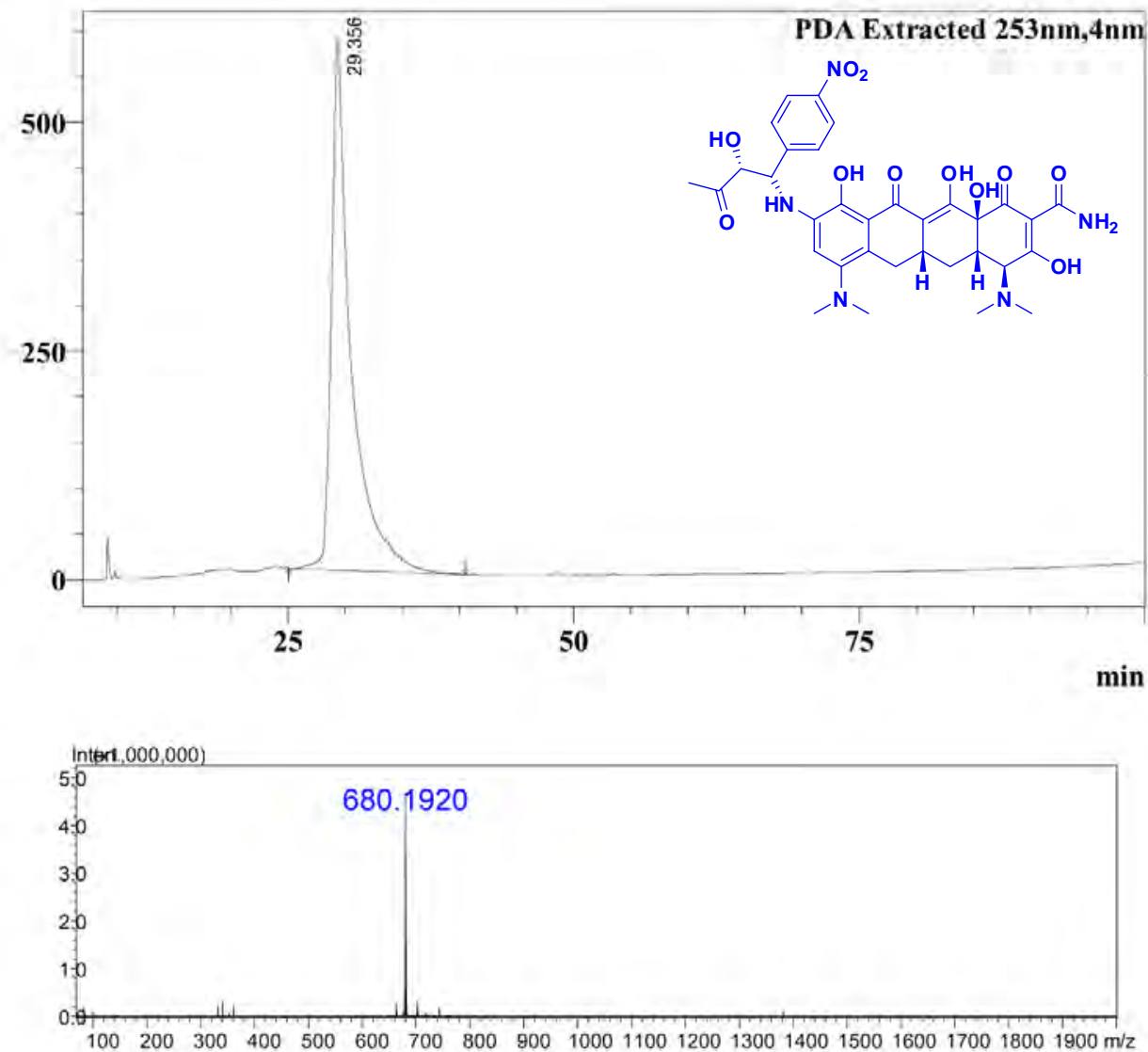
LC-MS of (4S,4aS,5aR,12aS)-9-((1S,2R)-1-(4-butoxyphenyl)-2-hydroxy-3-oxobutylamino)4,7 bis(dimethylamino)-3,10,12,12a-tetrahydroxy-1,11-dioxo-1,4,4a,5,5a,6,11,12aoctahydrotetracene-2-carboxamide (2b)

14/10/2015 15:29:14 Page 1
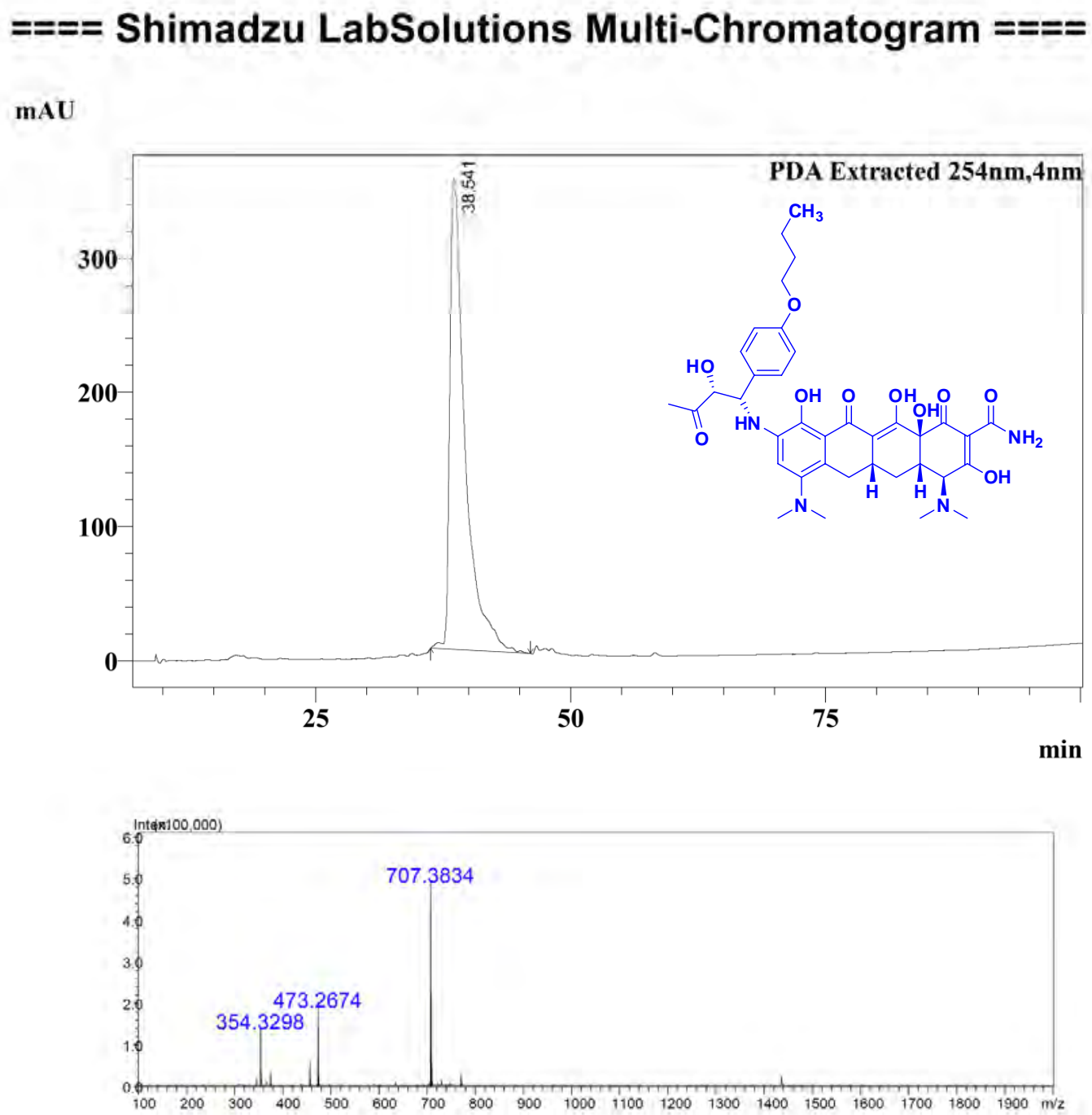
LC-MS of (4S,4aS,5aR,12aS)-4,7-bis(dimethylamino)-3,10,12,12a-tetrahydroxy-9-((1S,2R)2-hydroxy-3-oxo-1-o-tolylbutylamino)-1,11-dioxo-1,4,4a,5,5a,6,11,12a-octahydrotetracene2-carboxamide (2c)
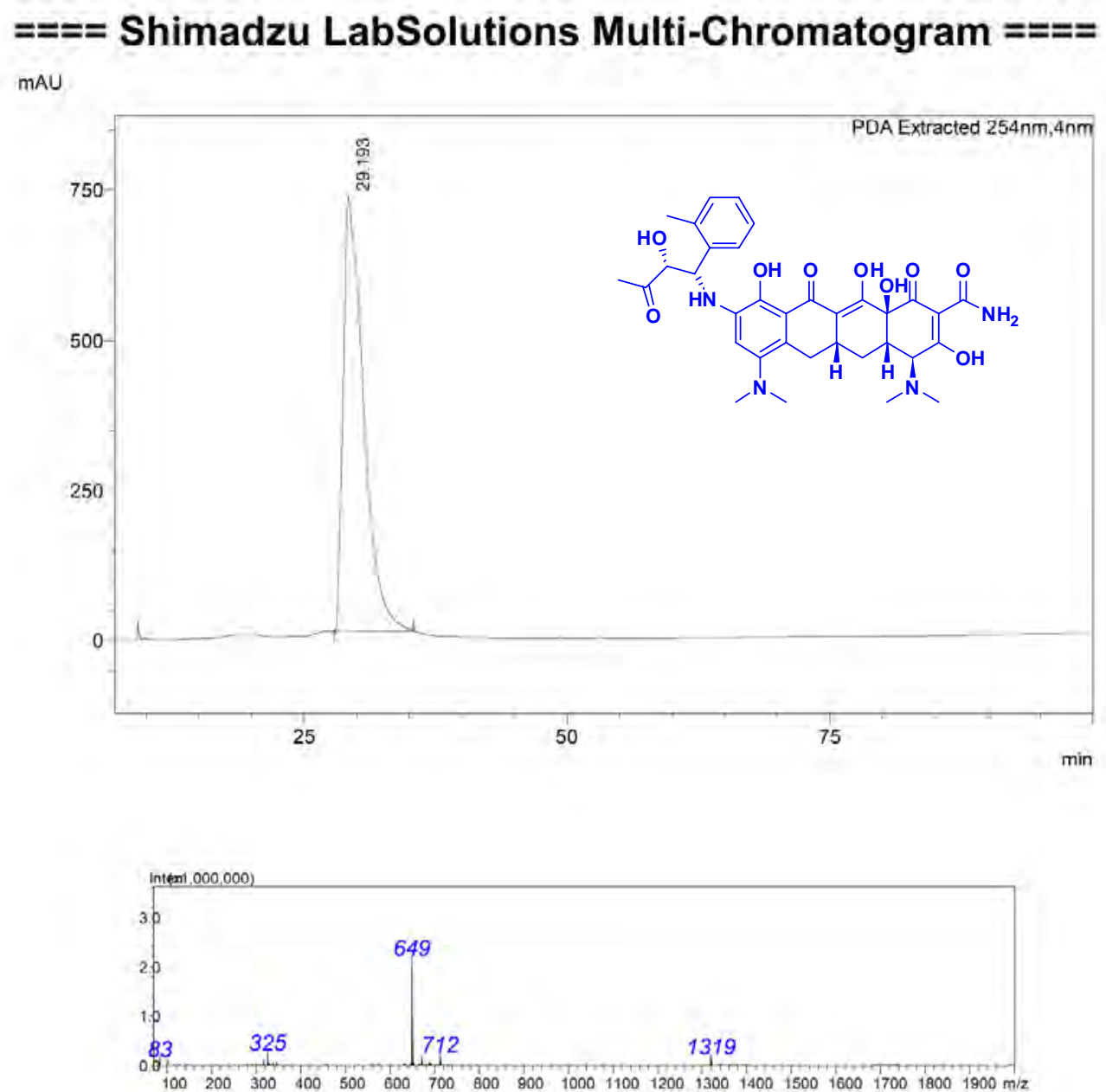
LC-MS SPECTRUM of (4S,4aS,5aR,12aS)-4,7-bis(dimethylamino)-9-((1S,2R)-1-(4fluorophenyl)-2-hydroxy-3-oxobutylamino)-3,10,12,12a-tetrahydroxy-1,11-dioxo1,4,4a,5,5a,6,11,12a-octahydrotetracene-2-carboxamide (2d)

==== Shimadzu LabSolutions Multi-Chromatogram ====

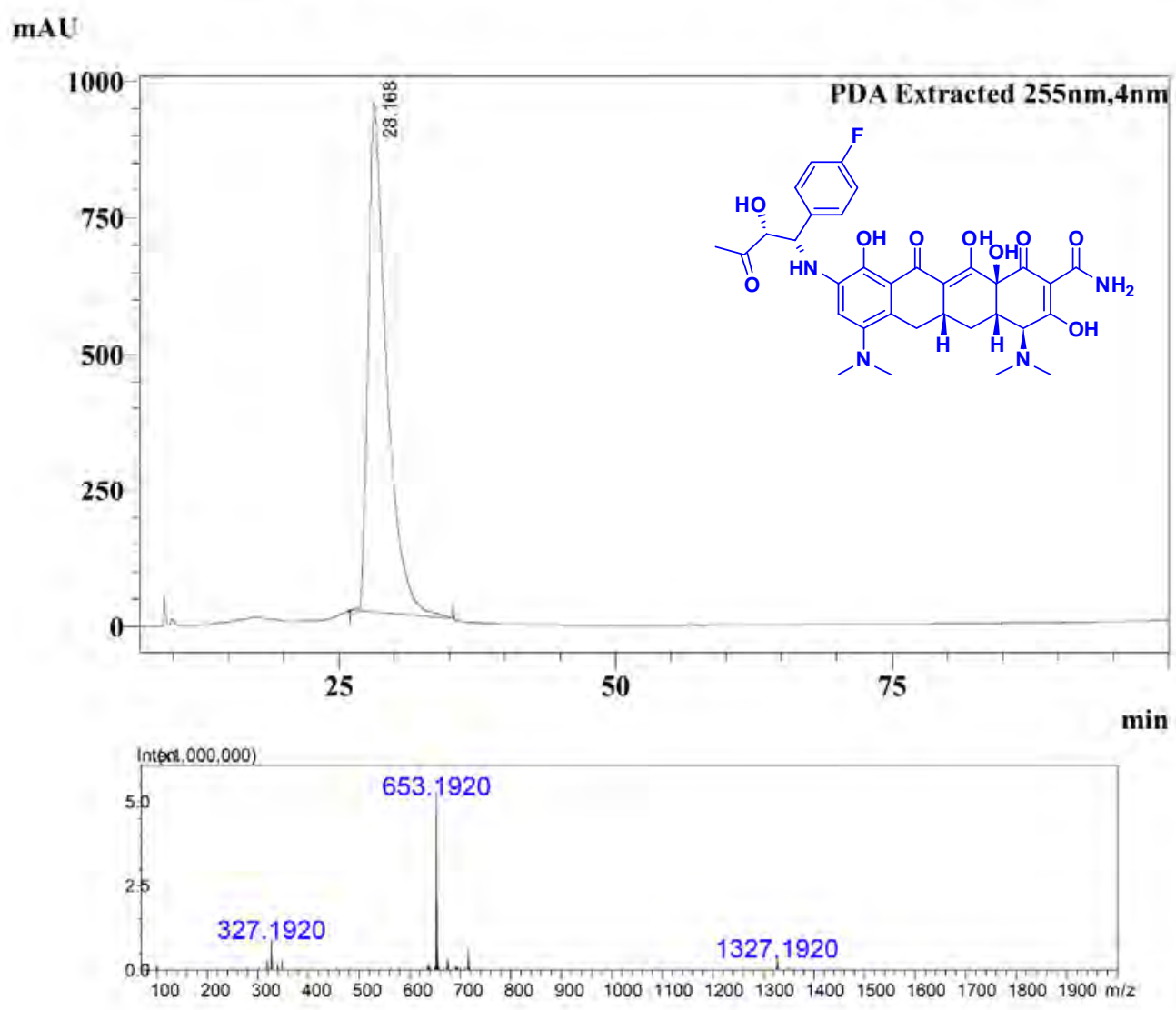


LC-MS of (4S,4aS,5aR,12aS)-4,7-bis(dimethylamino)-3,10,12,12a-tetrahydroxy-9-((1S,2R)2-hydroxy-1-(naphthalen-2-yl)-3-oxobutylamino)-1,11-dioxo-1,4,4a,5,5a,6,11,12aoctahydrotetracene-2-carboxamide (2e)

==== Shimadzu LabSolutions Multi-Chromatogram ==== mAU
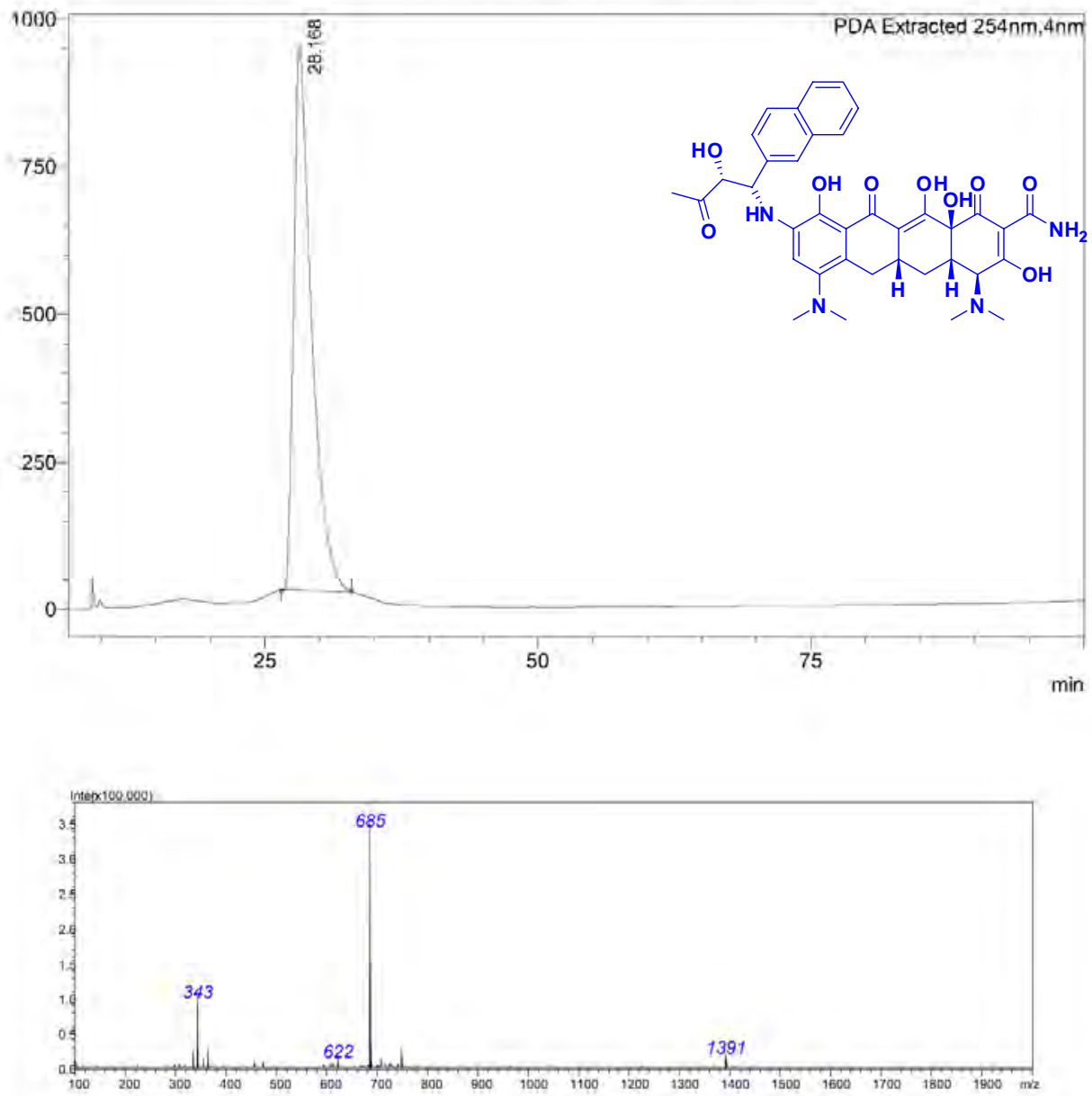
LC-MS of (4S,4aS,5aR,12aS)-4,7-bis(dimethylamino)-3,10,12,12a-tetrahydroxy-9-((1R,2R)2-hydroxy-1-(5-nitrofuran-2-yl)-3-oxobutylamino)-1,11-dioxo-1,4,4a,5,5a,6,11,12 octahydrotetracene-2-carboxamide (2f)

\section{=ニ== Shimadzu LabSolutions Multi-Chromatogram ==== mAU}
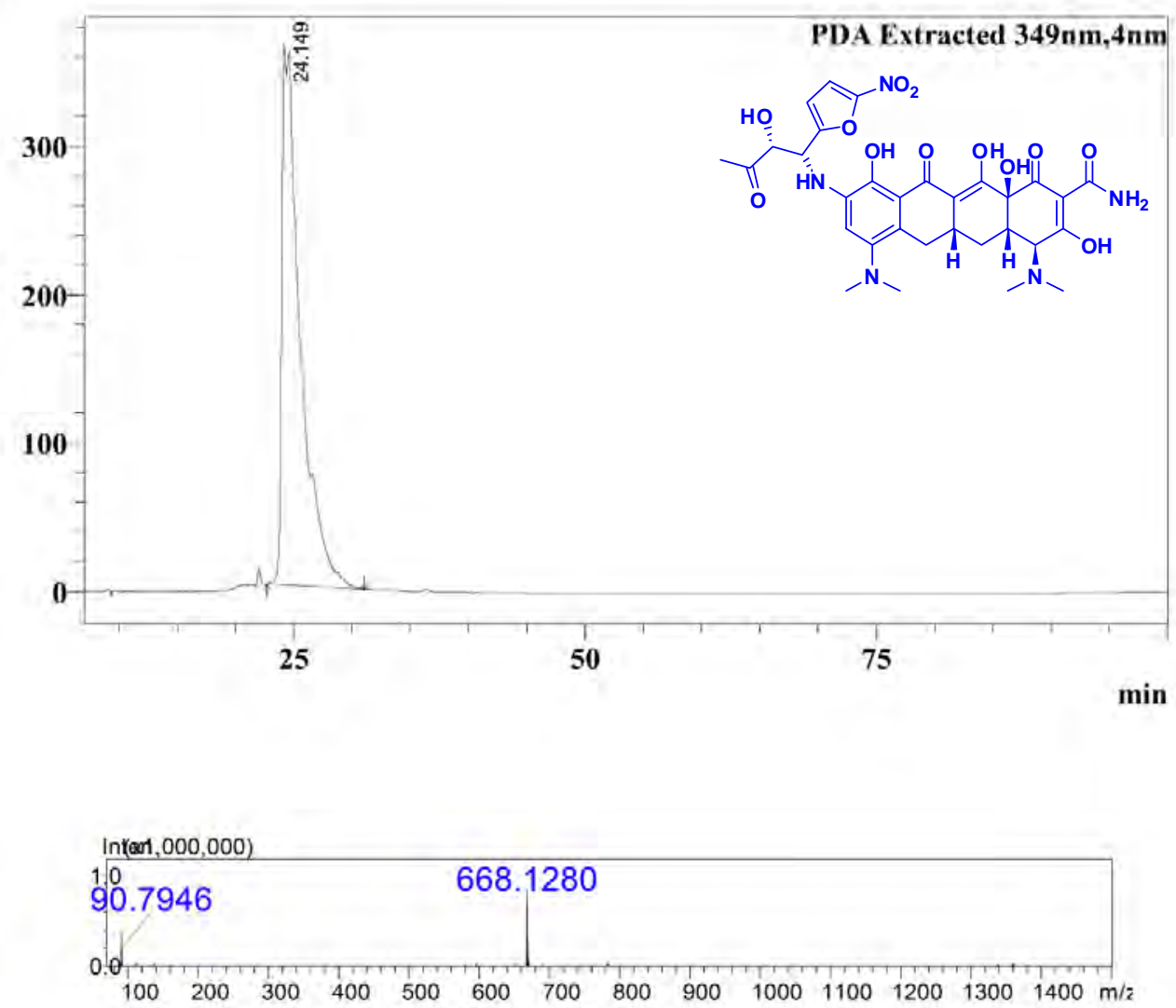
LC-MS of (4S,4aS,5aR,12aS)-4,7-bis(dimethylamino)-9-((1R,2R)-1-(furan-2-yl)-2-hydroxy3-oxobutylamino)-3,10,12,12a-tetrahydroxy-1,11-dioxo-1,4,4a,5,5a,6,11,12aoctahydrotetracene-2-carboxamide (2g)
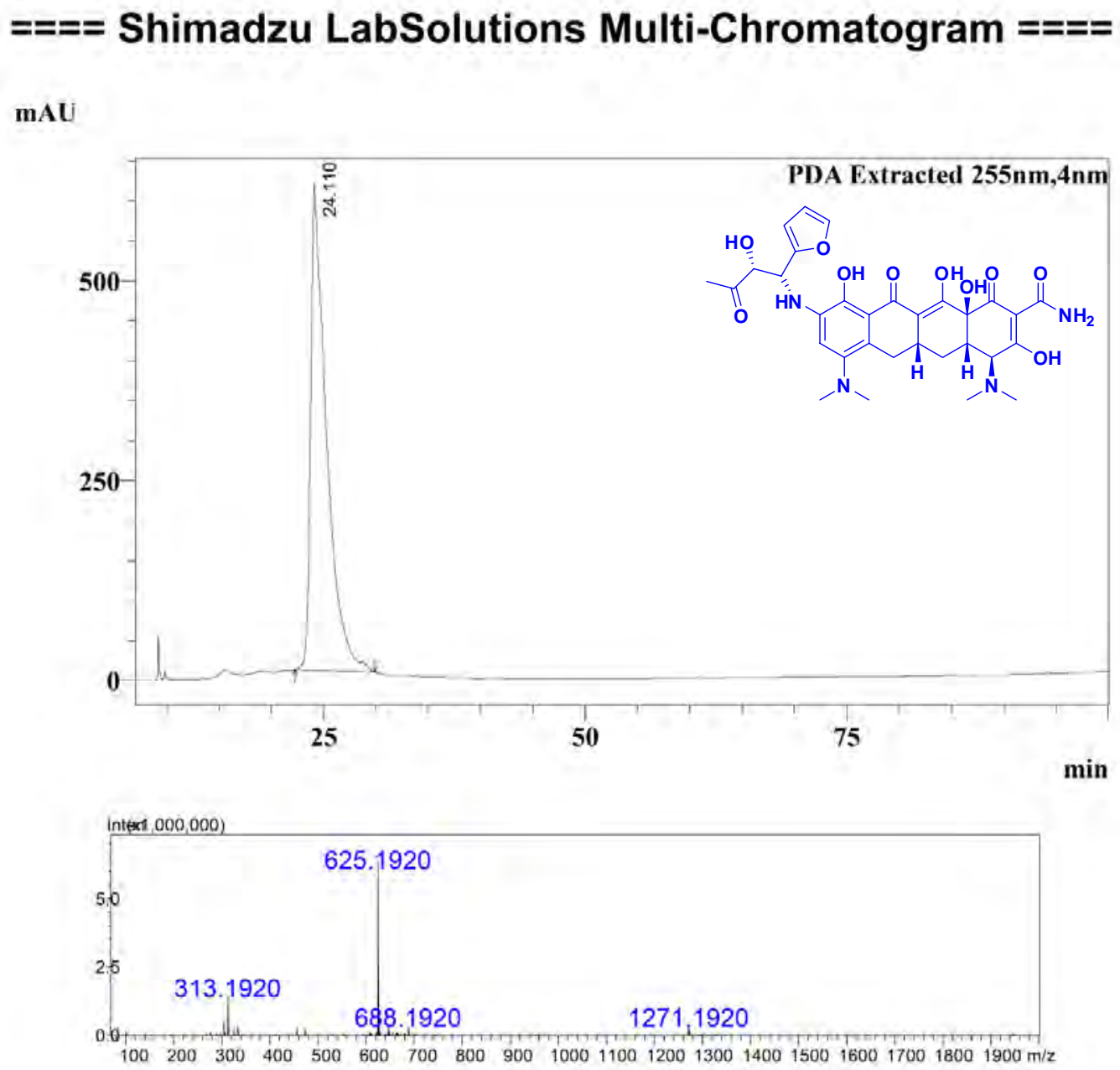
LC-MS of (4S,4aS,5aR,12aS)-9-(1-(5-bromofuran-2-yl)-2-hydroxy-3-oxobutylamino)-4,7bis (dimethylamino)-3,10,12,12a-tetrahydroxy-1,11-dioxo-1,4,4a,5,5a,6,11,12aoctahydrotetracene-2-carboxamide (2h)

14/10/2015 15:15:01 Page 1

==== Shimadzu LabSolutions Multi-Chromatogram ==== mAU
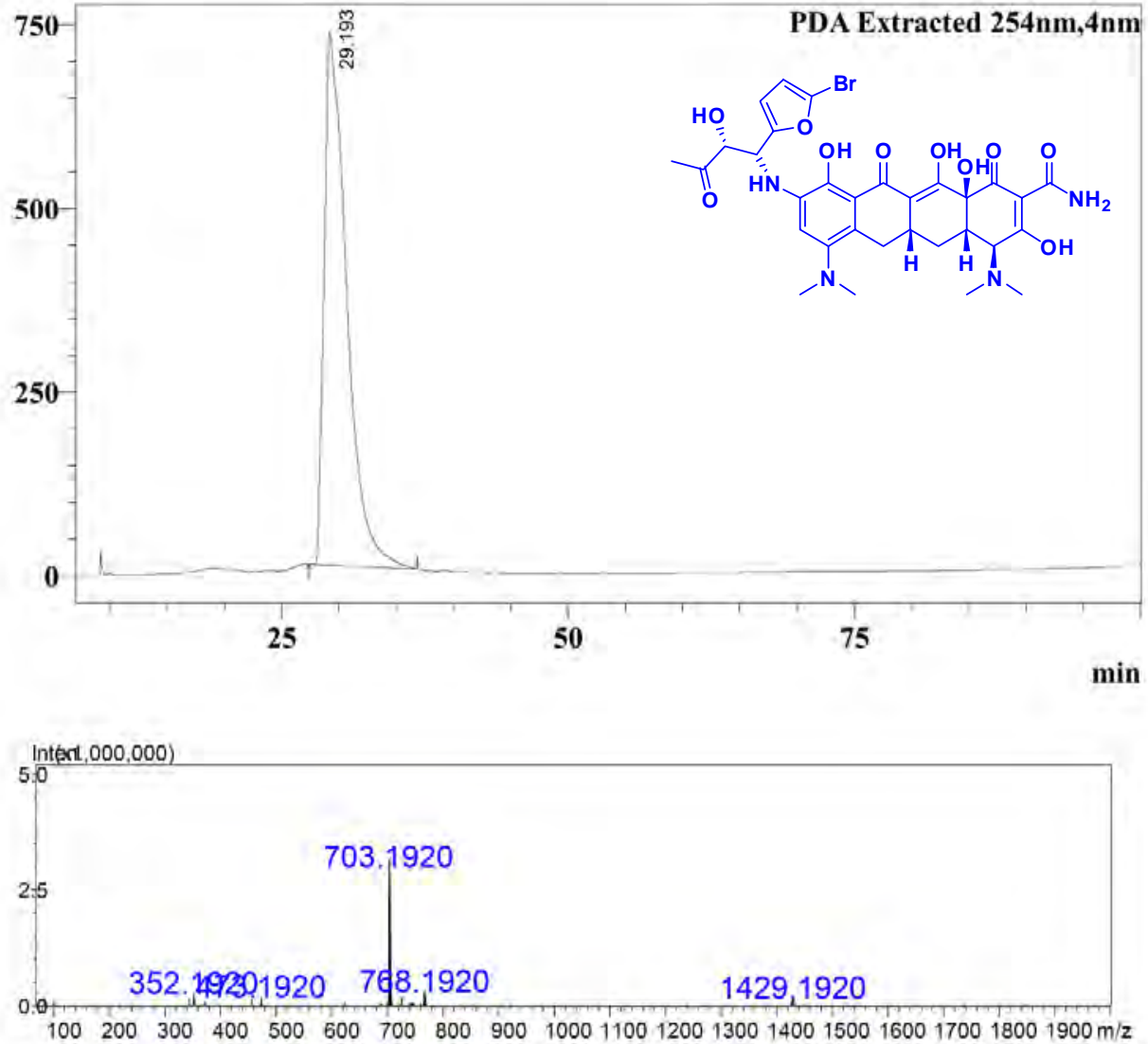


\section{Copies of NMR spectra for products}

${ }^{1} \mathrm{H}$ NMR of (4S,4aS,5aR,12aS)-4,7-bis(dimethylamino)-3,10,12,12a-tetrahydroxy-9-(2-hydroxy-3-oxo-1-phenylbutylamino)1,11-dioxo-1,4,4a,5,5a,6,11,12a-octahydrotetracene-2-carboxamide (1a)

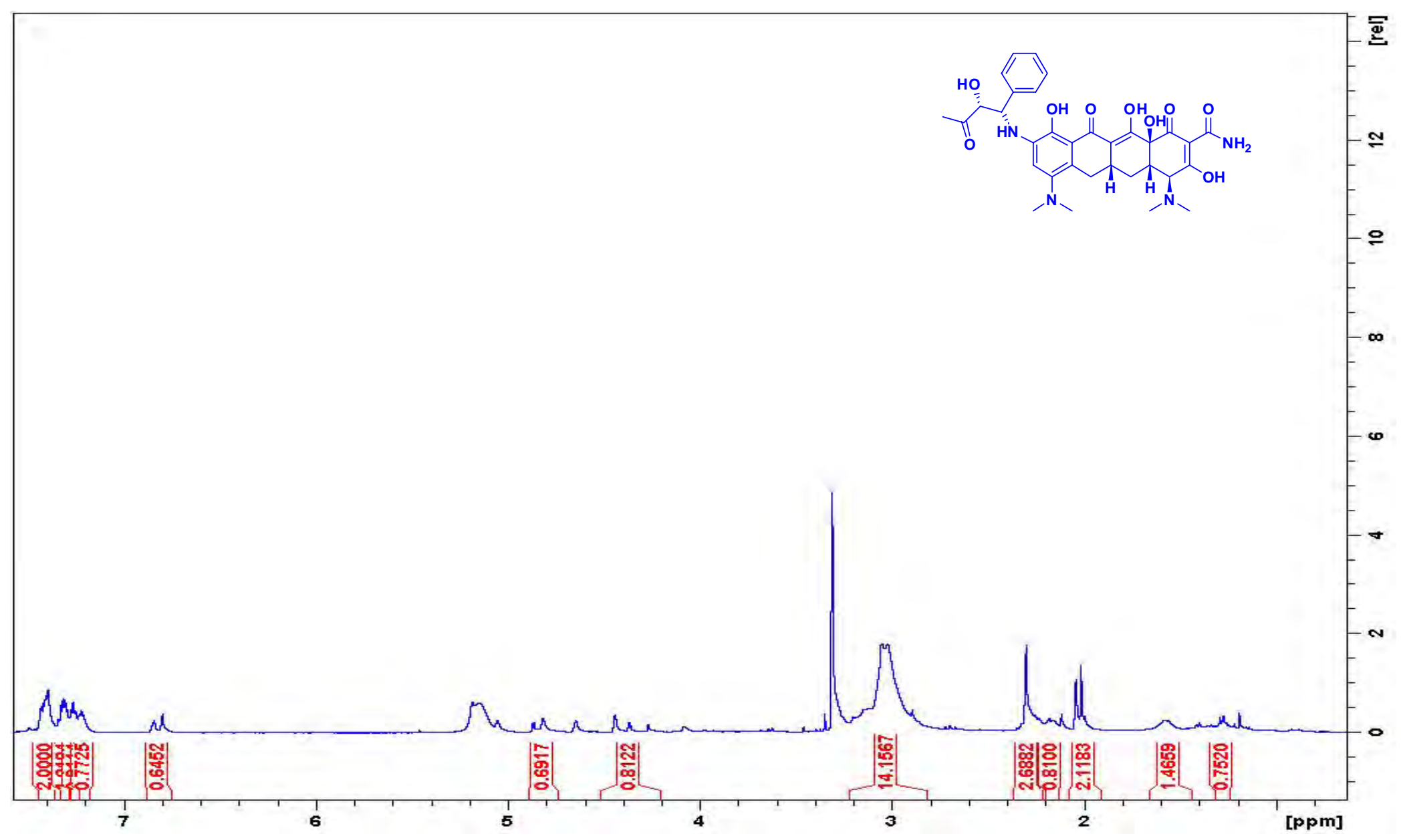


${ }^{1} \mathrm{H}$ NMR of (4S,4aS,5aR,12aS)-4,7-bis(dimethylamino)-3,10,12,12a-tetrahydroxy-9-(2-methyl-3-oxo-1-phenylbutylamino)-1,11dioxo-1,4,4a,5,5a,6,11,12a-octahydrotetracene-2-carboxamide (1b)

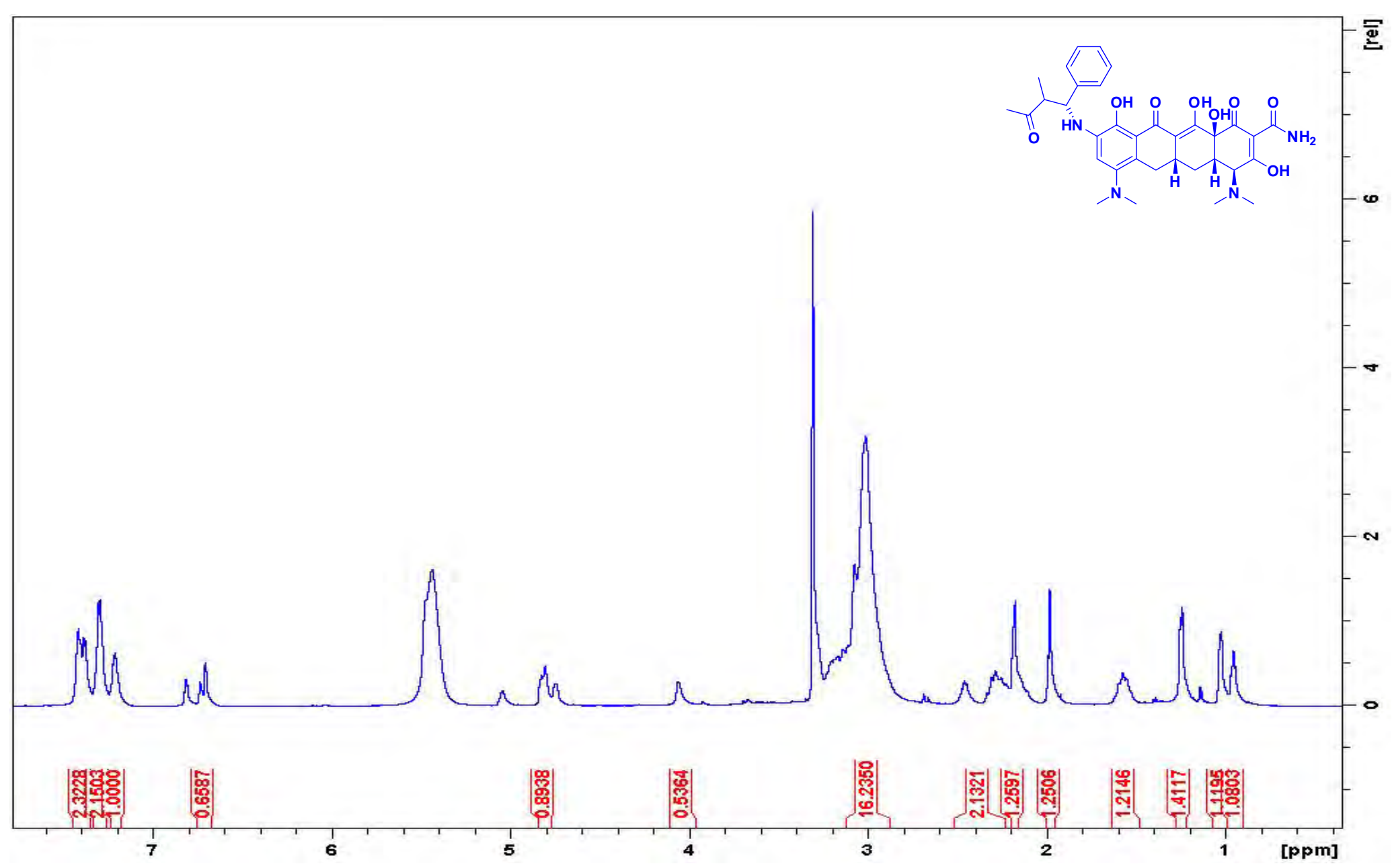


${ }^{1}$ H NMR of (4S,4aS,5aR,12aS)-4,7-bis(dimethylamino)-3,10,12,12a-tetrahydroxy-1,11-dioxo-9-(3-oxo-1-phenylbutylamino)1,4,4a,5,5a,6,11,12a-octahydrotetracene-2-carboxamide (1c)

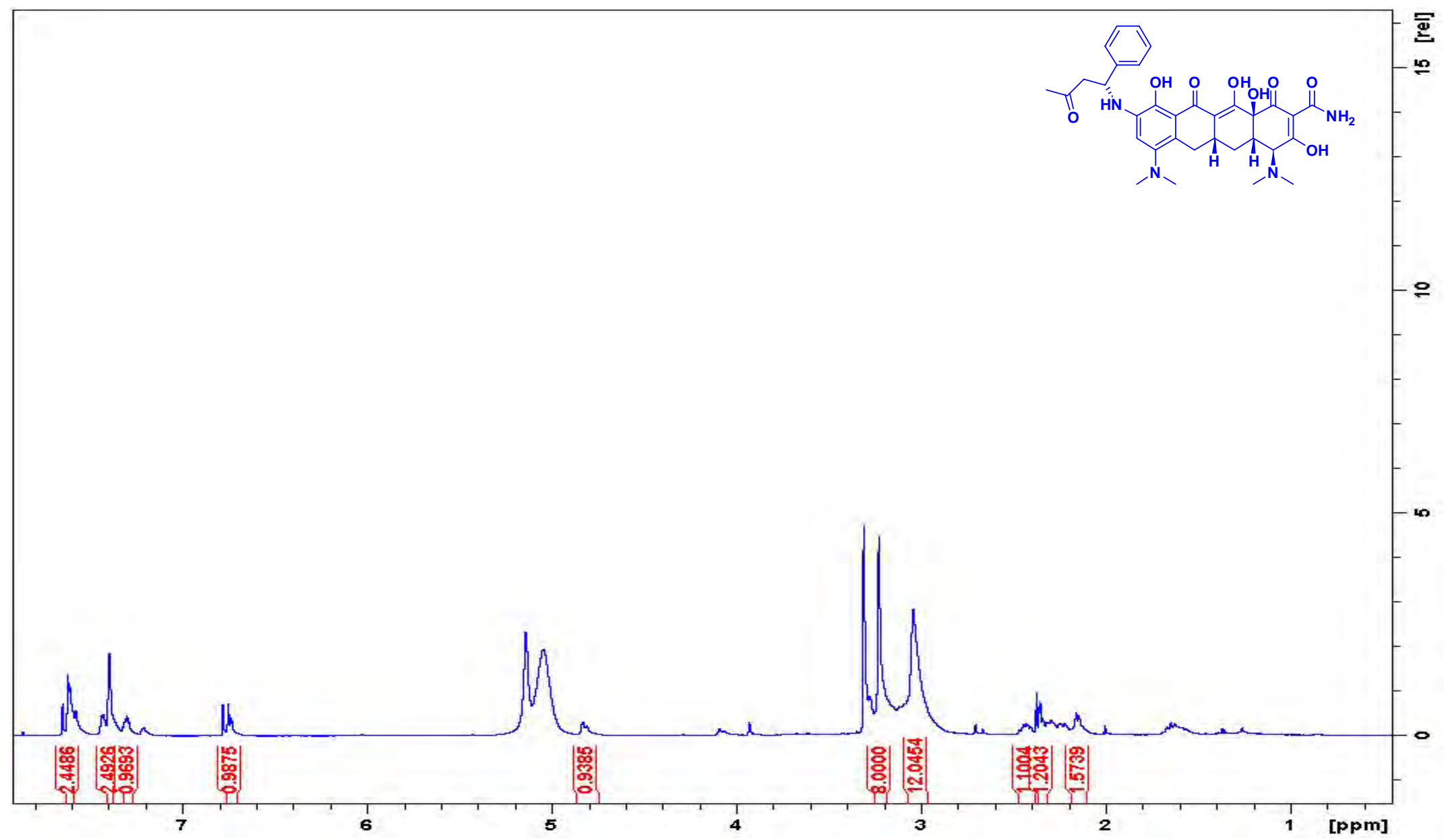


${ }^{1} \mathrm{H}$ NMR of (4S,4aS,5aR,12aS)-4,7-bis(dimethylamino)-3,10,12,12a-tetrahydroxy-1,11-dioxo-9-((1R)-(2oxocyclohexyl)(phenyl)methylamino)-1,4,4a,5,5a,6,11,12a-octahydrotetracene-2-carboxamide (1d)

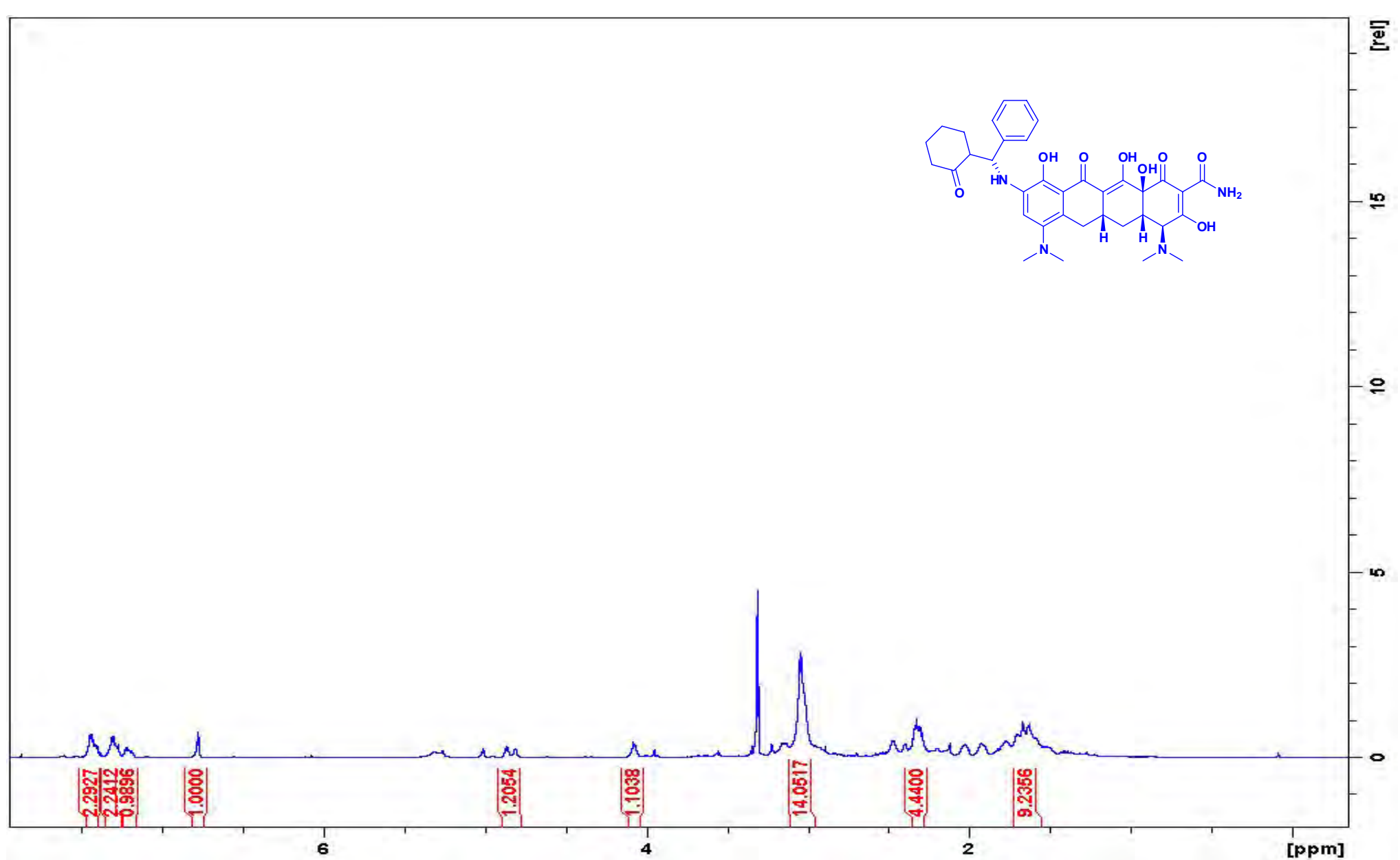


${ }^{1}$ H NMR of (4S,4aS,5aR,12aS)-4,7-bis(dimethylamino)-3,10,12,12a-tetrahydroxy-9-((1S,2R)-2-hydroxy-1-(4-nitrophenyl)-3oxobutylamino)-1,11-dioxo-1,4,4a,5,5a,6,11,12a-octahydrotetracene-2-carboxamide (2a)

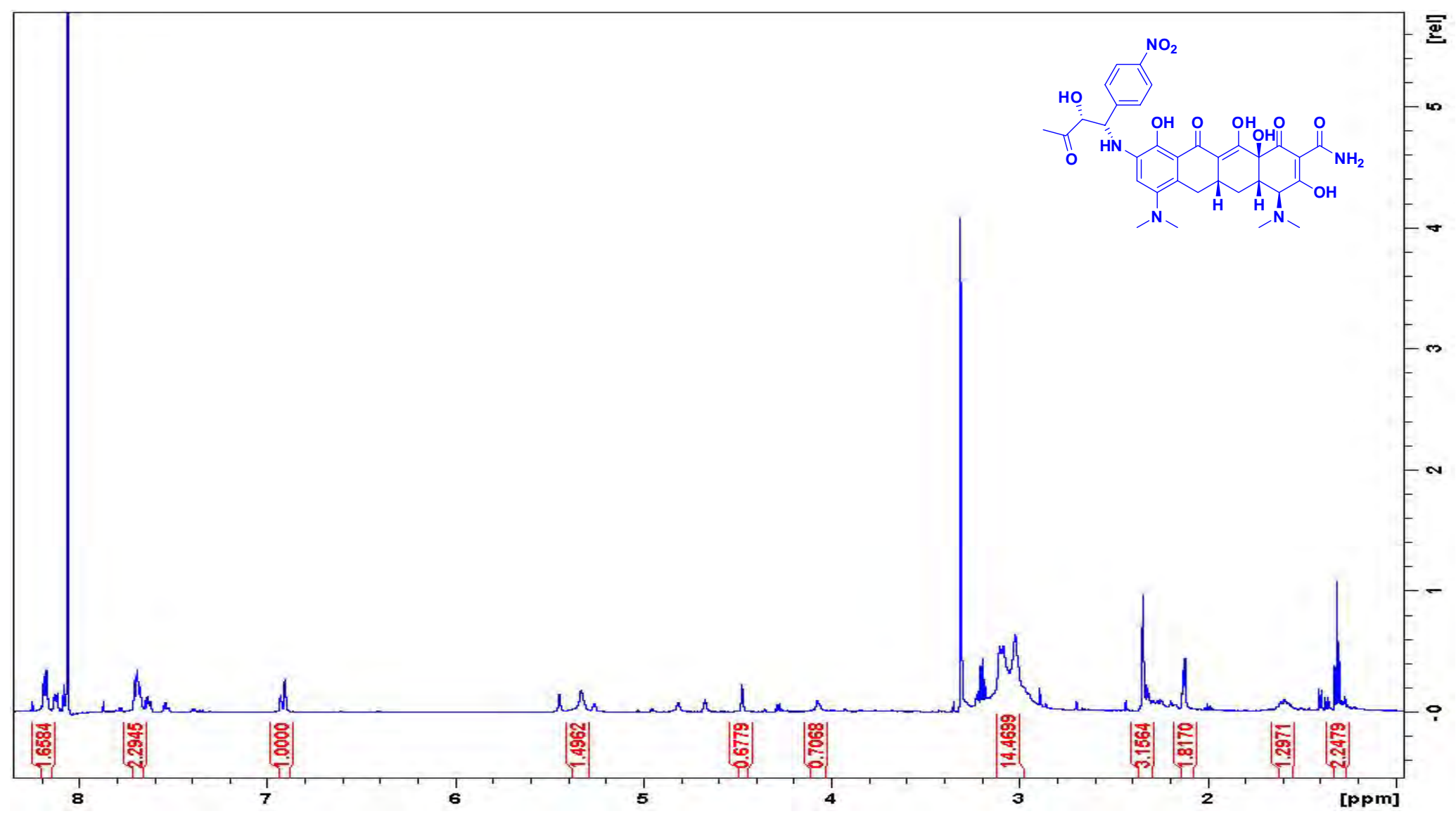


${ }^{1}$ H NMR of (4S,4aS,5aR,12aS)-9-((1S,2R)-1-(4-butoxyphenyl)-2-hydroxy-3-oxobutylamino)-4,7 bis(dimethylamino)3,10,12,12a-tetrahydroxy-1,11-dioxo-1,4,4a,5,5a,6,11,12a-octahydrotetracene-2-carboxamide (2b)

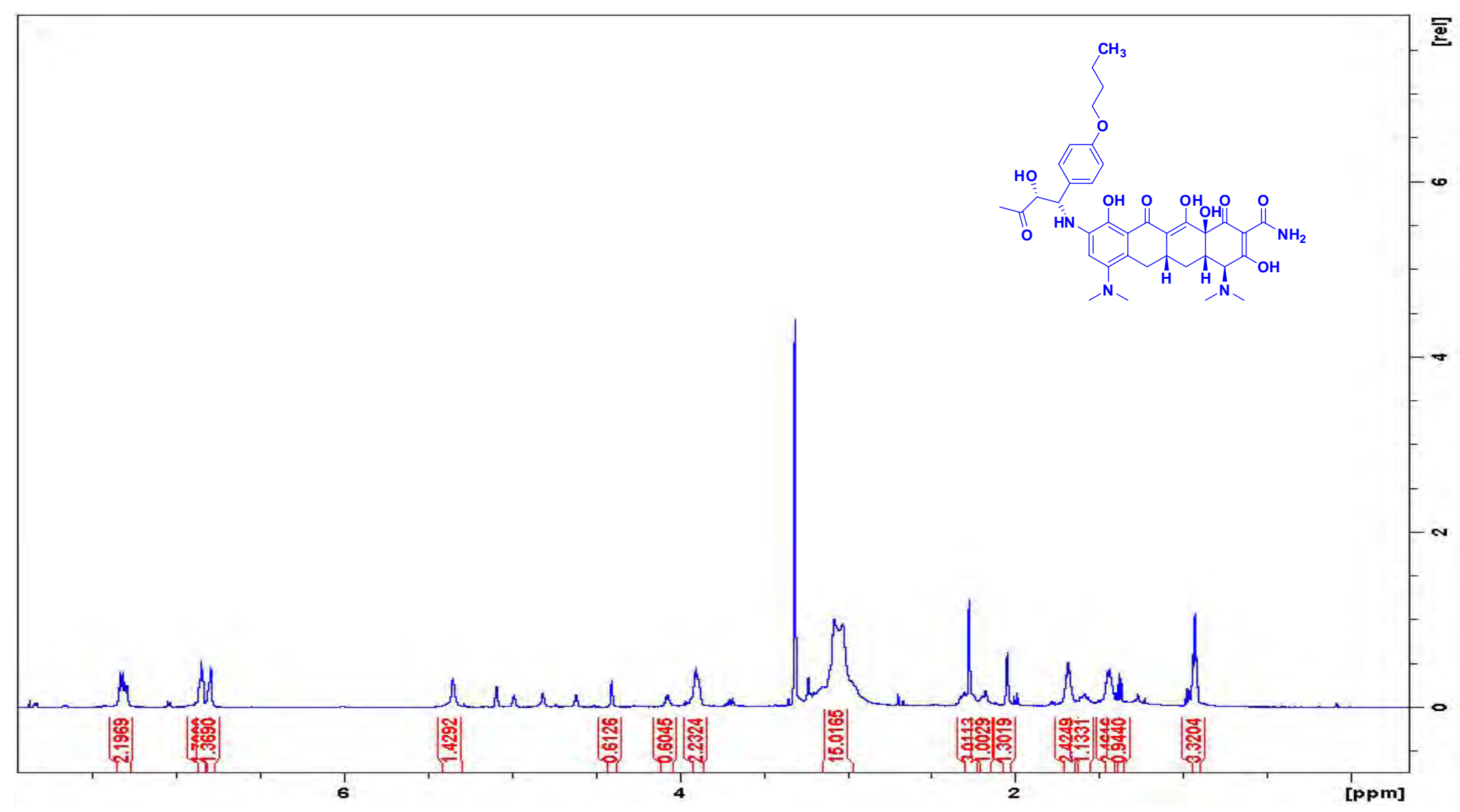


${ }^{1} \mathrm{H}$ NMR of (4S,4aS,5aR,12aS)-4,7-bis(dimethylamino)-3,10,12,12a-tetrahydroxy-9-((1S,2R)-2-hydroxy-3-oxo-1-otolylbutylamino)-1,11-dioxo-1,4,4a,5,5a,6,11,12a-octahydrotetracene-2-carboxamide (2c)

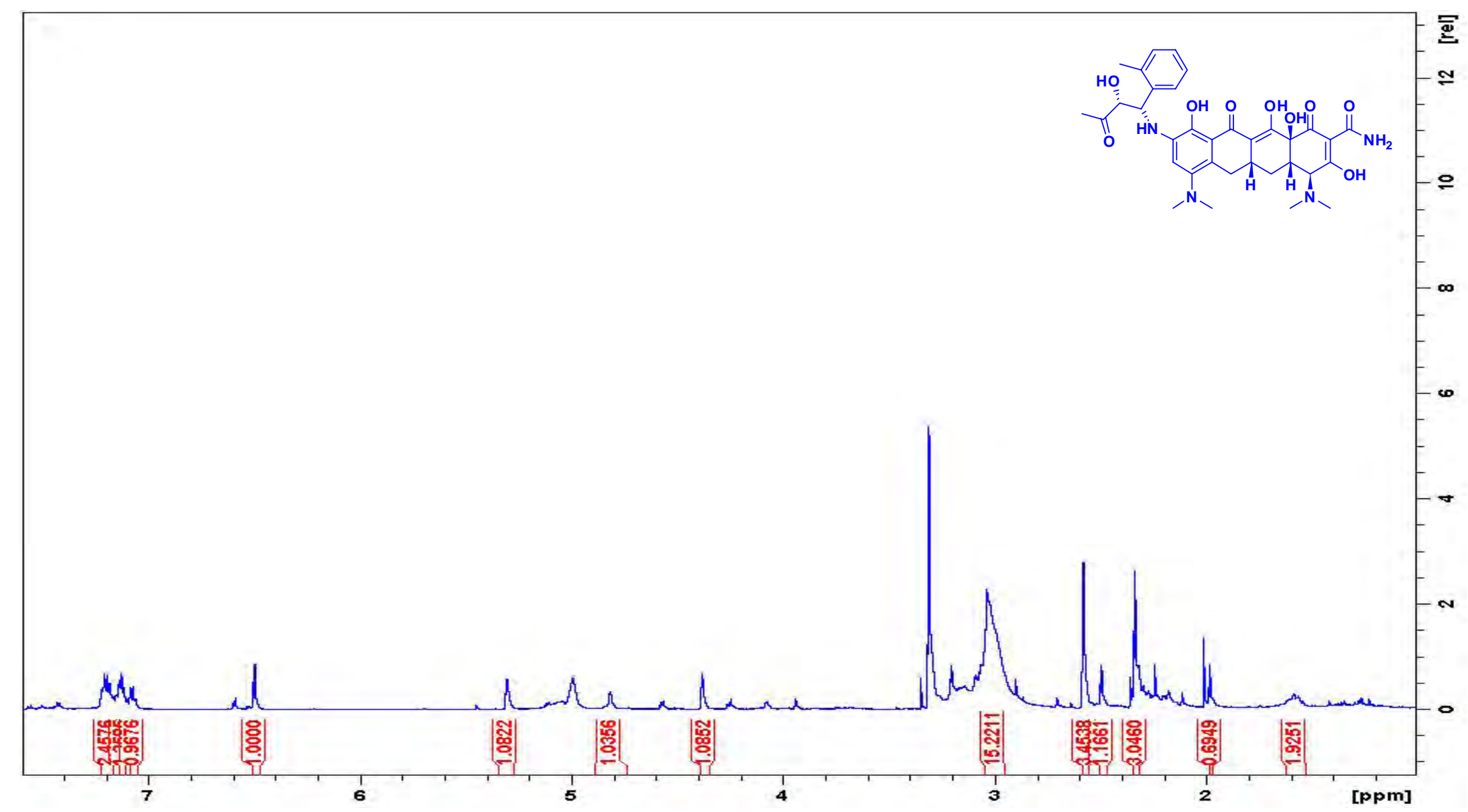


${ }^{1} \mathrm{H}$ NMR of (4S,4aS,5aR,12aS)-4,7-bis(dimethylamino)-9-((1S,2R)-1-(4-fluorophenyl)-2-hydroxy-3-oxobutylamino)3,10,12,12a-tetrahydroxy-1,11-dioxo-1,4,4a,5,5a,6,11,12a-octahydrotetracene-2-carboxamide (2d)

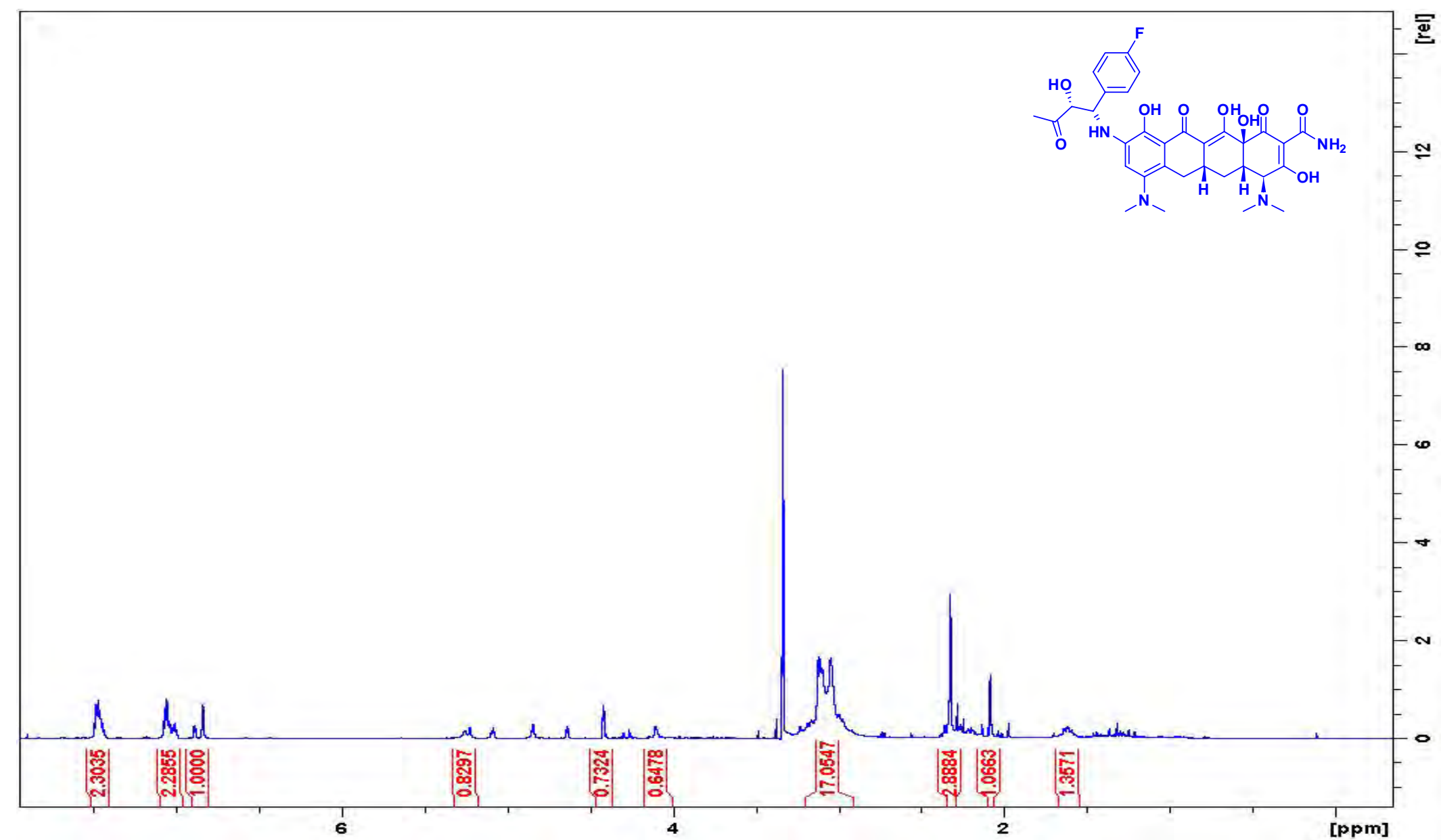


${ }^{1}$ H NMR of (4S,4aS,5aR,12aS)-4,7-bis(dimethylamino)-3,10,12,12a-tetrahydroxy-9-((1S,2R)-2-hydroxy-1-(naphthalen-2-yl)-3oxobutylamino)-1,11-dioxo-1,4,4a,5,5a,6,11,12a-octahydrotetracene-2-carboxamide (2e)

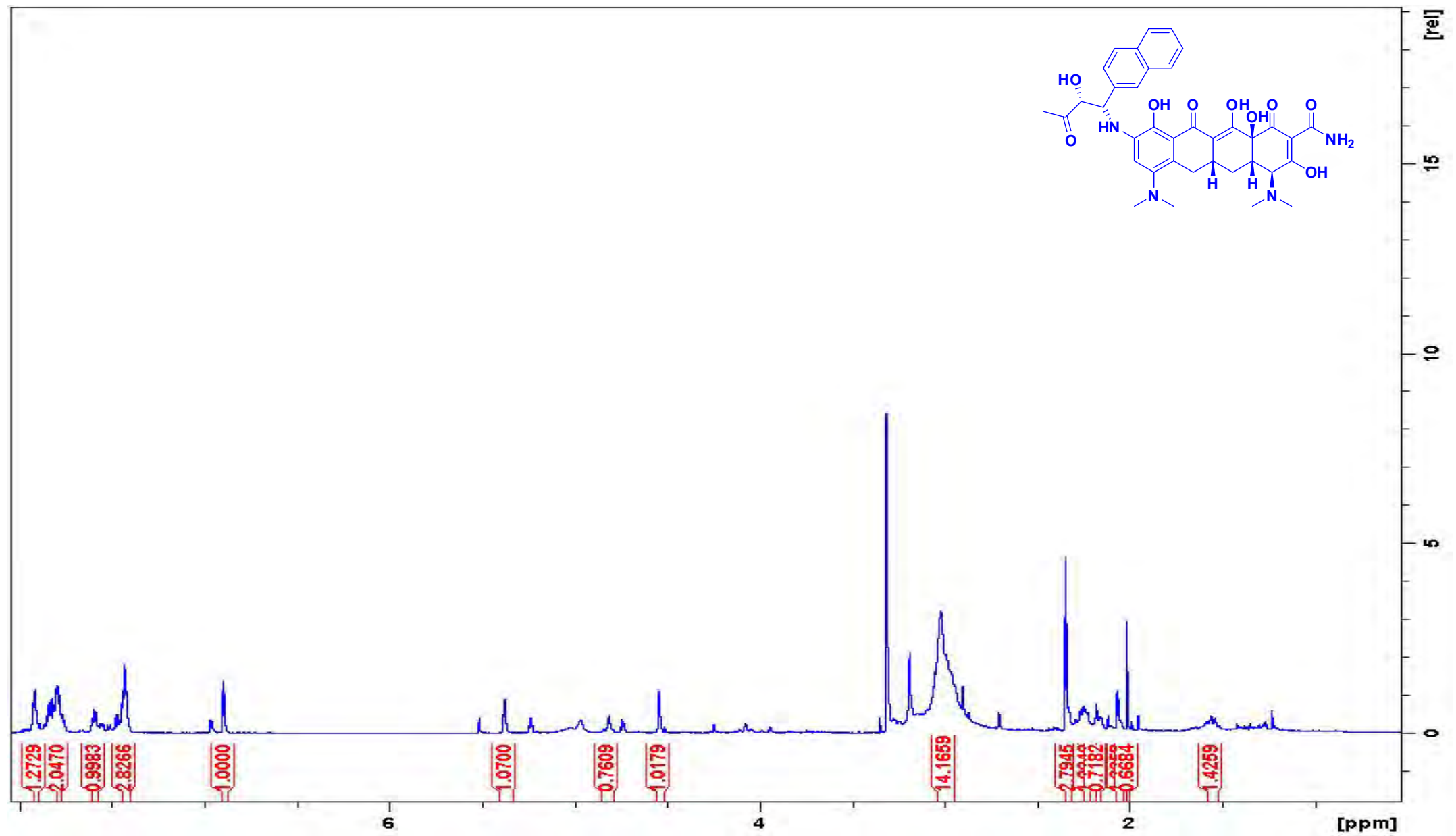


${ }^{1} \mathrm{H}$ NMR of (4S,4aS,5aR,12aS)-4,7-bis(dimethylamino)-3,10,12,12a-tetrahydroxy-9-((1R,2R)-2-hydroxy-1-(5-nitrofuran-2-yl)3-oxobutylamino)-1,11-dioxo-1,4,4a,5,5a,6,11,12a-octahydrotetracene-2-carboxamide (2f)

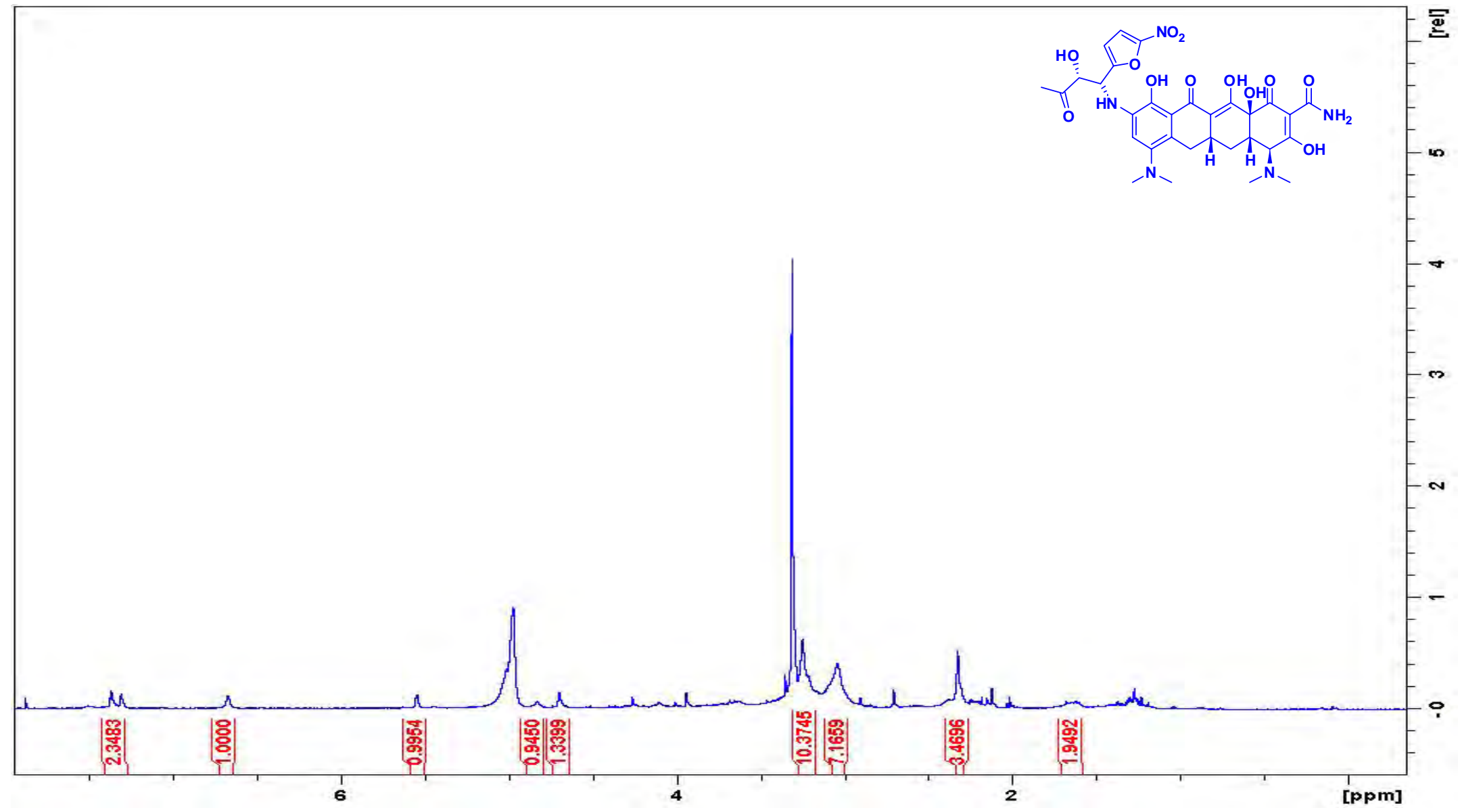


${ }^{1} \mathrm{H}$ NMR of (4S,4aS,5aR,12aS)-4,7-bis(dimethylamino)-9-((1R,2R)-1-(furan-2-yl)-2-hydroxy-3-oxobutylamino)-3,10,12,12atetrahydroxy-1,11-dioxo-1,4,4a,5,5a,6,11,12a-octahydrotetracene-2-carboxamide (2g)

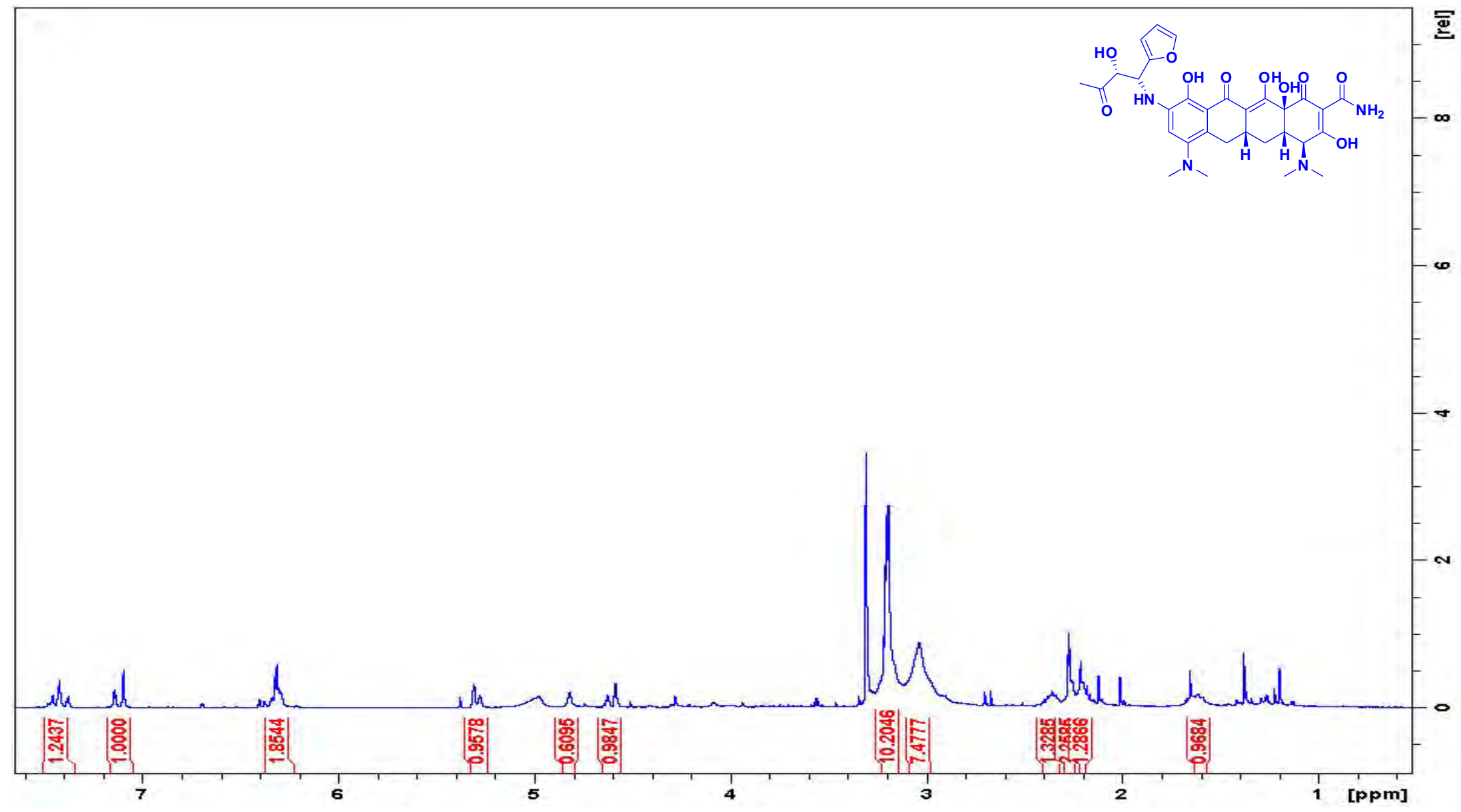


${ }^{1} \mathrm{H}$ NMR of (4S,4aS,5aR,12aS)-9-(1-(5-bromofuran-2-yl)-2-hydroxy-3-oxobutylamino)-4,7-bis (dimethylamino)-3,10,12,12atetrahydroxy-1,11-dioxo-1,4,4a,5,5a,6,11,12a-octahydrotetracene-2-carboxamide (2h)

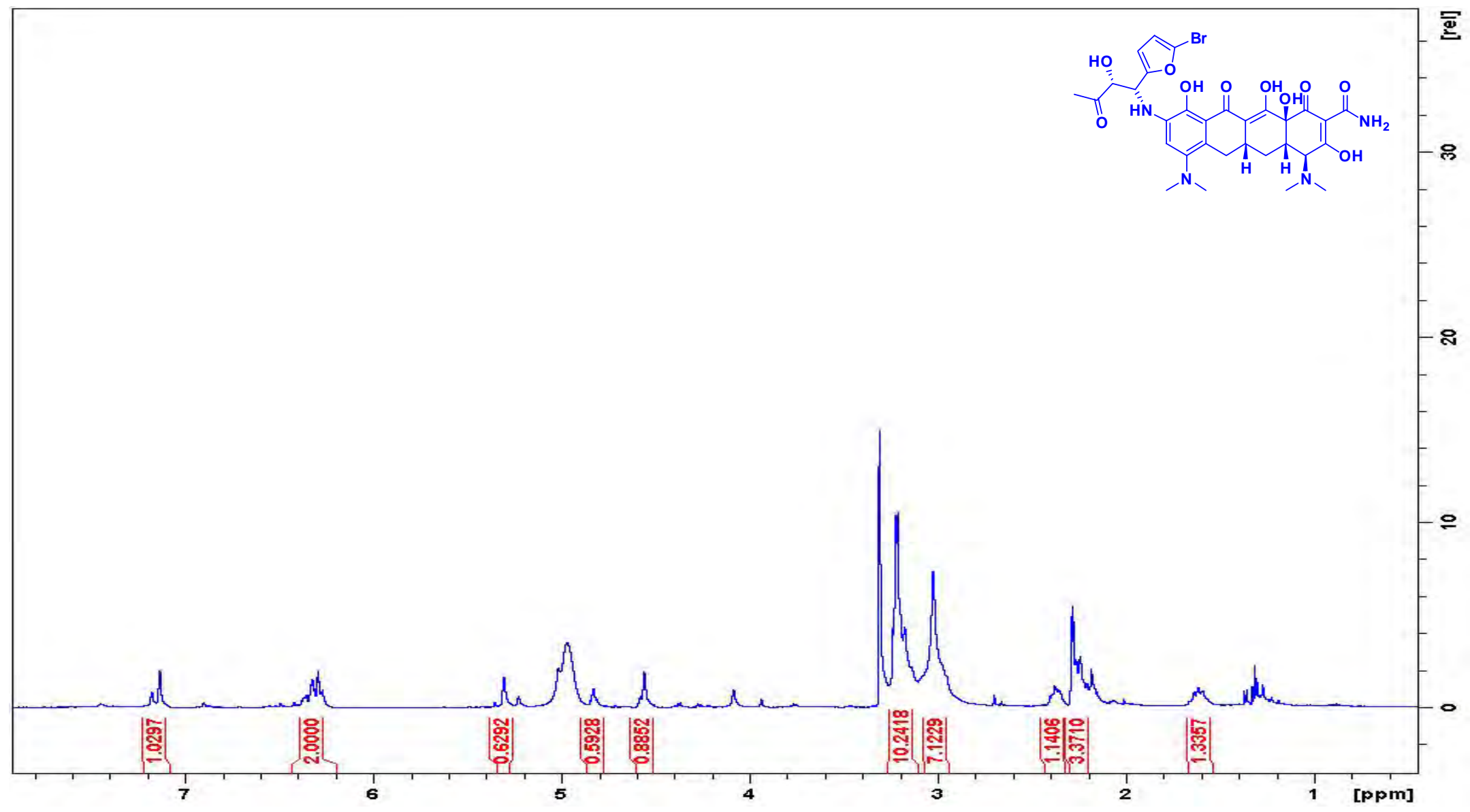


Organocatalyzed Mannich Reactions on Minocycline: Towards Novel Tetracycline Antibiotics

Tirivashe E. Chiwunze ${ }^{[a]}$, Rafiatu Azumah ${ }^{[a]}$, Melissa Ramtahal ${ }^{[a]}$, Per I. Arvidsson ${ }^{[b]}$, Sabiha Y. Essack ${ }^{[c]}$, Hendrik G. Kruger ${ }^{[a]}$, Thavendran Govender ${ }^{[a]}$, Tricia Naicker ${ }^{[a]}$

${ }^{a}$ School of Pharmacy and Pharmacology, University of KwaZulu-Natal, Westville Campus, Durban 4000, South Africa.

*e-mail: Naickert1@ukzn.ac.za>

${ }^{\mathbf{b}}$ Science for Life Laboratory, Drug Discovery and Development Platform, and Division of Translational Medicine and Chemical Biology, Department of Medical Biochemistry and Biophysics, Karolinska Institutet, Stockholm, Sweden

${ }^{\mathrm{c}}$ School of Chemistry and Physics, University of KwaZulu-Natal, Westville Campus, Durban 4000, South Africa

\section{SUPPLEMETARY DATA}




\section{Contents}

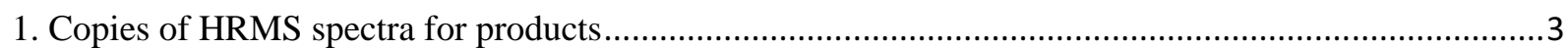

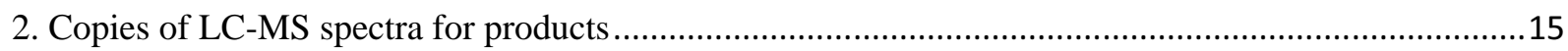

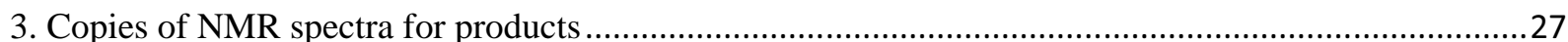




\section{Copies of HRMS spectra for products}

HRMS of (4S,4aS,5aR,12aS)-4,7-bis(dimethylamino)-3,10,12,12a-tetrahydroxy-9-(2hydroxy-3-oxo-1-phenylbutylamino)-1,11-dioxo-1,4,4a,5,5a,6,11,12a-octahydrotetracene-2carboxamide (1a)

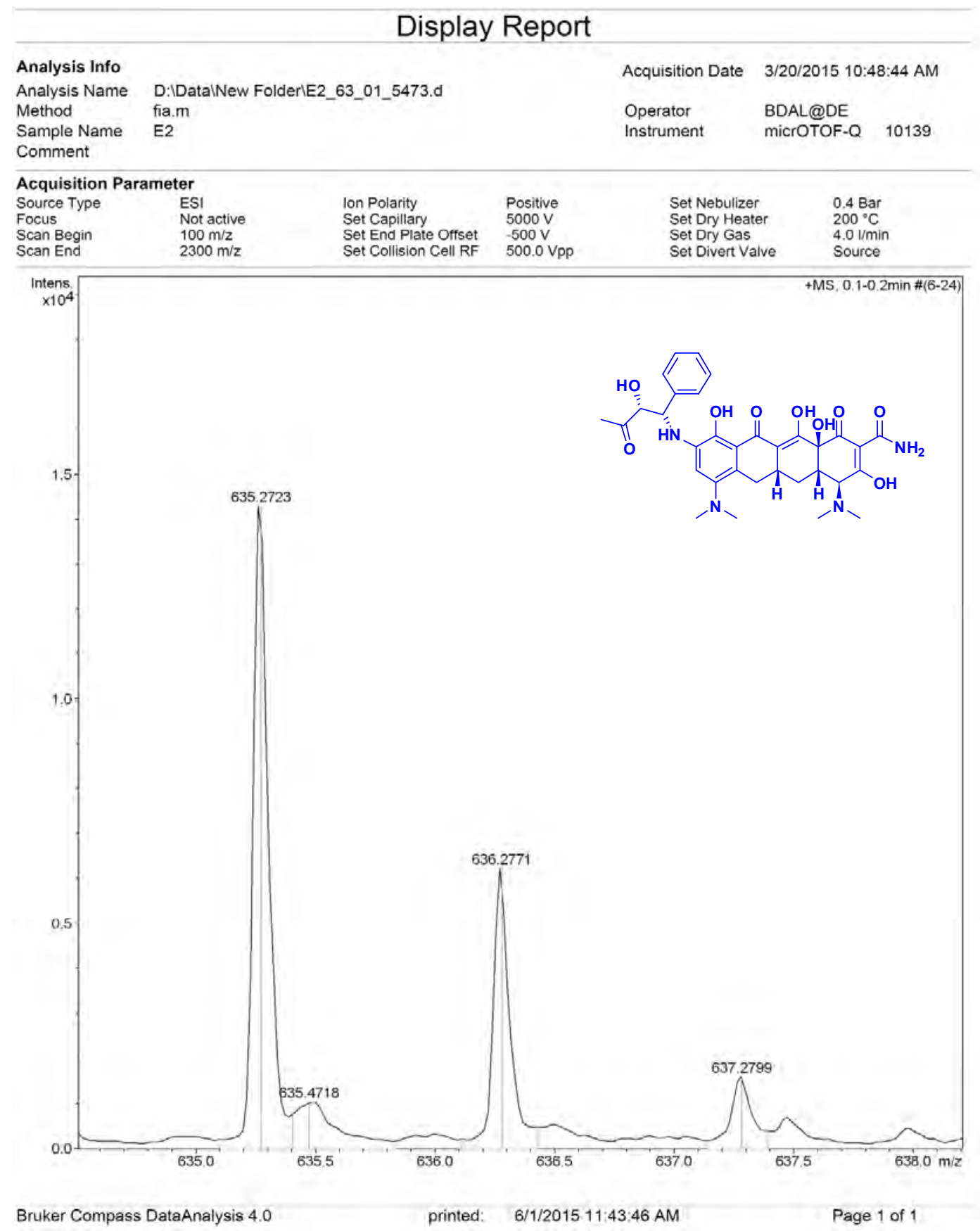


HRMS of (4S,4aS,5aR,12aS)-4,7-bis(dimethylamino)-3,10,12,12a-tetrahydroxy-9-(2methyl-3-oxo-1-phenylbutylamino)-1,11-dioxo-1,4,4a,5,5a,6,11,12a-octahydrotetracene-2carboxamide (1b)

\section{Display Report}

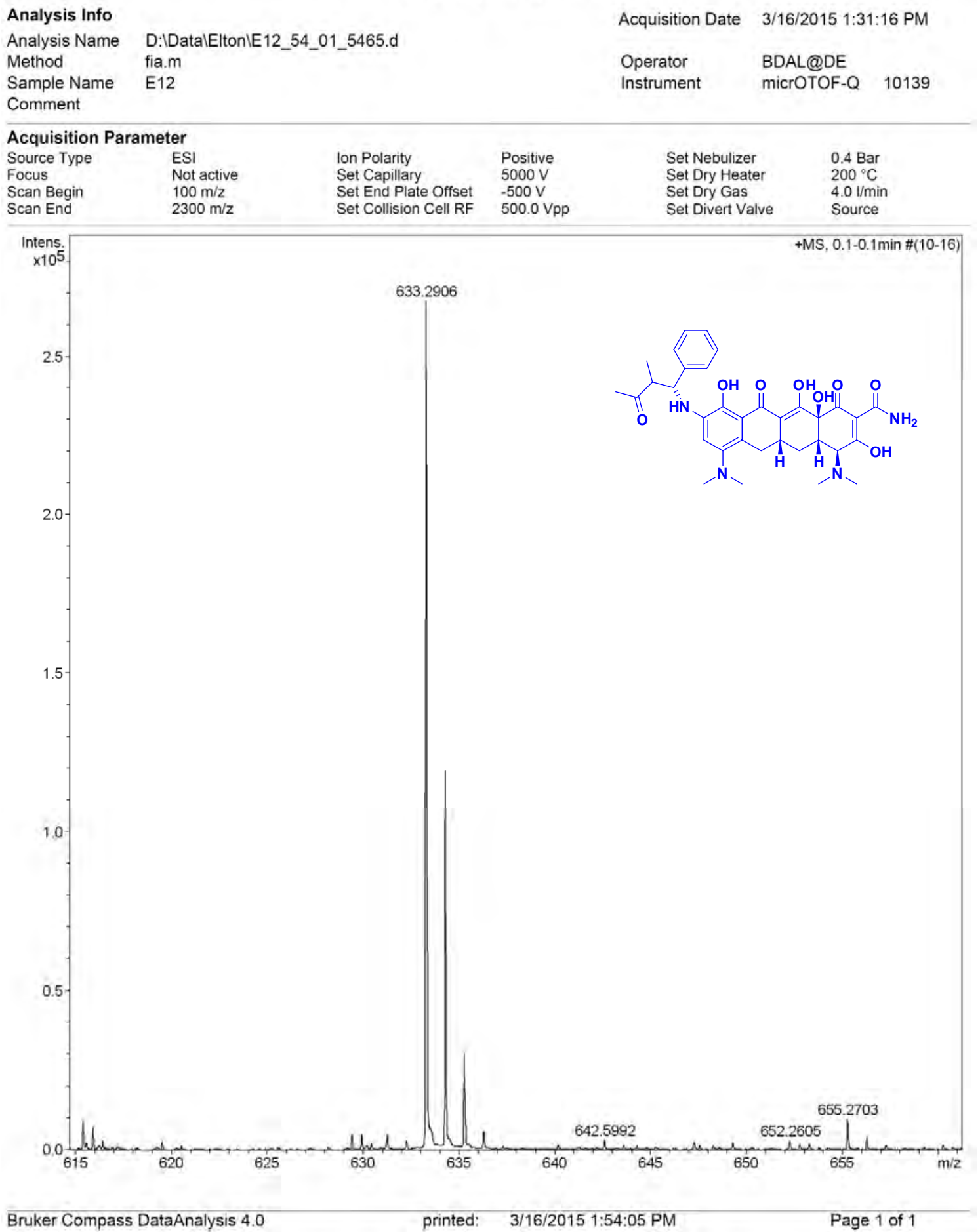


HRMS of (4S,4aS,5aR,12aS)-4,7-bis(dimethylamino)-3,10,12,12a-tetrahydroxy-1,11-dioxo-9(3-oxo-1-phenylbutylamino)-1,4,4a,5,5a,6,11,12a-octahydrotetracene-2-carboxamide (1c)

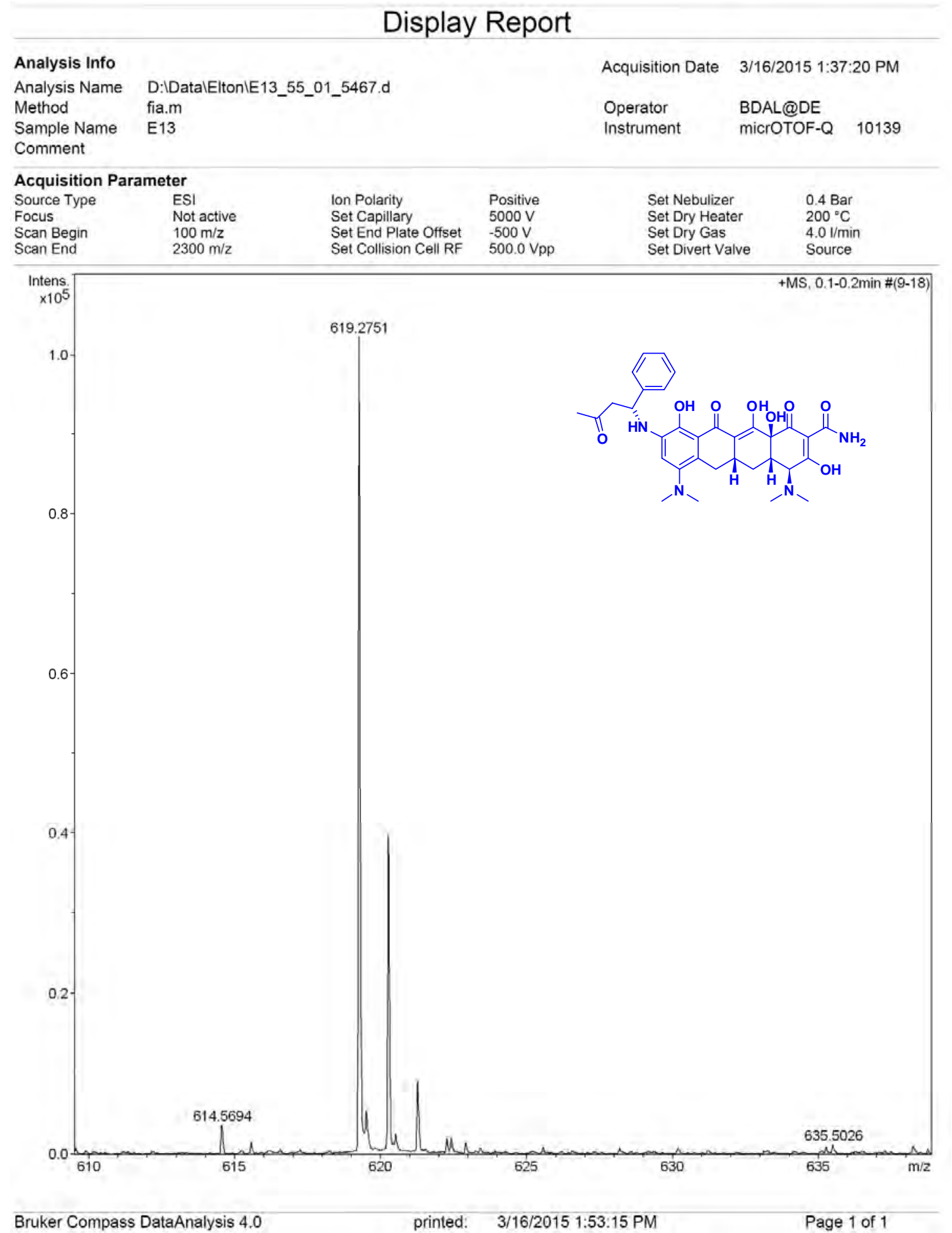


HRMS of (4S,4aS,5aR,12aS)-4,7-bis(dimethylamino)-3,10,12,12a-tetrahydroxy-1,11-dioxo-9((1R)-(2-oxocyclohexyl)(phenyl)methylamino)-1,4,4a,5,5a,6,11,12a-octahydrotetracene-2carboxamide (1d)

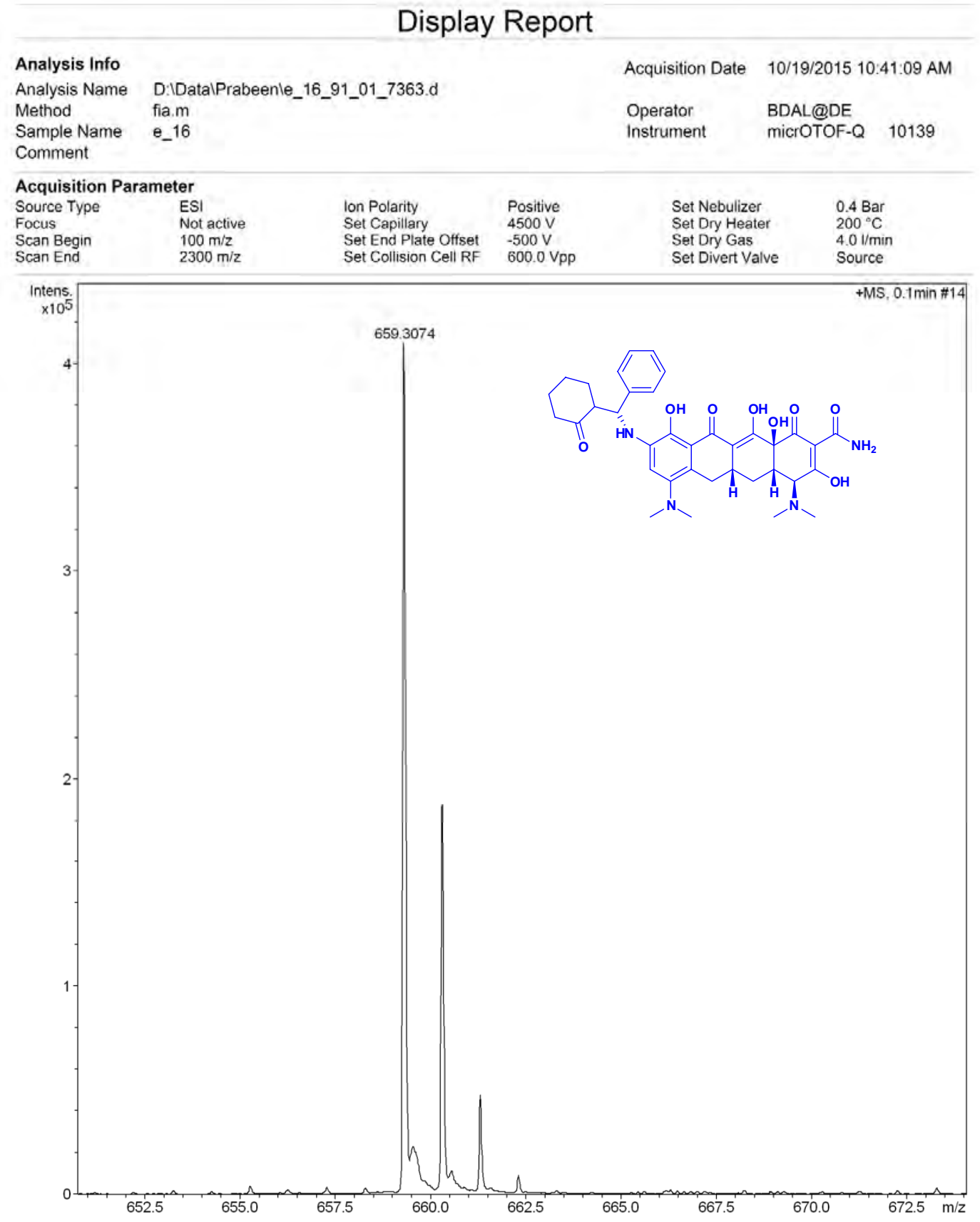


HRMS of (4S,4aS,5aR,12aS)-4,7-bis(dimethylamino)-3,10,12,12a-tetrahydroxy-9-((1S,2R)2-hydroxy-1-(4-nitrophenyl)-3-oxobutylamino)-1,11-dioxo-1,4,4a,5,5a,6,11,12aoctahydrotetracene-2-carboxamide (2a)

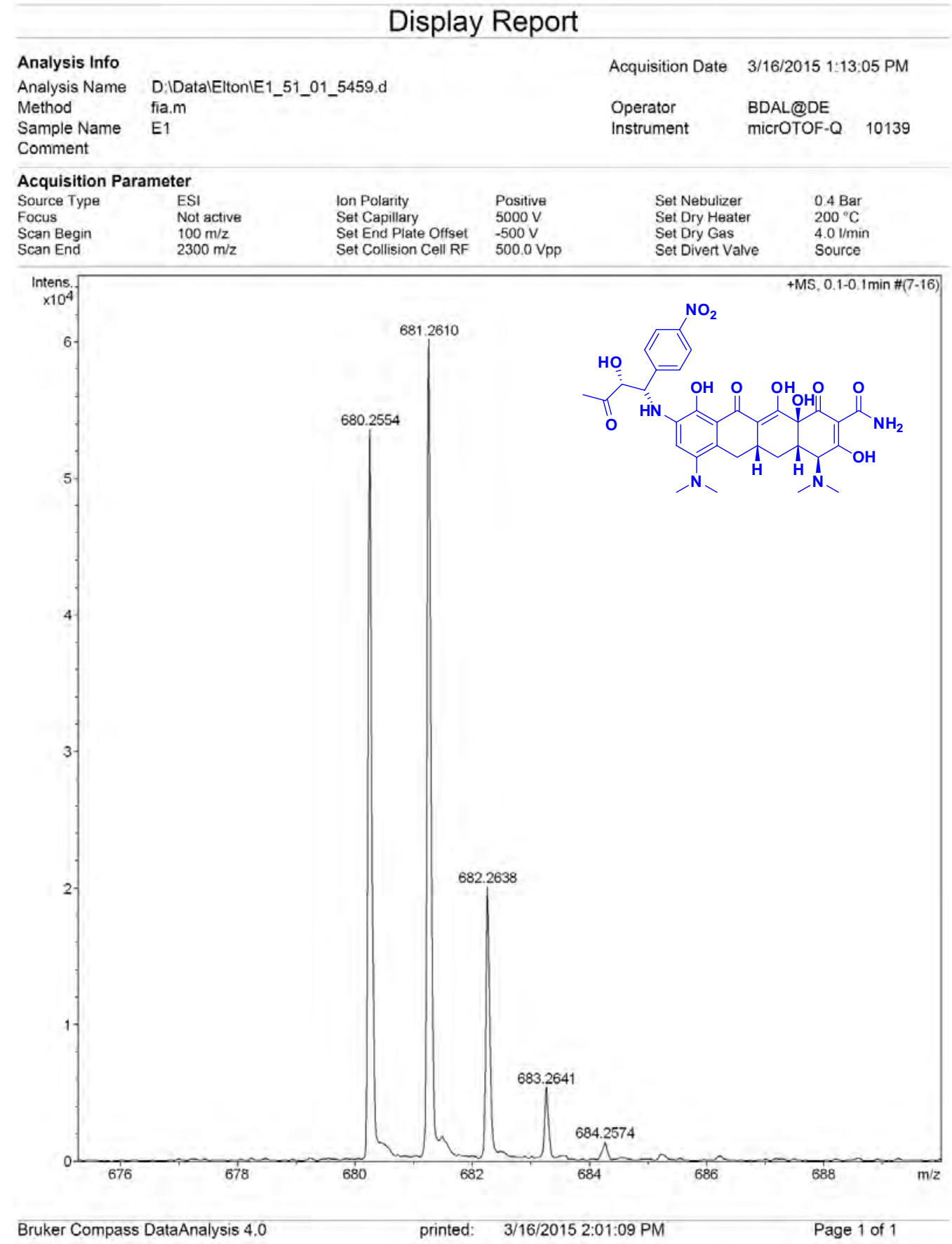


HRMS of (4S,4aS,5aR,12aS)-9-((1S,2R)-1-(4-butoxyphenyl)-2-hydroxy-3-oxobutylamino)4,7 bis(dimethylamino)-3,10,12,12a-tetrahydroxy-1,11-dioxo-1,4,4a,5,5a,6,11,12aoctahydrotetracene-2-carboxamide (2b)

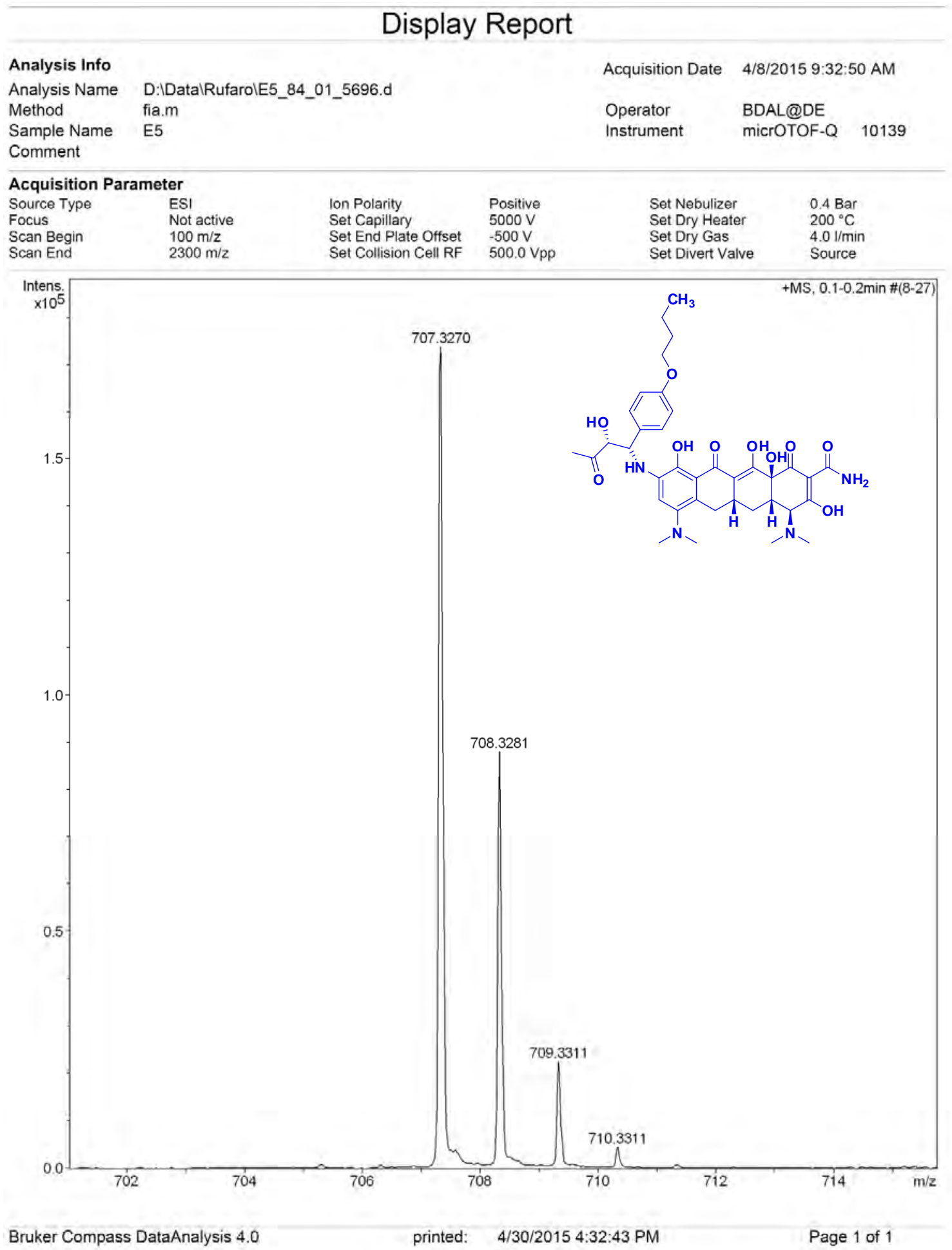


HRMS of (4S,4aS,5aR,12aS)-4,7-bis(dimethylamino)-3,10,12,12a-tetrahydroxy-9-((1S,2R)2-hydroxy-3-oxo-1-o-tolylbutylamino)-1,11-dioxo-1,4,4a,5,5a,6,11,12a-octahydrotetracene2-carboxamide (2c)

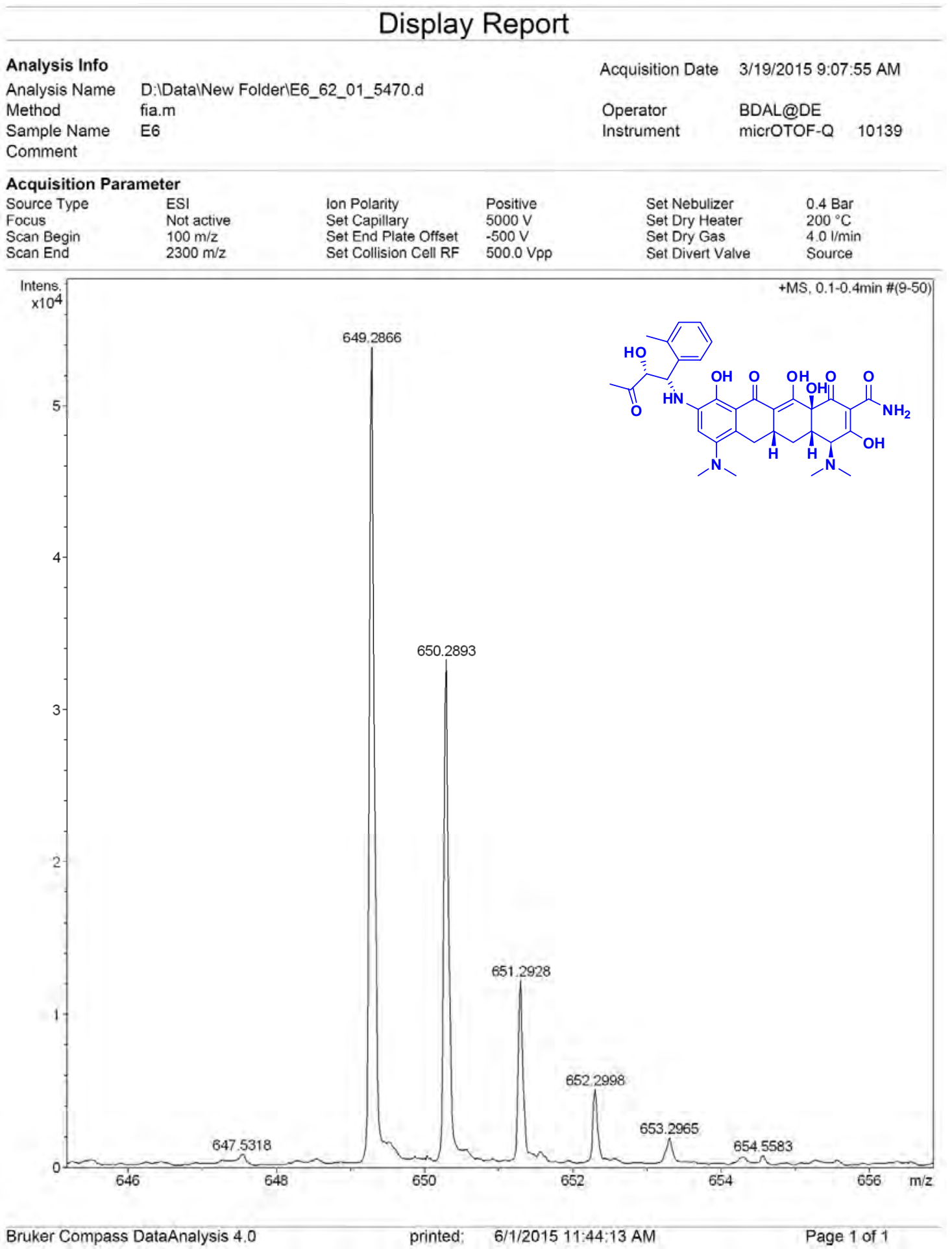


HRMS of (4S,4aS,5aR,12aS)-4,7-bis(dimethylamino)-9-((1S,2R)-1-(4-fluorophenyl)-2hydroxy-3-oxobutylamino)-3,10,12,12a-tetrahydroxy-1,11-dioxo-1,4,4a,5,5a,6,11,12aoctahydrotetracene-2-carboxamide (2d)

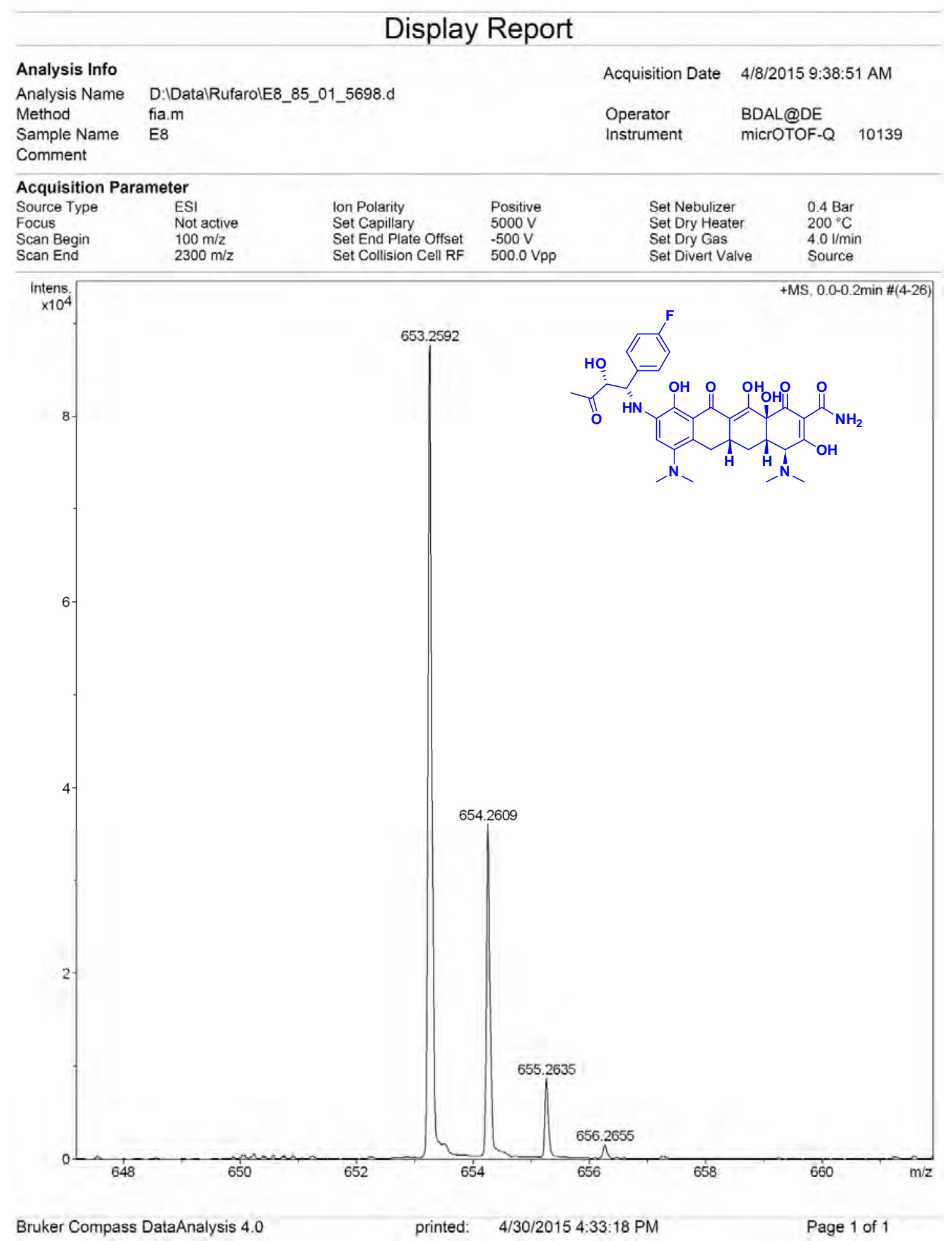


HRMS of (4S,4aS,5aR,12aS)-4,7-bis(dimethylamino)-3,10,12,12a-tetrahydroxy-9-((1S,2R)-2hydroxy-1-(naphthalen-2-yl)-3-oxobutylamino)-1,11-dioxo-1,4,4a,5,5a,6,11,12aoctahydrotetracene-2-carboxamide (2e)

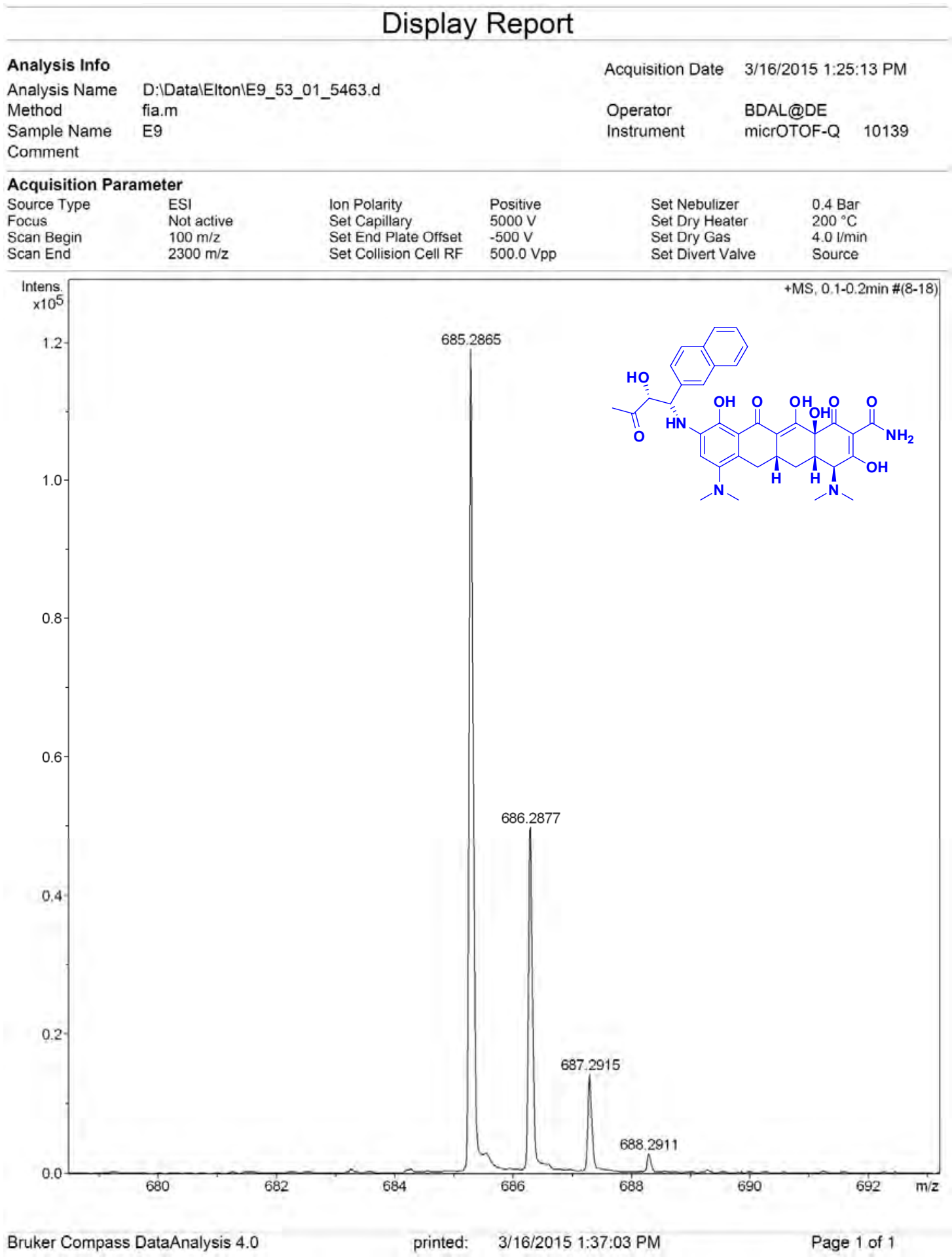


HRMS of (4S,4aS,5aR,12aS)-4,7-bis(dimethylamino)-3,10,12,12a-tetrahydroxy-9-((1R,2R)-

2-hydroxy-1-(5-nitrofuran-2-yl)-3-oxobutylamino)-1,11-dioxo-1,4,4a,5,5a,6,11,12

octahydrotetracene-2-carboxamide (2f)

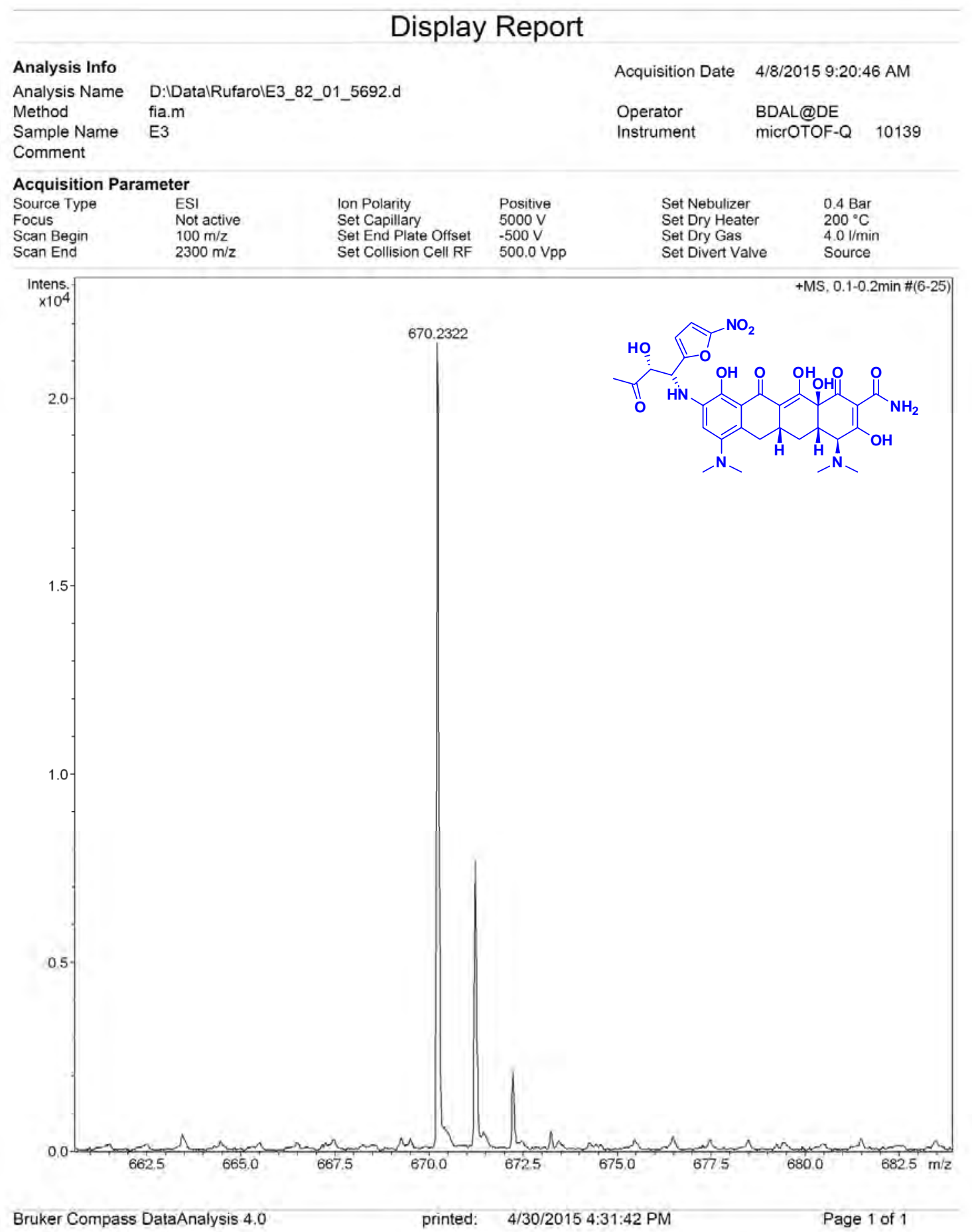


HRMS of (4S,4aS,5aR,12aS)-4,7-bis(dimethylamino)-9-((1R,2R)-1-(furan-2-yl)-2-hydroxy-

3-oxobutylamino)-3,10,12,12a-tetrahydroxy-1,11-dioxo-1,4,4a,5,5a,6,11,12aoctahydrotetracene-2-carboxamide (2g)

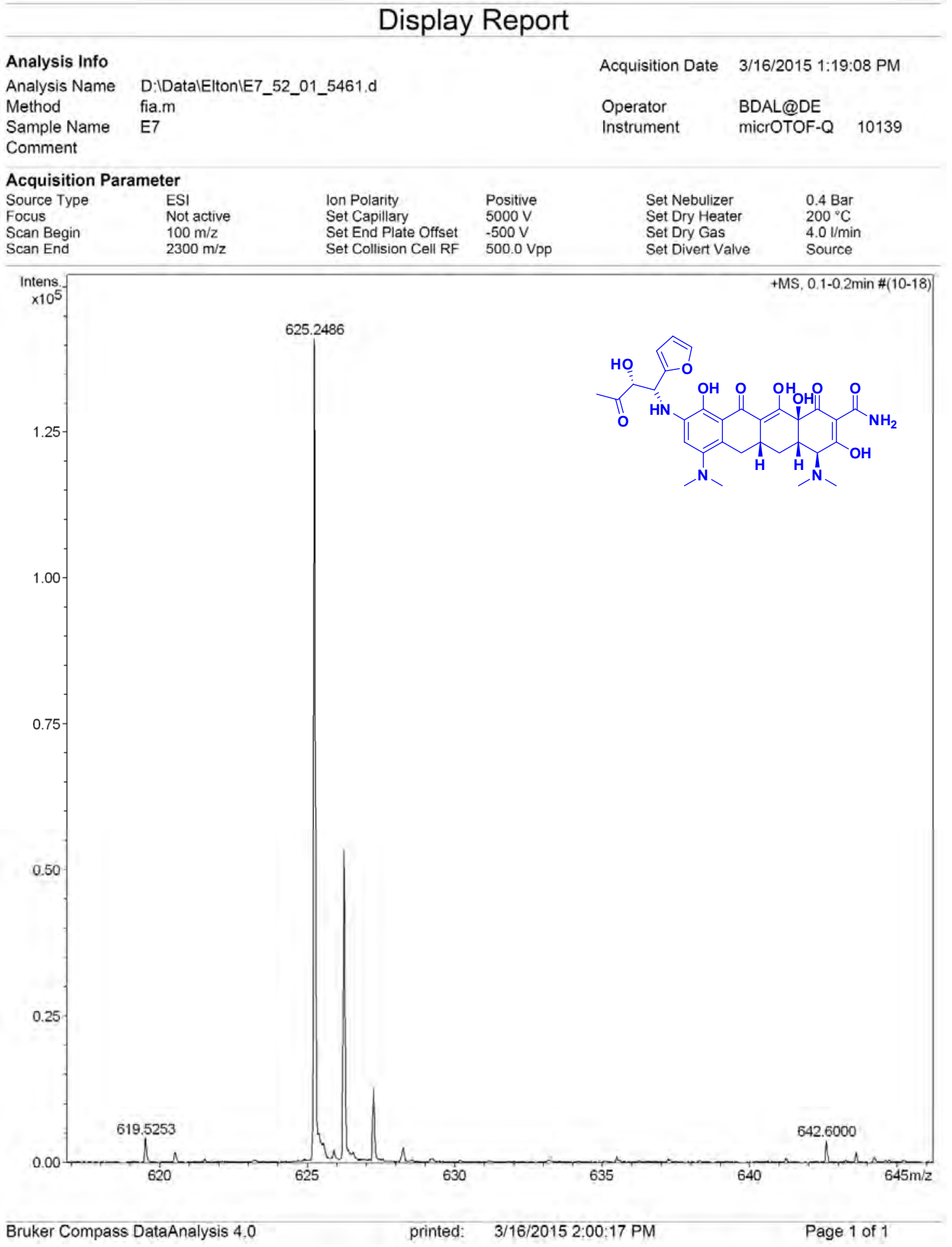


HRMS of (4S,4aS,5aR,12aS)-9-(1-(5-bromofuran-2-yl)-2-hydroxy-3-oxobutylamino)-4,7-bis (dimethylamino)-3,10,12,12a-tetrahydroxy-1,11-dioxo-1,4,4a,5,5a,6,11,12aoctahydrotetracene-2-carboxamide $(2 \mathrm{~h})$

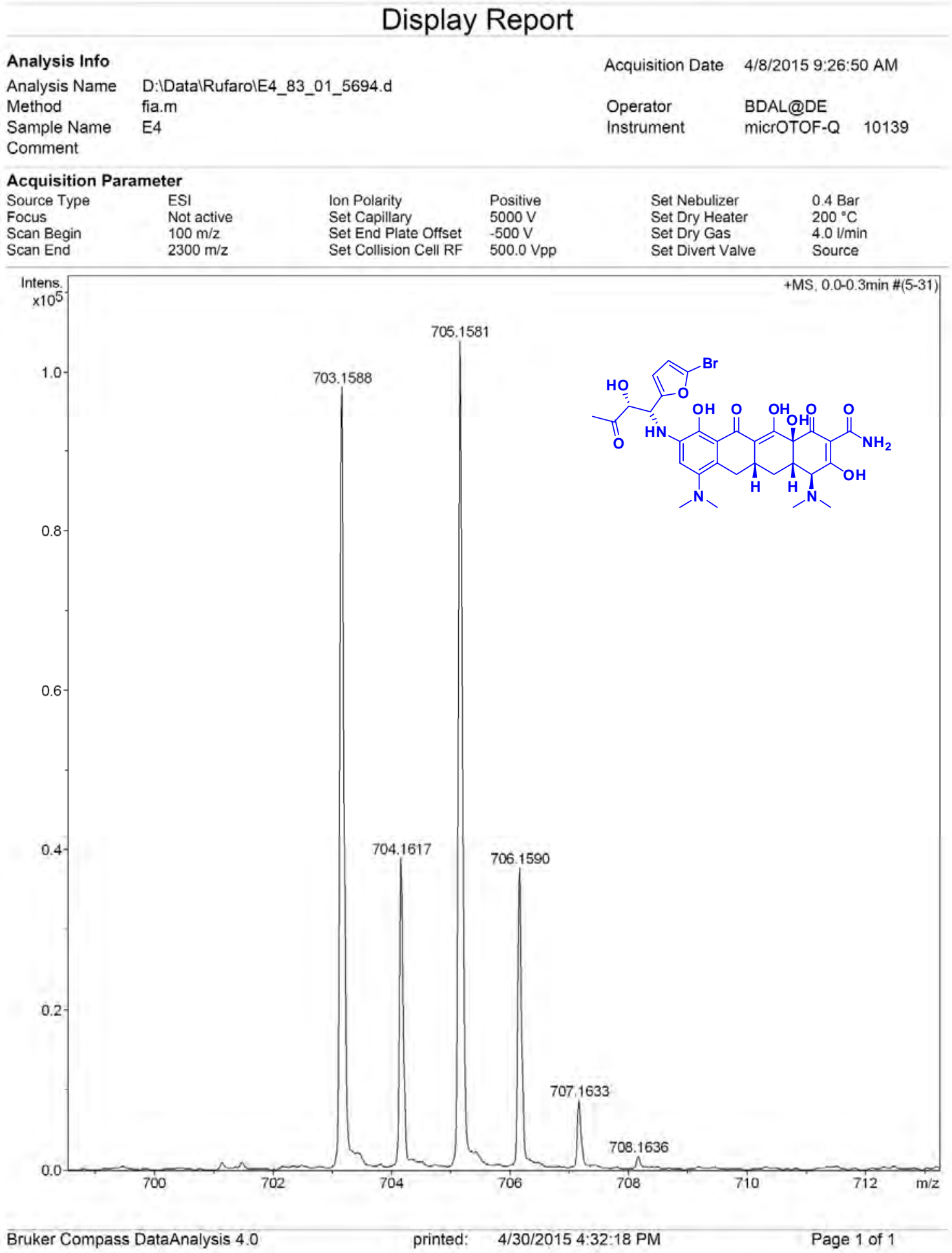




\section{Copies of LC-MS spectra for products}

LC-MS of (4S,4aS,5aR,12aS)-4,7-bis(dimethylamino)-3,10,12,12a-tetrahydroxy-9-(2hydroxy-3-oxo-1-phenylbutylamino)-1,11-dioxo-1,4,4a,5,5a,6,11,12a-octahydrotetracene-2carboxamide (1a)

\section{==== Shimadzu LabSolutions Multi-Chromatogram ====}

mAU
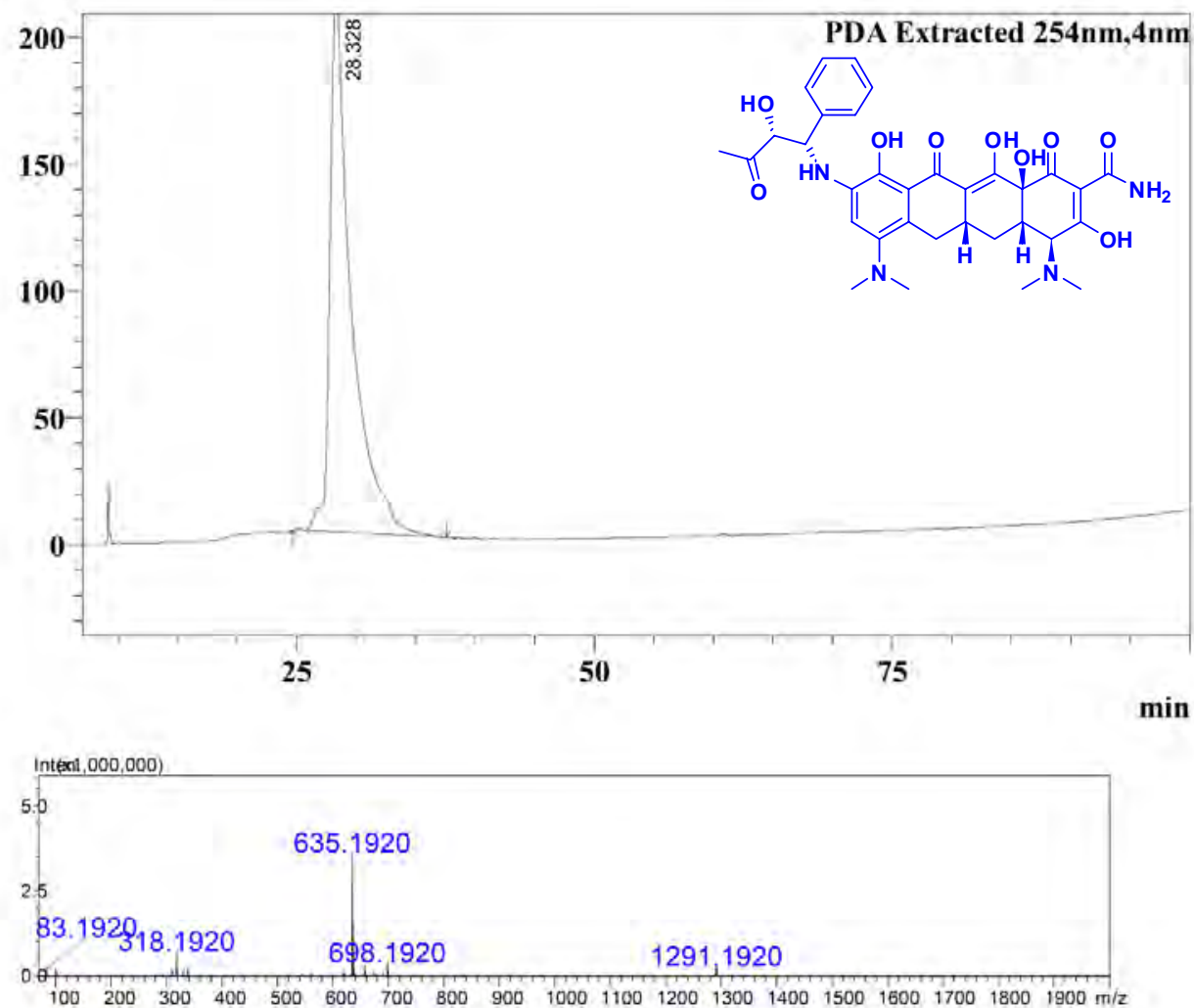
LC-MS of (4S,4aS,5aR,12aS)-4,7-bis(dimethylamino)-3,10,12,12a-tetrahydroxy-9-(2methyl-3-oxo-1-phenylbutylamino)-1,11-dioxo-1,4,4a,5,5a,6,11,12a-octahydrotetracene-2carboxamide (1b)

14/10/2015 16:06:09 Page $1 / 5$

==== Shimadzu LabSolutions Multi-Chromatogram ==== $\mathrm{mAU}$
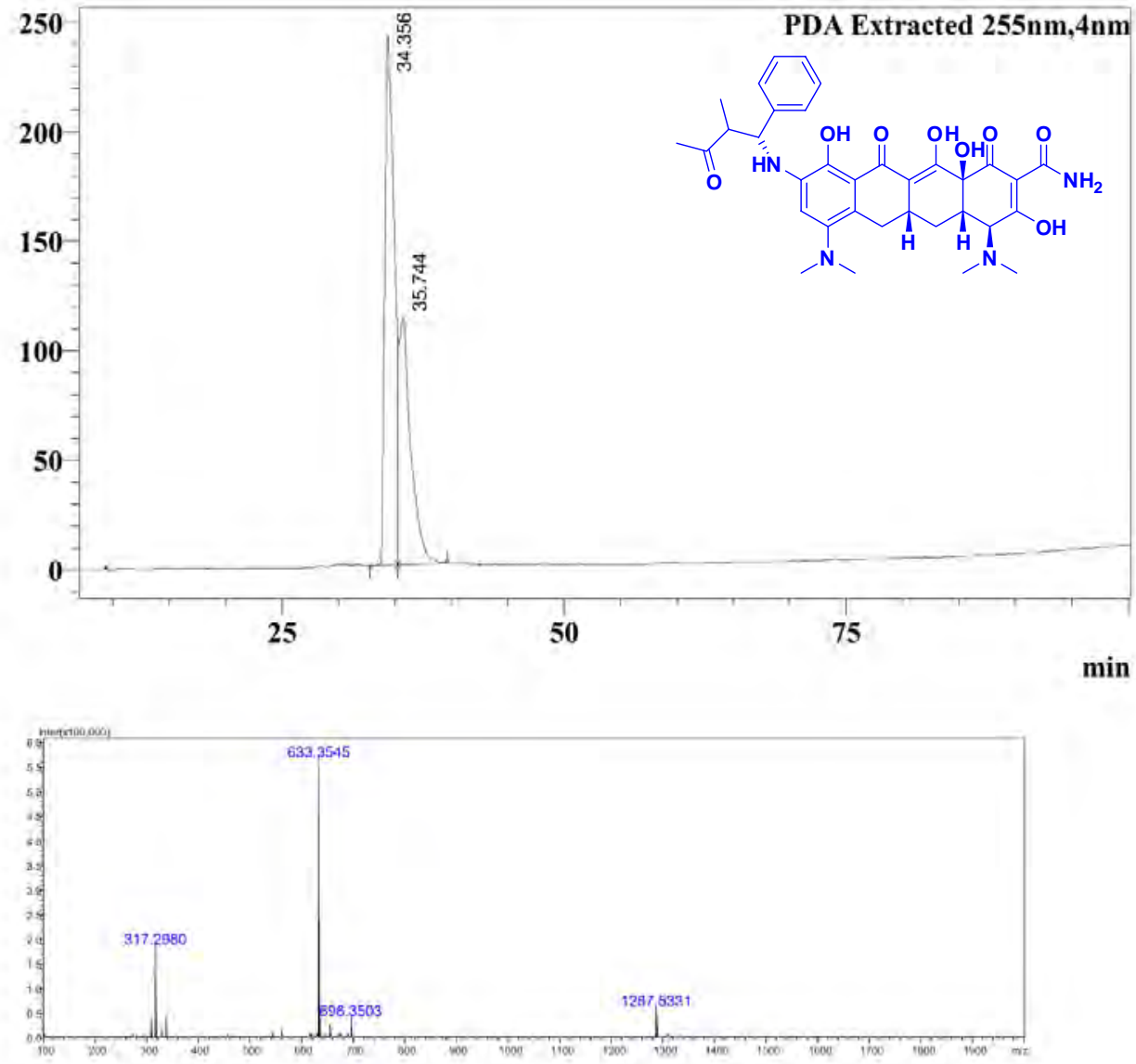
LC-MS of (4S,4aS,5aR,12aS)-4,7-bis(dimethylamino)-3,10,12,12a-tetrahydroxy-1,11-dioxo9-(3-oxo-1-phenylbutylamino)-1,4,4a,5,5a,6,11,12a-octahydrotetracene-2-carboxamide (1c)

17/10/2015 18:11:31 Page 1/5
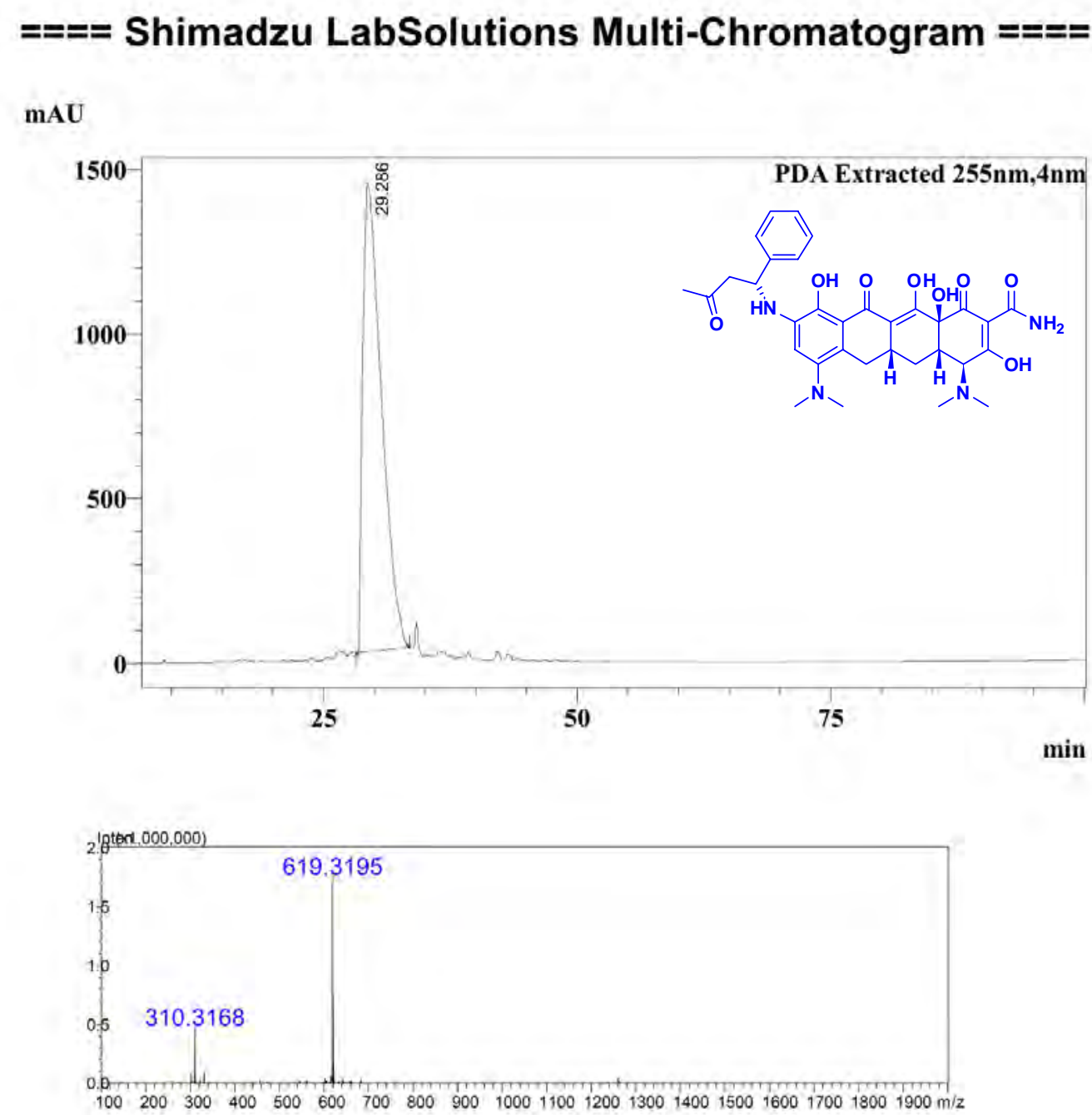
LC-MS of (4S,4aS,5aR,12aS)-4,7-bis(dimethylamino)-3,10,12,12a-tetrahydroxy-1,11-dioxo9-((1R)-(2-oxocyclohexyl)(phenyl)methylamino)-1,4,4a,5,5a,6,11,12a-octahydrotetracene-2carboxamide (1d)

\section{=ニ== Shimadzu LabSolutions Multi-Chromatogram ==== mAU}
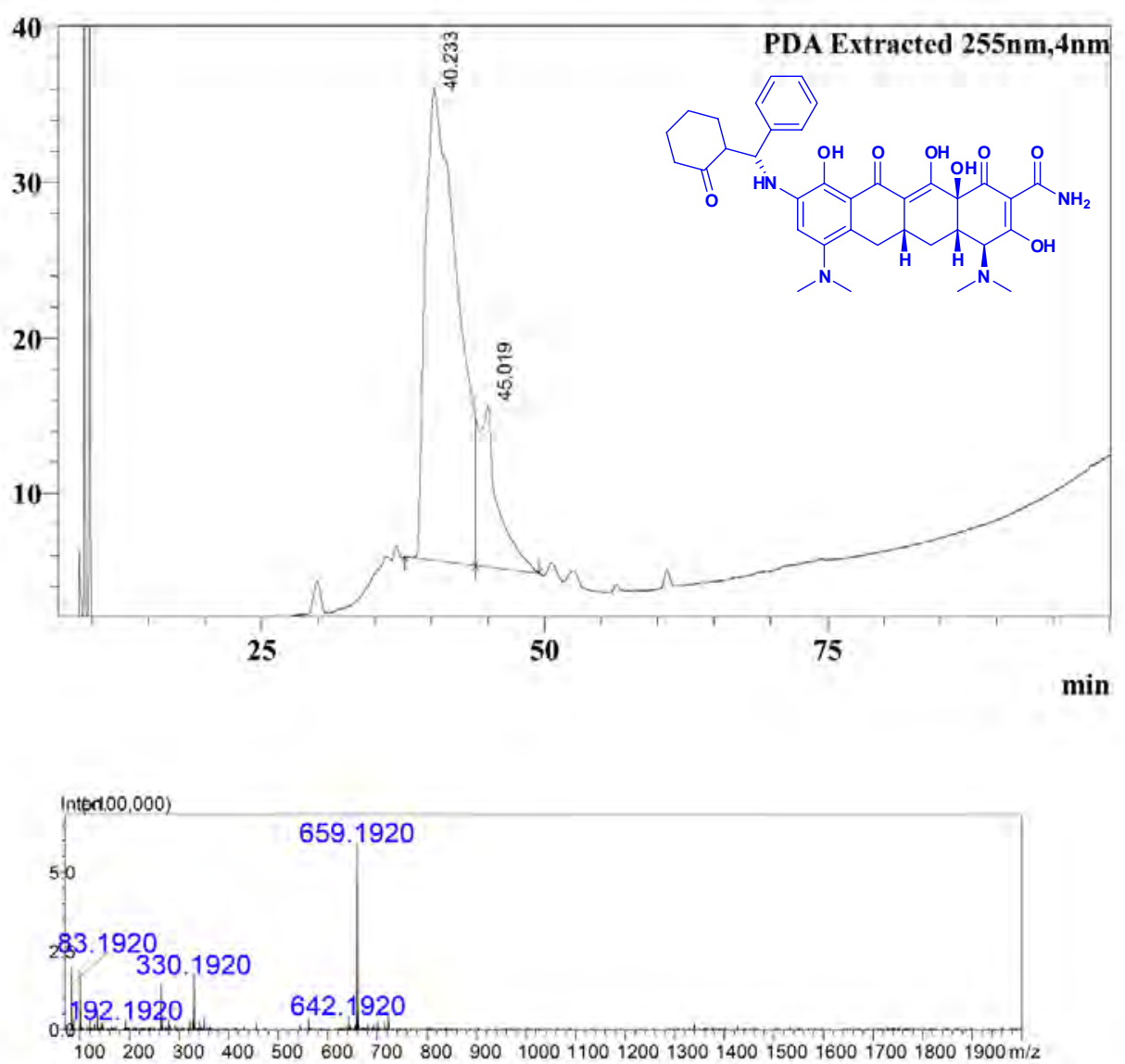
LC-MS of (4S,4aS,5aR,12aS)-4,7-bis(dimethylamino)-3,10,12,12a-tetrahydroxy-9-((1S,2R)2-hydroxy-1-(4-nitrophenyl)-3-oxobutylamino)-1,11-dioxo-1,4,4a,5,5a,6,11,12aoctahydrotetracene-2-carboxamide (2a)

\section{==== Shimadzu LabSolutions Multi-Chromatogram ====} mAU
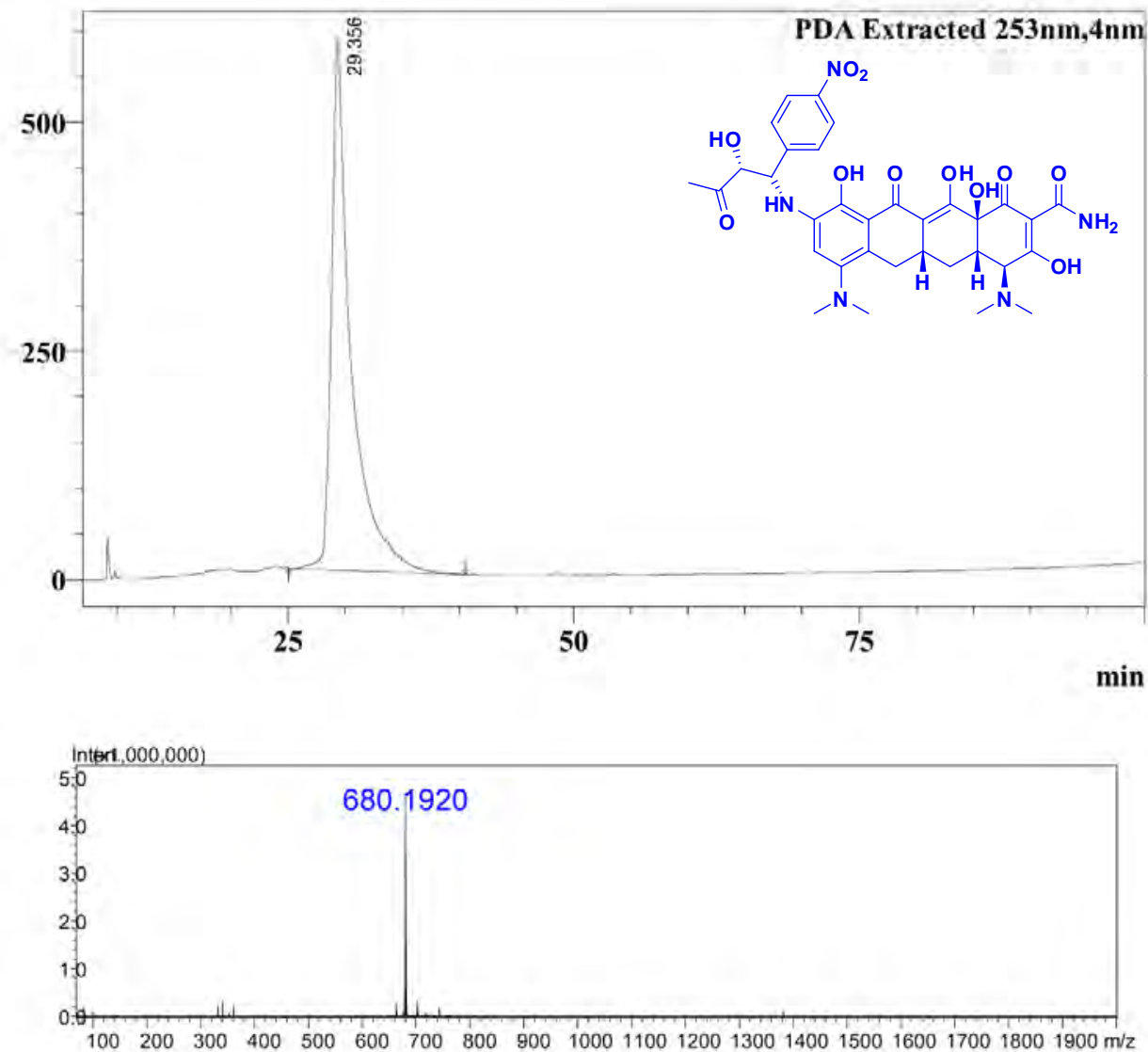
LC-MS of (4S,4aS,5aR,12aS)-9-((1S,2R)-1-(4-butoxyphenyl)-2-hydroxy-3-oxobutylamino)4,7 bis(dimethylamino)-3,10,12,12a-tetrahydroxy-1,11-dioxo-1,4,4a,5,5a,6,11,12aoctahydrotetracene-2-carboxamide (2b)

14/10/2015 15:29:14 Page 1
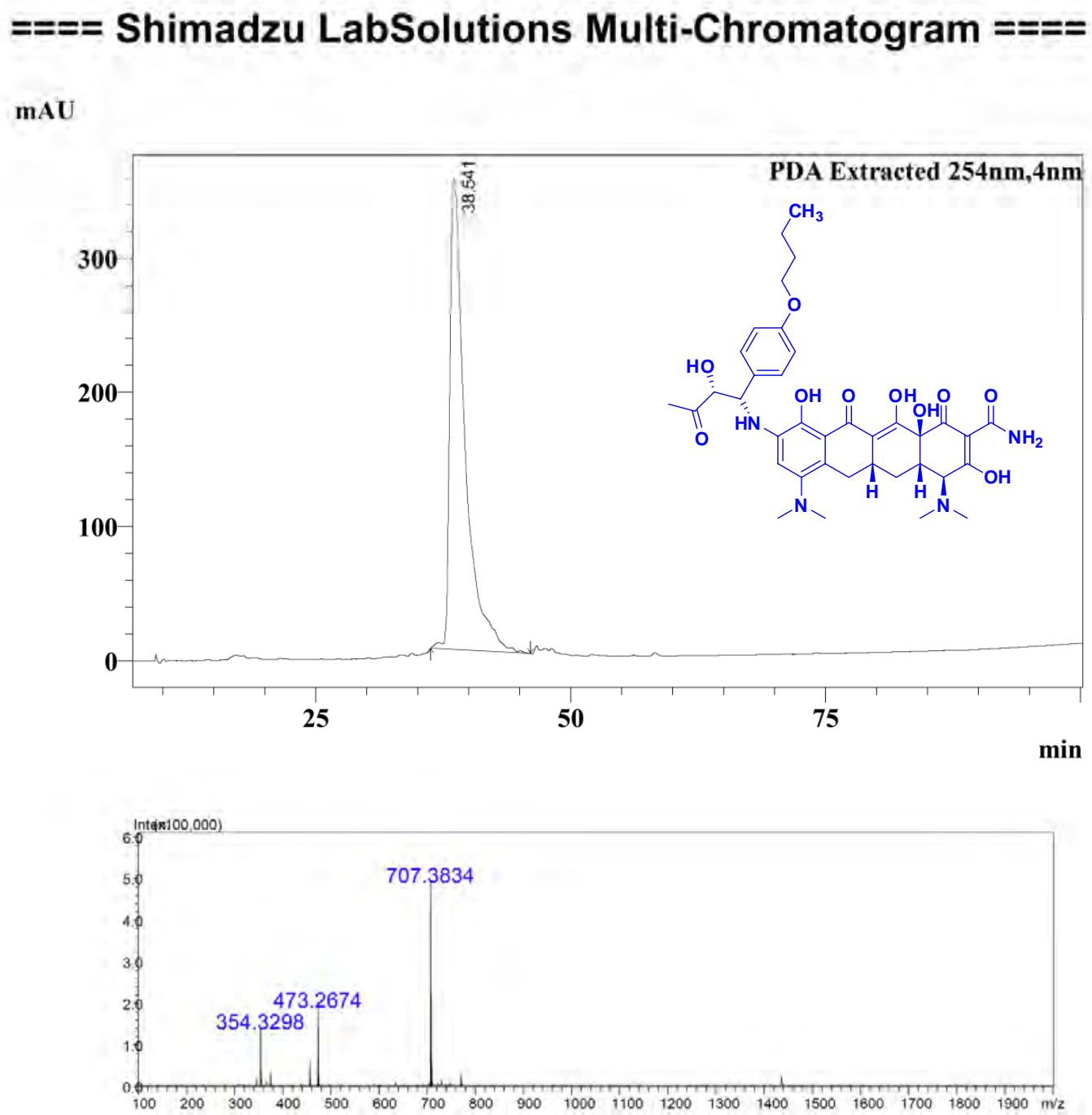
LC-MS of (4S,4aS,5aR,12aS)-4,7-bis(dimethylamino)-3,10,12,12a-tetrahydroxy-9-((1S,2R)2-hydroxy-3-oxo-1-o-tolylbutylamino)-1,11-dioxo-1,4,4a,5,5a,6,11,12a-octahydrotetracene2-carboxamide (2c)
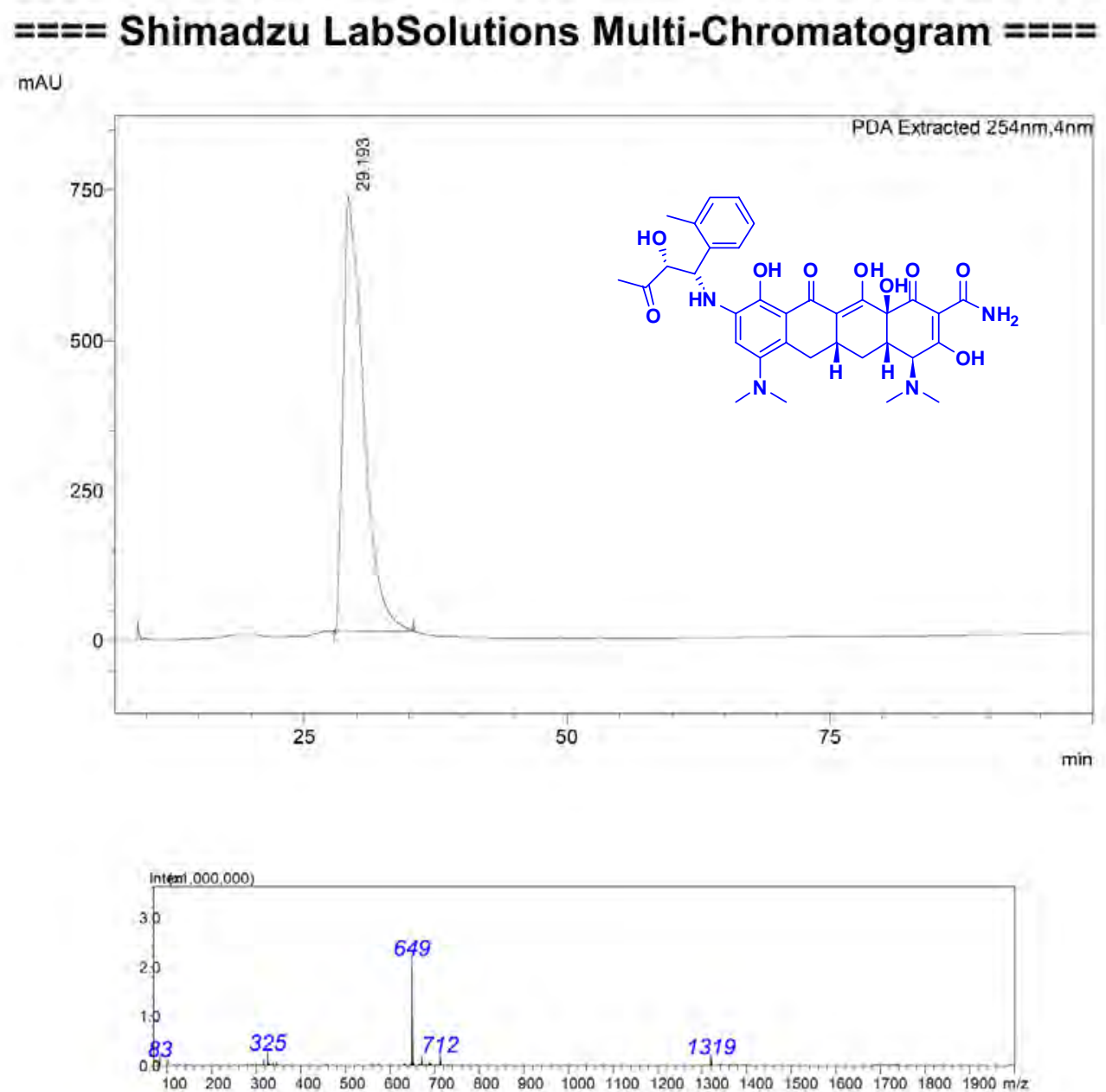
LC-MS SPECTRUM of (4S,4aS,5aR,12aS)-4,7-bis(dimethylamino)-9-((1S,2R)-1-(4fluorophenyl)-2-hydroxy-3-oxobutylamino)-3,10,12,12a-tetrahydroxy-1,11-dioxo1,4,4a,5,5a,6,11,12a-octahydrotetracene-2-carboxamide (2d)

==== Shimadzu LabSolutions Multi-Chromatogram ====

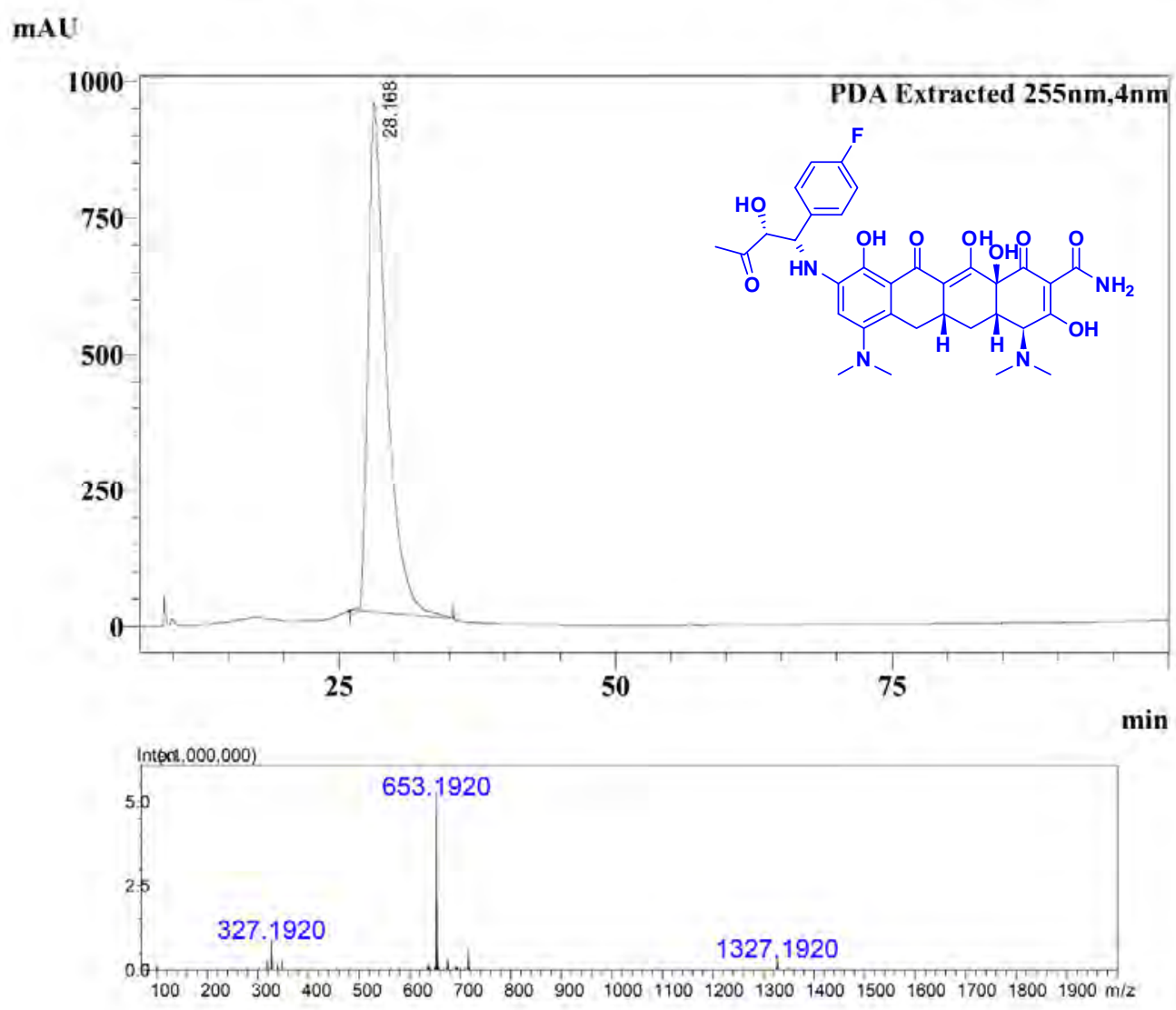


LC-MS of (4S,4aS,5aR,12aS)-4,7-bis(dimethylamino)-3,10,12,12a-tetrahydroxy-9-((1S,2R)2-hydroxy-1-(naphthalen-2-yl)-3-oxobutylamino)-1,11-dioxo-1,4,4a,5,5a,6,11,12aoctahydrotetracene-2-carboxamide (2e)

==== Shimadzu LabSolutions Multi-Chromatogram ==== mAU
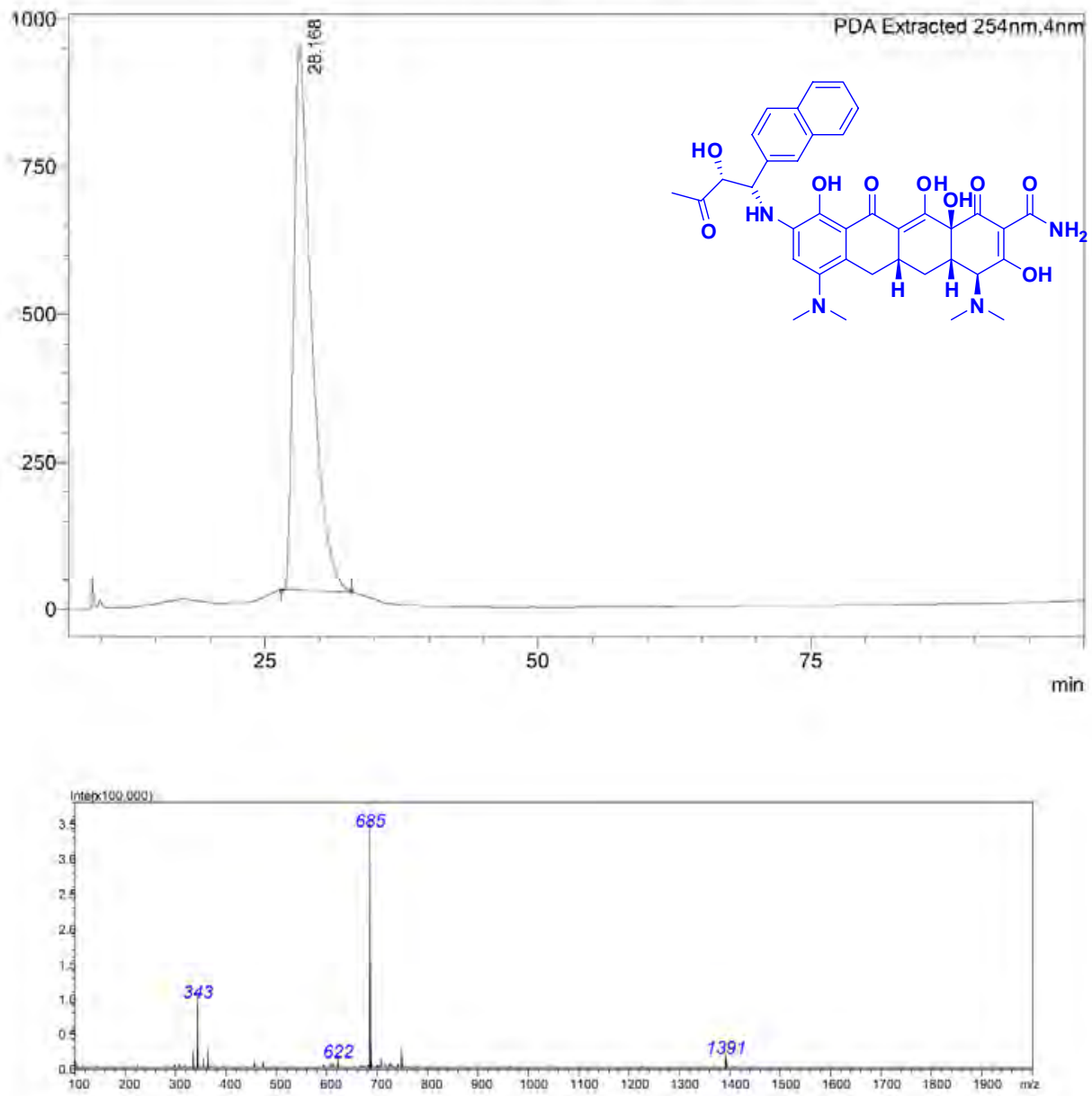
LC-MS of (4S,4aS,5aR,12aS)-4,7-bis(dimethylamino)-3,10,12,12a-tetrahydroxy-9-((1R,2R)2-hydroxy-1-(5-nitrofuran-2-yl)-3-oxobutylamino)-1,11-dioxo-1,4,4a,5,5a,6,11,12 octahydrotetracene-2-carboxamide (2f)

\section{=ニ== Shimadzu LabSolutions Multi-Chromatogram ==== mAU}
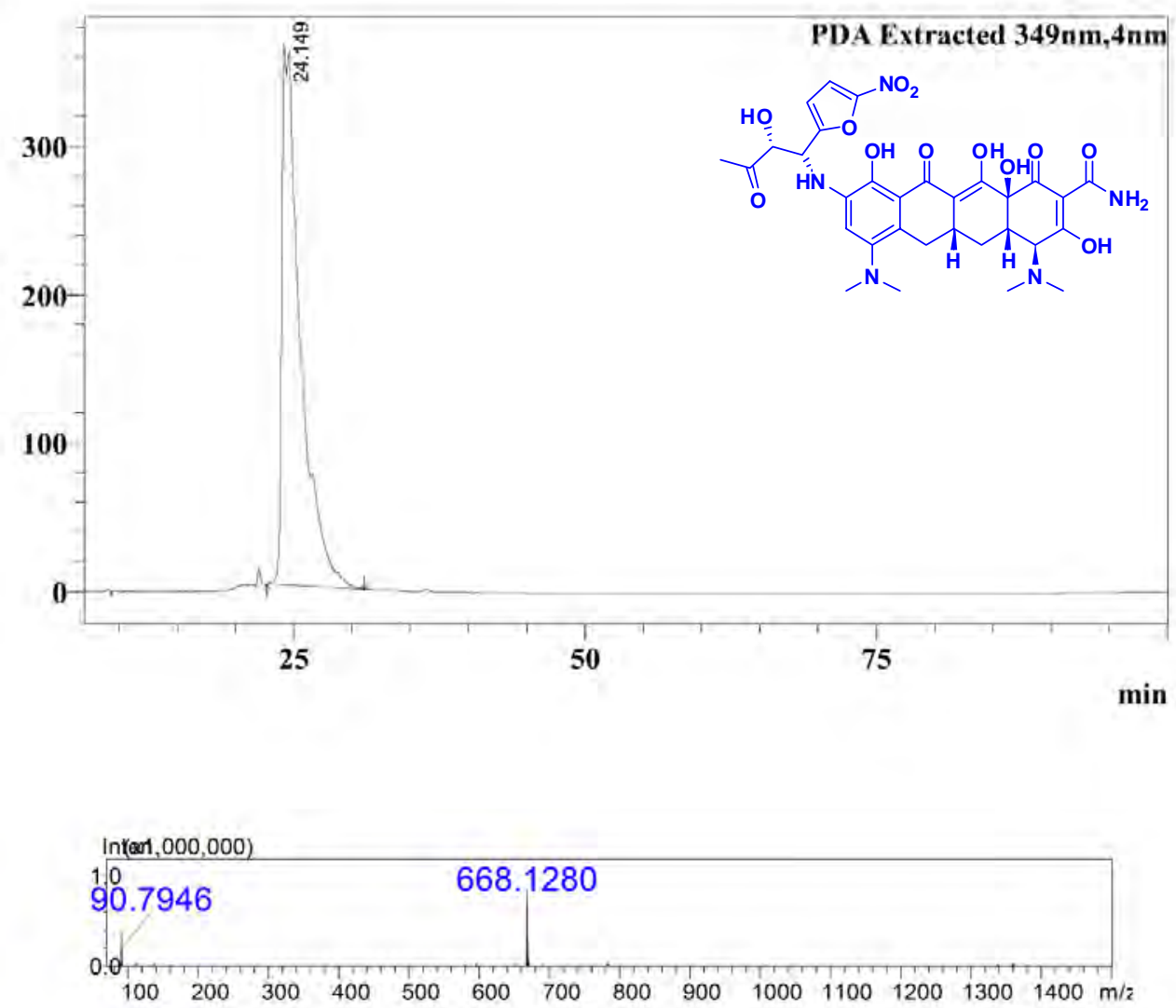
LC-MS of (4S,4aS,5aR,12aS)-4,7-bis(dimethylamino)-9-((1R,2R)-1-(furan-2-yl)-2-hydroxy3-oxobutylamino)-3,10,12,12a-tetrahydroxy-1,11-dioxo-1,4,4a,5,5a,6,11,12aoctahydrotetracene-2-carboxamide (2g)
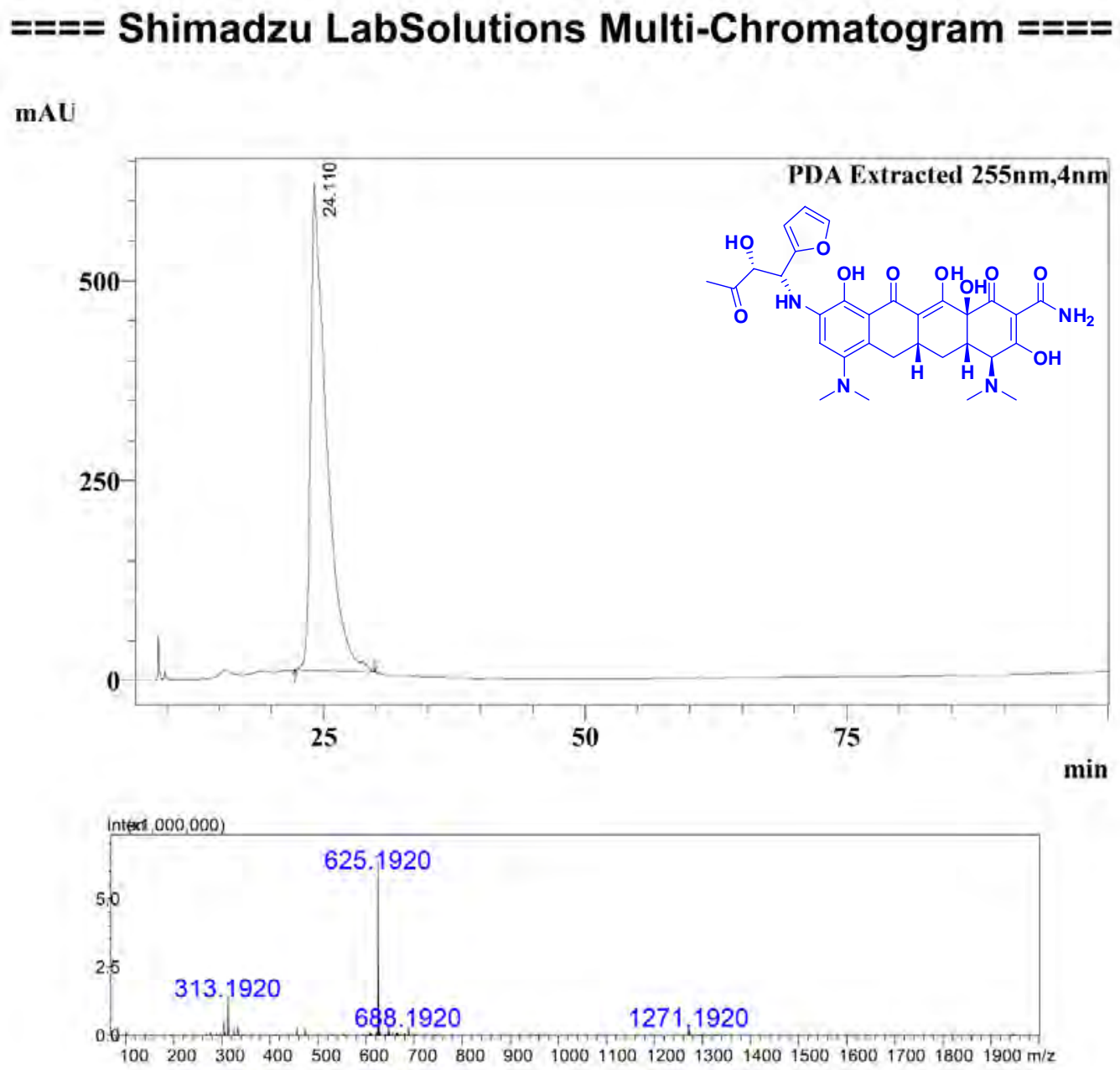
LC-MS of (4S,4aS,5aR,12aS)-9-(1-(5-bromofuran-2-yl)-2-hydroxy-3-oxobutylamino)-4,7bis (dimethylamino)-3,10,12,12a-tetrahydroxy-1,11-dioxo-1,4,4a,5,5a,6,11,12aoctahydrotetracene-2-carboxamide (2h)

14/10/2015 15:15:01 Page 1

==== Shimadzu LabSolutions Multi-Chromatogram ==== mAU
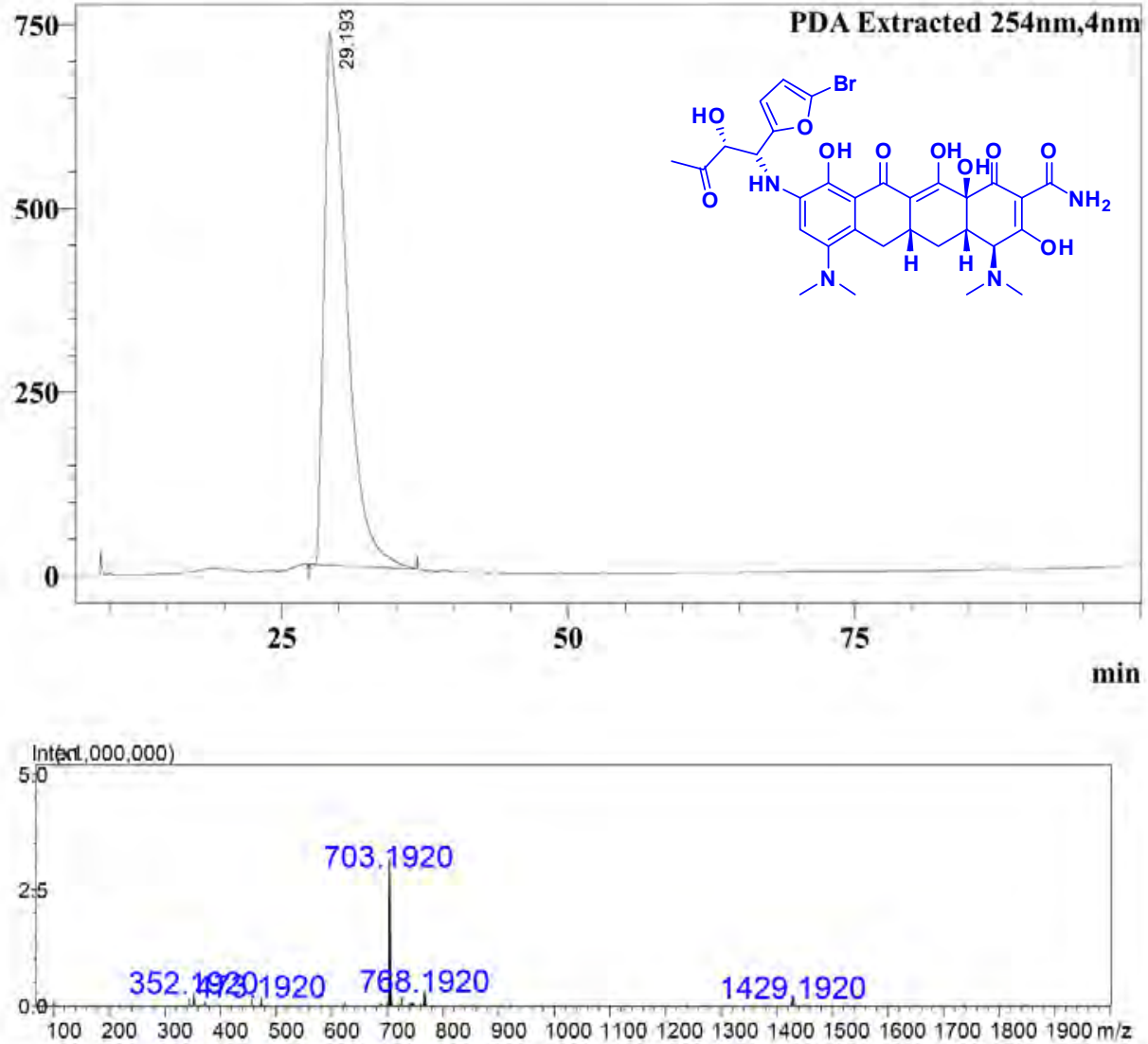


\section{Copies of NMR spectra for products}

${ }^{1} \mathrm{H}$ NMR of (4S,4aS,5aR,12aS)-4,7-bis(dimethylamino)-3,10,12,12a-tetrahydroxy-9-(2-hydroxy-3-oxo-1-phenylbutylamino)1,11-dioxo-1,4,4a,5,5a,6,11,12a-octahydrotetracene-2-carboxamide (1a)

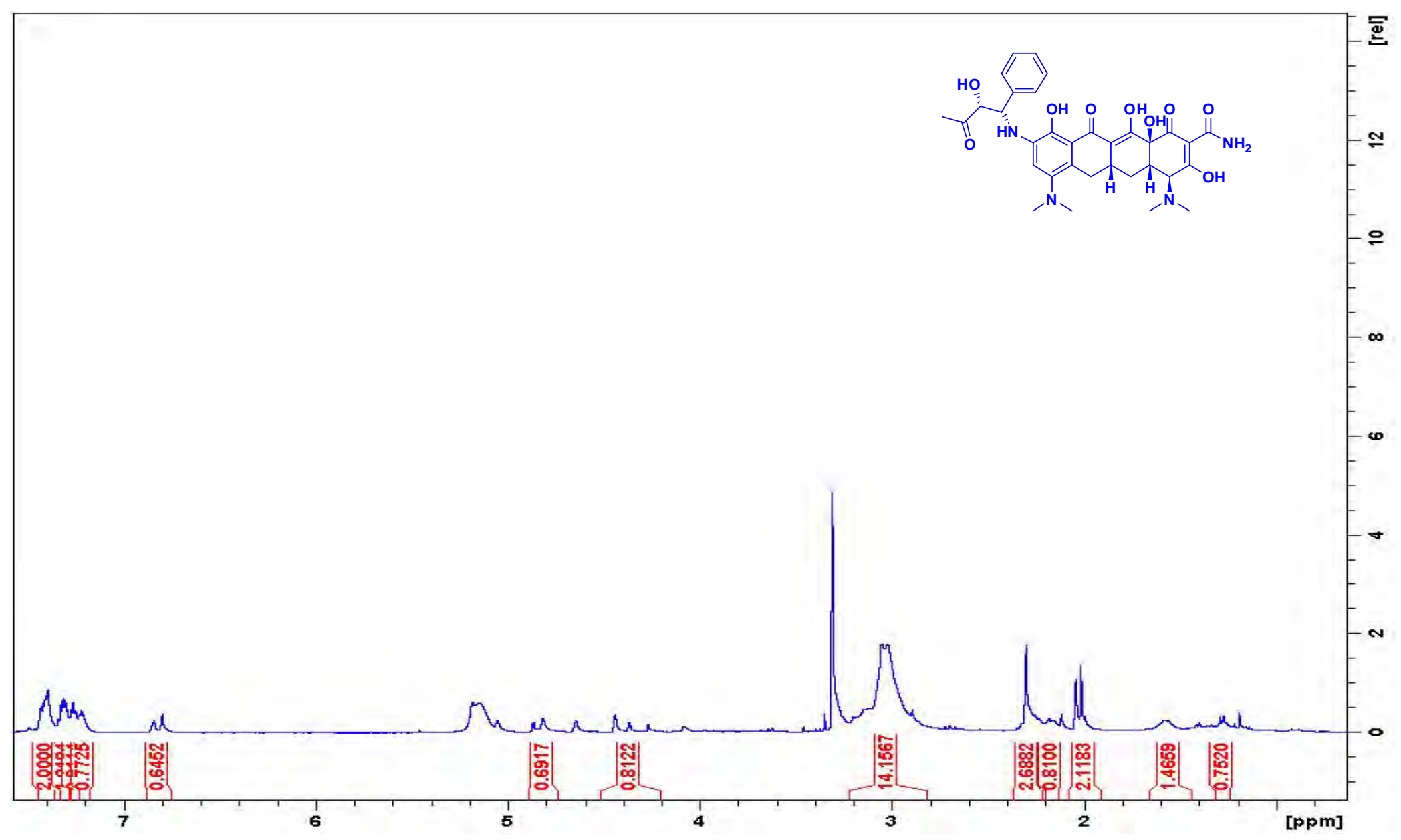


${ }^{1} \mathrm{H}$ NMR of (4S,4aS,5aR,12aS)-4,7-bis(dimethylamino)-3,10,12,12a-tetrahydroxy-9-(2-methyl-3-oxo-1-phenylbutylamino)-1,11dioxo-1,4,4a,5,5a,6,11,12a-octahydrotetracene-2-carboxamide (1b)

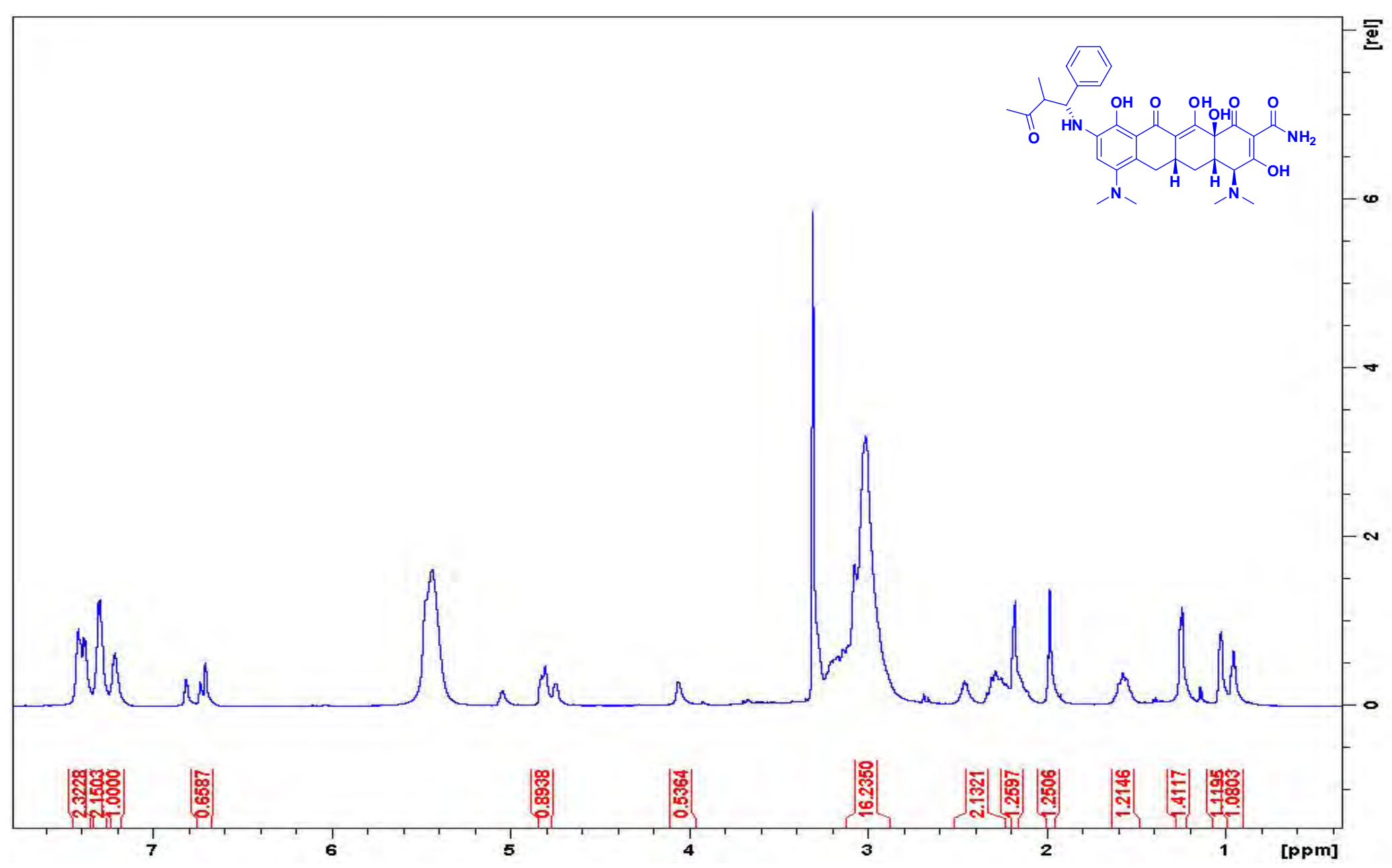


${ }^{1}$ H NMR of (4S,4aS,5aR,12aS)-4,7-bis(dimethylamino)-3,10,12,12a-tetrahydroxy-1,11-dioxo-9-(3-oxo-1-phenylbutylamino)1,4,4a,5,5a,6,11,12a-octahydrotetracene-2-carboxamide (1c)

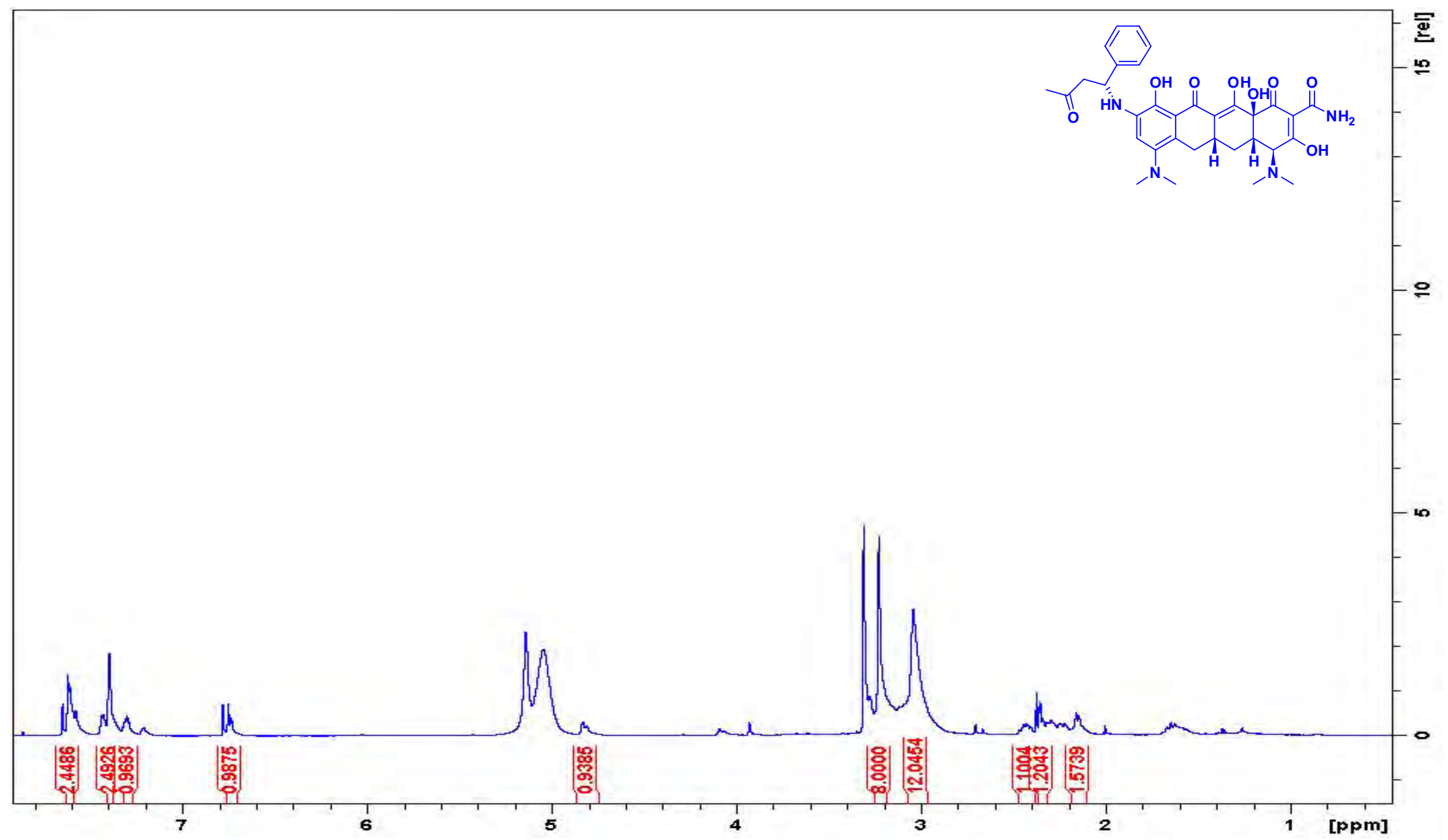


${ }^{1} \mathrm{H}$ NMR of (4S,4aS,5aR,12aS)-4,7-bis(dimethylamino)-3,10,12,12a-tetrahydroxy-1,11-dioxo-9-((1R)-(2oxocyclohexyl)(phenyl)methylamino)-1,4,4a,5,5a,6,11,12a-octahydrotetracene-2-carboxamide (1d)

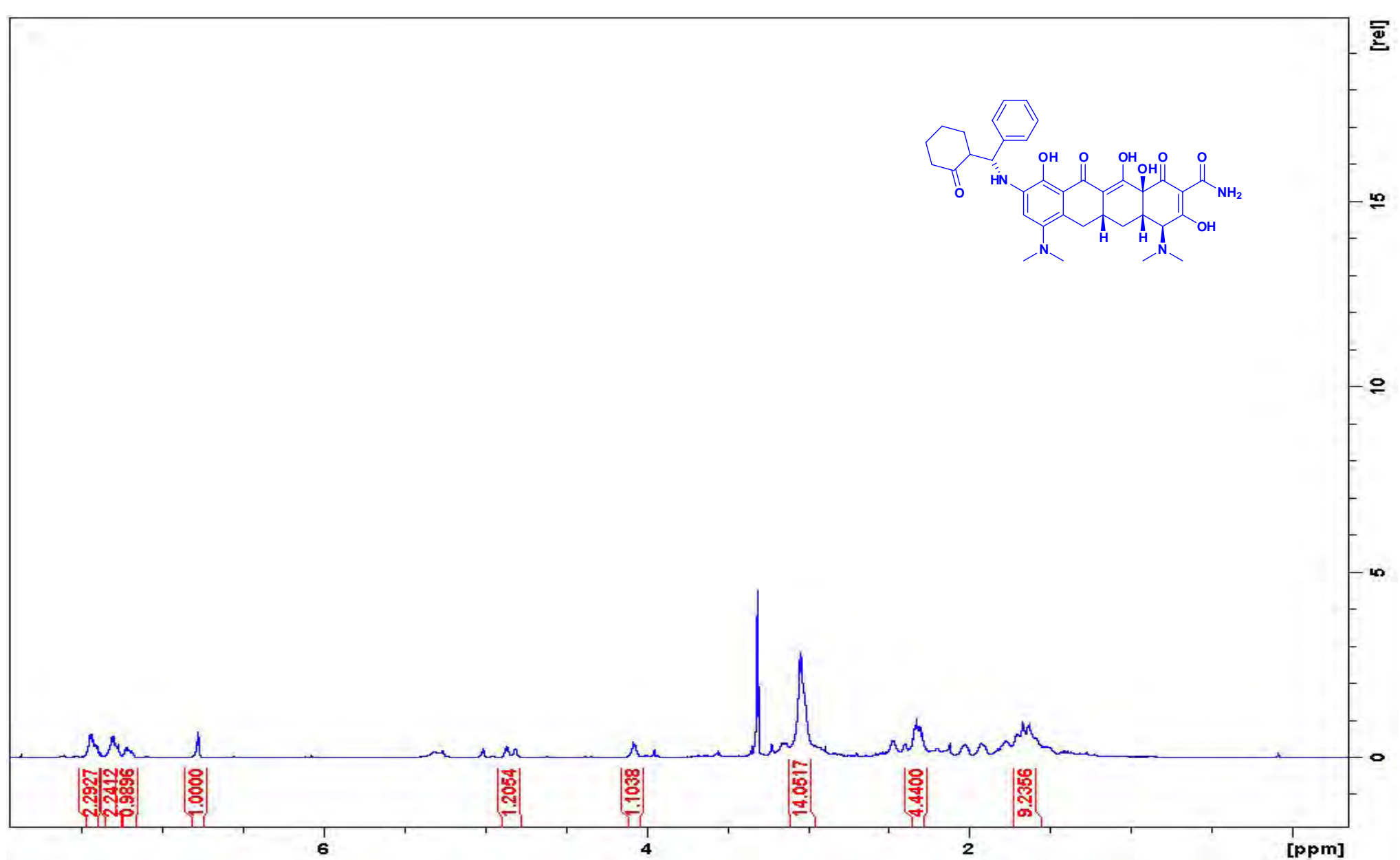


${ }^{1}$ H NMR of (4S,4aS,5aR,12aS)-4,7-bis(dimethylamino)-3,10,12,12a-tetrahydroxy-9-((1S,2R)-2-hydroxy-1-(4-nitrophenyl)-3oxobutylamino)-1,11-dioxo-1,4,4a,5,5a,6,11,12a-octahydrotetracene-2-carboxamide (2a)

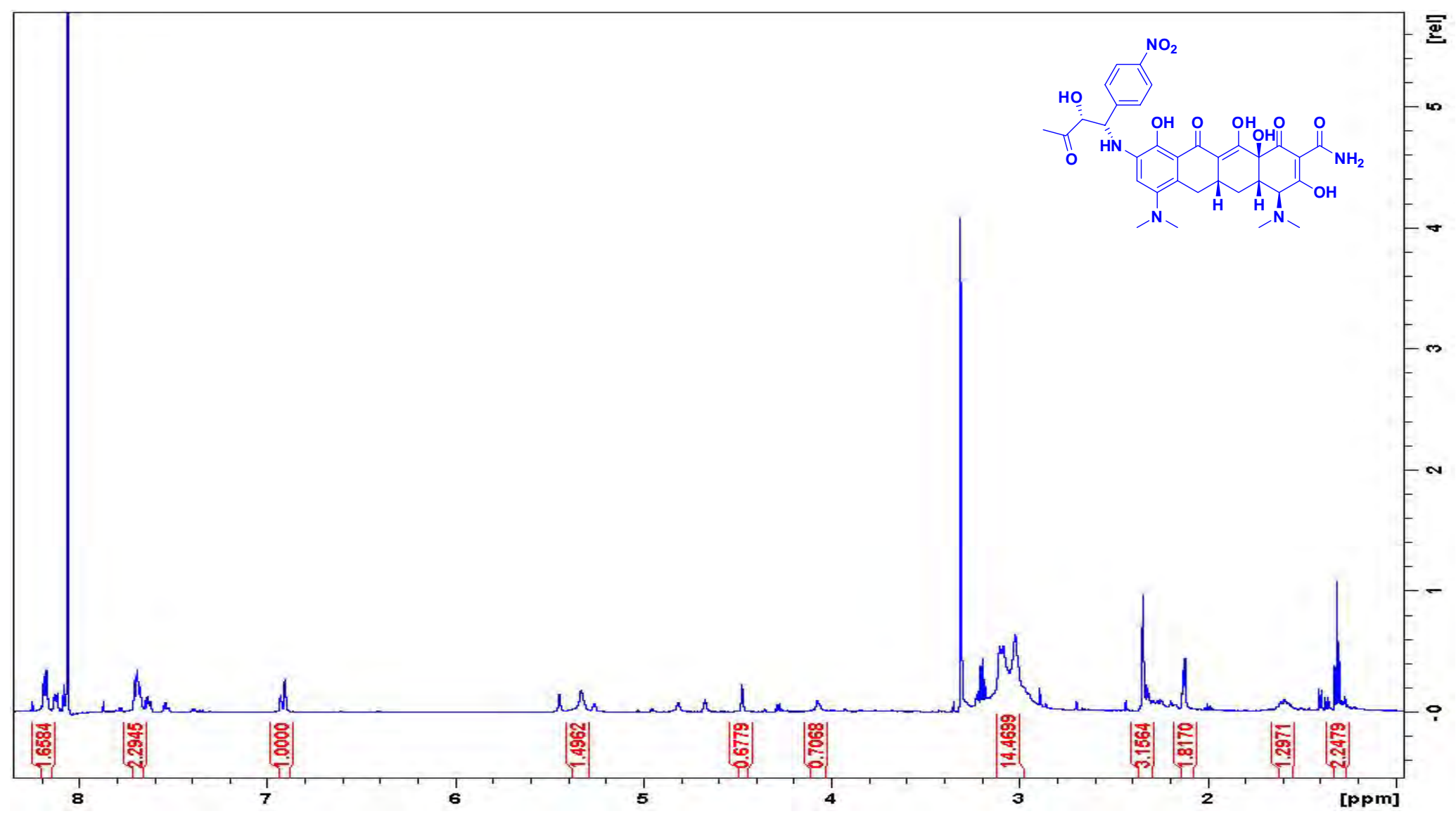


${ }^{1}$ H NMR of (4S,4aS,5aR,12aS)-9-((1S,2R)-1-(4-butoxyphenyl)-2-hydroxy-3-oxobutylamino)-4,7 bis(dimethylamino)3,10,12,12a-tetrahydroxy-1,11-dioxo-1,4,4a,5,5a,6,11,12a-octahydrotetracene-2-carboxamide (2b)

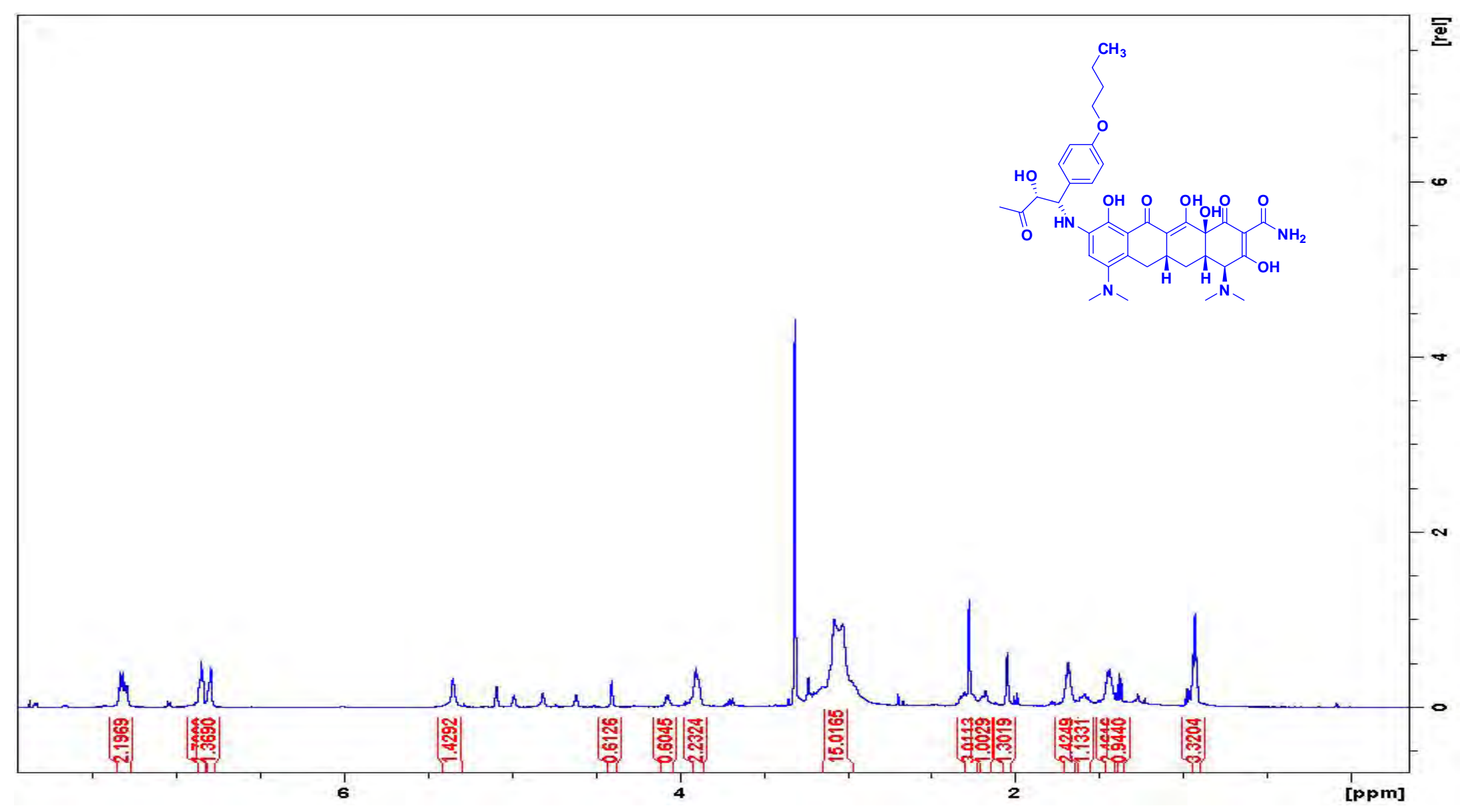


${ }^{1} \mathrm{H}$ NMR of (4S,4aS,5aR,12aS)-4,7-bis(dimethylamino)-3,10,12,12a-tetrahydroxy-9-((1S,2R)-2-hydroxy-3-oxo-1-otolylbutylamino)-1,11-dioxo-1,4,4a,5,5a,6,11,12a-octahydrotetracene-2-carboxamide (2c)

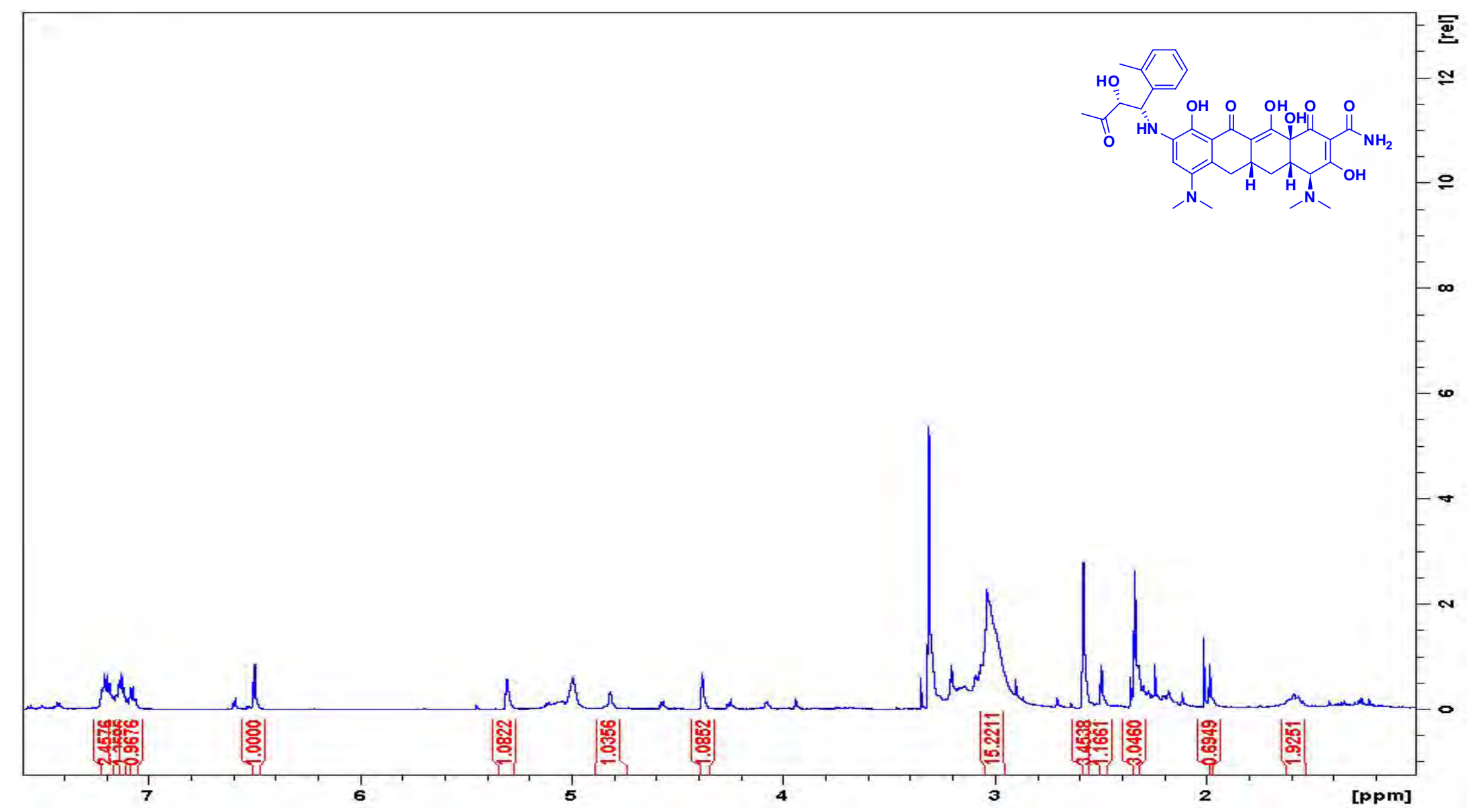


${ }^{1} \mathrm{H}$ NMR of (4S,4aS,5aR,12aS)-4,7-bis(dimethylamino)-9-((1S,2R)-1-(4-fluorophenyl)-2-hydroxy-3-oxobutylamino)3,10,12,12a-tetrahydroxy-1,11-dioxo-1,4,4a,5,5a,6,11,12a-octahydrotetracene-2-carboxamide (2d)

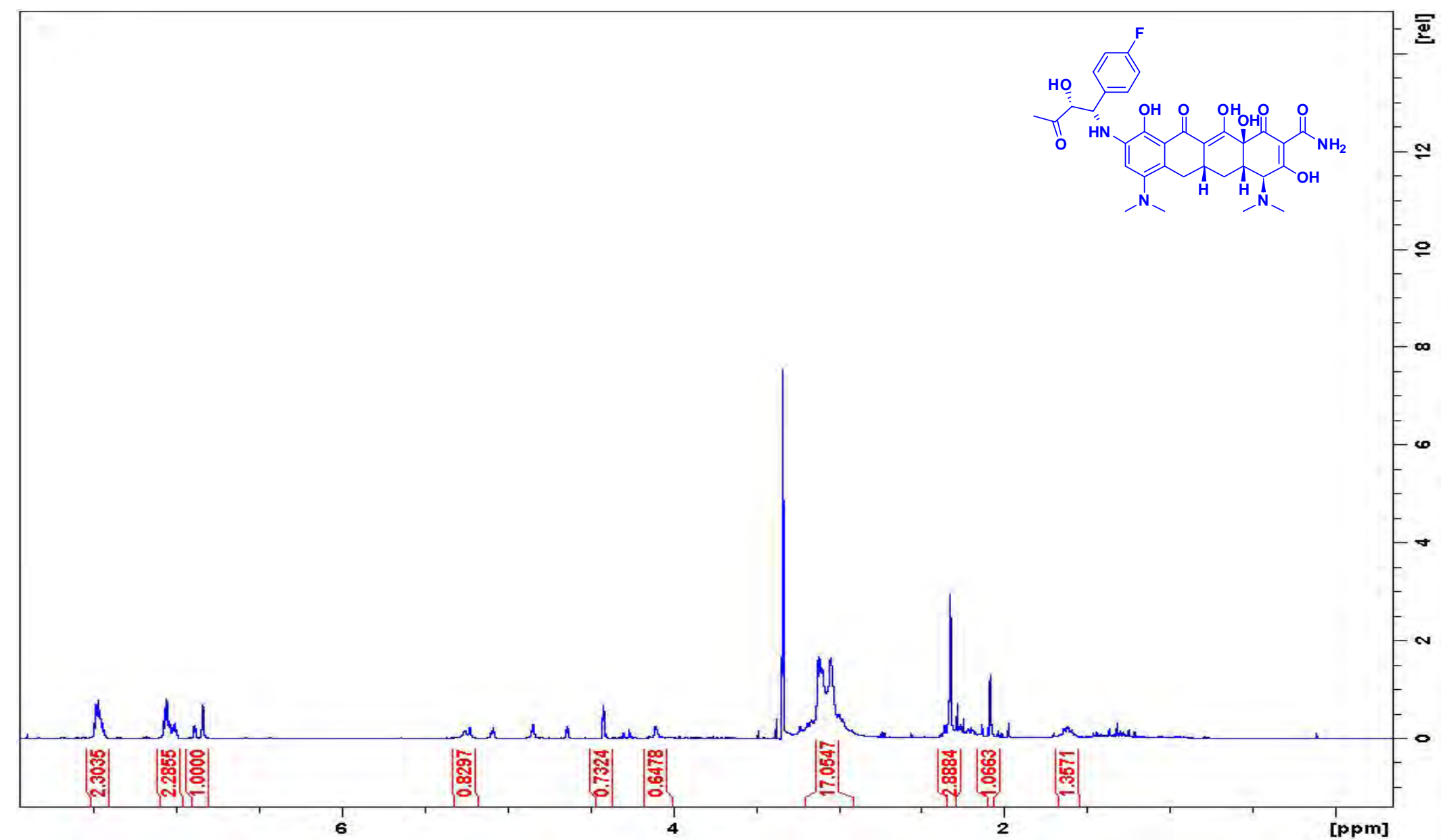


${ }^{1}$ H NMR of (4S,4aS,5aR,12aS)-4,7-bis(dimethylamino)-3,10,12,12a-tetrahydroxy-9-((1S,2R)-2-hydroxy-1-(naphthalen-2-yl)-3oxobutylamino)-1,11-dioxo-1,4,4a,5,5a,6,11,12a-octahydrotetracene-2-carboxamide (2e)

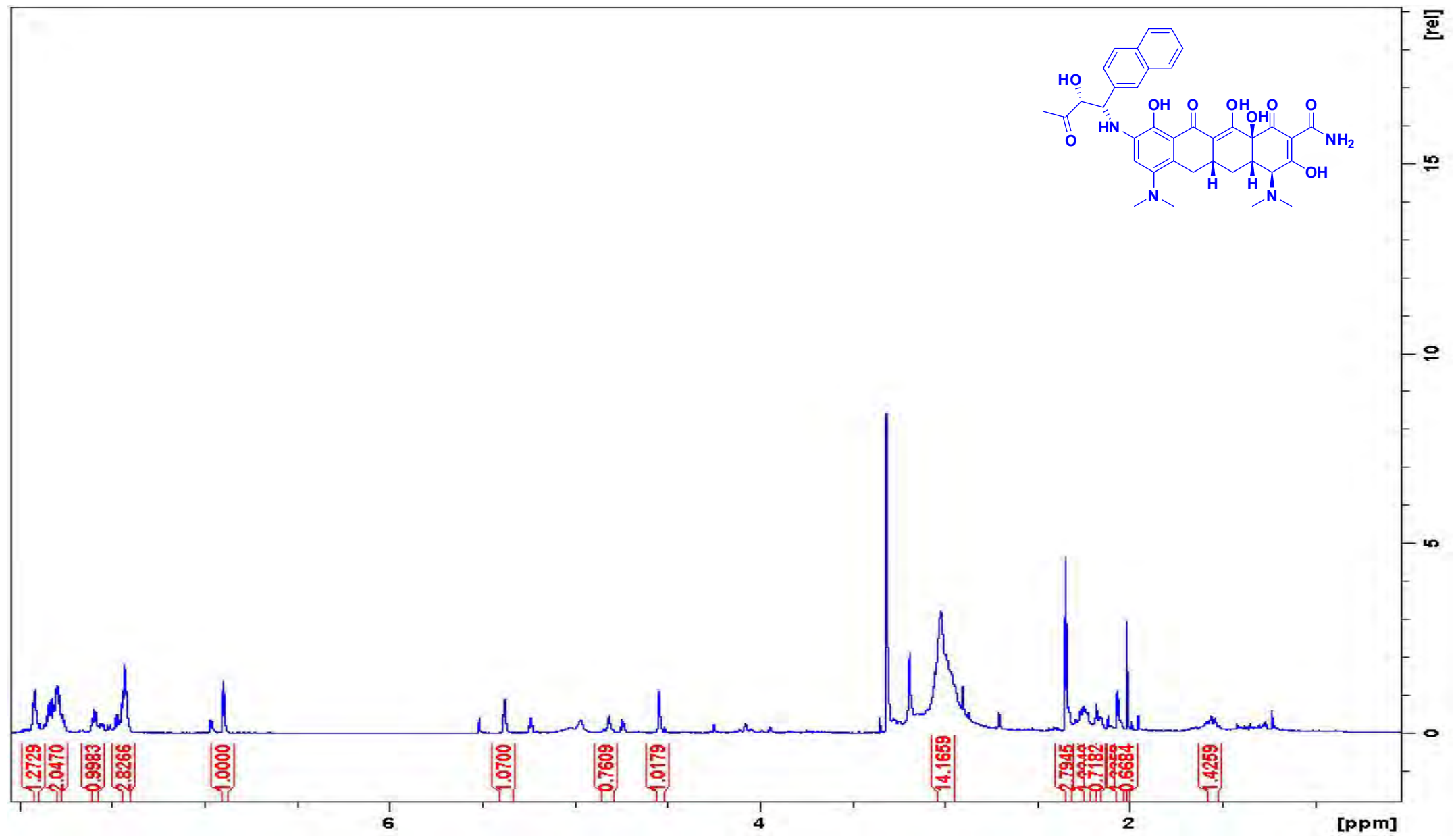


${ }^{1} \mathrm{H}$ NMR of (4S,4aS,5aR,12aS)-4,7-bis(dimethylamino)-3,10,12,12a-tetrahydroxy-9-((1R,2R)-2-hydroxy-1-(5-nitrofuran-2-yl)3-oxobutylamino)-1,11-dioxo-1,4,4a,5,5a,6,11,12a-octahydrotetracene-2-carboxamide (2f)

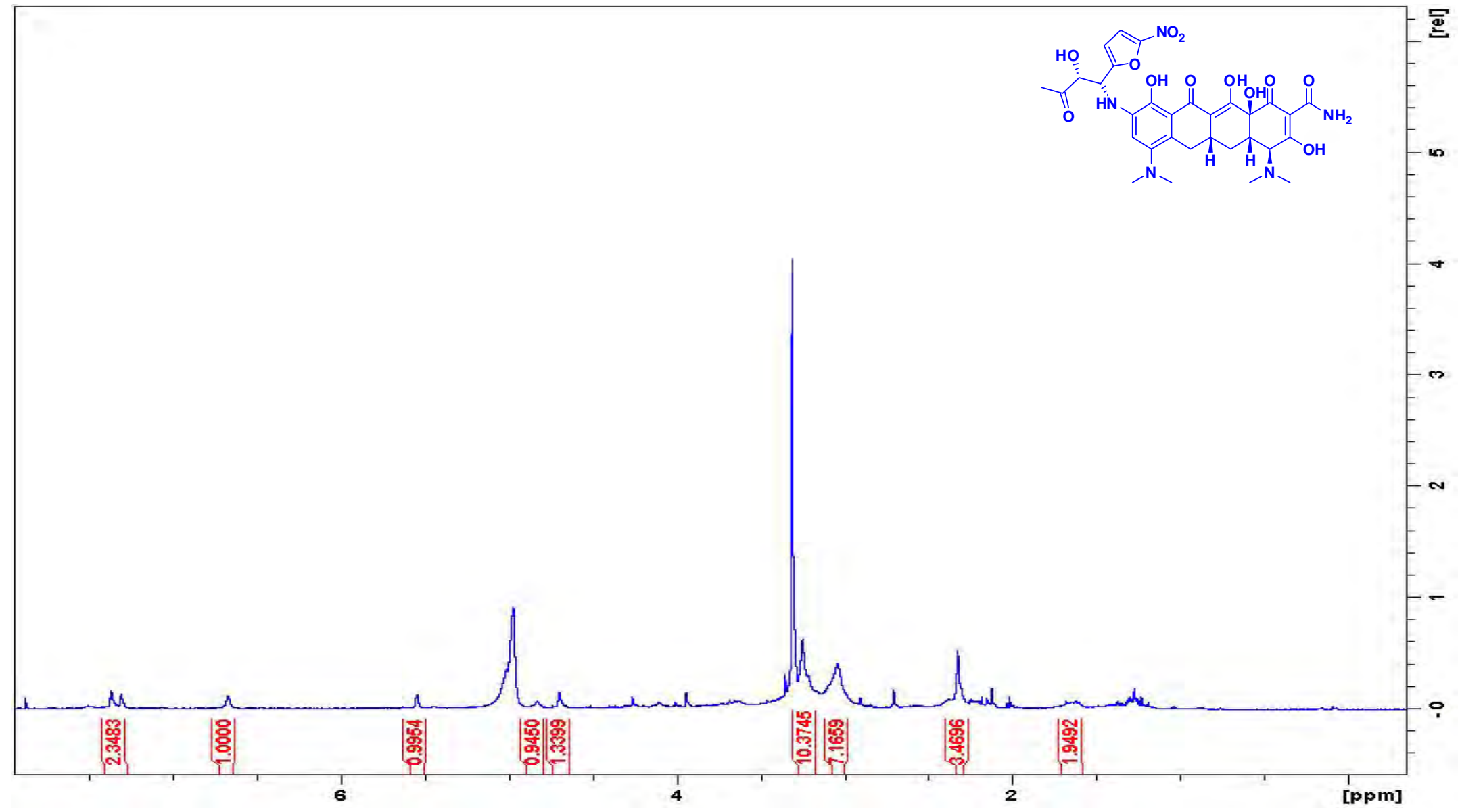


${ }^{1} \mathrm{H}$ NMR of (4S,4aS,5aR,12aS)-4,7-bis(dimethylamino)-9-((1R,2R)-1-(furan-2-yl)-2-hydroxy-3-oxobutylamino)-3,10,12,12atetrahydroxy-1,11-dioxo-1,4,4a,5,5a,6,11,12a-octahydrotetracene-2-carboxamide (2g)

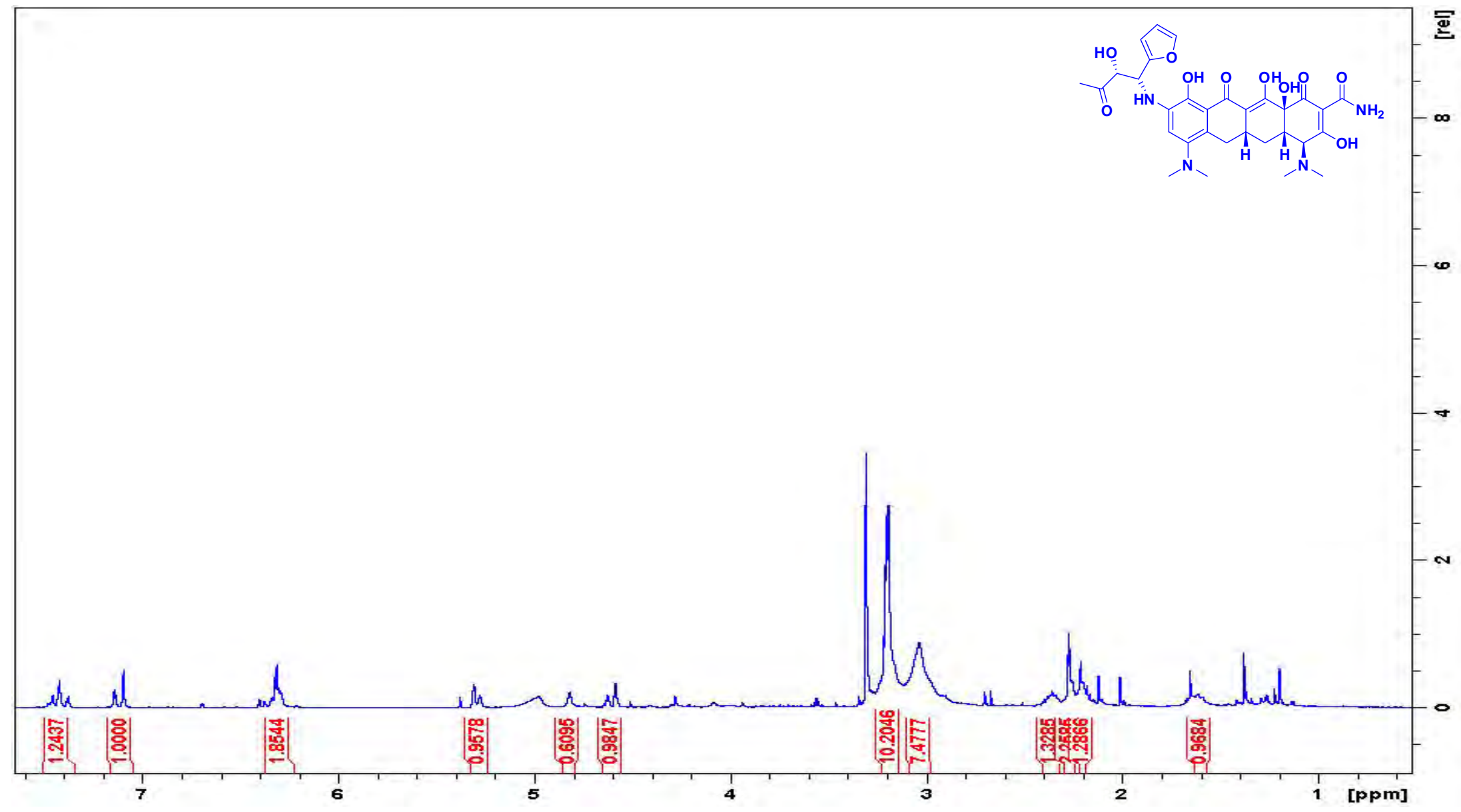


${ }^{1} \mathrm{H}$ NMR of (4S,4aS,5aR,12aS)-9-(1-(5-bromofuran-2-yl)-2-hydroxy-3-oxobutylamino)-4,7-bis (dimethylamino)-3,10,12,12atetrahydroxy-1,11-dioxo-1,4,4a,5,5a,6,11,12a-octahydrotetracene-2-carboxamide (2h)

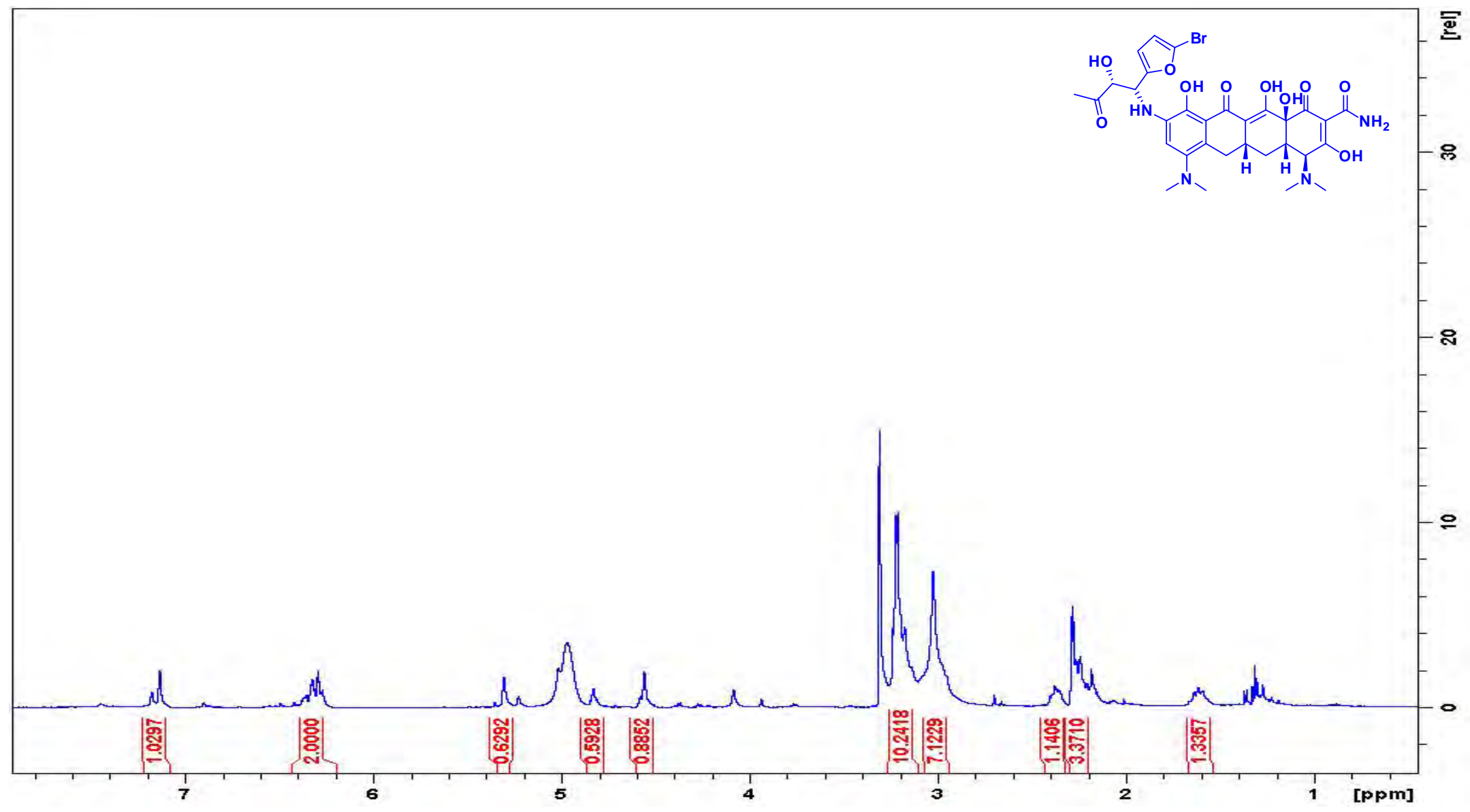

